\title{
Child and Parent Contributions to Maternal Emotion Socialization and Children's Emotion Outcomes in Late Childhood
}

Katie E. Rasmussen

Follow this and additional works at: https://researchrepository.wvu.edu/etd

\section{Recommended Citation}

Rasmussen, Katie E., "Child and Parent Contributions to Maternal Emotion Socialization and Children's Emotion Outcomes in Late Childhood" (2017). Graduate Theses, Dissertations, and Problem Reports. 6480.

https://researchrepository.wvu.edu/etd/6480

This Dissertation is protected by copyright and/or related rights. It has been brought to you by the The Research Repository @ WVU with permission from the rights-holder(s). You are free to use this Dissertation in any way that is permitted by the copyright and related rights legislation that applies to your use. For other uses you must obtain permission from the rights-holder(s) directly, unless additional rights are indicated by a Creative Commons license in the record and/ or on the work itself. This Dissertation has been accepted for inclusion in WVU Graduate Theses, Dissertations, and Problem Reports collection by an authorized administrator of The Research Repository @ WVU.

For more information, please contact researchrepository@mail.wvu.edu. 
Child and Parent Contributions to Maternal Emotion Socialization and Children's Emotion Outcomes in Late Childhood

Katie E. Rasmussen

Dissertation submitted to the

College of Education and Human Services

at West Virginia University

in partial fulfillment of the requirements for the degree of

\section{Doctor of Philosophy}

in

Education

with an emphasis in Human Development and Family Studies

Amy Root, Ph.D., Chair

Paul Hastings, Ph.D.

Reagan Curtis, Ph.D.

Samuel Stack, Ph.D.

Department of Learning Sciences and Human Development

\section{Morgantown, West Virginia}

2017

Keywords: emotion socialization, late childhood, temperament, parenting

(C) 2017 Katie E. Rasmussen 


\title{
ABSTRACT \\ Child and Parent Contributions to Maternal Emotion Socialization and Children's Emotion Outcomes in Late Childhood
}

\begin{abstract}
Katie E. Rasmussen
Parents have an enduring influence on the development of children. The parent-child relationship is dynamic, and both children and their parents contribute to it. The purpose of this investigation was to examine parent-child emotion socialization processes. These processes represent one of the most influential settings where children's emotional trajectories are developed and shaped. Both children and parents shape parental responses to children's negative emotions. In this investigation, both child and parental antecedents were examined simultaneously, including children's negative emotionality, soothability, attention focusing, and inhibitory control, and parental attribution of children's dysregulated specific negative emotions. The final sample included one hundred and twenty-three mother-child dyads and 36 mothers. Mothers reported on their child's temperament and their attributions of children's dysregulated specific negative emotion displays. Children reported on their mother's supportive and nonsupportive emotion socialization responses to specific negative emotions, their emotion regulation strategy use, and regulatory emotional self-efficacy. Standard multiple regression and bootstrapping were used to examine the relations between children's temperament, parental attributions of dysregulated specific negative emotions, emotion socialization responses to specific negative emotions, and children's cognitive reappraisal, expressive suppression, and regulatory emotional self-efficacy. Study findings demonstrated that both children and parents shape parental supportive and nonsupportive responses to specific negative emotions and children's emotion-related outcomes during late childhood.
\end{abstract}




\section{Table of Contents}

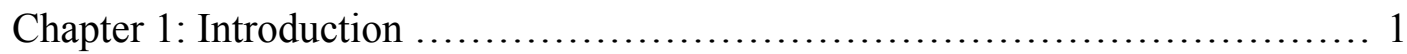

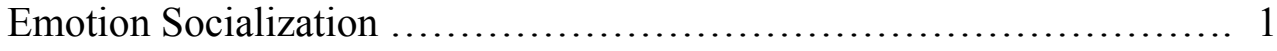

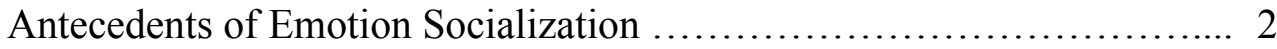

Outcomes of Emotion Socialization $\ldots \ldots \ldots \ldots \ldots \ldots \ldots \ldots \ldots \ldots \ldots \ldots \ldots \ldots$

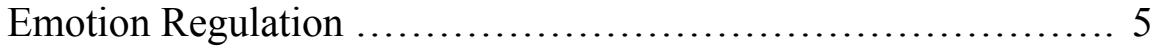

Regulatory Emotional Self-Efficacy ....................... 7

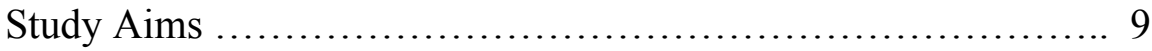

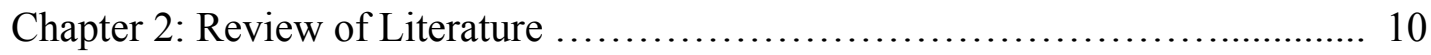

Transactional Theory and the Parent-Child Relationship ............... 11

Child and Parent Contributions to Socialization ................ 12

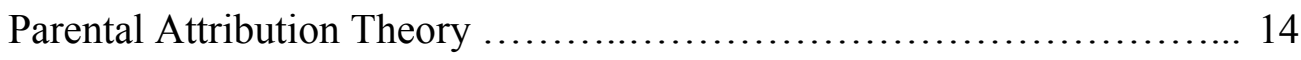

Tripartite Model of the Impact of the Family on Children's Emotion

Regulation and Adjustment ................................ 17

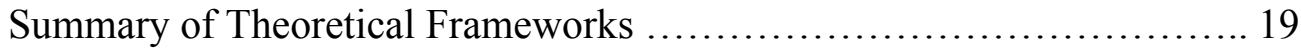

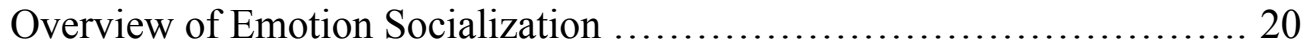

Operationalization of Emotion Socialization ....................... 21

Child Effects on Socialization, Parenting, and Emotion Socialization ..... 24

Parenting Effects on Socialization, Parenting, and Emotion Socialization:

Attributions............................................... 27

Parental Attributions in Late Childhood ............................... 30

Emotion Socialization and Children's Emotion Outcomes ............. 30

Regulatory Emotional Self-Efficacy .......................... 31 
Significance of Proposed Study .................................... 33

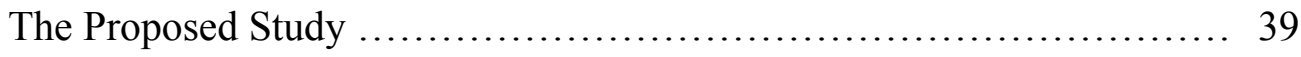

Hypotheses and Research Questions .............................. 40

Research Question One: Child Temperament Predicting Maternal

Attributions of Specific Emotion Displays ................. 41

Research Question Two: Indirect Effects of Child Temperament on

Maternal Emotion Socialization of Specific Emotions via

Maternal Attributions of Specific Emotions................ 41

Research Question Three: Maternal Emotion Socialization as

Moderator Between Child Temperament and Emotion

Outcomes.......................................... 42

Chapter 3: Method …...................................................... 45

Participants and Procedure ............................................ 45

Mother-Report Measures .......................................... 48

Demographic Questionnaire ................................. 48

Emotion Regulation Checklist .............................. 48

Temperament in Middle Childhood Questionnaire ................ 48

Maternal Attributions of Negative Emotion Displays .............. 49

Child-Report Measures ........................................... 50

Emotion Regulation Questionnaire ........................... 50

Regulatory Emotional Self-Efficacy Scale - Adapted ............ 51

Emotions as a Child Inventory ................................ 52

Data Analysis Plan ............................................... 54 
Research Question One

Diagnostic Analyses and Assessment of Assumptions -

Research Question One ......................... 55

Research Question Two ................................ 55

Research Question Three ................................ 56

Diagnostic Analyses and Assessment of Assumptions -

Research Question Three .......................... 56

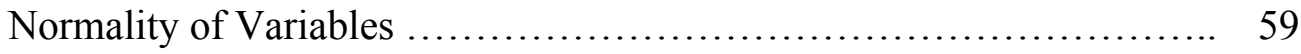

Statistical Power Analyses ..................................... 60

Chapter 4: Results .............................................. 61

Descriptive Statistics and Correlations for Variables of Interest ......... 61

Research Question One: Child Temperament Predicting Maternal

Attributions of Specific Emotion Displays............................. 62

Maternal Attributions of Sadness ......................... 62

Maternal Attributions of Anger $\ldots \ldots \ldots \ldots \ldots \ldots \ldots \ldots \ldots \ldots \ldots \ldots, 62$

Maternal Attributions of Fear .............................. 63

Maternal Attributions of Shame/Embarrassment .............. 63

Research Question Two: Indirect Effects of Child Temperament on

Maternal Emotion Socialization of Specific Emotions via Maternal

Attributions of Specific Emotions $\ldots \ldots \ldots \ldots \ldots \ldots \ldots \ldots \ldots \ldots \ldots \ldots \ldots . \ldots \ldots$

Maternal Attributions of Sadness ........................... 64

Negative Emotionality ........................... 64

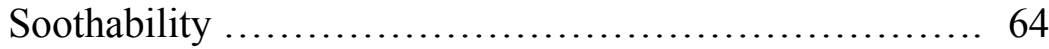




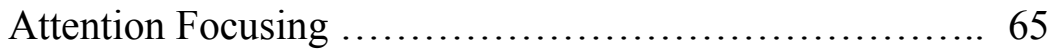

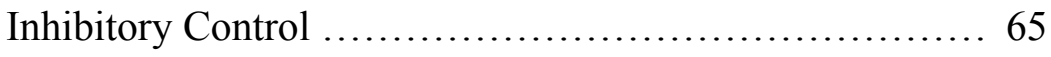

Maternal Attributions of Anger ................................. 65

Negative Emotionality ................................ 65

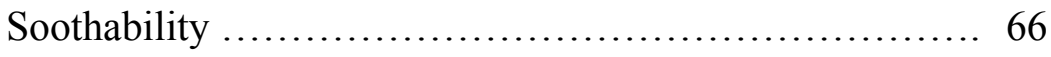

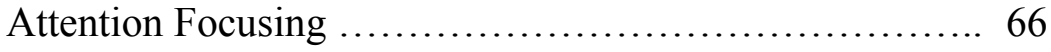

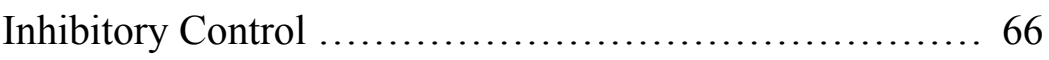

Maternal Attributions of Fear …................................. 66

Negative Emotionality ................................. 66

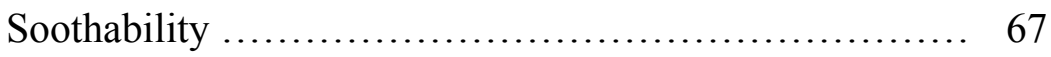

Attention Focusing ................................... 67

Inhibitory Control ..................................... 67

Maternal Attributions of Shame/Embarrassment ............... 67

Negative Emotionality ............................... 67

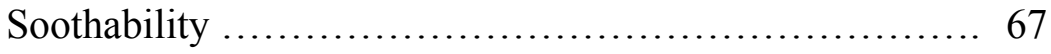

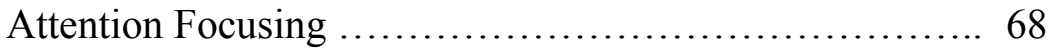

Inhibitory Control .................................... 68

Research Question Three: Maternal Emotion Socialization as Moderator Between Child Temperament and Emotion Outcomes .................. 68

Cognitive Reappraisal....................................... 68

Sadness .......................................... 68

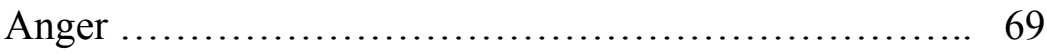

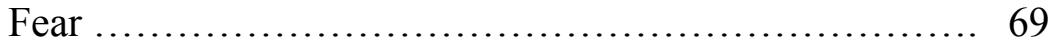


Shame/Embarrassment ................................. 69

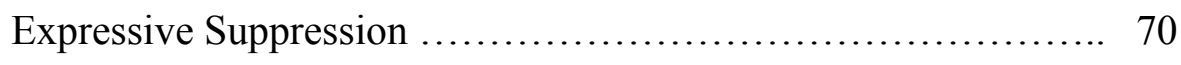

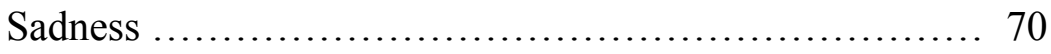

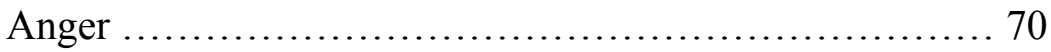

Fear .................................................. 71

Shame/Embarrassment .............................. 71

Regulatory Emotional Self-Efficacy .......................... 72

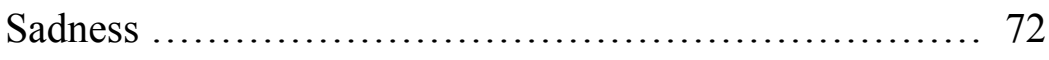

Anger ............................................... 72

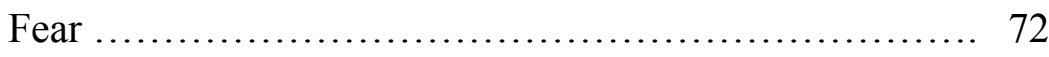

Shame/Embarrassment ................................. 72

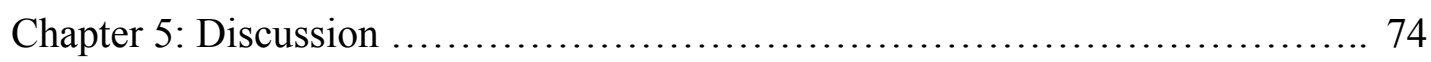

Research Question One: Child Temperament Predicting Maternal

Attributions of Specific Emotion Displays............................... 74

Maternal Attributions of Sadness ............................ 76

Maternal Attributions of Anger ............................. 76

Maternal Attributions of Fear and Shame/Embarrassment .......... 79

Patterns Across Emotions - Predicting Maternal Attributions ..... 80

Research Question Two: Indirect Effects of Child Temperament on

Maternal Emotion Socialization of Specific Emotions via Maternal

Attributions of Specific Emotions .................................. 82

Maternal Attributions of Sadness .............................. 82

Maternal Attributions of Anger ................................ 83 
Maternal Attributions of Fear ................................... 85

Maternal Attributions of Shame/Embarrassment................. 85

Patterns Across Emotions - Indirect Effects of Child

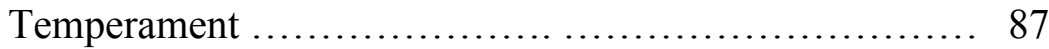

Supportive Versus Nonsupportive Emotion Socialization

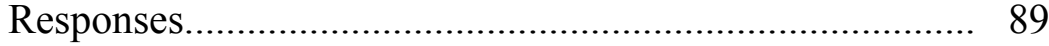

Research Question Three: Maternal Emotion Socialization as Moderator

Between Child Temperament and Emotion Outcomes .................... 91

Cognitive Reappraisal.......................................... 91

Patterns Across Emotions - Cognitive Reappraisal......... 92

Expressive Suppression ....................................... 95

Patterns Across Emotions - Expressive Suppression...... 95

Regulatory Emotional Self-Efficacy .......................... 97

Sadness and Fear ........................................ 97

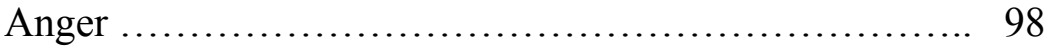

Shame/Embarrassment .............................. 98

Limitations and Future Directions ................................... 99

Applied Implications and Conclusions ….......................... 105

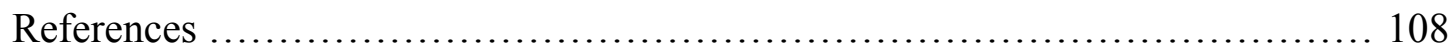




\section{List of Tables}

Table 1 - Diagnostic Criteria and Assumptions for Regression Models for Research Question 1:

Maternal Attributions of Specific Emotions

Table 2 - Diagnostic Criteria and Assumptions for Regression Models for

Research Question 3: Cognitive Reappraisal

Table 3 - Diagnostic Criteria and Assumptions for Regression Models for

Research Question 3: Expressive Suppression

Table 4 - Diagnostic Criteria and Assumptions for Regression Models for

Research Question 3: Regulatory Emotional Self-Efficacy....

Table 5 - Descriptive Statistics for Study Variables of Interest

Table 6 - Pearson Correlations Between Study Variables of Interest 140

Table 7 - Rotated Factor Loadings for Emotion Socialization of Sadness, Anger,

Fear, and Shame/Embarrassment Items

Table 8 - Research Question 1 - Demographic Predictors of Maternal Attributions

of Specific Emotion Displays .................................... 146

Table 9 - Research Question 1 - Predictors of Maternal Attributions of Specific

Emotion Displays

Table 10 - Research Question 2 - Demographic Predictors of Maternal

Nonsupportive Emotion Socialization in Relation to Specific Negative

Emotions 148

Table 11 - Research Question 2 - Demographic Predictors of Maternal Supportive

Emotion Socialization in Relation to Specific Negative Emotions 
Table 12 - Research Question 2 - Indirect Effects of Maternal Attributions of Specific Negative Emotions on the Relation Between Child Temperament

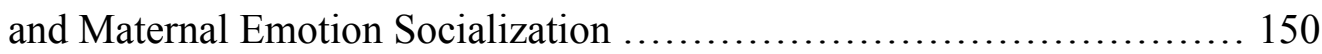

Table 13 - Research Question 2 - Predictors of Maternal Attributions of Sadness and Nonsupportive Emotion Socialization Responses to Sadness ....

Table 14 - Research Question 2 - Predictors of Maternal Attributions of Sadness and Supportive Emotion Socialization Responses to Sadness

Table 15 - Research Question 2 - Predictors of Maternal Attributions of Anger and Nonsupportive Emotion Socialization Responses to Anger

Table 16 - Research Question 2 - Predictors of Maternal Attributions of Anger and Supportive Emotion Socialization Responses to Anger

Table 17 - Research Question 2 - Predictors of Maternal Attributions of Fear and Nonsupportive Emotion Socialization Responses to Fear

Table 18 - Research Question 2 - Predictors of Maternal Attributions of Fear and Supportive Emotion Socialization Responses to Fear 158

Table 19 - Research Question 2 - Predictors of Maternal Attributions of Shame/ Embarrassment and Nonsupportive Emotion Socialization Responses to Shame/Embarrassment

Table 20 - Research Question 2 - Predictors of Maternal Attributions of Shame/ Embarrassment and Supportive Emotion Socialization Responses to Shame/Embarrassment

Table 21 - Research Question 3 - Demographic Predictors of Child Cognitive Reappraisal, Expressive Suppression, and Regulatory Emotional 
Self-Efficacy of Negative Emotions ....................................................... 161

Table 22 - Research Question 3 - Predictors of Child Cognitive Reappraisal...... 162

Table 23 - Research Question 3 - Predictors of Child Expressive Suppression ... 164

Table 24 - Research Question 3 - Predictors of Child Regulatory Emotional

Self-Efficacy of Negative Emotions ................................. 166 


\section{List of Figures}

Figure 1 - Conceptual Model for Research Question Two: Indirect Effects

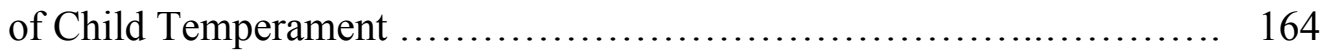

Figure 2 - Conceptual Model for Research Question Three: Moderation ......... 169

Figure 3 - Research Question 3 - Maternal Nonsupportive Emotion Socialization in Response to Shame as Moderator Between Child Negative Emotionality and Regulatory Emotional Self-Efficacy of Negative Emotions ........... 170

Figure 4 - Research Question One: Summary of Significant Findings ............ 171

Figure 5 - Research Question Two: Summary of Significant Findings ........... 172

Figure 6 - Research Question Three: Summary of Significant Findings ............ 174 


\section{List of Appendices}

Appendix 1 - Demographic Questionnaire .................................. 176

Appendix 2 - Emotion Regulation Checklist ................................. 182

Appendix 3 - Maternal Attributions of Negative Emotion Expressions .......... 185

Appendix 4 - Emotion Regulation Questionnaire (Child Version) ................ 189

Appendix 5 - Regulatory Emotional Self-Efficacy Scale - Adapted ............. 192

Appendix 6 - Emotions as a Child Inventory (Child-Report) …................. 195

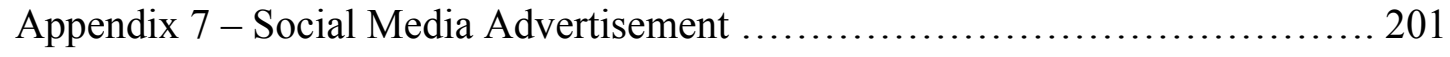

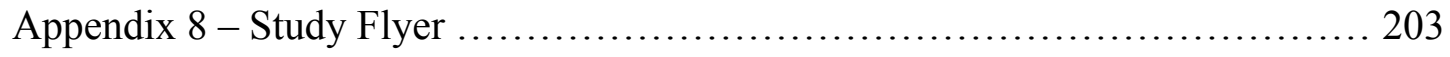

Appendix 9 - Electronic Cover Letter ...................................... 205 


\section{Chapter 1}

\section{Introduction}

Parents have an enduring influence on the development of children (Grusec \& Davidov, 2015). The parent-child relationship is dynamic, and both children and their parents contribute to it (Belsky, 1984; Grusec \& Davidov, 2015; Sameroff, 1975). There are many different domains of the parent-child relationship, and the purpose of this investigation was to examine one specific domain, parent-child emotion socialization processes. Parental emotion socialization is one of the most influential processes that shape children's emotional trajectories (Sheffield Morris, Silk, Steinberg, Myers, \& Robinson, 2007). As a result, emotion socialization processes are of critical importance to study (Eisenberg, Cumberland, \& Spinrad, 1998).

Emotion socialization is conceptualized in several different ways. Specifically, it includes parents' philosophies about the acceptability and potential harm of emotions and emotion expression (Gottman, Katz, \& Hooven, 1996) and parents' reactions to children's emotions (Fabes, Poulin, Eisenberg, \& Madden-Derdich, 2002; O’Neal \& Magai, 2005). In studies examining the reactions to children's emotions, there are two major categories of reactions, supportive and nonsupportive reactions. Supportive emotion socialization behaviors encourage children's expression of emotions and help children to manage their emotional experiences (Blair et al., 2014; Spinrad et al., 2007; Taylor, Eisenberg, Spinrad, Eggum, \& Sulik, 2013; Warren \& Stifter, 2008). Nonsupportive reactions to emotions punish children's emotional expressions (Buckholdt, Parra, \& Jobe-Shields, 2014; Eisenberg et al., 1999; Garside \& Klimes-Dougan, 2002; Luebbe, Kiel, \& Buss, 2011; Shewark \& Blandon, 2015). 


\section{Antecedents of Emotion Socialization}

Emotion socialization processes are influenced by both child- and parent-level factors (Buckholdt et al., 2014; Eisenberg \& Fabes, 1994). As a result, this study examined the contribution of both parents and children and examined two antecedents of emotion socialization responses, namely, children's temperament and maternal attributions of children's dysregulated negative emotion expressions. Children contribute to emotion socialization processes through the dispositional characteristics they contribute to the parent-child relationship (Belsky, 1984;

Eisenberg \& Fabes, 1994). In early life, children's emotional experiences are profoundly shaped by their temperamental traits that either promote or undermine emotion regulation, and these traits shape the way parents respond to children's emotions (Belsky, 1984; Calkins \& Dollar, 2014; Shields \& Cicchetti, 1995) For the purposes of this study, four temperamental traits related to children's emotionality and regulatory behaviors were examined, including negative emotionality, soothability, attention focusing, and inhibitory control. Negative emotionality is defined as dispositional proneness to experiencing high levels of negative emotions (e.g., sadness, anger, fear) that makes emotion regulation more difficult (Rothbart \& Bates, 2006). On the other hand, soothability (how well the child can recover from emotional arousal), attention focusing (the ability to attend to important stimuli relevant to an emotional situation), and inhibitory control (the ability to stop a dominant, less adaptive response in an effort to enact a more effective, adaptive one) are temperamental traits that help promote emotion regulation (Rothbart \& Bates, 2006).

In addition, children who are high in negative emotionality and have difficulty regulating their emotions elicit harsher forms of parenting than children who do not have difficulty (Laukkanen, Ojansuu, Tolvanen, Alatupa, \& Aunola, 2014). However, it is likely that 
temperament affects parenting practices indirectly via parental attributions. The attributions parents make about children's behaviors influence parents' responses to children (Slep \& O’Leary, 1998). Parental attributions are a specific form of cognition and are internal knowledge structures that guide parents' behavior in caregiving scenarios (Bugental \& Happaney, 2002; Dix, Ruble, \& Zambarano, 1989; Slep \& O’Leary, 1998).

Indeed, studies demonstrate that parents who ascribe internal attributions for children's behavior (i.e., children's behaviors are dispositional, intentional, are consistent, and under the child's control) are harsher and more nonsupportive in interactions with their children (Coplan, Hastings, Lagacé-Séguin, \& Moulton, 2002; Slep \& O’Leary, 1998). On the other hand, external attributions describe parental cognitions that attribute children's behavior being due to a temporary state, the behavior was unintentional, the behavior is not likely to continue, and the behavior was not under the child's control (Coplan et al., 2002). Mothers who endorse more external attributions for children's misbehaviors use more inductive discipline and are less likely to use punishment than mothers who endorse internal attributions of these behaviors (Dix et al., 1989).

Thus, when parents perceive that children's emotionally dysregulated behavior (e.g., difficulty calming down when angry) is caused by temperament, they may respond to their children's emotions in a nonsupportive way. However, this hypothesis has not been empirically tested; thus, it remains unknown why some caregivers respond to their children's emotions in a nonsupportive fashion. Given that nonsupportive emotion socialization strategies are associated with a variety of negative child outcomes, it is important to further understand antecedents of these potentially harmful parenting responses (e.g., internalizing and externalizing difficulties, Buckholdt et al., 2014; poorer emotion awareness, Warren \& Stifter, 2008; expressive 
suppression (Gunzenhauser, Fäsche, Friedlmeier, \& von Suchodoletz, 2014). Thus, the first aim of the proposed study was to examine children's temperament (i.e., negative emotionality, soothability, attention focusing, and inhibitory control) as a predictor of maternal attributions of specific negative emotion displays.

The majority of evidence linking parental attributions with parents' behavior is in the general parenting literature. However, it is likely these linkages exist within parent-child emotion socialization processes. Emotion socialization processes are a sub-domain of the parent-child relationship and is nested within the larger parent-child context (Chan, Bowes, \& Wyver, 2009; Sheffield Morris et al., 2007). It is likely that attributional patterns at play within the general parent-child relationship may trickle down to emotion socialization processes (Chan et al., 2009; Sheffield Morris et al., 2007). As noted above, parents' attributions inform their responses to their children and are influenced by children's temperament (Bugental, 1987; Bugental \& Happaney, 2002; Dix, 1993; Slep \& O’Leary, 1998). When parents draw internal attributions for a particular behavior (e.g., the behavior is dispositional, the child intentionally behaved in a certain way; Coplan et al., 2002), they respond in more negative ways (Dix et al., 1989; Slep \& O’Leary, 1998). When parents draw external attributions for a behavior, they tend to respond less negatively, such as using an inductive discipline approach rather than simply punishing the child (Dix et al., 1989).

In the emotion socialization literature, it is well-documented that children's temperament influences mothers' and fathers' responses to children's emotions (Eisenberg \& Fabes, 1994). It is also likely, but has not been studied, that parents' internal attributions also may translate to more nonsupportive emotion socialization behaviors, such as neglectful parental responses to or punishment of children's expressions of negative emotions; external attributions for child 
behavior may translate to more supportive parental emotion socialization behaviors, such as comforting the child or trying to distract them from the emotion they are expressing. Thus, it is likely that the reason that previous work has indicated a link between children's temperament and parents' responses to children's negative emotions is from parents' attributions (i.e., attributions indirectly affect the relation between temperament and emotion socialization). Therefore, the second aim of this study was to examine maternal attributions regarding children's expressions of negative emotion as an indirect effect on the association between children's emotionality and regulatory behaviors and maternal emotion socialization responses.

\section{Outcomes of Emotion Socialization}

Parental emotion socialization behaviors profoundly influence different aspects of children's emotional functioning. Three outcomes related to children's emotional functioning that will be examined in this study are children's use of cognitive reappraisal, expressive suppression, and their regulatory emotional self-efficacy. Emotion regulation is defined as the "processes used to manage and change if, when, and how (i.e., how intensely) one experiences emotions and emotion-related motivational and physiological states, as well as how emotions are expressed behaviorally" (Eisenberg, Hofer, \& Vaughan, 2007, p. 288). In infancy and early childhood, emotion regulation processes are primarily directed by others through caregivers' soothing behaviors that help children calm themselves (Calkins \& Dollar, 2014). As children develop and enter later childhood and pre-adolescence, a shift takes place and emotion regulation becomes more effortful, cognitively-driven, and intentional. This change allows children to purposefully engage in regulatory strategies such as cognitive reappraisal and expressive suppression (Rawana, Flett, McPhie, Nguyen, \& Norwood, 2014). 
Importantly, emotion regulatory strategies are likely influenced by dispositional emotionality, however, the two are distinct constructs. Indeed, differing measures of emotion regulation focus on one element of emotion regulation over another, such as primarily temperamentally-based instruments versus measures that focus on more intentional, cognitive aspects of emotion regulation (e.g., temperamentally-based, Shields \& Cicchetti, 1995; intentional, cognitive aspects of emotion regulation, Gullone \& Taffe, 2012). As children enter late childhood and transition to adolescence, emotion regulation becomes much more selfdirected, purposeful, intentional, and more complex than in earlier childhood (Rawana et al., 2014). During this period, children have a more complex understanding of the meaning of emotions and are able to exercise more complex regulatory strategies that are adaptive for a particular regulatory situations at-hand (Labouvie-Vief, DeVoe, \& Bulka, 1989; ZimmerGembeck \& Skinner, 2011). Cognitive reappraisal and expressive suppression in particular also reflect children's psychological adjustment during this developmental period (Riedeger \& Klipker, 2014; Betts, Gullone, \& Allen, 2009)

For the purposes of this study, temperamentally-based negative emotionality will be differentiated from use of emotion regulation strategies that children purposefully engage in. In this investigation, negative emotionality will be examined as an antecedent of emotion socialization, while emotion regulation strategy use (i.e., cognitive reappraisal and expressive suppression) will be examined as an outcome of emotion socialization to reflect children's developing regulatory abilities during this age period. Taken together, the examination of emotion regulation strategies (i.e., cognitive reappraisal and expressive suppression) is particularly important in a late childhood sample, and provides unique information regarding children's use of purposeful emotion regulation strategies. 
As aforementioned, emotion regulatory strategies are influenced by temperament, but it is also important to note that they also are influenced by socialization processes (Eisenberg \& Fabes, 1994; Eisenberg \& Valiente, 2004; Rothbart, Sheese, \& Posner, 2014). For instance, Root, Byrne, and Watson (2015) reported that temperamentally inhibited children engaged in more adaptive emotion regulatory strategies during a fear task when their mothers engaged in higher levels of supportive reactions to fear. In addition, Liew and colleagues (2003) found that boys who displayed higher levels of physiological reactivity were more emotionally wellregulated when their mothers displayed low levels of negative emotion expressivity. Taken together, these studies suggest that emotion socialization processes may moderate the relation between other temperamental traits and children's emotion-related outcomes.

Another component of children's emotional functioning that may be affected by temperament and emotion socialization behaviors is children's regulatory emotional selfefficacy. Self-efficacy is defined as an individual's sense of control over certain aspects of their behavior and events in their environment (Bandura, 1997). An individual's sense of self-efficacy to engage in any behavior or skill is critical to consider when examining the development of certain behaviors. This happens because self-efficacy underlies an individual's motivation to engage in certain situations and it shapes the amount of effort an individual expends to achieve a goal or engage in a behavior (Bandura, 2001).

In addition, children's regulatory emotional self-efficacy is a reflection of cognitive gains achieved and developments in complexity in emotion regulation during late childhood (e.g., metacognition; Miller, Hardin, \& Montgomery, 2003; Rawana et al., 2014). As a result, the examination of regulatory emotional self-efficacy taps into older children's more complex understanding of themselves and their regulatory responses and patterns (Miller et al., 2003; 
Riedeger \& Klipker, 2014). Regulatory emotional self-efficacy is also beneficial for children's developmental outcomes, and contributes to other domains of self-efficacy, such as academic self-efficacy, and decreased levels of internalizing and externalizing difficulties (Bandura, Caprara, Barbaranelli, Gerbino, \& Pastorelli, 2003; Caprara, Steca, Gerbino, Paciello, \& Vecchio, 2006). For example, when a child feels able to effectively regulate their emotions, this sense of self-efficacy promotes feelings of competence in social situations (Caprara et al., 2005). It is likely, however it has not been empirically tested, that children's temperamental traits are related to regulatory emotional self-efficacy. Specifically, it is likely that if children are low in negative emotionality and high in soothability, attention focusing, and inhibitory control, they will feel efficacious to regulate their emotions simply because it is easy for them to return to a regulated state following emotional arousal. Parents also likely play a role in supporting or undermining children's regulatory emotional self-efficacy, particularly with children who are higher in negative emotionality or have difficulty regulating their emotions (van der Bruggen, Stams, Bögels, \& Paulussen-Hoogeboom, 2010).

Previous research indicates that maladaptive parenting practices are associated with emotion dysregulation and a poorer sense of general self-efficacy (Buckholdt et al., 2014; Givertz \& Segrin, 2012). Thus, it is likely that the use of nonsupportive emotion socialization responses will negatively influence children's use of cognitive reappraisal and expressive suppression and their regulatory emotional self-efficacy. Emotion socialization responses that punish, dismiss, or ignore children's emotional displays lead to emotion dysregulation that inhibits a child from using effective regulatory strategies (Buckholdt et al., 2014). Nonsupportive parental emotion socialization behaviors may also damage children's sense of self-efficacy to regulate by communicating to a child either verbally (e.g., "stop being such a baby, it's not a big 
deal") or implicitly (e.g., responding with negativity or hostility) that the child is overly emotional or unable to regulate their emotions effectively. Supportive responses may play an equally important role in promoting these outcomes by teaching children when and how to use effective emotion regulation strategies (Sheffield Morris et al., 2007). When children are taught about and practice emotion regulation strategies with the help of parents, they are more likely to feel efficacious in their abilities to regulate their emotions as well. Children who are higher in negative emotionality or lower in soothability, attention focusing, or inhibitory control may be

particularly impacted by supportive or nonsupportive emotion socialization responses (Sheffield Morris, et al., 2007). Thus, the third aim of the proposed study was to examine if maternal emotion socialization responses moderated the relation between child temperament (i.e, negative emotionality, soothability, attention focusing, and inhibitory control) and children's emotionrelated outcomes, namely, cognitive reappraisal, expressive suppression, and regulatory emotional self-efficacy.

\section{Study Aims}

In sum, there were three aims of this study. The first aim was to examine how children's negative emotionality, soothability, attention focusing, and inhibitory control predicted maternal attributions of children's expressions of dysregulated negative emotions. The second aim was to investigate whether children's temperament indirectly affected maternal supportive and nonsupportive responses to specific emotions via maternal attributions of each emotion.

Finally, the third aim of this study was to examine whether maternal emotion socialization responses moderated the relation between children's negative emotionality, soothability, attention focusing, and inhibitory control and their cognitive reappraisal, expressive suppression, and regulatory emotional self-efficacy. 


\section{Chapter 2}

\section{Review of Literature}

Over time, developmental scientists have established the dynamic nature of the parentchild relationship (Kuczynski \& Parkin, 2007; Maccoby, 2015). Children and parents influence one another in ongoing parent-child interactions, and these interactions form the way parents think about children's behaviors and choose to respond to children (Belsky, 1984; Bugental \& Happaney, 2002; Kuczynski \& Parkin, 2007; Sameroff, 1975). Using concepts of transactional theory, the parent-child relationship will be described and discussed in relation to socialization processes. In addition, children and parents' contribution to socialization processes will be discussed, including the child effects literature. Finally, parental attribution theory will be discussed to frame how parental attributions are made. It is important to note that this study examined only two factors that shape parent-child socialization interactions, parents, and children. Contemporary perspectives of socialization posit that the transactional, back-and-forth socialization processes described below are complex and do not solely happen between parents and children (Smetana, Robinson, \& Rote, 2015). Indeed, many additional transactional socialization processes and socialization agents beyond parents and children influence children's trajectories. For example, peer relationships (Criss, Houltberg, Cui, Bosler, Sheffield Morris, \& Silk, 2016), mass media (Uhls \& Greenfield, 2012; O'Keeffe \& Clarke-Pearson, 2011; Prot et al., 2015), culture (Cole \& Tan, 2015), and social class (Wilkins \& Pace, 2014) are all critical socialization agents in shaping children's trajectories. Taken together, while acknowledging this clear complexity in socialization processes and agents, the theoretical work discussed below will focus on children and parents as socialization agents. 


\section{Transactional Theory and the Parent-Child Relationship}

Children's socialization experiences are rooted in human relationships, particularly the parent-child relationship (Kuczynski \& Parkin, 2007). External to children, parents have the most enduring impact on children's development (Grusec \& Davidov, 2015). However, children influence the parent-child relationship and how parents interact with them (Belsky, 1984; Grusec \& Davidov, 2015; Sameroff, 1975). Bidirectional, transactional approaches to socialization posit that in the parent-child relationship, both parents and children can initiate intentional behavior and choose behaviors that will influence one another (Kuczynski \& Parkin, 2007). These abilities represent the capacity to reflect on one's behavior and interpret the messages exchanged between parent and child during ongoing interactions (Kuczynski \& Parkin, 2007). As a result, both parents and children are agentic in their own right. As socialization agents, parents and children engage in purposeful behaviors at some times, while at other times, socialization behaviors may be automatic and unconscious (Kuczynski \& Parkin, 2007; Kucznyski, Harach, \& Bernardini, 1999). Over time, these interactions transform both members of the dyad as each responds to the behavior of the other (Kuczynski \& Parkin, 2007; Sameroff, 1975). In terms of children's outcomes specifically, children's trajectories are best understood within the transactional, dynamic context of child and parent behavior, rather than as a function of child or parent alone (Sameroff, 1975).

While the proposed study was not longitudinal and cannot test for true transactional parent-child effects, these principles of transactional theory provide important conceptual context for the antecedents of maternal emotion socialization examined in this study, specifically, components of children's temperament (i.e., negative emotionality, soothability, attention focusing, and inhibitory control) and maternal attributions of specific negative emotion displays. 
For example, the transactional approach emphasizes parental behaviors are the product of the influence of both children and parents. Conceptually, this theory also helps describe the processes by which children influence parental behaviors. Parents observe children's behaviors over the course of ongoing interaction, make meaning of what children do, and use this information when choosing how to respond (Kuczynski \& Parkin, 2007; Sameroff, 1975). Based on these principles, in this investigation, the assumption is made that children's temperamental traits (i.e., negative emotionality, soothability, attention focusing, inhibitory control) will impact the ways parents think about and explain the way their children express negative emotions. As a result, parental emotion socialization responses are very likely informed by the explanations they have generated for their children's behaviors.

Child and parent contributions to socialization. As noted above, children are active agents in socialization processes and make important contributions to their caregiving experiences (Kuczynski \& Parkin, 2007). While parents' behaviors are shaped by multiple factors, children's characteristics represent one of the three major determinants of parenting described by Belsky (1984). This proposition is reflected in the child effects literature that suggests important ways children shape the quantity and quality of parenting experiences (Bell \& Chapman, 1986; Belsky, 1984). Children's behavior serves as a trigger for parental affect and cognition, activating cognitive processes that shape parental behaviors (Dix, 1991). When parents observe and interact with children, their own thoughts and emotions are activated by what their children do, especially behaviors that may be frustrating or challenging to parents (Dix, 1991). In particular, children who are perceived as difficult elicit or become targets of harsh or disapproving responses from adults (e.g., Bugental, Shennum, \& Shaver, 1984). For example, children's displays of negative emotions are associated with less adaptive parenting 
behaviors (Lipscomb, Harold, Shaw, Leve, Neiderhiser, \& Ge, 2011; Paulussen-Hoogeboom, Stams, Hermanns, Peetsma, 2008; van der Bruggen et al., 2010). Indeed, high levels of negative emotionality are associated with maternal controlling parenting in early childhood (Laukkanen et al., 2014; Paulussen-Hoogeboom et al., 2008; van der Bruggen et al., 2010). Thus, children themselves shape the nature and type of parental socialization behaviors parents engage in during transactional parent-child interactions (Kuczynski \& Parkin, 2007; Mills \& Rubin, 1990; Sameroff, 1975).

It is relatively well-documented that children's dispositional traits affect their parents' practices and behaviors (e.g., Crockenberg \& Smith, 1982; Kennedy, Rubin, Hastings, \& Maisel, 2004); the link between parents' attributions and parenting behaviors has also been empirically tested (e.g., Slep \& O'Leary, 1998). However, these processes have yet to be studied in the within the specific context of emotion socialization processes. This is important because of the pivotal role emotion socialization processes play in either promoting or undermining children's emotional outcomes (Blair et al., 2014; Buckholdt et al., 2014). If parental attributions translate to more or less supportive or nonsupportive emotion socialization responses, specific parental attributions that put parent-child dyads at-risk will be identified.

In addition to the transactional theory of parenting, the proposed study is also guided by parental attribution theory (e.g., Bugental \& Happaney, 2002; Dix \& Grusec, 1985) and the tripartite model of the impact of the family on children's emotion regulation and adjustment (Sheffield Morris et al., 2007). In the section below, an overview of these theories is provided, with a discussion of how they connected to the proposed investigation. 


\section{Parental Attribution Theory}

In addition to children's contributions to the parent-child interactions, parents themselves also make an important contribution to socialization processes. Parents' cognitions about their child and events that take place in the parent-child caregiving relationship represent an important antecedent of parental behavior that help parents make meaning of their experiences and shape their caregiving behavior (Mills \& Rubin, 1990; 1992; Slep \& O’Leary, 1998).

Theoretical work on attributions describes how parental attributions influence parental behavior. Individuals seek to explain events that require a response, or are undesirable, novel, or unclear (Bugental, 1987; Bugental \& Happaney, 2002). In these types of situations, individuals are more apt to generate a causal explanation or attribution for these types of behaviors because it helps them to act adaptively and use their existing knowledge base to decide how to respond than for situations that make more sense (Bugental \& Happaney, 2002; Pratto \& John, 1991). A parent may use information about a similar caregiving situation they have already encountered to help them understand how to respond in a new situation. Thus, parental attributions influence how parents respond to children's behaviors.

Developmental scholars applied the construct of attributions and attribution theory to parents and parenting processes (e.g., Coplan et al., 2002; Dix \& Grusec, 1985). Parental attributions refer to explanations parents make to account for children's behavior or characteristics (Bugental \& Happaney, 2002; Coplan et al., 2002; Mills \& Rubin, 1990). While some parenting behaviors are spontaneous, parents are agentic and meaning-making entities in the parent-child relationship with the ability to interpret and respond to children's ongoing behaviors (Bugental \& Johnston, 2000; Kuczynski \& Parkin, 2007). As a result, parents develop 
attributions about their child's traits (e.g., being particularly emotional or shy) and behavior (e.g., having frequent emotional outbursts) that guide parental responses to children.

In addition, Dix and Grusec (1985) specifically applied attribution theory to parenting processes (Weiner, 1971; Weiner et al., 1985). They created a conceptualization of parental attributions that identified three specific dimensions of parental attributions. These three dimensions include the intentionality of a child's behavior, the degree to which the child can control the specific behavior, and the degree to which the behavior is characteristic of the child's disposition rather than situational constraints. Rubin and Mills (1990; 1992) built upon Dix and Grusec's (1985) work, conceptualizing parental attributions based on locus (i.e., internal or external to the child) and attribution type (i.e., stable or unstable). Others (e.g., Coplan et al., 2002; Hastings \& Rubin, 1999) have taken similar approaches, but examined parental attributions as a single continuous variable. In this conceptualization, parental attributions are assessed on a single continuum based on four dimensions of the behavior, including how stable, intentional, typical, and dispositional the behavior is. Internal attributions are one end of the continuum (i.e., the behavior is stable, intentional, typical of the child, and resulting from the child's disposition), and external attributions are on the other (i.e., the behavior is unstable and a stage that will pass, the child didn't mean to do the behavior, the behavior was not typical of the child, and the behavior was due to the situation; Coplan et al., 2002). This latter conceptualization of internal and external attributions as a single continuous variable will be used in this investigation.

Parental attributions are influenced by both proximal and distal factors. Distally, parents are impacted by their own experiences as children and their internal working model developed from their relationships with their parents (Bugental \& Happaney, 2002). Parental attributions 
are shaped by the parenting behaviors modeled to them their own parents; and, to a degree, parental attributions are parents' early relationship experiences projected outward toward their children (Bugental, 1987; Bugental \& Happaney, 2002). For example, if a parent was taught as a child that $\mathrm{X}$ behavior results from willful child disobedience, they are likely to attribute the behavior in the same way with their own children. Moreover, parents' attributional style for interpreting events in their own lives is related to the way they attribute events in their children's lives (Heatherington, McDonald, Tolejko, \& Funk, 2007). For example, when parents draw more other-blaming and punishing attributions toward events in their own lives, they are more likely to apply these types of attributions toward their children (Heatherington et al., 2007). Furthermore, parents' attributions are affected by contextual factors such as socioeconomic status and support (Mills \& Rubin, 1992). Thus, parental attributions appear to be influenced by present and past experiences and are affected by important contextual influences.

While these distal influences on parental attributions are clearly important, the focus of the proposed investigation is comparatively proximal influences on parents' attributions, particularly children's temperamental traits. Parental attributions are influenced by several proximal factors, such as the presence of their children, and by their children's characteristics and ongoing behavior (Bugental, 1987; Bugental \& Happaney, 2002; Dix, 1993; Mills \& Rubin, 1990; 1992; Slep \& O’Leary, 1998). In daily caregiving experiences, children's characteristics and behavior influence parents' emotions, in turn, shaping parents' attributions. For example, when parents believe a child should be able to perform at a certain level due to either their age or a certain level of competence and children do not, parents become frustrated and respond in negative ways (Bugental, 1987; Dix, 1991; Dix, Ruble, Grusec, \& Nixon, 1986; Dix et al., 1989; Slep \& O'Leary, 1998). As noted above, this study examined parental attributions on a single 
continuum of internal (e.g., a child's behavior was dispositional, intentional) versus external attributions (e.g., a child's behavior was due to situational factors and not intentional; Coplan et al., 2002; Hastings \& Rubin, 1999; Mills \& Rubin, 1990; 1992). When parents experience frustration at children's misbehaviors when they believe the child knowingly misbehaved or it was the result of a dispositional trait in the child, parents report more internal attributions (Dix et al., 1986; Slep \& O’Leary, 1998). Thus, while distal influences on parental attributions are important, proximal influences on these cognitions can independently influence parents in meaningful ways.

\section{Tripartite Model of the Impact of the Family on Children's Emotion Regulation} and Adjustment

As previously noted, in addition to examining the antecedents of emotion socialization (i.e., children's temperament and maternal attributions) outcomes associated with emotion socialization will also be examined. Better put, the influence of parental emotion socialization responses on children's cognitive reappraisal, expressive suppression, and regulatory emotional self-efficacy will be investigated. The process by which parents affect their children's emotional development may best be understood via the tripartite model of the impact of the family on children's emotion regulation and adjustment (Sheffield Morris et al., 2007). Sheffield Morris and colleagues (2007) proposed three ways parental behaviors impact children's emotional outcomes. First, children's observation and modeling of parental emotion displays and interactions with others shape emotion outcomes. Parents' emotion displays implicitly teach children about what emotions are accepted and expected in the family, as well as how emotions should be regulated (Sheffield Morris et al., 2007). Children observe parents' patterns of emotion expression and regulation to learn how to respond in emotion-related situations and then repeat 
parental behaviors modeled to them by parents (Sheffield Morris et al., 2007). The effectiveness of parental modeling and children's observation is affected by the types of emotions directed at them by parents. Frequent displays of intense anger toward children inhibits learning about emotions and leads to emotional overarousal (Sheffield Morris et al., 2007). In addition, when the range of emotions and responses to emotions parents express is wider, children benefit (Sheffield Morris et al., 2007).

Second, emotion socialization is influenced by the emotional context of the family (Sheffield Morris et al., 2007). Emotion socialization processes do not take place in isolation, but are influenced by the larger emotional context of the family. Sheffield Morris and colleagues (2007) posit the emotional climate of the family is composed of four components (1) overall predictability and emotional stability of the environment, (2) parents' expectations and demands for maturity, (3) the amount of positive emotions expressed in the family, and (4) the amount of negative emotions expressed in the family. The emotional climate of the family determines the level of security a child feels to express their emotions and a child's understanding (or lack of understanding) regarding what is accepted and expected emotionally (Sheffield Morris et al., 2007).

Third, parental emotion-related parenting practices shape children's emotion outcomes. Parenting practices refer to specific parental behaviors defined by content and the parent's socialization goals (Sheffield Morris et al., 2007). Certain emotion-related parenting practices have particular relevance for children's emotion outcomes, including (1) emotion-coaching behaviors and parents' philosophy about emotions and children's emotional expressions (Gottman et al., 1996), (2) parental reactions to children's emotions, (3) parental encouragement of emotions and parent's perceived control over their child's emotions, (4) parents' teaching 
about strategies for regulating emotion, and (5) parents' selection or avoidance of opportunities for children to experience emotions (referred to as niche-picking).

While emotion socialization is clearly a multi-faceted process composed of multiple types of parental behaviors (Eisenberg et al., 1998; Sheffield Morris et al., 2007), the proposed investigation will be informed by and focus on examination of parents' reactions to children's negative emotions. During children's everyday experiences, there are multiple opportunities to experience both positive and negative emotions, and these experiences provide rich socialization opportunities for parents to directly respond to these displays (Eisenberg et al., 1998). Thus, the proposed investigation will focus on parental reactions to children's negative emotions.

\section{Summary of Theoretical Frameworks}

In sum, theories regarding the parent-child relationship and parental attributions frame this study, including transactional theory, parental attribution theory, and the tripartite model of the impact of the family on children's emotion regulation and adjustment. Although this study will be correlational and not longitudinal in nature, these theoretical perspectives place the parent-child relationship in transactional context and effectively describes the dynamic nature of the parent-child relationship, and the importance of attributions within the relationship. Using these theoretical frameworks as a guide, this study examined how children's temperamental traits are related to maternal emotion socialization responses, and the potential indirect role child temperament via parental attributions may play in this relationship. In addition, based on Sheffield Morris and colleagues' (2007) tripartite model, the proposed investigation examined how maternal emotion socialization responses may moderate the influence of child temperament on children's cognitive reappraisal, expressive suppression, and regulatory emotional selfefficacy. 


\section{Overview of Emotion Socialization}

Emotion socialization processes encompass parental responses that teach children about emotions and emotion-related behaviors within the context of the family (Eisenberg et al., 1998; Sheffield Morris et al., 2007). Emotion socialization processes begin at birth and continue throughout childhood and adolescence (see Denham, Bassett, \& Wyatt, 2015 for a review; Sheffield Morris et al., 2007). In addition, emotion socialization processes can be either direct or indirect (Eisenberg et al., 1998; Saarni, 1985; Saarni \& Crowley, 1990). Direct emotion socialization processes directly reinforce a child's emotion expression (Saarni \& Crowley, 1990). These responses include mothers' contingent responses to children's emotions and direct instruction given to the child related to emotions and how they are expressed (Saarni \& Crowley, 1990). On the other hand, indirect emotion socialization processes involve factors that intervene between the initial emotion stimulus and the child's emotional experience that communicate parental beliefs, rules, and expectancies about emotions (Saarni, 1985; 1993). Indirect emotion socialization can be both verbal and nonverbal, and is constrained by the developmental characteristics of relationship partners (Saarni, 1985).

Emotion socialization processes are heavily involved in the development of children's emotional outcomes. As described above, emotion regulation strategy use is one of the primary outcomes resulting from emotion socialization (Sheffield Morris et al., 2007), and emotion regulation influences multiple domains of children's psychosocial adjustment (e.g., academically, Graziano, Reavis, Keane, \& Calkins, 2007; socially, Monopoli \& Kingston, 2012). In addition, these processes play critical roles in teaching children about the meanings of emotions and when and how they are expressed (Denham et al., 2015; Eisenberg et al., 1998), promoting skills that help children express empathy, perspective-taking, and socially competent 
behavior (Izard, Fine, Schultz, Mostow, Ackerman, \& Youngstrom, 2001; Jones, Abbey, \& Cumberland, 1998).

Parent emotion socialization responses are typically examined as either an aggregate of responses to positive or negative emotions (O’Neal \& Magai, 2005). Parental emotion socialization behaviors are associated with children's outcomes. Generally, supportive emotion socialization responses (e.g., responses that validate a child's emotions and model healthy strategies for coping with and expressing emotion) are related to positive outcomes that reflect healthy emotional development, such as healthy emotion regulation and empathy (Blair et al., 2014; Taylor et al., 2013). On the other hand, nonsupportive responses (e.g., responses that minimize a child's emotional experience or punish the child for expressing negative emotions) are related to poorer outcomes for children, such as emotion dysregulation, psychological distress and internalizing difficulties (Buckholdt et al., 2014; Garside \& Klimes-Dougan, 2002; Silk et al., 2011).

\section{Operationalization of Emotion Socialization}

For the purposes of this study, direct parental emotion socialization responses will be examined using the O'Neal and Magai (2005) conceptualization of emotion socialization. This conceptualization identifies five parental responses to children's specific emotions. First, reward refers to supportive, comforting behaviors that help a child solve the problem or source of distress (e.g., showing physical affection to comfort the child). Punishment of a specific emotion refers to nonsupportive behaviors that discourage the expression of a specific emotion (e.g., telling the child they are being a baby for expressing the emotion). Neglect refers to a parent ignoring the child's display of emotion or not being available to help the child (e.g., being unavailable when the child is expressing a negative emotion). Parental override entails the parent 
squashing the child's emotion expression by either dismissing it or distracting the child from their expressed emotion (e.g., telling the child the source of distress is not a big deal). Magnify behaviors refer to parental responses that match the child's expressed emotion with the same amount or greater intensity. For this study, maternal emotion socialization responses will be aggregated into supportive and nonsupportive responses for each negative emotion individually. Studies with other emotion socialization instruments have collapsed individual emotion socialization responses into supportive and nonsupportive broadband factors as well (e.g., Fabes et al., 2002).

The nature of O’Neal and Magai’s (2005) approach to emotion socialization provides a more nuanced perspective regarding how parents respond to children's specific emotions, rather than positive or negative emotions only. While the aggregated positive-negative emotions approach has clear utility in preserving statistical power and promoting construct parsimony, it may not provide the most precise perspective on how parents are impacted by children's specific emotions. From the functional and psychoevolutionary perspectives of emotion, children's experiences of discrete emotions have distinct adaptive functions and produce distinct behavioral responses (Garside \& Klimes-Dougan, 2002; Izard \& Ackerman, 2000). In addition, these differing adaptive functions and behavioral responses encompass either approach or avoidance toward emotional stimuli and a goal that is being either facilitated or blocked (Smiley, Buttitta, Chung, Coffey, Wang, \& Borelli, 2016). For example, the experience of sadness or shame is related to avoidance or goal blocking behaviors (Izard \& Ackerman, 2000; Kelley, Brownell, \& Campbell, 2000). On the other hand, anger can lead the child to approach or avoid emotional stimuli, depending on contextual factors such as the child's sensitivity to threat and rewards (Carver \& Harmon-Jones, 2009; Cooper, Gomez, \& Buck, 2008). Taken together, these 
differences in behavioral activation patterns suggest that the experiences of different emotions precede differing behavioral and physiological patterns of responding (Huebner \& Izard, 1988; Izard \& Ackerman, 2000).

From the perspective of parenting, children's displays of specific negative emotions also likely activate and elicit varying behavioral responses from parents. Important theoretical work in this area posits that children's emotion expressions serve as catalysts for parents' emotional experiences and responses during parent-child interactions (Belsky, 1984; Dix, 1991). In addition, research also demonstrates parents clearly differentiate between and respond differently to children's displays of sadness, anger, and physical distress as early as infancy (Heubner \& Izard, 1988). In these parent-child interactions, anger, for example, has the potential for being the most behaviorally activating (i.e., it mobilizes the mother to exercise resources on the child's behalf; Izard, 1978), whereas sadness is related to more passive maternal responses because mothers believe a child can cope with it independently (Huebner \& Izard, 1988). This perspective is congruent with the approach-avoid framework discussed above. For example, sadness, shame, and fear lead a child to withdraw from a particular emotional stimuli, and as a result, parents would be in less direct need to respond to the child's emotion display. On the other hand, if a child was feeling angry and potentially acting in an aggressive and defiant manner, that would require a more pressing parental response.

While most emotion socialization research examines children's emotions from the perspective of positive and negative emotions, there are some studies that have examined discrete emotions in parenting processes generally and emotion socialization processes specifically (parenting, Heubner \& Izard, 1988; emotion socialization, Garside \& KlimesDougan, 2002; Hastings, Klimes-Dougan, Kendziora, Brand, \& Zahn-Waxler, 2014; Klimes- 
Dougan et al., 2007; Root \& Rasmussen, 2015). Taken together, this approach will provide important nuanced evidence about potential differences in maternal emotion socialization responses to children's specific emotions.

\section{Child Effects on Socialization, Parenting, and Emotion Socialization}

While the emotional and social outcomes associated with specific emotion socialization responses have been well-studied, the antecedents of emotion socialization have are less studied. Children play an active role in constructing their socialization experiences (Bell \& Chapman, 1986; Belsky, 1984; Bugental \& Grusec, 2006; Maccoby, 2015). Child characteristics are particularly influential in shaping parental socialization, particularly children's negative emotionality (Sheffield Morris et al., 2007). Children's temperamental emotionality and other temperamentally-based regulatory differences can make them "more or less difficult to care for" (Belsky, 1984, p. 86) and influence the emotions children experience and the intensity at which they experience them (Brumariu \& Kerns, 2013). Although the evidence is mixed, the literature demonstrates some children are more susceptible to experience negative emotions than others based on temperamental differences (Bates, Schermerhorn, \& Petersen, 2012; Laukkanen et al., 2014; Thomas \& Chess, 1977). Children's dispositional emotionality and emotional patterns impact how parents think and feel and are related to negative parenting outcomes in early and middle childhood (Dix, 1991; Lipscomb, et al., 2011; Paulussen-Hoogeboom et al., 2008; van der Bruggen et al., 2010). For example, mothers of preschool-aged children high in negative emotionality are more intrusive during parenting interactions and engage in more authoritarian behaviors (Paulussen-Hoogeboom et al., 2008; Porter et al., 2005). In middle childhood, high levels of child negative emotionality positively predict parental psychological and behavioral control (Barber, Stolz, \& Olsen, 2005; Laukkanen et al., 2014). Thus, negative emotionality and 
other regulatory difficulties influence the effectiveness of parenting behaviors and shape parents' responses to children (Scaramella \& Leve, 2004). In particular, temperamental traits such as negative emotionality frustrate parents and evoke more negative, controlling, and angry parenting behaviors (Porter et al., 2005; Scaramella \& Leve, 2004).

Children's traits not only shape general parenting experiences, but also specific domains of parenting, including emotion socialization processes. As described above, children's emotional display patterns and emotion regulation efforts shape parental emotion socialization behaviors (Dix, 1991; Sheffield Morris et al., 2007). For instance, if a child experiences a negative emotion and is unable to regulate effectively, this is likely to impact the ways their parents respond to these displays (Sheffield Morris et al., 2007). Existing evidence demonstrates these relations between children's emotion regulation and parental emotion socialization. For example, poor emotion regulation in middle childhood is linked to use of parental nonsupportive emotion socialization practices (Eisenberg \& Fabes, 1994) and more effective child coping (a component of emotion regulation) is related to the use of supportive emotion socialization practices (Eisenberg, Fabes, \& Murphy, 1996). In addition, mothers who perceive their adolescent as being more anxious (i.e., having difficulty regulating emotions) are more likely to use nonsupportive emotion socialization practices in response to their child's negative emotions (e.g., shutting down the child's emotional display, ignoring the child's distress; Suveg, Zeman, Flannery-Schroeder, \& Cassano, 2005). Taken together, child temperament influences emotions socialization in similar ways that it influences general parenting practices.

Similarly, children's dispositional traits affect parental attributions. For instance, child difficultness (e.g., being difficult to manage or being frustrating to the parent) influences parental 
attributions. Mothers of children perceived as difficult (Gretarsson \& Gelfand, 1988) or aggressive (Dix \& Lochman, 1990) make more internal attributions of negative social behaviors. Parents are also affected by children's age (Dix et al., 1989; Dix et al., 1986). As children get older, parents attribute normal violations of social norms significantly more as being dispositionally-based and the child engaged in the behavior intentionally (i.e., indicative of internal attributions; Dix et al., 1986). As children's age increases, mothers believe children have a greater capacity to understand their misbehaviors, have greater knowledge about what they should have done, and deserve more responsibility for the misbehavior. This suggests that mothers' attributions shift from being externally-oriented when children are young, but as children age, mothers' expectations change and they attribute misbehaviors as being more under the child's control (Dix et al., 1989).

Thus, it is likely that negative emotionality and related regulatory traits (i.e., soothability, attention focusing, and inhibitory control) are perceived differently as children age. Mills and Rubin (1992; 1990; Mills, 1998) and their colleagues (Hastings \& Rubin, 1999) have examined the influence of children's temperamentally-based aggression, social withdrawal, and fearfulness on mothers' attributions. In early childhood, mothers report more external attributions (e.g., the behavior resulted from the child being tired) for children's aggressive and socially withdrawn behaviors (Hastings \& Rubin, 1999). As children age, mothers report fewer external attributions for both aggressive and withdrawn behaviors and more internal ones for social withdrawal (Mills \& Rubin, 1990; 1992).

Examination of temperament specifically during late childhood is also important. As children age, parental expectations for behavior increase, and violations of these expectations are increasingly related to more power assertive and punitive parental responses (Dix et al., 1986). 
Because parents respond so negatively to children who violate expectations for behavior, examining children's temperamental differences in emotionality and regulatory patterns can help identify children who are the most at-risk to receive maladaptive parenting or develop psychopathologies related to emotion regulation (Campbell-Sills, Ellard, \& Barlow, 2014; Paulussen-Hoogeboom et al., 2008; Porter et al., 2005). Taken together, child effects are an important influence on parental attributions during late childhood.

\section{Parenting Effects on Socialization, Parenting, and Emotion Socialization: Attributions}

While children clearly contribute to socialization processes, parental socialization responses are also constantly influenced by a range of both distal and proximal parent-level factors as well. Distally, for example, parents' socialization decisions are clearly impacted by their own personality (Belsky, Crnic, \& Woodworth, 1995; Puff \& Renk, 2016) and emotional difficulties they experience (Breaux, Harvey, \& Lugo-Candelas, 2016). In addition, parental and family stress and daily hassles also clearly shape parents' socialization decisions (Belsky et al., 1995; Conger \& Conger, 2002; Conger, Ge, Elder, Lorenz, \& Simons, 1994; Neppl, Senia, \& Donnellan, 2016).

From a more proximal perspective, parental socialization efforts are also strongly influenced by what parents are thinking and feeling in the moment (Dix, 1991; Bugental \& Grusec, 2006). Parental cognition is a multi-faceted process and takes place at multiple levels of cognitive awareness (Bugental, 1992; Bugental \& Johnston, 2000; Grusec et al., 1994). The sequence of parental cognitions begins with automatic cognition that unconsciously accesses existing caregiving schemas without explicit effort (Bugental, 1992). Following this automatic appraisal, cognitions become controlled, and are conscious and deliberate, allowing parents to reflect upon caregiving situations and then decide how they will react (Bugental, 1992). 
Parental cognitions are multidimensional; Bugental and Johnston (2000) identified four specific types of parenting cognitions, including (a) descriptive cognitions (i.e., thoughts about the way things are perceived in a family), (b) evaluative-prescriptive cognitions (i.e., thoughts about the way things should be in a family), (c) efficacy cognitions (i.e., thoughts about the way things are and should be in a family), and (d) analytical cognitions (i.e., thoughts about reasons for family events). These cognitions are dependent on parents' larger belief systems about parenting and childrearing. These belief systems include factors such as the parent's sense of self-efficacy as a parent, the level of control they attribute to themselves and their child, their perceived power in influencing their child, and their cultural context (Bugental \& Johnston, 2000; Grusec, Hastings, \& Mammone, 1994; Mills, 1998).

Parental attributions are an indirect form of emotion socialization and a component of parental cognitions that guide parental behaviors. (Dix et al., 1989; Mills \& Rubin, 1990; 1992; Slep \& O’Leary, 1998). Parental attributions are a form of analytical cognitions and are a cognitive schematic structure composed of parents' memories and experiences with their child, their parents, and in other important relationships that provides information about how to respond in caregiving situations (Bugental, 1987; Bugental \& Happaney, 2002; Dix, 1993; Grusec, Adam, \& Mammone, 1993; Grusec et al., 1994). Specifically, attributions attune parents to specific aspects of caregiving situations, such as the intentionality of a child's behavior, the valence of the child's intent (e.g., positive, negative, or neutral), balance of control between parent and child, and responsibility for a particular behavior (Grusec et al., 1994; Slep \& O’Leary, 1998). The foundation for parental attributions is rooted in parents' own experiences with their parents as children, and their attributions reflect the sense of control (or lack of it) they feel in interactions with others as a result of these early experiences (Grusec et al., 1994). These 
attributions shape parents' behavioral, cognitive, and emotional responses to caregiving situations (Grusec et al., 1994; Mills, 1998). When parents are in a caregiving situation where a response is needed, they draw on this knowledge base to guide their behavior ("this behavior happens because of X, so I will do Y to respond to it”; Bugental \& Happaney, 2002).

Relations between aspects of parental attributions noted above (i.e., intentionality, balance of control, and responsibility) and parenting behaviors are established. In regard to parents' attributions of children's intentionality in behaviors, when parents believe their child is intentionally engaging in a misbehavior and has negative intent, parents respond with more anger and overreact in their discipline response to the misbehavior (Slep \& O'Leary, 1998). In terms of balance of control, parents who attribute higher levels of control to children rather themselves are more emotionally reactive and respond more negatively to hypothetical and actual caregiving situations, particularly in reference to a difficult child (Bugental, 1992; Bugental et al., 1990). In addition, parents who attribute low levels of control to themselves engage in more authoritarian parenting, overprotective behaviors, and inconsistent parenting when children are more fearful (Mills, 1998). Finally, child-centered responsibility attributions (i.e., the child was responsible for the particular behavior) are related to higher levels of observed and self-reported overreactive parenting behaviors (Slep \& O’Leary, 1998).

Children's behaviors elicit emotional responses from parents (Dix, 1991). Given that parental emotion socialization responses take place in response to children's emotions, it is very likely that these displays in particular will activate parents' own emotions (e.g., anger at a child's outburst of negative emotion) and demand a response from the parent. These displays create the likelihood that parents will need to draw upon their attributions and understanding of the behavior to respond to their child. Thus, parents' attributions for children's behaviors may be an 
important predictor of specific types of parenting, such as emotion socialization, though this has not yet been tested empirically.

Parental attributions during late childhood. By late childhood, parents have had extensive experiences interacting with their children and, as a result, created an extensive knowledge structure of attributions to draw from in making socialization decisions (Bugental \& Happaney, 2002; Dix et al., 1989; Slep \& O’Leary, 1998). Whereas with younger children, parents are more flexible about children's misbehaviors because they understand children do not have full control over their behavior with older children, parents are much more reliant on attributional information to help them understand the source of a particular behavior so they can make effective socialization decisions (Dix et al., 1989). Compared to other types of parental cognitions (e.g., efficacy cognitions about the way things are or should be in a family) parental attributions of children's behaviors tap into a foundational, basic question parents ask in socialization interactions, namely, where does a particular behavior come from? In this way, parental attributions represent parents first line of defense in understanding the source of a behavior, and subsequently, how best to address it in-the-moment and in the future (Bugental \& Happaney, 2002).

\section{Emotion Socialization and Children's Emotion Outcomes}

Parental emotion socialization shapes children's emotion outcomes. As noted above, children's emotion regulation is influenced by parents' emotion socialization practices (Blair et al., 2014; Sheffield Morris et al., 2007). Indeed, it is well-documented that parental emotion socialization responses shape children's emotion regulation. Emotion socialization responses that minimize or punish a child for expressing emotions lead to poorer regulation and emotion dysregulation (Buckholdt et al., 2014). In contrast, supportive responses that encourage children 
to express their emotions and model effective emotion regulation are associated with effective emotion regulation (Blair et al., 2014). While much is known about emotion regulation, it is important to understand how emotion socialization may also influence other aspects of children's emotional functioning. One aspect of children's emotional functioning may be children's selfefficacy to regulate their own emotions.

Stemming from Bandura's social cognitive theory (2001) self-efficacy is a critical cognitive component of human agency and has a powerful influence on individual behavior and functioning, underlying motivation of responses to environmental stimuli (Caprara, 2002). Without self-efficacy beliefs, individuals have little motivation to act, and self-efficacy beliefs have a powerful influence on the types of situations individuals enter (e.g., "if I think I can do this, I'll try X") and the way they view these situations (e.g., "I think X is something possible I can achieve"; Bandura, 2001). For example, an individual with more positive, affirming selfefficacy beliefs may view a challenging situation more positively (e.g., "this is hard, but I think I can do this") than someone with less positive or even negative self-efficacy beliefs, who will likely view the same situation in a negative, self-hindering way (e.g., "I know I can't do it, so I'm not even going to try"; Bandura, 2001).

Self-efficacy beliefs are a proxy for the self-reflective component of an individual's personality (Caprara, 2002). Personality is both a construction of external influences and an agentic system controlled by the individual (Caprara, 2002). Self-reflective processes allow an individual to consider their competencies and characteristics, forming organized systems of selfefficacy beliefs (Caprara, 2002). Self-efficacy can be measured as a global construct, but nuances exist between individuals' self-efficacy beliefs in different domains. Regulatory emotional selfefficacy is a domain-specific form of self-efficacy that refers to an individual's beliefs regarding 
their ability to manage basic positive and negative states (Caprara et al., 2003). Given the critical importance of emotion regulation and its relation to adjustment, individuals' own beliefs regarding their ability to manage these emotions are crucial because they shape children's regulatory efforts in response to negative emotions (Bandura et al., 2003; Hu, Zhang, Wang, Mistry, Ran, \& Wang, 2014; Sundermann \& DePrince, 2015).

In addition, self-efficacy beliefs undergird children's regulatory responses. A sense of high regulatory self-efficacy shapes a child's regulatory effort in the face of negative emotions and provides varying degrees of motivation preceding effort (or lack of it). For example, if a child possesses high regulatory self-efficacy, they are much more likely to initiate and use regulatory strategies. Conversely, if a child has low regulatory self-efficacy and does not believe they can effectively regulate, they are much less likely to exert regulatory effort and more likely to use ineffective regulatory strategies they know will be ineffective (Bandura et al., 2003).

As noted above, self-efficacy to regulate emotion is a critical antecedent of children's emotion regulation efforts and is a particularly important cornerstone of behaviors and responses that require effective emotion regulation (Bandura et al., 2003; Caprara, 2002). For example, if a child is well regulated or feels efficacious to regulate negative emotions, this reduces the likelihood the child will display dysregulated expressions of emotion or exhibit behaviors associated with a lack of regulation of negative emotions (e.g., internalizing or externalizing difficulties; Bandura et al., 2003; Buckholdt et al., 2014; Joormann \& Vanderlind, 2014; Sundermann \& DePrince, 2015). In addition, emotion regulation efforts also help children to act more effectively in social situations (Calkins \& Dollar, 2014), which is likely to increase the child's sense of efficacy in future social situations (Bandura et al., 2003; Caprara, 2002). 
Parental emotion socialization responses that punish, dismiss, or ignore children's emotional displays may communicate to a child either verbally (e.g., "stop crying, it's not a big deal") or implicitly (e.g., responding with negativity or hostility) that they are overly emotional or unable to regulate their emotions effectively, putting a child's sense of regulatory emotional self-efficacy in the future at-risk. On the other hand, when parents engage in supportive emotion socialization behaviors that validate children's emotions and teach children strategies to effectively regulate their emotions, these behaviors will likely increase a child's sense of competence, increasing their regulatory emotional self-efficacy.

Given the construct of regulatory emotional self-efficacy is fairly new, the evidence documenting links between children's regulatory emotional self-efficacy and adjustment outcomes is limited. However, the existing evidence with adolescents indicates that higher levels of self-efficacy to regulate negative emotions is related to lower incidences of poor outcomes, such as current and future depression and current delinquency (Bandura et al., 2003). In addition, higher levels of self-efficacy to regulate both positive and negative emotions are related to social forms of self-efficacy (e.g., feeling confident being a relationship partner) and higher levels of both positive thinking and happiness (Caprara et al., 2006). Indeed, these findings suggest that feelings of self-efficacy to regulate various types of emotions are important in buffering against the incidence of developmental risk factors and in promoting positive outcomes. These benefits suggest the developmental importance of examining regulatory self-efficacy and exploring how parental emotion socialization responses may impact its development.

\section{Significance of the Proposed Study}

As aforementioned, the aims of this study examined the relations between children's temperament, maternal attributions of specific negative emotion displays, maternal emotion 
socialization responses, and children's emotion-related outcomes, including children's cognitive reappraisal, expressive suppression, and regulatory emotional self-efficacy. Inherent in the examination of these relations is the notion that better understanding of the antecedents of emotion socialization will provide understanding about aspects of children and parents that promote the well-being of children, or conversely, factors that can put a parent-child dyad atrisk. Furthermore, given parental emotion socialization responses are associated with children's psychosocial adjustment, it is particularly prudent to better understand antecedents of maternal emotion socialization that shape these processes (Buckholdt et al., 2014; Hurrell et al., 2015).

Better understanding of the ways that parents contribute to children's emotion regulatory ability is particularly important because emotion regulation allows children to attend to and manage their emotional experiences (Eisenberg et al., 2007). Parental emotion socialization shapes the choices children make when they need to regulate emotions during the later childhood and preadolescent years (Buckholdt et al., 2014). In turn, these emerging emotion regulation processes and skills undergird healthy child outcomes in domains of mental health (Ford, Mauss, Smolen, Troy, Smole, \& Hankin, 2014; Kim \& Cicchetti, 2010; Silk, Shaw, Forbes, Lane, \& Kovacs, 2006), social adjustment (Monopoli \& Kingston, 2012; Rubin, Bukowski, \& Parker, 1998), and academic adjustment (Graziano et al., 2007; Gumora \& Arsenio, 2002).

Adaptive emotion regulation also protects against both internalizing and externalizing difficulties (reported by self, teacher, and mother; Ford et al., 2014; Kim \& Cicchetti, 2010; Silk et al., 2006). For instance, the habitual use of certain cognitively-based emotion regulation strategies (i.e., strategies that take place internally, such as reappraising a distressing situation) protects against the negative effects of life stress on emotional and behavioral problems (Flouri \& Mavroveli, 2012). The use of maladaptive emotion regulatory strategies and the inability to 
regulate emotions are related to internalizing and externalizing problems, including anxiety disorders (e.g., ruminating on the source of distress; see Jacob, Thomassin, Morelen, \& Suveg, 2011; Mennin, Fresco, Ritter, \& Heimberg, 2015), depression (Joormann \& Vanderlind, 2014; Sundermann \& DePrince, 2015) and antisocial behaviors (Buckholdt et al., 2014).

Although the relation between emotion regulation and psychosocial adjustment is complex, ineffective emotion regulatory ability often precedes poor adjustment. For example, rumination, a maladaptive emotion regulatory strategy, cognitively focuses the individual's mind on the negative emotion they are experiencing and prolongs the experience of negative emotions (Joormann \& Vanderlind, 2014; Yan, Han, \& Li, 2016). This is associated with the likelihood or maintenance of a depressive episode (Joormann \& Vanderlind, 2014). In addition, children who experience anxiety are more likely to use maladaptive emotion regulation strategies such as overly focusing on a negative emotion (i.e., rumination) or attempting to shut down the experience of a negative emotion (i.e., expressive suppression) in an effort to avoid feelings of worry or distress (Jacob et al., 2011; Mennin et al., 2015). For example, efforts to suppress the expression of negative emotions may increase the internal affective experience of negative emotions because of the ongoing cognitive effort required to suppress such expressions (Gross, 1998; Gross, 2002). If effective regulatory skills are not used, appropriate cognitive and emotional resources cannot be directed toward managing distress, which maintains or exacerbates anxious feelings (Jacob et al., 2011).

Emotion regulation skills are also an important element of children's social relationships (Bell \& Calkins, 2000; Parke, 1994). Emotional experiences simultaneously initiate and utilize multiple psychological processes (e.g., appraisal, attention; Calkins \& Dollar, 2014). If a child chooses poor emotion regulations strategies that do not effectively regulate the emotion they are 
feeling, important neurological resources are constantly directed toward that emotion. As a result, the amount of neurological and psychological resources to address social demands, act flexibly, and inhibit behaviors that might impact how peers view them are decreased (Kim \& Cicchetti, 2010). Thus, when children are poorly regulated, they have fewer resources to initiate, motivate, and organize socially adaptive behaviors with peers (Berkovits \& Baker, 2014; Cicchetti, Ackerman, \& Izard, 1995; Rydell, Thorell, \& Bohlin, 2007). However, when children are well regulated, cognitive processing can be directed toward more immediate social and academic tasks (Calkins \& Dollar, 2014). In these situations, children are better able to attend to what happens in their environment and act in emotionally and socially competent ways (Calkins \& Dollar, 2014). As a result, well-regulated children engage in behaviors that do not aggravate peers but rather make children more desirable and socially competent (e.g., helping behaviors, understanding others' emotions, perspective-taking; Lopes, Mestre, Guil, Kremenitzer, \& Salovey, 2012; Monopoli \& Kingston, 2012; Rubin et al., 1998).

Furthermore, it was particularly important to examine these relations in a sample of older (ages 10-12) children. Parents continue to play an important role in emotion socialization processes during the middle childhood years (Zeman, Perry-Parrish, \& Cassano, 2010). Furthermore, parents' expectations of children's behavior increase as children age, and parents make more internal attributions in older children compared to younger ages (Dix et al., 1986; Dix et al., 1989; Mills \& Rubin, 1992). Internal attributions are related to more negative parenting behaviors such as responding with more anger and over reactive discipline (Slep \& O'Leary, 1998). In addition, parents' attributions are subject to multiple biases (e.g., negative mood; Nelson, O’Brien, Calkins, \& Keane, 2013). As a result, a parent could potentially wrongly attribute a child's negative behavior to internal (e.g., dispositional or trait-like causes). If they do 
so, they are more likely to respond to their child in negative ways (e.g., punishing the child; Dix et al., 1989). As a result, a child does not receive supportive socialization opportunities that might benefit them in the future (Dix et al., 1989). Furthermore, children act in ways that are consistent with their parents' behaviors toward them, for better for worse (Bugental \& Shennum, 1984; Dix, 1993; Dweck, 1975). If a parent's behavior is reflective of highly internal attributions, they may respond to their child with more anger and frustrating, eliciting dysregulated or frustrating behaviors from the child (Bugental \& Shennum, 1984; Slep \& O’Leary, 1998).

In terms of children's emotion regulation and self-efficacy outcomes during later childhood, this period is particularly formative. Children experience profound physical, social, educational, and familial changes during the transition to early adolescence, including the transition from elementary school to middle school or junior high (Eccles et al., 1993; Granic, Hollenstein, Dishion, \& Patterson, 2003; Hollenstein \& Lougheed, 2013; Levitt et al., 2005; Lippold, Powers, Syvertsen, Feinberg, \& Greenberg, 2013). In addition, the mismatch between early adolescent children's developmental needs and the transition to middle school or junior high makes matters significantly more difficult for children (Eccles et al., 1993). For example, during the transition middle school or junior high, academic standards increase for children, and there is a significantly higher emphasis on maintaining control and discipline in the classroom rather than facilitating learning (Midgley, Feldlaufer, \& Eccles, 1988). Furthermore, during this transition, levels of academic and social support children receive from teachers drastically decreases, further perpetuating the lack of motivation and social isolation many children feel (Duchesne, Ratelle, Poitras, \& Drouin, 2009; Eccles, 1994; Roeser \& Eccles, 1998; Seidman, Allen, Aber, Mitchell, \& Feinman, 1994). This is particularly important to consider given the age group examined in this study is on the cusp of making this transition. As a result, children in this 
age group are vulnerable to academic, social, and emotional difficulties, and emotional problems during this period can last through adulthood (Kansky, Allen, \& Diener, 2016; Roeser \& Eccles, 1998).

From an emotion regulation perspective, this time period is also particularly formative. While children do experience gains in emotion regulatory ability during later childhood (Rawana et al., 2014), they are still in need of support as they form and practice cognitive reappraisal skills that will be used during the transition to adolescence and throughout adulthood (KlimesDougan et al., 2007). In addition, older children possess more cognitive skills that may increase the likelihood they would attend to, remember, and integrate parental messages about their traits, including temperamental ones that promote or undermine their emotion regulation (i.e., negative emotionality, soothability, attention focusing, and inhibitory control). For example, during the older childhood years, children experience gains in the amount of working memory available to them (Kail, 1993), their ability to selectively attend to specific stimuli (Tabibi \& Pfeffer, 2007), and children's long-term memory becomes more established (Schneider, 2002). In addition, older children begin to engage in metacognition, reflecting upon their own thinking and being able to engage in mental inferences (Miller et al., 2003). These skills help children attend to influential parental messages more effectively, but they may lack the skills to reframe hostile or negative messages about emotionality. As a result, a child's psychosocial adjustment is particularly at-risk if they are given inaccurate or negative messages about themselves that are remembered, stored, and internalized. This may also be particularly problematic for this age group as they are approaching the transition to adolescence, where effective emotion regulation and coping strategies are needed to navigate the multitude of changes associated with it (Forrest, Bevans, Riley, Crespo, \& Louis, 2013; Graber \& Brooks-Gunn, 1996). Taken together, later 
childhood is a particularly formative period for children's emotional trajectories, including their emotion regulation and regulatory emotional self-efficacy.

\section{The Proposed Study}

To date, the majority of the research in this area has focused on outcomes of parental emotion socialization and to a more limited extent, child-level antecedents of these behaviors. Given the parent-child socialization relationship is affected by both children and parents (Kuczynski \& Parkin, 2007), it is particularly important to examine both child- and parent-level factors that may precede emotion socialization processes. Examining the ways different dimensions of children's temperament affect parental attributions during this period is important. In addition, it is particularly crucial to acknowledge the contribution of parental cognitions to these processes. While children themselves do much to contribute to the types of responses parents give to children's negative emotions, not including parents' cognitions as a factor in these responses oversimplifies parents' contribution and does not fully describe the complexity of parents' potential to make meaning of their experiences as children themselves (Grusec et al., 1994) or as parents, in long-term interaction with their own child (Kuczynski \& Parkin, 2007; 2009). Indeed, parents' cognitions, particularly their attributions, shape the ways parents respond to their children (Dix et al., 1989; Mills, 1998; Slep \& O’Leary, 1998), suggesting that understanding the ways these cognitions lead parents to act more or less supportively is particularly important.

In addition, the research in this area demonstrates that emotion socialization behaviors can either promote or inhibit the development of effective emotion regulation in children (Buckholdt et al., 2014). However, there is a relative paucity examining the contributions of temperament and emotion socialization to the development of emotion regulatory strategies 
during late childhood. Given the importance of emotion regulation during the late childhood years and the emotional resources and abilities needed for the transition to adolescence (Eccles et al., 1993; Granic et al., 2003; Hollenstein \& Lougheed, 2013; Levitt et al., 2005; Lippold et al., 2012) it is important to better understand how to foster the development of children's adaptive emotion regulatory strategies. In addition, because so many resources are needed to make and navigate the transition to adolescence, it is important to understand factors that precede children's regulatory efforts, particularly children's regulatory emotional self-efficacy. As aforementioned, children's sense of self-efficacy supports or impedes actual regulatory efforts (Caprara, 2002; Caprara et al., 2008). Understanding the specific ways parents' emotion socialization behaviors promote or undermine children's self-efficacy can help increase the use of adaptive parental emotion socialization responses and identify parental behaviors that are particularly harmful to children's developing regulatory emotional self-efficacy.

In sum, the aims of this study were threefold. The first aim was to examine how children's negative emotionality, soothability, attention focusing, and inhibitory control predicted maternal attributions of children's expressions of dysregulated negative emotions. The second aim was to investigate whether children's temperament indirectly affected maternal supportive and nonsupportive responses to specific emotions via maternal attributions of each emotion. Finally, the third aim of this study was to examine whether maternal emotion socialization responses moderated the relation between children's negative emotionality, soothability, attention focusing, and inhibitory control and their cognitive reappraisal, expressive suppression, and regulatory emotional self-efficacy.

\section{Hypotheses}

Three main research questions and corresponding hypotheses are outlined below. 


\section{Research question one: Child temperament predicting maternal}

attributions of specific emotion displays. The first research question examined how the four dimensions of temperament (i.e., negative emotionality, soothability, attention focusing, and inhibitory control) were related to maternal attributions of sadness, anger, fear, and shame/embarrassment. The attributions scale used herein was a continuous measure, with high scores indicating external attributions and lower scores indicating internal attributions. Thus, based on evidence linking children's difficultness and internal attributions (Bugental \& Shennum, 1984; Bugental et al., 1990; Gretarsson \& Gelfand, 1988), it was hypothesized that children's negative emotionality would be negatively related to low levels of attributions (i.e., external) for each emotion, and soothability, attention focusing, and inhibitory control would positively related to each.

\section{Research question two: Indirect effects of child temperament on maternal emotion} socialization of specific emotions via maternal attributions of specific emotion displays. The second research question examined whether child temperament (i.e., negative emotionality, soothability, attention focusing, and inhibitory control) indirectly affected supportive and nonsupportive emotion socialization via maternal attributions of sadness, anger, fear, shame/embarrassment (see Figure 1). Previous work demonstrates other temperamental traits such as social withdrawal and aggression are increasingly related to internal attributions as children get older (Mills \& Rubin, 1992). It is not entirely clear how these components of children's temperament will affect parental attributions as opposed to other temperamental traits, but it is likely that parents might view more emotion dysregulation less positively, particularly in children of this age group (ages 10-12; Dix et al., 1986; Mills \& Rubin, 1992). In addition, it is likely that the association between these temperamental traits and maternal emotion socialization 
is not simply one-to-one. Based on work linking behaviors based in children's temperament to parents' attributions (Mills \& Rubin, 1992; Hastings \& Rubin, 1999) and parents' internal attributions to more negative parenting responses (Slep \& O'Leary, 1998), it was expected child temperament would be indirectly related to supportive and nonsupportive emotion socialization via maternal attributions of sadness, anger, fear, and shame/embarrassment. An indirect effect implies a significant relation between the temperament dimension $>$ maternal attributions relation $(\mathrm{x}>\mathrm{m}$ path) and the maternal attributions $>$ nonsupportive and supportive emotion socialization relation ( $\mathrm{m}>\mathrm{y}$ path; Mathieu \& Taylor, 2006).

\section{Research question three: Maternal emotion socialization as moderator}

between child temperament and emotion outcomes. The third research question examined the relation between the four components of emotionality and regulatory behaviors, parental emotion socialization of specific emotions, and the three emotion outcomes, cognitive reappraisal, expressive suppression, and self-efficacy to regulate negative emotions (see Figure 2). These three outcomes represent different components of children's emotion regulation repertoire, with cognitive reappraisal (i.e., cognitively reframing a distressing event to reduce distress or feel happier) and regulatory emotional self-efficacy being more positive outcomes, and expressive suppression being more negative (i.e., shutting down a distressing or positive emotional experience; Gullone \& Taffe, 2012). Specifically, the four dimensions of temperament (i.e., negative emotionality, soothability, attention focusing, and inhibitory control), maternal supportive and nonsupportive emotion socialization responses to sadness, anger, fear, and shame/embarrassment, and the interaction between them were examined as predictors of children's cognitive reappraisal, expressive suppression, and regulatory emotional self-efficacy. 
It is well established that aspects of children's temperament and emotion socialization predict children's emotion regulation (Eisenberg \& Fabes, 1994; Eisenberg, Fabes, Shepard, Guthrie, Murphy, \& Reiser, 1999). As a result, it was hypothesized that negative emotionality would be negatively related to cognitive reappraisal and self-efficacy to regulate negative emotions and positively predict expressive suppression. For soothability, attention focusing, and inhibitory control, it was expected these dimensions would be positively related to cognitive reappraisal and regulatory emotional self-efficacy and negatively related to expressive suppression.

In terms of emotion socialization responses, supportive responses promote emotion regulation while nonsupportive responses undermine it (Blair et al., 2014; Buckholdt et al., 2014). Therefore, across all specific emotions, it was hypothesized that supportive emotion socialization responses would be positively related to cognitive reappraisal regulatory emotional self-efficacy and negatively related to expressive suppression; moreover, it was predicted nonsupportive emotion socialization would be negatively related to cognitive reappraisal and regulatory emotional self-efficacy and positively related to expressive suppression.

While main effects for the temperamental traits and emotion socialization responses were predicted, it was also expected that these effects would be subsumed when the interaction terms were entered into the regression models. Indeed, it is likely that emotion socialization practices moderate the relation between dimensions of children's temperament and their emotion-related outcomes. The guiding principle behind these hypotheses is that supportive emotion socialization responses to sadness, anger, fear, and shame/embarrassment promote healthier emotion outcomes (i.e., cognitive reappraisal, regulatory emotional self-efficacy) for children and buffer 
against the use of expressive suppression. For example, in the case of negative emotionality, it was hypothesized that supportive emotion socialization to specific emotions would decrease the (negative) correlation between negative emotionality and cognitive reappraisal and regulatory emotional self-efficacy. For nonsupportive emotion socialization, these responses would hinder the use of these strategies, and increase this correlation. In the case of suppression, a similar pattern was hypothesized; supportive emotion socialization would buffer against the use of expressive suppression and decrease the (positive) correlation between negative emotionality and expressive suppression. Nonsupportive responses, in turn, were predicted to hinder children's emotional health, and increase this correlation.

The opposite pattern was hypothesized for the remaining three remaining temperamental variables, soothability, attention focusing, and inhibitory control. Because these three traits promote children's emotion regulation (e.g., Rothbart \& Bates, 2006) supportive emotion socialization responses promote the use of cognitive reappraisal and regulatory emotional selfefficacy, increasing the (positive) correlation between these variables. Nonsupportive responses, in turn, make it more difficult for children to utilize adaptive emotion regulation strategies, decreasing the (positive) correlation between these constructs and cognitive reappraisal and regulatory emotional self-efficacy. In terms of expressive suppression, the same pattern would apply; supportive responses to specific emotions would buffer against the use of expressive suppression, increasing the (negative) correlation between these dimensions of temperament and expressive suppression. Nonsupportive responses would hinder emotion regulation, decreasing the correlation. 


\section{Chapter 3}

\section{Method}

\section{Participants and Procedures}

One hundred and twenty-three mother-child dyads and 36 mothers were recruited locally from Monongalia County in the state of West Virginia and nationally through a study recruitment website. The final sample of 123 mother-child dyads and 36 mother participants resulted from the removal of mother-child dyads for one of the following reasons: (a) the child was adopted after 18 months (4 dyads), (b) the child was in a set of twins or the mother completed the survey for two different children and one was randomly chosen to be removed to prevent shared method variance (5 dyads), (c) one or both members of a pair completed the survey more than once (6 mothers, 1 child), (d) one or both members of a pair did not complete the surveys past clicking on the link or providing their name (14 mothers, 26 children), or (e) the child completed it alone and their mother did not complete it (4 children).

Mothers and children did not complete an identical battery of surveys. As will be discussed below, mothers reported on the four dimensions of child temperament and their attributions for children's dysregulated emotion displays. Children reported on their emotion regulation strategy use, regulatory emotional self-efficacy, and their mothers' emotion socialization responses. For research question one (i.e., child temperament as predictor of maternal attributions), mothers were the sole informants. Given that mothers were the sole informants for research question one, data for the 36 mothers who did not complete the surveys with their child (i.e., were not in a dyad) were added to the 123 mothers in dyads to maximize sample size. For research questions two and three (i.e., temperament indirectly affecting emotion socialization responses through maternal attributions and moderation of supportive and 
nonsupportive responses on relations between child temperament and emotion outcomes), both children and mothers were informants and, as a result, the data for the additional 36 non-dyad mothers could not be used, and the sample consisted of the 123 mother-child dyads.

Several methods were used to recruit mothers of children ages 10-12. For recruitment of mothers within Monongalia County, West Virginia, flyers advertising the study were placed in the community at local businesses, doctor's offices, and community centers. Social media was also used to advertise the study. An informational post about the study was posted and shared through the social media website, Facebook. Advertisements were also shared through the West Virginia University MIX and ENEWS systems that distribute information to students, faculty, and staff of West Virginia University. For mothers recruited nationally, a recruitment message was sent through a study recruitment site (http://www.findparticipants.com). The site pre-screens interested research participants and allows a researcher to send recruitment messages to their bank of potential participants based on specific demographic criteria. The message was targeted towards women in the United States.

Mothers who were interested in participating in the study with their child either contacted the principal investigator to begin the study or directly went to the study website. Both mothers and children were informants in the study and each mother-child pair was given a $\$ 25.00$ Target gift card for their participation. Once mothers consented to participate for themselves and their children, they were given three options to complete the questionnaires: online, over the phone, or on paper via mail. In addition, mothers and children were given the option to complete the questionnaires either at home or in the location of their choice (e.g., local library). Nearly all of the mothers completed the surveys online (123/126 pairs, 36 mothers). The remaining three pairs completed the surveys on paper via mail. Mothers completed a battery of surveys. The surveys 
included a demographic questionnaire, the Emotion Regulation Checklist (ERC; Shields \& Cicchetti, 1995) and an attributions questionnaire based on a measure used by Coplan and colleagues (2002) designed to elicit mothers' attributions regarding children's dysregulated emotion displays. Children completed a separate battery of surveys, including the Emotion Regulation Questionnaire - For Children (ERQ - C; Gullone \& Taffe, 2012), the Regulatory Emotional Self-Efficacy Scale - Adapted (RESE; Caprara \& Gerbino, 2001; Rasmussen, 2015), and the emotion socialization sub scale of the Emotions as a Child Inventory (EAC; Magai, 1996; O’Neal \& Magai, 2005).

The final sample was primarily Caucasian (84\%), and $4 \%$ of the sample were American Indian, $4 \%$ were Asian, $5 \%$ were Black or African American, and 3\% were bi- or multi-racial. The sample consisted of $85 \%$ participants were not Hispanic or Latino (11\% Hispanic or Latino, $4 \%$ did not respond). The majority of mothers in the sample were also highly educated ( $20 \%$ some college; $45 \%$ university degree; $7 \%$ some graduate school, $28 \%$ graduate degree). Most of the mothers in the sample were employed full time $(60 \%, 19 \%$ employed part-time; $15 \%$ not employed outside of the home), and the largest portion of mothers reported high family incomes (19\% income in the $\$ 100,000$ - $\$ 149,000$ range). The majority of the participants (38\%) were from the South, Division 5 (South Atlantic) U. S. Census region (Delaware, District of Columbia, Virginia, West Virginia, North Carolina, South Carolina, Georgia, Florida). The second largest portion of participants were located in the West, Division 8 (Mountain) region (Idaho, Montana, Wyoming, Nevada, Utah, Colorado, Arizona, New Mexico). Mean maternal age was 38.74 years $(S D=6.42$, range 23-63 years). Mean child age was 10.72 years $(S D=.90$, range $9-13$ years). 


\section{Mother-Report Measures}

Demographic questionnaire. Mothers completed a brief demographic questionnaire (see Appendix 1). The survey included questions about mothers' age, race and ethnicity, and mothers' educational level, occupation status, income, and current census location. Mothers also reported on their child's age, gender, and race. In addition, mothers also identified their relation to the child (e.g., biological mother, stepmother), their child's current and last grade completed, and the type of school the child was attending (e.g., public, private, home school).

Emotion Regulation Checklist. Mothers completed the Emotion Regulation Checklist (ERC; Shields \& Cicchetti, 1995; see Appendix 2). This 24-item parent-report measure was composed of two subscales assessing children's negative emotionality/negativity and (2) emotion regulation (reflective of healthy regulatory behaviors). This measure has been used with samples of children ranging from six to twelve years of age (Shields \& Cicchetti, 1995). For the purposes of this investigation, the 15-item negative emotionality/negativity subscale was used as a proxy for negative emotionality. Mothers reported on a scale of 1 (never) to 4 (almost always) how often each statement applied to their child. Sample items from the negative emotionality subscale included "Exhibits wide mood swings (child's emotional state is difficult to anticipate because he/she moves quickly from positive to negative moods)" and "Is easily frustrated." The alpha coefficient for this subscale was adequate $(\alpha=.83)$.

Temperament in Middle Childhood Questionnaire. To assess children's soothability, attention focusing, and inhibitory control, three subscales from the Temperament in Middle Childhood questionnaire were answered by mothers (Simonds \& Rothbart, 2004). The soothability subscale was eight items and included items such as "Has a hard time settling down after an exciting activity," "Is very difficult to soothe when s/he has become upset," and "When 
$\mathrm{s} /$ he cries, tends to cry for more than a couple of minutes at a time" $(\alpha=.81)$. The attentional focus subscale has seven items and included items such as "Is easily distracted when listening to a story," "Looks around the room when doing homework," and "When working on an activity, has a hard time keeping her/his mind on it" $(\alpha=.94)$. The final temperament subscale, inhibitory control, was eight items and included items such as "Can stop her/himself when s/he was told to stop," "Can stop her/himself from doing things too quickly," and "Likes to plan carefully before doing something” $(\alpha=.69)$.

Maternal attributions of dysregulated negative emotion expressions. To measure maternal attributions of children's negative emotion displays, a questionnaire developed by Coplan and colleagues (2002) was administered to mothers (see Appendix 3). Based on the negative emotions used in the Emotions as a Child Inventory (i.e., sadness, anger, fear, shame/embarrassment; Magai, 1996; O’Neal \& Magai, 2005), mothers were presented with a set of statements corresponding to each negative emotion. On a scale of 1 (not at all) to 5 (very), mothers reported the degree to which the dysregulated emotion display was (1) stable ("My child will keep acting this way" to "A stage that will pass"), (2) typical of the child ("This is just like how my child behaves" to "My child never acts this way"), (3) intentional ("My child did this on purpose" to "My child did not mean to do this" to), and (4) dispositional ("Due to my child's personality" to "Due to the situation"). For each negative emotion, all four responses were added together and divided by the number of items for each prime (i.e., four items), creating a single aggregate for maternal attributions for each negative emotion. Higher scores on the variable reflected external attributions (i.e., children's behavior is unstable, atypical, unintentional, and due to the situation) and lower scores reflected internal attributions (i.e., children's behavior is stable, typical, intentional, and due to disposition). Alpha coefficients for attributions of sadness 
( $\alpha=.77)$, anger $(\alpha=.85)$, fear $(\alpha=.87)$, and shame/embarrassment $(\alpha=.85)$ were adequate. For attributions of sadness, removal of a single item ("how much my child had control over their dysregulated display of sadness") would have improved the alpha coefficient to $\alpha=.80$.

However, for parsimony of across the four attribution variables, the item was kept, resulting in a slightly lower alpha for attributions of sadness $(\alpha=.77$ versus $\alpha=.80)$.

\section{Child-Report Measures}

In addition to mothers, children were also informants for the study. The majority of studies in the emotion socialization literature utilize only parental perspectives on emotion socialization, with a few notable exceptions (e.g., Klimes-Dougan et al., 2007). Previous research demonstrates children themselves provide a distinct perspective from parental reports of parenting behaviors (Sessa, Avenevoli, Steinberg, \& Morris, 2001). In addition, child reports of parenting may actually be more reliable than parent report, particularly when they are reporting on more negative or maladaptive parenting behaviors (Sessa et al., 2001). This is important to note given some of the emotion socialization items children responded to were in reference to nonsupportive parenting behaviors (e.g., punishing the child for expressing a negative emotion, or shutting down the emotion expression). In addition, the developing cognitive skills of older children in this sample would help children be effective informants of their emotion regulation strategies and regulatory emotional self-efficacy (e.g., metacognition, Miller et al., 2003; improved long-term memory, Schneider, 2002). Taken together, it is likely children have unique and distinct perceptions of both the parent-child relationship and their own regulatory strategies and behaviors.

\section{Emotion Regulation Questionnaire - For Children (ERQ - C; Gullone \& Taffe,}

2012). The Emotion Regulation Questionnaire - For Children (ERQ - C) is a 10-item instrument 
that assessed children's use of cognitive reappraisal and expressive suppression based upon the Emotion Regulation Questionnaire (ERQ; Gross \& John, 2003; see Appendix 4). Gullone and Taffe (2012) made slight wording changes to simplify the language of the original adult ERQ and decreased the scale anchors to a five-point scale for ease of children completing it. Children rated each item on a scale of scale of 1 (strongly disagree) to 5 (strongly agree) how much they agreed with a given emotion regulation strategy. Cognitive reappraisal is an adaptive internal strategy a child uses to change the way they think to reduce distress or to feel happier (Gullone \& Taffe, 2012). The cognitive reappraisal scale is composed of six items, such as "When I want to feel happier, I think about something different" and "When I want to feel less bad (e.g., sad, angry, or worried), I think about something different" $(\alpha=.81)$. Expressive suppression is a maladaptive internal strategy aimed at shutting down a distressing or positive emotional experience (Gullone \& Taffe, 2012). The expressive suppression scale was composed of four items, including "I control my feelings by not showing them" and "When I'm feeling bad (e.g., sad, angry, or worried), I'm careful not to show it" $(\alpha=.76)$. For cognitive reappraisal and expressive suppression scales, all items were summed together and divided by the number of items in each respective subscale to create a mean score.

\section{Regulatory Emotional Self-Efficacy Scale - Adapted (RESE - Adapted; Caprara \&}

Gerbino, 2001; Rasmussen, 2015). To assess children's self-efficacy beliefs regarding their ability to regulate their negative emotions, the Regulatory Emotional Self-Efficacy Scale Adapted (RESE - Adapted; Caprara \& Gerbino, 2001; Rasmussen, 2015; see Appendix 5) was administered. The original Caprara and Gerbino (2001) measure has been used with adolescents (Bandura et al., 2003) and with emerging adults (Caprara et al., 2008). For use with older children, the scale was adapted by Rasmussen (2015). The wording of the questions was changed 
to be more developmentally appropriate for children. This 12 -item self-report measure was composed of three main subscales, perceived self-efficacy in managing positive affect, perceived self-efficacy in managing despondency (i.e., sadness), and perceived self-efficacy managing anger. For the purposes of this investigation, the perceived self-efficacy in despondency and anger were combined to create a single aggregate of perceived self-efficacy managing negative emotions $(\alpha=.86)$. The aggregate was composed of eight items. Sample items included "How well can you avoid flying off the handle when you get angry?", "How good are you at getting over being annoyed quickly for unfair things you have experienced?", and "How good are you at keeping yourself from feeling discouraged when someone criticizes you strongly?" Children responded on a scale of 1 (not good) to 5 (very good) scale how well they could execute each regulatory strategy or behavior. For the self-efficacy to regulate negative emotions aggregate, all items were summed together and divided by the number of items in the subscale to create a mean score.

\section{Emotions as a Child Inventory (EAC; Magai, 1996; O’Neal \& Magai, 2005).}

Children completed the emotion socialization subscale of the Emotions as a Child Inventory (EAC; Magai, 1996; O’Neal \& Magai, 2005; see Appendix 6). Children were asked to think about at times when they felt angry (anger), sad (sadness), afraid (fear), or embarrassed (shame/embarrassment). For each emotion, children responded to a set of statements describing emotion socialization responses corresponding to the O'Neal and Magai (2005) configuration of emotion socialization (i.e., neglect, override, magnify, reward, punish) and were asked to report how much it is like their mother to use one of the seven emotion socialization responses described above. For each statement, children reported on a scale of 1 (not at all like my mother) to 7 (exactly like my mother) how much the emotion socialization practice was like their mother. 
There is some inconsistency in the developmental literature regarding the factor structure of the Emotions as a Child Inventory and which of the five emotion socialization strategies (i.e., neglect, override, magnify, reward, punish) constitute supportive and nonsupportive responses, and how the structure might vary which specific negative emotion is being tested (e.g., Guo, Mrug, \& Knight, 2016; Klimes-Dougan et al., 2007; O’Neal \& Magai, 2005). As a result, a series of exploratory factor analyses (EFAs) were run to examine the factor structure of maternal emotion socialization practices for each of the four specific emotions, sadness, anger, fear, and shame/embarrassment (see Table 7). Factor analyses were run using varimax rotation, and the final solution included rotated factor loadings. Factor loadings of .4 and above were included in their respective factors (Hinkin, 1998; Howard, 2016). In situations where a single item loaded on more than one factor, the highest loading of the two was kept within its factor, and the lower loading was eliminated from its factor.

The final factor structures for each emotion yielded more than a simple supportive and nonsupportive factor structure for each emotion, and the number of factors varied across the specific emotions (sadness $=5$ factors, $61.52 \%$ of variance; anger, 4 factors, $62.84 \%$ of variance; fear, 4 factors; $59.1 \%$ of variance; shame/embarrassment, 3 factors, $59.62 \%$ of variance). In total, across the four specific emotions, five types of supportive and six types of nonsupportive emotion socialization were found.

For the purposes of this study, the supportive - emotionally scaffolding/comforting and nonsupportive - punitive/neglecting factors for each of the four emotions were used, and remaining factors were not included. The supportive-emotionally scaffolding/comforting factor was defined as maternal behavior that actively understood and helped the child understand the emotion they were feeling and behavior that actively engaged in specific comforting behaviors 
(e.g., holding the child, telling the child not to be afraid). The nonsupportive punitive/neglecting factor was defined as engagement in punitive or insensitive behaviors in response to the child's expression of the specific emotion, (e.g., putting the child down for feeling the emotion, telling the child they are foolish for expressing the emotion), or simply ignoring the child's emotional display altogether. Based on the factor analyses, final supportive emotionally scaffolding/comforting and nonsupportive - punitive/neglecting subscales were created for each of the four emotions and will be referred hereafter as supportive and nonsupportive, and these were used for analyses. The alpha coefficients for each emotion for supportive and nonsupportive responses were adequate ( $\alpha$ s ranging from .70 - .82).

\section{Data Analysis Plan}

Data were analyzed using multiple regression analysis and a bootstrapping technique to test for indirect effects based on Preacher and Hayes (2004; see Table 8). For all three of the research questions, preliminary demographic analyses were run to assess which maternal and child control variables should be included in the regression models, including dummy-coded child sex $(0=$ male $; 1=$ female $)$, child age, maternal race, maternal education, maternal occupation status, and family income. For the first and third research questions, control variables were entered in the first block, main effects were entered in the second block, and any interaction term (s) were entered in the third block. All predictor variables in the regression analyses were centered prior to computing interaction terms and these values were used in the regression models.

Research question one. The first research question examined the relation between children's negative emotionality, soothability, attention focusing, and inhibitory control and maternal attributions of specific negative emotion displays. Standard multiple regression was 
used to examine the relation between four dimensions of temperament (i.e., negative emotionality, soothability, attention-focusing, and inhibitory control), and maternal attributions of children's dysregulated displays of sadness, anger, fear, and shame/embarrassment. Given maternal attributions were measured as a single continuous variable for each emotion, a single regression model was run for each specific emotion, totaling four regression models.

\section{Diagnostic analyses and assessment of assumptions - research question one.}

Diagnostic analyses for the regression models for research question 1 are included in Table 1 per guidelines by Field (2013). These analyses indicated the Cook's distance values were underneath one and the Malahalanobis distances were all below 25, indicating that existing outliers did not influence the overall regression model (Cook \& Weisberg, 1982; Field, 2013). In addition, examination of scatterplots with studentized and standardized residuals plotted against standardized predictor values for all four maternal attribution models (i.e., sadness, anger, fear, shame/embarrassment) indicated there were slight patterns, but the assumption of homoscedasticity was generally met. In addition, while some of the temperamental predictors were correlated with one another strongly (e.g., child negative emotionality and soothability, $r=$ $.72, p<.001$ ), none of the correlations were beyond .8 , and the variance inflation factor (VIF) values for the predictors across all models were less than 10 (Bowerman \& O'Connell, 1990).

Research question two. The second research question tested whether child temperament (i.e., negative emotionality, soothability, attention focusing, inhibitory control) was indirectly related to maternal emotion socialization via maternal attributions of children's dysregulated emotion displays (i.e., sadness, anger, fear, shame/embarrassment). A bootstrapping method described by Preacher and Hayes (2004) and Mathieu and Taylor (2006) was used to test the indirect effect of maternal attributions of specific emotion displays on the association between 
the four components of children's temperament and maternal supportive and nonsupportive emotion socialization responses to sadness, anger, fear, and shame/embarrassment. This method was selected because the sample size of the proposed investigation was small $(n<400)$. As a result, the Sobel test lacked statistical power due to the skewed distribution of the effects of the independent variable on the indirect effect and indirect effect and the dependent variable (Dearing \& Lawrence, 2006; Hayes, 2009). A bootstrapping approach was prudent given it corrects for the bias of a skewed distribution resulting from a small sample size (i.e., $n<400$ ). This takes place by drawing a specified number of artificial samples of a particular sample size of $n$, using the original sample (Dearing \& Lawrence, 2006). This method yields a bootstrapped estimate of the indirect effect of the mediating variable on the relation between the independent and dependent variable using 95\% and 99\% confidence intervals (Preacher \& Hayes, 2004). So long as zero is not within the confidence interval, it is concluded the indirect effect of the variable is significantly different from zero (Dearing \& Lawrence, 2006; Preacher \& Hayes, 2004). No diagnostic analyses or assessment of assumptions were included for research question two because bootstrapping was the main analysis used instead of regression. Discussion of the normality of child temperament, maternal attributions of specific emotion displays and emotion socialization responses to sadness, anger, fear, and shame/embarrassment are discussed below.

Research question three. The third research question examined if maternal supportive and nonsupportive emotion socialization responses to specific emotions moderated the relations between children's temperament (i.e., negative emotionality, soothability, attention focusing, inhibitory control) and children's cognitive reappraisal, expressive suppression, and regulatory emotional self-efficacy. Multiple regression analyses were conducted using procedures prescribed by Cohen, Cohen, West, and Aiken (2003) to examine the moderating influence of 
maternal supportive and nonsupportive emotion socialization responses to specific emotions on the relation between child temperament and emotion-related outcomes, namely cognitive reappraisal, expressive suppression, and regulatory emotional self-efficacy. For moderation to be achieved, the interaction between the independent variable (i.e., negative emotionality, soothability, attentional focusing, inhibitory control) and the moderator (supportive and nonsupportive maternal emotion socialization responses to specific emotions) must be significant (Dearing \& Lawrence, 2006). If significant, the interaction will be probed, and the component of temperament and the significant child outcome will be plotted at low (- $1 S D)$, medium (mean), and high $(+1 S D)$ levels of the significant emotion socialization type (i.e., supportive or nonsupportive maternal emotion socialization; Cohen et al., 2003).

\section{Diagnostic analyses and assessment of assumptions - research question three.}

Diagnostic analyses and assessment of assumptions for the regression models for research question 3 are included in Tables 2-4 per guidelines by Field (2013). For cognitive reappraisal, all of the Cook's distance values were underneath 1, but only two of the Mahalanobis distances values were underneath 25 (cognitive reappraisal in relation to fear and shame/embarrassment; see Table 2). The remaining two Mahalanobis distances for cognitive reappraisal in relation to sadness and anger were slightly above the cutoff of 25 (Cook \& Weisberg, 1982; see Table 2). In addition, examination of scatterplots with studentized and standardized residuals plotted against standardized predictor values for all four models (i.e., cognitive reappraisal in relation to sadness, anger, fear, shame/embarrassment) indicated there were slight patterns, but the assumption of homoscedasticity was generally met. In addition, as discussed with research question one, while some of the temperamental predictors were correlated with one another strongly (e.g., child negative emotionality and soothability, $r=.72, p<.001$ ), none of the 
correlations were beyond .8 , and the variance inflation factor (VIF) values for the predictors across all models were less than 10 (Bowerman \& O'Connell, 1990).

For expressive suppression, all of the Cook's distance values were underneath one, but only one of the four models had a Malahobis distances underneath 25 (i.e., expressive suppression in relation to emotion socialization of sadness; see Table 3). For the remaining three models (i.e., expressive suppression in relation to anger, fear, and shame/embarrassment), the Mahalanobis distances values were quite large and above the cutoff of $25(72.69,54.02,68.84$ for anger, fear, and shame/embarrassment, respectively; Barnett \& Lewis, 1978). This suggests the existing outliers may have significantly influenced the regression models for expressive suppression. In addition, examination of scatterplots with studentized and standardized residuals plotted against standardized predictor values for all four models (i.e., expressive suppression in relation to emotion socialization of sadness, anger, fear, shame/embarrassment) indicated there were slight patterns, but the assumption of homoscedasticity was generally met. In addition, as noted above, while some of the temperamental predictors were correlated with one another strongly (e.g., child negative emotionality and soothability, $r=.72, p<.001$ ), none of the correlations were beyond .8, and the variance inflation factor (VIF) values for the predictors across the expressive suppression models were less than 10 (Bowerman \& O’Connell, 1990).

For regulatory emotional self-efficacy, all of the Cook's distance values were underneath the cutoff of one, and as with expressive suppression, only one of the models (regulatory emotional self-efficacy in relation to emotion socialization of sadness (see Table 4) was underneath the Mahalanobis distances cutoff of 25, and the remaining three models (i.e., regulatory emotional self-efficacy in relation to emotion socialization of anger, fear, and shame/embarrassment) had quite large values $(28.88,60.86,63.34$ for anger, fear, and 
shame/embarrassment, respectively; Barnett \& Lewis, 1978). This suggests outliers may have influenced the final regression models for regulatory emotional self-efficacy. In addition, examination of scatterplots with studentized and standardized residuals plotted against standardized predictor values for all four models (i.e., regulatory emotional self-efficacy in relation to emotion socialization of sadness, anger, fear, shame/embarrassment) indicated there were slight patterns, but the assumption of homoscedasticity was generally met. In addition, while some of the temperamental predictors were correlated with one another strongly (e.g., child negative emotionality and soothability, $r=.72, p<.001$ ), none of the correlations were beyond .8 , and the variance inflation factor (VIF) values for the predictors across all models were less than 10 (Bowerman \& O’Connell, 1990).

Normality of variables. Histograms of the four temperament variables, maternal attributions of sadness, anger, fear, and shame/embarrassment, the emotion socialization variables (i.e., supportive and nonsupportive responses to sadness, anger, fear, and shame/embarrassment), and the three emotion outcomes (i.e., cognitive reappraisal, expressive suppression were examined and compared to the standard normal curve.

Child negative emotionality was very slightly positively skewed and somewhat leptokurtic. Soothability, attention focusing, and inhibitory control were all slightly negatively skewed and soothability and inhibitory control were somewhat leptokurtic. For the variables for maternal attributions of sadness, anger, and fear, the distributions were slightly negatively skewed and somewhat leptokurtic. For the maternal emotion socialization variables across all emotions (i.e., supportive and nonsupportive responses to sadness, anger, fear, and shame/embarrassment), nonsupportive responses to each specific emotion were quite positively skewed (i.e., the majority of responses were one to two standard deviations below the mean) and 
supportive responses were quite negatively skewed (i.e., the majority of responses were one to two standard deviations above the mean). All variables were somewhat leptokurtic. For the three emotion outcomes, were somewhat differentiated from one another. The distribution for cognitive reappraisal was slightly negatively skewed, as was the distribution for regulatory emotional self-efficacy. The distribution for expressive suppression generally followed the normal curve, with some exceptions above and below the mean.

\section{Statistical Power Analyses}

A priori statistical power analyses were run using GPower software (version 3.1.9.2) to ascertain the appropriate sample size needed to detect a medium-sized effect size in a multiple regression $f$-test $(\mathrm{ES}=.15$, Cohen, 1992). With an $\alpha=.05$ and statistical power set at .95 , the projected sample size needed to detect a medium-sized effect was approximately 120 participants. As a result, the sample size of this study (i.e., 123 mother-child pairs and 36 mothers) was adequate to meet the objectives of this investigation. 


\section{Chapter 4}

\section{Results}

Descriptive statistics for the variables of interest in the study are presented in Table 5. In addition, correlations between study variables are presented in Table 6. Any results with $p$ values between .051 and .08 are denoted in subsequent tables as trend-level findings. Negative emotionality was significantly, negatively related to a lower level of maternal attributions (i.e., indicative of internal attributions) for all four specific negative emotions, and soothability, attention focusing, and inhibitory control were all significantly, positively related to maternal attributions (i.e., responses indicative of external attributions) of all four specific emotions.

In terms of maternal emotion socialization responses to specific emotions, negative emotionality was significantly, positively related to nonsupportive responses to sadness, anger, fear, and shame/embarrassment (see Table 6). Negative emotionality was significantly, negatively related to supportive responses to sadness, anger, fear, and shame/embarrassment. For soothability, the opposite pattern was true; soothability was negatively related to nonsupportive responses to specific emotions, and was positively related to supportive responses to sadness, anger, fear, and shame/embarrassment. In addition, for attention focusing, high levels of attention focusing were significantly and negatively related to lower levels of nonsupportive responses to sadness, anger, fear, and shame/embarrassment. High levels of attention focusing were also significantly and positively related to supportive responses to sadness. For inhibitory control, high levels of inhibitory control were significantly and negatively related to nonsupportive responses to fear and shame, and significantly and positively related to supportive responses to sadness and anger. 


\section{Research Question One: Child Temperament Predicting Maternal Attributions of Specific Emotion Displays}

Preliminary regression models were run to control for demographic covariates in predicting maternal attributions of dysregulated displays of sadness, anger, fear, and shame/embarrassment. These demographic variables included child age, child gender, maternal race, maternal education, maternal occupational status, and family income. In the four models predicting maternal attributions of sadness, anger fear, and shame/embarrassment, none of these control variables were significantly related to maternal attributions of expression of specific emotions (see Table 8).

Maternal attributions of sadness. The final regression model for maternal attributions of sadness was significant, Adjusted $R^{2}=.24, F(4,139)=12.52, p<.001$, (see Table 9). Child soothability was significantly and positively related to maternal attributions of sadness $(ß=.34$, $p=.003$ ); a high level of child soothability was related to a high level of maternal attributions of sadness (i.e., responses indicating more external attributions). Negative emotionality, attention focusing, and inhibitory control were not significantly related to maternal attributions of sadness.

Maternal attributions of anger. The final model for maternal attributions of anger was significant, Adjusted $R^{2}=.32, F(4,139)=18.03, p<.001$ (see Table 9). Negative emotionality and attention focusing were significantly negatively related to maternal attributions of anger (negative emotionality, $\beta=-.31, p=.014$; attention focusing, $\beta=-.27, p=.018$ ); thus, higher negative emotionality and attentional focusing were associated with lower levels of maternal attributions (i.e., responses indicating more internal attributions). In addition, child soothability and inhibitory control were significantly and positively related to maternal attributions of anger (soothability, $\beta=.40, p<.001$; inhibitory control, $\beta=.19, p=.042$ ); high levels of child 
soothability and inhibitory control were related to a high level of maternal attributions of anger (i.e., indicative of external attributions).

Maternal attributions of fear. The final model for maternal attributions of fear was significant, Adjusted $R^{2}=.29, F(4,136)=12.27, p<.001$, (see Table 9). Child soothability and inhibitory control were significantly and positively related to maternal attributions of fear (soothability, $\beta=.42, p<.001$; inhibitory control, $\beta=.21, p=.024$ ); high levels of child soothability and inhibitory control were related to a high level of maternal attributions of fear (i.e., responses indicating more external attributions). Negative emotionality and attention focusing were not significantly related to maternal attributions of fear.

Maternal attributions of shame/embarrassment. The final regression model for maternal attributions of shame/embarrassment was significant, Adjusted $R^{2}=.20, F(4,137)=$ 9.80, $p<.001$, (see Table 9). Child soothability and inhibitory control were positively and significantly related to maternal attributions of shame/embarrassment (soothability, $\beta=.33, p=$ .005 ; inhibitory control, $\beta=.21, p=.038$ ); high levels of child soothability and inhibitory control were related to a high level of maternal attributions of shame/embarrassment (i.e., responses indicating external attributions). Negative emotionality and attention focusing were not significantly related to maternal attributions of shame/embarrassment.

\section{Research Question Two: Indirect Effects of Child Temperament on Maternal Emotion Socialization of Specific Emotions via Maternal Attributions of Specific Emotions}

The PROCESS macro (Darlington \& Hayes, 2017) was used to test for indirect effects of child temperament on maternal emotion socialization via maternal attributions of children's dysregulated displays of sadness, anger, fear, and shame/embarrassment. The number of bootstrap samples drawn from the original sample of 123 mother-child pairs for bias-corrected 
bootstrap confidence intervals was 5000, based on recommendations by Hayes (2009). In these analyses, the presence of an indirect effect assumes a significant relation between the particular temperamental dimension and maternal attributions (the $\mathrm{x}>\mathrm{m}$ path) and maternal attributions and maternal emotion socialization (the $\mathrm{m}>\mathrm{y}$ path; Mathieu \& Taylor, 2006). In addition, as with research question 1 , demographic variables were preliminarily tested as covariates in the relation between child temperament and maternal supportive and nonsupportive emotion socialization of sadness, anger, fear, and shame/embarrassment (see Table 10). Any significant control variables were included in the final regression analyses. Note the PROCESS macro simultaneously conducts multiple regression analyses for the variables of interest at the same time as the bootstrapping; findings and beta weights for these regression models can be found in Tables 13 through 20.

Maternal attributions of sadness. Regression analyses were used to examine the indirect effect of child temperament on maternal supportive and nonsupportive emotion socialization responses to sadness via maternal attributions of sadness. Per an examination of the preliminary demographic models, family income was included as a control in the models for nonsupportive emotion socialization responses to sadness (see Table 10).

Negative emotionality. Child negative emotionality did not indirectly affect maternal supportive emotion socialization responses to sadness via maternal attributions of sadness, but did indirectly affect nonsupportive emotion socialization responses (see Table 12). In addition, there was also a slight but significant effect of family income $(\beta=.04, p=.02$; a high level of family income was related to a high level of nonsupportive emotion socialization.

Soothability. Child soothability did not indirectly affect maternal supportive responses to sadness via maternal attributions of sadness, but did indirectly affect nonsupportive responses 
(see Table 12). There was a significant effect for family income $(\beta=.05, p=.006)$; high family income was related to a high level of nonsupportive emotion socialization.

Attention focusing. Attention focusing did not indirectly affect maternal supportive emotion socialization responses via maternal attributions of sadness, but did indirectly affect nonsupportive responses (see Table 12). In addition, for nonsupportive emotion socialization, there was a significant indirect effect of maternal attributions of sadness and a significant effect of family income $(ß=.05, p=.011)$; a high level of family income was related to a high level of nonsupportive emotion socialization.

Inhibitory control. Inhibitory control did not indirectly affect maternal supportive responses to sadness via maternal attributions of sadness, but did indirectly affect nonsupportive responses (see Table 12). In addition, there was a significant effect for family income $(ß=.04 ; p$ $=.016)$; a high family income was related to a high level of nonsupportive emotion socialization.

Maternal attributions of anger. Regression analyses were used to examine the indirect effect of child temperament on maternal supportive and nonsupportive emotion socialization responses to anger via maternal attributions of anger. Child age was used as a control for supportive emotion socialization to anger (see Table 11).

Negative emotionality. Child negative emotionality indirectly affected both maternal supportive and nonsupportive emotion socialization responses via maternal attributions of anger, (see Table 12). There was a significant effect for child age $(\beta=-.41 ; p<.001)$; older child age was associated with a low level of maternal supportive emotion socialization responses to anger. For nonsupportive responses, there was a significant indirect effect for maternal attributions of anger. 
Soothability. Child soothability did not indirectly affect maternal supportive responses to anger via maternal attributions of anger but did indirectly affect nonsupportive responses (see Table 12). In addition, there was a significant effect for child age; older child age was related to a low level of supportive emotion socialization responses to anger $(ß=-.31 ; p=.006)$. There was not a significant indirect effect of maternal attributions of anger on the relation between soothability and nonsupportive emotion socialization responses to anger.

Attention focusing. Attention focusing indirectly affected maternal supportive emotion socialization responses via maternal attributions of anger (see Table 12). In addition, there was a significant effect of child age on supportive responses to anger $(ß=-.35, p=.003)$; older child age was related to a low level of supportive emotion socialization responses to anger. Attention focusing also indirectly affected maternal nonsupportive responses to anger via maternal attributions of anger.

Inhibitory control. Inhibitory control did not indirectly affect maternal supportive responses to sadness via maternal attributions of sadness, but did indirectly affect nonsupportive responses (see Table 12).

Maternal attributions of fear. Regression analyses were used to examine the indirect effect of child temperament on maternal supportive and nonsupportive emotion socialization responses to fear via maternal attributions of fear. Per the preliminary demographic models, there were no significant controls included in these models (see Tables 10 and 11).

Negative emotionality. Child negative emotionality did not indirectly affect either maternal supportive or nonsupportive emotion socialization responses to fear via maternal attributions of fear (see Table 12). 
Soothability. Child soothability did not indirectly affect either maternal supportive or nonsupportive emotion socialization responses to fear via maternal attributions of fear (see Table 12).

Attention focusing. Child attention focusing did not indirectly affect maternal supportive responses to fear via maternal attributions of fear, but did indirectly affect nonsupportive responses to fear (see Table 12).

Inhibitory control. Child inhibitory control did not indirectly affect maternal supportive responses to fear via maternal attributions of fear, but did indirectly affect nonsupportive responses to fear (see Table 12).

Maternal attributions of shame/embarrassment. Regression analyses were used to examine the indirect effect of child temperament on maternal supportive and nonsupportive emotion socialization responses to shame/embarrassment via maternal attributions of shame/embarrassment. Per the preliminary demographic models, there were no significant control included in these models (see Tables 10 and 11).

Negative emotionality. Child negative emotionality did not indirectly affect maternal supportive emotion socialization responses to shame/embarrassment via maternal attributions of shame/embarrassment, but did indirectly affect nonsupportive emotion socialization responses (see Table 12).

Soothability. Child soothability did not indirectly affect maternal supportive responses to shame/embarrassment via maternal attributions of shame/embarrassment, but did indirectly affect nonsupportive responses to fear (see Table 12). 
Attention focusing. Attention focusing did not indirectly affect maternal supportive emotion socialization responses to shame/embarrassment via maternal attributions of shame/embarrassment, but did indirectly affect nonsupportive responses to it (see Table 12).

Inhibitory control. Inhibitory control did not indirectly affect maternal supportive responses to sadness via maternal attributions of sadness, but did indirectly affect nonsupportive responses (see Table 12).

\section{Research Question Three: Maternal Emotion Socialization as Moderator Between Child}

\section{Temperament and Emotion Outcomes}

As with the research questions 1 and 2, demographic variables were tested as covariates in the relation between child temperament and the three emotion outcomes, cognitive reappraisal, expressive suppression, and regulatory emotional self-efficacy. Any significant control variables were included in the final regression analyses (see Table 21). Per these analyses, child sex was included as a control for child expressive suppression. In addition, given the high potential volume of interaction terms that could be tested for this research question (4 temperament indicators X 4 specific emotions X 2 types of maternal emotion socialization $=32$ possible interaction terms), a correlational analysis was run to assess the relations between all the possible interaction terms and the three child emotion outcomes. Any significant interaction terms were included in the final models for each of the respective emotions.

Cognitive reappraisal. Per the preliminary demographic analyses, no control variables were included for any of the regulatory emotional self-efficacy models (see Table 21). In addition, no interaction terms were included for cognitive reappraisal given none were significant in the correlation analysis noted above. 
Sadness. The final regression model for cognitive reappraisal in relation to emotion socialization responses to sadness was significant, Adjusted $R^{2}=.11, F(6,106)=3.38, p=.004$ (see Table 22). Inhibitory control was significantly positively related to cognitive reappraisal ( $(3$ $=.40, p=.005)$; a high level of inhibitory control was related to a high level of cognitive reappraisal. The three remaining temperament dimensions, negative emotionality, soothability, and attention focusing, and the two maternal emotion socialization types were not significantly related to cognitive reappraisal.

Anger. The final regression model for cognitive reappraisal in relation to emotion socialization responses to anger was significant, Adjusted $R^{2}=.16, F(6,109)=4.72, p<.001$, (see Table 22). Inhibitory control and supportive maternal emotion socialization responses to anger were significantly positively related to cognitive reappraisal (inhibitory control, $(ß=.33, p$ $=.017$; supportive emotion socialization, $(\beta=.26, p=.007)$; a high level of inhibitory control and supportive maternal emotion socialization to anger were related to a high level of cognitive reappraisal. The three remaining temperament indicators and nonsupportive maternal emotion socialization were not significantly related to cognitive reappraisal.

Fear. The final regression model for cognitive reappraisal in relation to emotion socialization responses to fear was significant, Adjusted $R^{2}=.15, F(6,105)=4.20, p=.001$ (see Table 22). The same pattern displayed regarding cognitive appraisal in relation to the emotion socialization responses to anger was present in relation to fear. Inhibitory control and supportive maternal emotion socialization responses to fear were significantly positively related to cognitive reappraisal (inhibitory control, $(\beta=.36, p=.011$; supportive emotion socialization, $(\beta=.23, p=$ .017); a high level of inhibitory control and supportive maternal emotion socialization were 
related to a high level of cognitive reappraisal. The three remaining temperament indicators and nonsupportive maternal emotion socialization responses were not significant.

Shame/embarrassment. The final model for cognitive reappraisal in relation to maternal emotion socialization responses to shame/embarrassment was significant, Adjusted $R^{2}=.13$, $F(6,107)=3.86, p=.002$ (see Table 122 ). Inhibitory control was the only variable significantly related to cognitive reappraisal $(\beta=.33, p=.024)$; a high level of inhibitory control was related to a high level of cognitive reappraisal.

Expressive suppression. Per the preliminary demographic analyses, child sex was included as a control for expressive suppression (see Table 21).

Sadness. The final model for expressive suppression in relation to maternal emotion socialization responses to sadness was significant, Adjusted $R^{2}=.19, F(7,103)=4.62, p<.001$ (see Table 23). None of the four temperament indicators were significantly related to expressive suppression. Child sex and supportive maternal emotion socialization to sadness were significantly negatively related to expressive suppression (child sex, $\beta=-.19, p=.035$; supportive emotion socialization, $\beta=-.32, p=.002$ ); female sex and a high level of supportive maternal emotion socialization in response to sadness were related to a low level of expressive suppression.

Anger. The final model for expressive suppression in relation to maternal emotion socialization responses to anger was significant, Adjusted $R^{2}=.17, F(9,104)=3.63, p=.001$ (see Table 23). The same pattern that emerged for expressive suppression in relation to emotion socialization responses to sadness was present for anger. Child sex and supportive maternal emotion socialization in response to anger were significant predictors (child sex, $\beta=-.21, p=$ .02 ; supportive emotion socialization, $\beta=-.29, p=.002$ ); female sex and a high level of 
supportive maternal emotion socialization in response to sadness were related to a low level of expressive suppression. Neither of the interaction terms (negativeemotionalityXnonsupportivetoanger, soothabilityXnonsupportivetoanger) were significant predictors.

Fear. The final model for expressive suppression in relation to maternal emotion socialization responses to fear was significant, Adjusted $R^{2}=.18, F(10,100)=3.38, p=.001$ (see Table 23). Child gender and neither of the four temperament indicators or the three interaction terms (negativeemotionalityXnonsupportivetofear, attentionfocusingXnonsupportivetofear, inhibitorycontrolXnonsupportivetofear) were significantly related to expressive suppression. However, maternal supportive emotion socialization responses to fear were significantly and negatively related to expressive suppression $(ß=-.26, p=.007)$; a high level of supportive emotion socialization responses to fear was related to a low level of expressive suppression. Maternal nonsupportive emotion socialization responses to fear approached significance.

Shame/embarrassment. The final model for expressive suppression in relation to maternal emotion socialization responses to fear was significant, Adjusted $R^{2}=.19, F(10,101)=$ $3.68, p<.001$ (see Table 23). Child sex and inhibitory control were significantly related to expressive suppression (child sex, $\beta=-.20, p=.02$; inhibitory control; $\beta=.31, p=.031$ ); female sex was related to a low level of expressive suppression, and a high level of inhibitory control was related to a high level of expressive suppression. Supportive maternal emotion socialization responses to shame/embarrassment were also significantly negatively related to expressive suppression (supportive emotion socialization, $\beta=-.31, p=.002$ ); a high level of supportive emotion socialization responses to shame/embarrassment was related to a low level of expressive 
suppression. The three remaining temperament indicators, nonsupportive maternal emotion socialization responses to shame/embarrassment, and the three interaction terms (negativeemotionalityXnonsupportivetoshame, attentionfocusingXnonsupportivetoshame, soothabilityXnonsuportivetoshame) were not significantly related. Attention focusing approached significance.

Regulatory emotional self-efficacy. Per the preliminary demographic analyses, no control variables were included for any of the regulatory emotional self-efficacy models (see Table 21).

Sadness. The final model for regulatory emotional self-efficacy in relation to maternal emotion socialization responses to sadness was not significant (see Table 24).

Anger. The final model for regulatory emotional self-efficacy in relation to maternal emotion socialization responses to anger was significant, Adjusted $R^{2}=.10, F(6,110)=3.23, p=$ .006 (see Table 24). None of the temperament variables and nonsupportive emotion socialization responses to fear were significantly related to regulatory emotional self-efficacy. Maternal supportive emotion socialization responses to anger were the only significant variable $(ß=.32, p$ $=.001$ ); a high level of supportive emotion socialization responses to anger was related to a high level of regulatory emotional self-efficacy.

Fear. The final model for regulatory emotional self-efficacy in relation to maternal responses to anger was significant, Adjusted $R^{2}=.12, F(10,100)=2.54, p=.009$ (see Table 24). While the final model was significant, none of the four temperament indicators, supportive or nonsupportive emotion socialization responses to fear, and the interaction terms (negativeemotionalityXnonsupportivetofear, soothabilityXnonsupportivetofear, attentionfocusingXnonsupportivetofear, inhibitorycontrolXnonsupportive to fear) were 
statistically significant. The closest variable to significance was the interaction term for negativemotionalityXnonsupportivetofear $(p=.08)$.

Shame/embarrassment. The final model for regulatory emotional self-efficacy in relation to maternal emotion socialization in response to shame/embarrassment was significant, Adjusted $R^{2}=.11, F(7,106)=2.97, p=.007$ (see Table 24). In addition to supportive maternal emotion socialization responses to shame/embarrassment, none of the temperament variables were statistically significant. Nonsupportive maternal emotion socialization responses to shame/embarrassment were significantly and negatively related to regulatory emotional selfefficacy of negative emotions $(~(=-.27, p=.020)$; a high level of nonsupportive emotion socialization responses to shame/embarrassment was related to a low level of regulatory emotional self-efficacy. The interaction between negative emotionality and nonsupportive responses to shame/embarrassment was significant. The interaction was probed and modeled at low (-1 SD), medium (M), and high (+1 SD) levels of nonsupportive emotion socialization responses to shame/embarrassment (see Figure 3). At medium and high levels of nonsupportive responses to shame/embarrassment, negative emotionality was significantly and negatively related to regulatory emotional self-efficacy of negative emotions; a high level of negative emotionality was related to a low level of regulatory emotional self-efficacy. 


\section{Chapter 5}

\section{Discussion}

Both parents and children are vital contributors to the parent-child relationship and the developmental trajectories of children (Belsky, 1984; Kuczynski \& Parkin, 2007). In particular, children's temperamental traits and parental attributions of children's dysregulated negative emotion displays are important because they precede supportive and nonsupportive maternal emotion socialization responses. The aims of this study were threefold. The first aim was to examine how children's negative emotionality, soothability, attention focusing, and inhibitory control predicted maternal attributions of children's expressions of dysregulated negative emotions (i.e., sadness, anger, fear, and shame/embarrassment). The second aim was to examine whether children's temperament indirectly affected maternal supportive and nonsupportive responses to specific emotions via maternal attributions of each specific emotion. Finally, the third aim of this study was to examine whether maternal emotion socialization responses moderated the relation between children's negative emotionality, soothability, attention focusing, and inhibitory control and their cognitive reappraisal, expressive suppression, and regulatory emotional self-efficacy. Findings will be discussed in detail below, and are organized by research question. A summary of all significant findings for each research question across are included in Figures 4, 5, and 6.

\section{Research Question One: Child Temperament Predicting Maternal Attributions of Specific}

\section{Negative Emotions}

The first research question examined the relation between children's negative emotionality, soothability, attention focusing, and inhibitory control and maternal attributions of displays of sadness, anger, fear, and shame/embarrassment. For all four emotions, based on 
evidence linking children's difficultness and internal attributions of behavior (Bugental \& Shennum, 1984; Bugental et al., 1990; Gretarsson \& Gelfand, 1988), it was hypothesized that negative emotionality would be related to a low level of attributions for that particular emotion (i.e., indicative of internal attributions), and for the three remaining temperamental traits that promote emotion regulation (i.e., soothability, attention focusing, inhibitory control), these variables would be related to a high level of attributions (i.e., external attributions).

In general, the pattern of findings suggested that across emotions, at least one or more of the three emotion regulation-promoting temperamental traits (i.e., soothability, attention focusing, inhibitory control) were related to a higher level of maternal attributions, indicative of external attributions. These findings provide further evidence of the nature of parents' internal versus external attributions of negative traits or behaviors. In the parenting literature, there are two conflicting explanations of the valence of different types of parental attributions and their relation to children's traits or behaviors (Coplan et al., 2002; Miller, 1995). The first explanation posits that children's positive behaviors are seen by parents as dispositional, purposeful, and a part of who the child is (i.e., indicative of internal attributions) whereas more negative or challenging behaviors are more transitory and contextual (i.e., indicative of external attributions; Coplan et al., 2002; Goodnow, Knight, \& Cashmore, 1986; Gretarsson \& Gelfand, 1988). The second explanation posits that negative social behaviors or traits are conceptualized as more dispositional, purposeful, and related to more internal parental attributions (Dix \& Lochman, 1990; Gretarsson \& Gelfand, 1988; Miller, 1995). The findings of this study support the latter view that negative or challenging temperamental traits that undermine emotion regulation are related to parents' internal attributions of children's dysregulated emotion displays. The findings within and across specific emotions are discussed below. 
Maternal attributions of sadness. For sadness, the only significant predictor of maternal attributions of sadness was soothability; a high level of soothability was related to mothers' reports of high levels of maternal attributions of sadness (i.e., indicative of external attributions). Based on the explanation that parents hold more internal attributions of children's negative or challenging behaviors (Gretarrson \& Gelfand, 1988; Miller, 1995), it makes sense that children's ability to soothe themselves in the face of distressing stimuli would be related to more external attributions of sadness. In addition, if mothers perceive their child is highly soothable and able to effectively calm themselves, when the child is expressing dysregulated sadness, it is likely mothers would assume there was an external factor to explain why they are expressing sadness in that way (e.g., the child was tired or hungry; Root \& Rasmussen, 2017).

Maternal attributions of anger. For anger, all four temperamental traits were significant. A high level of negative emotionality and attention focusing were related to a low level of attributions (i.e., indicative of internal attributions), and the remaining two traits, soothability and inhibitory control, were related to a high level of attributions (i.e., indicative of external attributions). In the case of negative emotionality, mothers who perceived their child as being high in negative emotionality reported a low level of attributions of dysregulated displays of anger (i.e., indicative of internal attributions). There is currently little evidence demonstrating how parents respond to emotion and emotion regulation-related temperamental traits specifically. In the past, others have examined how other aspects of temperament and social behaviors impact parental attributions (e.g., aggression, Dix \& Lochman, 1990; aggression, shyness; Hastings \& Rubin, 1999; Mills \& Rubin, 1990; 1992). There is no evidence specifically regarding emotion and emotion regulation-related traits or behaviors predicting parental attributions of child behavior. However, existing research demonstrates that other negative child behaviors and traits 
related to the regulation of emotions or behavior that challenge parents are related to more negative and internal attributions (Miller, 1995). For example, parents have more internal than external attributions of adolescent depression (Chen, Johnston, Sheeber, \& Leve, 2009; Sheeber, Johnston, Chen, Leve, Hops, \& Davis, 2009), autism spectrum disorder (ASD; Whittingham, Sofronoff, Sheffield, \& Sanders, 2008), and attention deficit hyperactivity disorder (ADHD; Johnston \& Freeman, 1997).

Based on these established relations between psychopathology and internal attributions, the internal attributions of anger for negative emotionality reported here are likely a reflection of mothers' frustration, and potentially their own emotion dysregulation when dealing with their child's difficulty regulating their emotions. Given that anger has the potential to be very disruptive behaviorally (e.g., compared to shame, which would lead a child to avoid an emotionally stimulating situation), a child's natural tendency to be highly negative or emotional likely ups the ante in terms of how frustrated and upset parents can feel. Mothers' socialization goals with younger children are to maximize positive and neutral emotions, and minimize negative ones (Denham, 1993; Malatesta \& Haviland, 1982). If mothers have a similar socialization agenda for older children where their goal was to minimize their child's experience of negative emotions, if their child is constantly emotionally dysregulated and highly emotional (i.e., high in negative emotionality), mothers will experience frustration because their goal to reduce the child's negative emotion is being impeded. It makes sense that mothers would adopt more internal attributions of negative emotion displays in general given that parents hold more internal attributions of negative child traits or behaviors compared to positive ones (Gretarrson \& Gelfand, 1988; Miller, 1995). This may be particularly true for anger because it is disruptive and unpredictable (Izard \& Ackerman, 2000; Lewis, 2008; Miller, 1995). 
In the case of attention focusing and maternal attributions of anger, it may be that children's level of attention focusing is perceived by mothers as a negative trait, and this is the reason it was related to a lower level of maternal attributions of anger (i.e., indicative of internal attributions). Higher-order cognitive processes such as attention focusing or inhibitory control are helpful in early childhood for promoting the regulation of children's emotions and behavior (e.g., Carlson \& Wang, 2007; Kahle, Utendale, Widaman, \& Hastings, 2017). However, in some regulatory situations, these higher-order cognitive processes may actually hinder emotion regulation because they can over-orient the individual to cues of the distressing stimuli and lead to depletion of the individual's cognitive resources (Bardeen \& Stevens, 2015). If children are constantly over-attending to emotional stimuli to the point of consistent emotion dysregulation, this may be highly taxing for parents to attend to and address in daily socialization interactions. As noted above, if mothers' socialization goals are oriented around minimizing negative emotions (Denham, 1993; Malatesta \& Haviland, 1982), they are going to be very frustrated by their child's emotion dysregulation, and by their child's natural propensity of over-orienting toward emotional stimuli. As a result, it is likely mothers would adopt more internal attributions for these displays (Miller, 1995).

In addition, it is particularly interesting that all four of the temperamental traits were significantly related to maternal attributions for anger, and of all four emotions examined, anger was the only emotion where all four of the temperamental traits were significantly related to maternal attributions of dysregulated displays of that emotion. This is likely due to the fact that anger is behaviorally, physiologically, and psychologically activating for both child and parent, as well as the fact that children's anger is less predictable in terms of producing an approach or withdraw response from the child (Carver \& Harmon-Jones, 2009; Cooper et al., 2008). 
In addition, depending on several situational factors (e.g., the child's sensitivity to threat and reward), a child's experience of anger may lead them to either approach or withdraw from the emotional stimulus, rather than simple avoidance (Carver \& Harmon-Jones, 2009; Cooper et al., 2008). For example, high levels of behavioral activation system (BAS) scores are indicative of behaviors involved in approaching some desired incentive or goal (Carver \& Harmon-Jones, 2009). In addition, in young adult populations, the tendency to express anger is positively related to BAS scores (Smits \& Kuppens, 2005). If children are high in BAS scores, they are much more oriented toward potential rewards and reaching a goal. Therefore, they would be more likely to engage in approach behaviors when they experience anger (e.g., inflicting pain on the individual who is blocking the goal or offending them; Carver \& Harmon-Jones, 2009). This added level of complexity may make anger in particular more challenging for parents to address during daily socialization interactions; as a result, when addressing children's anger, parents may become more responsive to or affected by their child's nuanced temperamental traits that either promote or undermine emotion regulation processes. Because anger has the potential to be more unpredictable, in situations where parents are trying to make quick, in-the-moment socialization decisions in response to children's dysregulated anger, they may more heavily rely on children's varying temperamental traits. In these situations, children's temperamental traits communicate to parents areas where the child is vulnerable to dysregulation and provide information parents can use to guide their responses in the future (e.g., "my child has difficulty regulating X emotion in Y situation based on what happened with Z").

Fear and shame/embarrassment. For both fear and shame/embarrassment, the pattern of findings was identical; a high level of soothability and inhibitory control were related to a high level of maternal attributions of these emotions (i.e., indicative of external attributions). Of the 
four negative emotions examined in this study, fear and shame/embarrassment are the only two emotions that clearly lead to avoidance behavior and are physiologically activating. In addition, while sadness is clearly related to avoidance responses, it is not physiologically activating (Carver \& Harmon-Jones, 2009; Cooper et al., 2008; Welling, 2003). Taken together, these similarities may explain why the pattern of findings was identical for these two emotions and not for sadness and anger.

In addition, soothability and inhibitory control could be particularly helpful in regulating fear and shame/embarrassment. Soothability would help the child regulate the emotion more quickly, and inhibitory control would help the child mobilize their resources to address the distressing stimuli that is causing the emotion. If children are high on these traits, if they express fear or shame/embarrassment in a dysregulated way, it makes sense that mothers would draw more external attributions for these displays (e.g., the child did not mean to express the emotion in that way, the child never expresses the emotion in that way, the display was due to the situation). In addition, it may be these findings indicate that mothers are accurately assessing their child's regulatory patterns; if mothers perceive their child as being highly soothable in emotional situations and having the ability to stop themselves from engaging in behaviors they are not supposed to engage in (i.e., high in inhibitory control), if they do engage in a dysregulated display of fear or shame/embarrassment, mothers attribute it to more external causes because the behavior is atypical for their child (e.g., the dysregulated display was due to some sort of situational stressor; Root \& Rasmussen, 2017).

Patterns across specific emotions - predicting maternal attributions. The findings for attributions of fear and shame/embarrassment were identical; high levels of soothability and inhibitory control were related to a high level of maternal attributions of fear and 
shame/embarrassment (i.e., indicative of external attributions). Both emotions represent differing types of perceived threat (Lewis, 2008; Öhman, 2008); in the case of fear, the threat itself can be multifaceted and can come from several sources, but it represents some sort of real danger or threat that must be addressed (Öhman, 2008). In the case of shame/embarrassment, the threat being addressed is a particular part of the self or the entire self from a global perspective (Lewis, 2008). Furthermore, both emotions share similar practical function in that both clearly lead to withdrawal and avoidance behaviors (Izard \& Ackerman, 2000; Kelley et al., 2000). These findings are particularly interesting, given that these two emotions emerge from two different sources; in the case of shame/embarrassment, these emotions result from hostile or negative socialization behaviors that lead to negative evaluations of the self, while fear has a prominent dispositional basis (Izard \& Ackerman, 2000; Lewis, 2008; Rothbart \& Bates, 2006). It may be that although these two emotions originate from different sources, their similar response patterns of withdrawal and avoidance leads mothers to respond similarly in terms of their attributions of dysregulated displays of these emotions.

Taken together, this evidence is powerful because it demonstrates not only that children's emotion regulation in general is related to parental attributions about sadness, anger, fear, and shame/embarrassment, but it also demonstrates how multiple temperamental indices of emotion and emotion regulation-related traits impact parental attributions of specific negative emotions. These findings suggest that mothers themselves are not simply responding to children's emotion regulation in general, but to multiple different components of children's emotionality and regulatory patterns. 


\section{Research Question Two: Indirect Effects of Child Temperament on Maternal Emotion Socialization of Specific Emotions via Maternal Attributions of Specific Emotions}

The second research question examined whether dimensions of child temperament (i.e., negative emotionality, soothability, attention focusing, inhibitory control) were indirectly related to maternal emotion socialization responses to sadness, anger, fear, and shame/embarrassment via maternal attributions of each specific emotion. Previous research demonstrates that other behaviors influenced by temperamental traits (e.g., aggression, social withdrawal) are increasingly related to internal attributions as children get older (Mills \& Rubin, 1992); however, it was somewhat unclear how mothers would respond to these nuanced components of children's emotionality and emotion regulation behaviors, and how this might translate to maternal emotion socialization responses in response to specific emotions. It was hypothesized that the relations between the four dimensions of children's temperament and maternal supportive and nonsupportive emotion socialization responses to specific emotions would not be one-to-one, rather, it was expected that maternal attributions of specific emotions would indirectly affect this relation. In general, the pattern of findings was that one or more of the four temperamental traits indirectly affected nonsupportive responses to a particular emotion via maternal attributions of that emotion, but not for supportive responses. The presence of an indirect effect assumed the presence of a significant relation between the temperamental dimension and maternal attributions of the specific emotion ( $\mathrm{x}>\mathrm{m}$ path), and between maternal attributions of the specific emotion and maternal emotion socialization in response to the specific emotion ( $\mathrm{m}>\mathrm{y}$ path).

Maternal attributions of sadness. For sadness, all four of the temperamental traits (i.e., negative emotionality, soothability, attention focusing, inhibitory control) indirectly affected maternal nonsupportive responses to sadness via maternal attributions of sadness. Based on 
previous research documenting links between children's difficultness and internal attributions of behavior (e.g., Gretarrson \& Gelfand, 1988), it was hypothesized that all of the four temperamental dimensions would be related to maternal attributions in expected directions, and that negative emotionality would be negatively related with maternal attributions of sadness (i.e., indicative of internal attributions) and soothability, attention focusing, inhibitory control would be positively related to them (i.e., indicative of external attributions). For all four temperamental variables, maternal attributions of sadness were significantly and negatively related to nonsupportive responses to sadness; a high level of maternal attributions (i.e., indicative of external attributions) was related to a low level of nonsupportive responses to sadness. For maternal supportive emotion socialization responses to sadness, none of the temperament dimensions indirectly affected supportive responses to sadness. This and the other findings for supportive emotion socialization will be discussed below in the section of findings across emotions.

Maternal attributions of anger. Negative emotionality, attention focusing, and inhibitory control were indirectly related to nonsupportive responses to anger via maternal attributions of anger; high levels of negative emotionality were negatively related to maternal attributions of anger (i.e., indicative of internal attributions), and attention focusing and inhibitory control were positively related to maternal attributions (i.e., indicative of external attributions). For all three significant temperament variables, maternal attributions of anger were significantly and negatively related to nonsupportive responses to anger; a high level of maternal attributions of anger was related to a low level of nonsupportive responses to anger.

For supportive emotion socialization responses to anger, negative emotionality and attention focusing were indirectly related via maternal attributions of anger. Specifically, 
negative emotionality was negatively related to maternal attributions, and attention focusing was positively related to maternal attributions of anger. For the negative emotionality and attention focusing models, maternal attributions of anger were positively related to supportive emotion socialization responses to anger; a high level of maternal attributions of anger (i.e., indicative of external attributions) was related to a high level of supportive responses to anger. It is important to note here that the finding for attention focusing was opposite of research question one, which revealed a negative beta weight for attention focusing on the prediction of maternal attributions of dysregulated anger. Given that mothers and children did not complete identical batteries of surveys, and the surveys used in research question one were all maternal report, an augmented sample was used for research question one that utilized additional mothers who completed the surveys but their child did not. For research question two, the beta weight for attention focusing predicting maternal attributions of anger was positive; a high level of attention focusing was related to a high level of attributions of anger (i.e., indicative of external attributions). Post-hoc analyses revealed that the mothers in dyads $(n=123)$ reported significantly higher levels of child attention focusing $(M=.10, S D=.68)$ and maternal attributions of anger $(M=3.55, S D=.92)$ than mothers who completed the surveys on their own (attention focusing, $M=-.34, S D=.68$; attributions of anger, $M=3.03, S D=.80)$.

In addition, post-hoc analyses also explored whether additional demographic factors might have played a role in mothers' attributions of anger. Maternal race, education level, occupation status, and household income were tested as covariates. The only variable that approached significance was maternal education level $(p=.075)$. There were slightly more mothers in the group who completed the surveys without their child that had a university degree (50\% versus $43.9 \%$ for mothers who completed the surveys in a pair). However, for the group 
who completed the surveys in a pair with their child, there was a significantly higher proportion of mothers who held Master's degrees (22\%) and Doctoral degrees (11.4\%) compared with mothers who did not complete the surveys with their child (Master's degree, $8.3 \%$; Doctoral degree, $2.8 \%$ ).

Maternal attributions of fear. Attention focusing and inhibitory control were indirectly related to maternal nonsupportive responses to fear via maternal attributions of fear. Both attention focusing and inhibitory control were significantly and positively related to maternal attributions of fear; high levels of attention focusing and inhibitory control were related to a high level of maternal attributions (i.e., indicative of external attributions). In addition, for both models, maternal attributions of fear were significantly and negatively related to nonsupportive responses to fear. There were no indirect effects of child temperament for any of the supportive emotion socialization models for fear.

Maternal attributions of shame/embarrassment. The pattern of findings for emotion socialization responses to shame/embarrassment was identical to the pattern for sadness; all four temperament dimensions were indirectly related to nonsupportive responses to shame/embarrassment via attributions of shame/embarrassment. Negative emotionality was negatively related to maternal attributions of shame/embarrassment; a high level of negative emotionality was also related to a low level of maternal attributions of shame/embarrassment (i.e., indicative of internal maternal attributions). Interestingly, for the negative emotionality model, higher levels of maternal attributions (i.e., indicative of external attributions) were related to higher levels of nonsupportive responses to shame/embarrassment. Across all the analyses, this was the only finding that linked external attributions with nonsupportive emotion socialization responses. Compared to other basic emotions of sadness, anger, and fear, 
shame/embarrassment is a self-conscious emotion that requires more complex cognitive processes (Lewis, 2008). In addition, because negative evaluations of the self, rather than other people or other external factors, are involved with shame/embarrassment, the experience of this emotion is highly physiologically and emotionally arousing, and can lead to a disruption in both thought and behavior different than the experience of sadness, anger, and fear (Lewis, 2008). As a result, even if a mother strongly attributed a child's dysregulated expression of shame/embarrassment as being the result of external attributions (e.g., the child did not mean to express the emotion in that way, the expression was due to the situation, etc.), it may be that shame/embarrassment is so intense or frustrating to deal with that it leads a mother to respond in more nonsupportive ways, even when she understood there were external reasons for the child responding that way.

For soothability, attention focusing, and inhibitory control, all three variables were significantly and positively related to maternal attributions of shame/embarrassment; high levels of all three traits were related to a high level of maternal attributions (i.e., indicative of external attributions). In turn, maternal attributions of shame/embarrassment for these models (i.e., soothability, attention focusing, and inhibitory control) were negatively related to nonsupportive responses to shame/embarrassment; a high level of maternal attributions (i.e., indicative of external attributions) was related to a low level of nonsupportive responses to shame/embarrassment. In contrast to the model for negative emotionality, for the soothability, attention focusing, and inhibitory control models, high levels of maternal attributions for anger (i.e., indicative of external attributions) were related to lower levels of nonsupportive emotion socialization responses to anger. If mothers attribute children's displays of shame/embarrassment to external factors (e.g., the child did not mean to express the emotion in that way, the expression 
of the emotion was due to the situation), they may see less reason to respond in nonsupportive ways or use nonsupportive responses as a strategy less often. It may be in these situations, mothers see these external factors as extenuating circumstances that caused the child to express shame/embarrassment in a dysregulated way, and as a result, they do not feel nonsupportive responses are needed. In this way, external attributions of shame/embarrassment may serve as a protective factor against nonsupportive responses that could significantly damage a child's emotion regulation abilities (Buckholdt et al., 2014; Hurrell et al., 2015; Sheffield Morris et al., 2007).

Patterns across emotions - indirect effects of child temperament Across all four emotions, any of the significant temperamental traits were related to maternal attributions in the following pattern: negative emotionality was negatively related to maternal attributions (i.e., indicative of internal attributions; for sadness, anger, and shame/embarrassment), and any of the remaining three temperamental traits (i.e., soothability, attention focusing, and inhibitory control) were positively related to maternal attributions (i.e., indicative of external attributions). As discussed with research question one, these findings primarily support explanations of parental attributions that posit that parents hold more internal attributions of more negative child behaviors or traits, and in turn, external attributions of more positive ones (Gretarrson \& Gelfand, 1988; Miller, 1995). Soothability, attention focusing, and inhibitory control all promote the regulation of negative emotions (Rothbart \& Bates, 2006). The items in the maternal attributions measure asked mothers about their child's dysregulated display of the particular emotion. If mothers perceived their child as being high on these traits that promote their emotion regulation, it makes sense they would draw external attributions (e.g., the expression was due to 
the situation, my child never expresses the emotion in that way) if they saw that child express a particular negative emotion in a dysregulated way.

In terms of the relations between the maternal attributions of the specific emotions and nonsupportive emotion socialization, all significant relations between maternal attributions and nonsupportive emotion socialization responses were negative with one single exception is discussed below; a high level of attributions (i.e., indicative of external attributions) was related to a low level of nonsupportive emotion socialization. A high level of external attributions was indicative of the mother attributing the dysregulated negative emotion to a phase, the child did not intend to express the emotion in that way, they never express the emotion in that way, and the way they expressed the emotion was due to the situation. Because mothers attributed these dysregulated emotion displays to causes external to their children, they may not have been as frustrated by them, and used less nonsupportive emotion socialization responses as a result.

Interestingly, the single exception for this pattern was the link between maternal attributions and nonsupportive emotion socialization responses to shame/embarrassment for the negative emotionality model; in this case, a high level of maternal attributions (i.e., indicative of external attributions) was positively related to nonsupportive emotion socialization. In the case of supportive emotion socialization, maternal attributions of anger were positively related to supportive emotion socialization responses to anger; a high level of maternal attributions (i.e., indicative of external attributions) was positively related to supportive emotion socialization responses to anger.

In addition, it is interesting to note the findings for maternal attributions of sadness and shame/embarrassment were identical to one another; for both emotions, attributions of each emotion were indirectly affected the relation between all four temperamental traits and 
nonsupportive emotion socialization responses to the emotion. While there are differences in the physiological profiles for both emotions, from a behavioral perspective, both emotions are markedly less behaviorally activating than anger and lead to withdrawal and avoidance behaviors (Kelley et al., 2000; Smiley et al., 2016). Perhaps because both emotions functionally lead children to behaviorally shut down and avoid the source of the emotional stimuli distressing them, this may create a potentially unique and challenging parenting scenario. When mothers are responding to children's sadness or shame/embarrassment, mothers are faced with the opposite problem they might have when responding to children's anger. With anger, although it may be less likely with older children, there is the potential parents may be forced to restrain their child or stop them from engaging in a dysregulated behavior; whereas with sadness and shame/embarrassment, parents may have the opposite problem of trying to get the child to address the stimulus that is distressing them (e.g., doing something that is making them feel sad or ashamed/embarrassed). In these types of situations where the child's behavior may be grounded in inaction, as noted with the findings for anger for research question one, parents may be even more naturally responsive to the nuances in their child's temperamental profile. Specifically, they may rely more on their child's temperamental traits for information to inform their attributions of their child's displays of these emotions, and, in turn, their nonsupportive responses to these dysregulated displays.

Supportive versus nonsupportive emotion socialization responses. The overwhelming pattern of findings for research question two was that maternal attributions of the specific emotions were indirectly related to the relations between the four temperamental traits (i.e., negative emotionality, soothability, attention focusing, and inhibitory control) and nonsupportive emotion socialization responses to the specific emotions, but not for supportive responses. The 
only exception for this was for maternal attributions of anger; for the negative emotionality and attention focusing models, maternal attributions of anger were indirect effects. The fact that the pattern of findings was so clearly differentiated between nonsupportive and supportive suggests that the two types of responses are distinct subscales rather than two opposite ends of a continuum (Fabes et al., 2002). In addition, Nelson, O’Brien, Blankson, Calkins, and Keane (2009) found that different parental stress factors (e.g., partner job satisfaction) affect parental supportive and nonsupportive responses differently. Furthermore, when nonsupportive emotion socialization responses are being measured, it is likely that these responses are potentially tapping into parental frustration or negative feelings about their child's emotion regulation behaviors or patterns. If this is the case, it may be that for nonsupportive versus supportive emotion socialization, mothers are particularly influenced by their child's temperamental traits that make emotion socialization easier or more difficult, and this will vary for nonsupportive and supportive responses. In these situations, children's temperamental traits may be even more influential, affecting maternal attributions and resulting emotion socialization responses.

In addition, as noted above, the only exception to the overwhelming significant findings for nonsupportive responses was for maternal attributions of anger; maternal attributions of anger indirectly affected the relations between child negative emotionality and attention focusing and supportive responses to anger. This lack of significant findings for supportive emotion socialization may represent the resilience of these types of responses. It may be that when parents experience the stresses of addressing their children's negative emotions, they continue to engage in supportive behaviors that they were already using previously and were effective for helping them respond to their child (Rasmussen, Hartman, \& Root, 2017). It may also be that mothers who engage in supportive emotion socialization responses are more resilient or have 
more resources with which to respond to their child's temperament and emotional experiences (Rasmussen et al., 2017).

\section{Research Question Three: Maternal Emotion Socialization as Moderator Between Child Temperament and Emotion Outcomes}

The third research question examined the relation between child temperament, maternal supportive and nonsupportive emotion socialization responses to sadness, anger, fear, and shame/embarrassment, and three child emotion outcomes: cognitive reappraisal, and expressive suppression, and regulatory emotional self-efficacy. While main effects were expected (i.e., temperament dimension $>$ emotion outcome; maternal emotion socialization $>$ emotion outcome), it was hypothesized these effects would be subsumed when interaction terms (temperamentXemotion socialization response to specific emotion) were included in the individual regression models. The rationale behind this was that supportive emotion socialization responses promote healthier emotion outcomes (i.e., cognitive reappraisal, regulatory emotional self-efficacy) and buffer against the use of expressive suppression (Buckholdt et al., 2014; Hurrell et al., 2015; Sheffield Morris et al., 2007); however, the overwhelming pattern was maternal supportive and nonsupportive emotion socialization responses to specific emotions did not moderate the relations between child temperament and the emotion outcomes. Findings are discussed in detail below.

Cognitive reappraisal. For the model examining cognitive reappraisal in relation to emotion socialization of sadness, inhibitory control was the only significant predictor; a high level of inhibitory control was related to a high level of cognitive reappraisal. For the cognitive reappraisal in relation to emotion socialization of anger and fear models, child inhibitory control and maternal supportive responses to anger were significantly and positively related to cognitive 
reappraisal; high levels of inhibitory control and maternal supportive responses to anger were related to high levels of cognitive reappraisal. For the model for cognitive reappraisal in relation to emotion socialization of shame/embarrassment, the findings mirrored that of emotion socialization of sadness; a high level of inhibitory control was related to a high level of cognitive reappraisal.

These findings for inhibitory control for the sadness, anger, fear, and shame/embarrassment models make sense within the context of inhibitory control's function to help a child inhibit a dominant response in service of a more adaptive, subdominant one (Rothbart \& Bates, 2006). If a child is high inhibitory control and can effectively control their behavior, they are likely to engage in more socially and emotionally adaptive behavior (Calkins \& Dollar, 2014; Kim \& Cicchetti, 2010). This leads a child to feel more regulated emotionally, and when a child is more emotionally regulated, this frees up cognitive resources with which to reappraise emotionally evocative situations (e.g., "because I'm able to manage my emotions, I have more cognitive space to address X emotional stimuli"; Calkins \& Dollar, 2014). These relations are further discussed below in the discussion of patterns across emotions.

Patterns across emotions - cognitive reappraisal. The cognitive reappraisal and emotion socialization responses to anger and fear were identical; for both emotions, high levels of inhibitory control and supportive responses to each emotion were related to a higher level of cognitive reappraisal. Both emotions are behaviorally and physiologically activating for children (Lemerise \& Dodge, 2008; Öhman, 2008), and children themselves report anger is harder to control compared to sadness (Zeman \& Shipman, 1997). In regulatory situations, children expect less support from parents in regulating anger (Zeman \& Shipman, 1996), and report hiding anger more than other emotions (Underwood, 1997). As a result, anger may be particularly disorienting 
to children's emotion regulation patterns. This could be the case not only because anger is behaviorally activating (Lemerise \& Dodge, 2008), but also unpredictable (Smiley et al., 2016). These effects could be amplified if children do not expect support or assistant in managing their anger (e.g., "I'm feeling angry but I don't think I'll have help in dealing with it").

In addition to inhibitory control, which was significant for all four emotions, in situations where children are experiencing anger or fear as opposed to sadness or shame/embarrassment, maternal supportive emotion socialization responses were statistically significant. For anger and fear only, maternal supportive responses were related to higher levels of child cognitive reappraisal. This is supported by existing literature in this area that demonstrates supportive emotion socialization responses are related to children's effective emotion regulation (Buckholdt et al., 2014; Hurrell et al., 2015). Supportive emotion socialization responses not only decrease children's emotional and physiological arousal and help comfort the child, but provide opportunities for parents to teach the child about specific emotion regulation strategies to manage negative emotions (Sheffield Morris et al., 2007). When parents engage in supportive emotion socialization behaviors, children are better emotionally regulated (Buckholdt et al., 2014; Hurrell et al., 2015). When children are more physiologically and emotionally regulated, they have more cognitive resources at their disposal to initiate and use more adaptive emotion regulation strategies such as cognitive reappraisal (Calkins \& Dollar, 2014). This happens because cognitive resources that would otherwise be used toward regulating an emotion can then be put toward engaging in higher-order cognitive processes that further promote children's emotion regulation efforts (Calkins \& Dollar, 2014).

In addition, the findings for cognitive reappraisal and emotion socialization responses to sadness and shame/embarrassment were also identical to one another. For both emotions, child 
inhibitory control was the sole temperamental predictor of child cognitive reappraisal. Both emotions are clearly related to an avoidance orientation toward distressing stimuli (Kelley et al., 2000; Smiley et al., 2016). In addition, unlike anger and fear, fear and shame/embarrassment are behaviorally deactivating and can cause a child to want to hide or stop engaging in certain cognitive and motor behaviors (Izard \& Ackerman, 2000; Lewis, 2008). Because these two emotions are related to a sense of inactivity (e.g., withdrawal orientation, lack of behavioral response), it could be that inhibitory control is uniquely beneficial in facilitating certain behaviors, rather than inhibiting them. For example, traditionally, we think of inhibitory control as preventing maladaptive behaviors such as aggression (e.g., Utendale \& Hastings, 2010) and impulsivity (Olson, Schilling, \& Bates, 1998). In the case of sadness and shame/embarrassment, inhibitory control could lead children to engage in more adaptive behaviors in response to these emotions, rather than simply avoiding them. Whereas with other behaviors, inhibitory control means stopping a negative behavior, in this sense, inhibitory control helps curb the dominant response of inaction or avoiding and helps the child to take action and engage in behaviors that promote emotion regulation such as cognitive reappraisal.

It is interesting to note across all four emotions, only two variables were significantly related to cognitive reappraisal in relation to each emotion: child inhibitory control and supportive responses to anger and fear. As discussed above, this clearly suggests inhibitory control plays an integral role in children's cognitive reappraisal, but it is interesting negative emotionality, soothability, and attention focusing or supportive responses to any of the emotions did not predict it. This suggests that there may be other aspects of children's temperament that may promote the use of cognitive reappraisal (e.g., impulsivity), or alternatively, there may be other variables external to the child that may predict cognitive reappraisal use. For example, 
Gunzenhauser and colleagues (2014) found that in addition to supportive emotion socialization responses, parental modeling of cognitive reappraisal was significantly related to its use by children. Taken together, more research is needed to understand factors that contribute to the use of cognitive reappraisal in older children.

Expressive suppression. For expressive suppression in relation to emotion socialization of all four emotions, maternal supportive responses to the emotion were significantly related to low levels of expressive suppression. It is likely that when mothers are using a high level of supportive responses, there is less of a need for children to engage in expressive suppression in the first place because the supportive emotion socialization has either decreased the child's level of emotional arousal or actually taught or communicated to the child how regulate it (Buckholdt et al., 2014; Hurrell et al., 2015). In the emotion socialization measure used for this study, supportive emotion responses included behaviors that both were aimed at reducing the child's distress (e.g., physically comforting the child) and helping the child generate constructive ideas about how to manage their emotions. When children receive these types of responses from mothers, this buffers against the need to use expressive suppression to manage powerful negative emotions.

Patterns across emotions - expressive suppression. In addition, there were significant effects for child sex. For expressive suppression in relation to emotion socialization responses to sadness, anger, and shame/embarrassment, female sex was significantly related to a low level of expressive suppression. This is in line with existing research documenting that boys utilize expressive suppression more often than girls (Gross \& John, 2003). This may be because of gendered socialization practices that encourage males to suppress emotional experiences rather 
than engage with and reflect upon them as is done with girls (Bardeen \& Stevens, 2015; NolenHoeksema, 2012).

It is particularly interesting that the only significant temperament variable that predicted expressive suppression was inhibitory control for expressive suppression in relation to emotion socialization responses to shame/embarrassment. Surprisingly, a high level of inhibitory control was related to a high level of expressive suppression. Inhibitory control has clear purpose in helping promote the regulation of both behavior and emotion processes in children (Hudson \& Jacques, 2014; Schachar \& Logan, 1990). However, it may be that in the case of emotions and regulatory processes, a high level of inhibitory control may reflect a level of over-control. In these situations, inhibitory control may inhibit the full experience and expression of negative emotions. This may lead a child to engage in behaviors that shut down these emotions. In addition, in other forms of psychopathology such as perfectionism and eating disorders, altered behavioral control in the form of overcontrol is implicated (Bartholdy, Campbell, Schmidt, \& O’Daly, 2016; Flett, Hewitt, Oliver, \& Macdonald, 2002). In the case of anxiety, a construct related to emotion regulation, high levels of inhibitory control and abstract thinking are related to poorer emotion clarity and higher levels of anxiety symptoms, particularly in women (Bardeen \& Stevens, 2015). Conceptually, this is somewhat counterintuitive because we would assume inhibitory control would help buffer against the development of anxiety or emotion dysregulation. However, as noted above in research question one, in these situations, high levels of inhibitory control or other higher-order cognitive processes (e.g., attention focusing) can actually inhibit emotion regulation and lead to higher emotional arousal and dysregulation (Bardeen \& Stevens, 2015). If the child is high in inhibitory control and constantly attending to and reflecting upon distressing emotional cues, this very likely leads the child to be 
overwhelmed and emotionally dysregulated and use suppression in an attempt to calm themselves by shutting the experience down, particularly so in the case of shame/embarrassment because it is exceptionally emotionally distressing compared to sadness, anger, and fear (Lewis, 2008).

Regulatory emotional self-efficacy. Findings within and across emotions are discussed in detail below for regulatory emotional self-efficacy.

Sadness and fear. There were no significant predictors of regulatory emotional selfefficacy of negative emotions for the sadness and fear models; the model for sadness was not significant, and for fear, the overall regression model was significant, but none of the predictors were. This was surprising, given that it could be assumed that temperamental traits related to children's emotionality and regulatory patterns would be related to children's sense of selfefficacy about their regulatory efforts. It may be that the relation between children's emotionality and regulatory patterns and their sense of regulatory self-efficacy is not simply one-to-one; there could be additional variables that may explain the disconnect between children's emotionality and regulatory patterns and their regulatory emotional self-efficacy. For example, parents' thoughts and cognitions are subject to several biases (e.g., feeling negative emotions; Dix, Reinhold, \& Zambarano, 1990) that can lead parents to communicate inaccurate or maladaptive messages about their child to their child. As a result, children themselves may internalize these potentially harsh or inaccurate messages given to them from their parents about their emotionality and regulatory patterns, making temperamental measurements of these constructs non-significant. In addition, children's self-esteem could influence the ways they think about themselves and their regulatory patterns and competencies (Nader-Grosbois, 2014; Kolubinski, Nikcevic, Lawrence, \& Spada, 2016). 
Anger. For emotion socialization responses to anger, supportive responses to anger were the only variable significantly related to regulatory emotional self-efficacy. Given that anger can be a physiologically and behaviorally disruptive emotion for both parents and children, the findings of this study suggest supportive responses to this emotion are particularly crucial over nonsupportive ones (Heubner \& Izard, 1998; Izard \& Ackerman, 2000; Lemerise \& Dodge, 2008). Supportive emotion socialization responses help children learn actual skills and strategies to regulate their emotions (Sheffield Morris et al., 2007). Having these skills and strategies in one's emotional repertoire can be very competence-affirming for a child (e.g., "I know how to use X skill or strategy, and it helps me calm down"). Maternal supportive responses also decrease a child's actual level of emotion dysregulation (Buckholdt et al., 2014; Hurrell et al., 2015). If a child feels more regulated, they are also more likely to accurately ascertain their sense of regulatory self-efficacy.

Shame/embarrassment. For regulatory emotional self-efficacy in relation to emotion socialization responses to shame/embarrassment, nonsupportive responses were related to a lower level of regulatory emotional self-efficacy. When mothers engage in nonsupportive emotion socialization responses (e.g., punishing the child for expressing shame/embarrassment), these behaviors likely communicate to children mothers' perception of the child's ability to regulate (e.g., "if my mom says I should be ashamed for feeling this way, then I'm probably not good at managing my feelings"). In addition, when mothers respond to children's displays of shame/embarrassment in nonsupportive ways, they are not providing children with effective modeling and teaching of regulatory skills and strategies. As a result, children may internalize potentially inaccurate and negative messages about their regulatory abilities communicated from mothers via nonsupportive emotion socialization responses, thereby decreasing their regulatory 
emotional self-efficacy (Sheffield Morris et al., 2007). In addition, while the mothers in this investigation were overall quite supportive, high levels of maternal nonsupportive responses may be emotionally dysregulating for children, and as a result, it may be very difficult for them to feel like they are doing well are regulating or are in a position to practice effective emotion regulation strategies.

The single significant interaction term for all the models in research question three was the interaction between negative emotionality and nonsupportive responses to shame/embarrassment. This interaction was probed, and at medium and high levels of nonsupportive responses to shame/embarrassment, negative emotionality was significantly related to a low level of regulatory emotional self-efficacy. In addition, children who were lowest in negative emotionality and reported their mothers engaged in the highest levels of nonsupportive emotion socialization responses to shame also reported the highest levels of regulatory emotional self-efficacy. Given the high volume of potential interaction terms tested for research question three, it is likely this significant single interaction was a type one error, meaning that the significant interaction term was a false positive and the null hypothesis was incorrectly rejected (Field, 2013).

\section{Limitations and Future Directions}

This study makes an important contribution to the literature in this area in several ways, including the examination of both parent and child antecedents of maternal emotion socialization and children's emotion outcomes and the use of both parent and child report. In addition, the use of a specific emotions approach and multiple indices of children's emotionality and emotion regulation patterns are also important. However, much more research is needed to understand 
both child and parent contributions to maternal attributions, emotion socialization responses, and children's emotion-related outcomes.

Complexity of socialization agents in children's experiences. For the purposes of this study, socialization was examined from the perspective of children and parents as critical contributing socialization agents (Kuczynski \& Parkin, 2007). While both socialization agents clearly play a critical role in facilitating children's outcomes, children's trajectories are also influenced by additional socialization agents, such as peers and teachers. Once children enter school, the number of hours spent with peers drastically increases, and hours spent with parents decreases (Bukowski, Brendgen, \& Vitaro, 2015). When children are in shared space with peers at school, values and expectations for appropriate behavior are communicated between children, and peers provide assistance, advice, and coaching to one another (Wentzel \& Looney, 2015). In terms of emotions specifically, as children age, they prefer to disclose emotional information to peers rather than adults and become more protective of friends' emotions versus parents, suggesting that peer relationships play a clear role in socializing older children's emotions (Saarni, 1988; Zeman \& Shipman, 1997).

In addition to peers, teachers and the school setting itself represent important socialization agents in children's trajectories. Teachers are not only responsible for communicating knowledge in important subject areas, but also to communicate and reinforce appropriate behavioral and social outcomes (Wentzel \& Looney, 2015). In addition, children's interpersonal relationships with teachers have the potential to provide a critical context for children to experience emotional security and practice emotional and social skills that will benefit them emotionally, socially, and academically (Pianta, Hamre, \& Stuhlman, 2003; Wentzel \& Looney, 2015). In the future, further research is needed to explore how these 
important socialization agents beyond parents and children work together or against one another to shape children's emotion outcomes.

Emotions as a Child Inventory. The exploratory factor analyses run for the items in the Emotions as a Child Inventory importantly demonstrated there were differences in the factor structure for each of the four emotions examined (i.e., sadness, anger, fear, shame/embarrassment). There were clear similarities in the sub-types of supportive and nonsupportive emotion socialization responses and these were used to create the final supportive and nonsupportive scales used in the analyses for research questions two and three. It is important to note that the specific items for the supportive and nonsupportive subscales varied slightly across emotions. For example, for the nonsupportive scale for sadness, three of the five items involved maternal ignoring behaviors, and the remaining two items involved punitive behaviors directed towards the child (e.g., giving the child a disgusted look). For anger, only one of the items involved ignoring behavior and the remaining three items were more punitive behaviors (e.g., telling the child they should be ashamed). These findings are important because they demonstrate that children's perceptions of what constitutes supportive and nonsupportive emotion socialization responses vary depending on the emotion that is being socialized. Future research is needed to further explore if these differential factor structures are present in other samples of children, and if similar patterns exist with specific positive emotions as well.

Cultural and socioeconomic diversity. It is important to acknowledge the sample for this study was primarily Caucasian, educated, and middle-to-upper class mothers and children. Parental emotion socialization responses take place within a complex system of factors and influences, and culture and social class are important distal factors that shape the context of parental socialization (Cole \& Tan, 2015; Wilkins \& Pace, 2014). At conceptual and practical 
levels, culture shapes parental beliefs about emotions themselves, how they are expressed, and the ideal ways they are regulated (Cole \& Tan, 2015; Friedlmeier, Corapci, \& Cole, 2011). For example, in collectivist cultures oriented toward group rather than individual harmony, children's negative emotions are treated as extremely undesirable and damaging to interpersonal harmony, and as a result, must be strictly controlled (Chen, 2000; Raval \& Martini, 2011; Raval, Daga, Raval, \& Panchal, 2016; Wang, 2013). Further research is needed to explore if or how these differing beliefs translate to parental emotion socialization responses, and how those responses shape children's emotional adjustment within and across cultures.

In addition, social class also influences the context of parental socialization. Parents who experience financial hardship may have different socialization beliefs, goals, and resources generally and related to emotions specifically (Wilkins \& Pace, 2014). In addition, the stigmatization of these difficulties and resulting emotional distress influence the context of parental socialization (Wilkins \& Pace, 2014). As a result, in the future, it is critical to further understand how emotion socialization responses function and are related to adjustment in populations that are currently understudied, such as families of color, or those who are socioeconomically vulnerable.

Use of mother and child report, shared method variance. The use of both mothers and children as informants to examine emotion socialization responses was a unique strength of this study; however, both reporters are subject to bias, and as a result, there may have been mismatch between mothers and children's perceptions of the traits and behaviors of the other. In the future, it is important to have both mothers and children complete surveys assessing maternal emotion socialization, so that their responses can be directly compared with one another. In addition, both mothers and children completed questionnaires for this study, but both reporters did not complete 
identical survey instruments. As a result, in the analyses for research question one, only mothers completed the surveys assessing child temperament and maternal attributions of children's negative emotion displays. In addition, the analyses conducted for research question one may have been subject to shared method variance. In the future, methods other than questionnaire instruments would be particularly helpful in allowing both mothers and children to speak about emotion socialization processes, such as a parent-child discussion task.

Cross-sectional nature of study. This study was not longitudinal in nature, and as a result, true transactional conclusions cannot be drawn. The time period examined in this study is particularly important because it is critical for better or for worse in preparing children for the transition to adolescence and middle or junior high school. For example, the transition to middle and junior high school presents challenges in multiple domains of children's functioning, and emotional difficulties during this period can last through adulthood (Eccles et al., 1993; Kansky et al., 2016; Roeser \& Eccles, 1998). However, in the future, it is important to examine how these variables operate longitudinally through the transition to adolescence, and if there are factors that may promote resilience and buffer against the negative effects of the adolescent transition.

Fathers. Importantly, fathers also make a distinct contribution to children's emotion experiences, and it is critical to understand how the relations between children's temperament, parental attributions, and children's emotion outcomes operate with fathers compared to mothers (Brand \& Klimes-Dougan, 2010; Denham \& Kochanoff, 2002; Garside \& Klimes-Dougan, 2002; Klimes-Dougan et al., 2007). In particular, given that fathers serve a unique role in exposing children to new experiences and stimuli, they are important agents in helping children regulate the emotional responses associated with these exposures (Paquette, 2004; Parke, 1996). In terms 
of emotion socialization specifically, fathers also may play an important role in helping socialize the development of emotion regulation, while mothers are more oriented toward helping children understand the nature of emotions and emotion cognition (Denham \& Kochanoff, 2002). Furthermore, the socialization role of fathers is particularly important during the period of adolescence as fathers play an important role in creating a coordinated (or not) parenting effort alongside mothers in facilitating adolescent outcomes (Brand \& Klimes-Dougan, 2010)

Inclusion of positive emotions. For the purposes of this study, only specific negative emotions were examined. Negative emotions are critically important to the socialization and development of emotion regulation. However, positive emotions represent an equally important component of children's emotional experiences that relate to both positive and negative child outcomes (Fredrickson, 1998; Katz et al., 2011; Yap, Allen, \& Ladouceur, 2008; Yi, Gentzler, Ramsey, \& Root, 2016). While the focus in the developmental literature is most often on negative emotions (Sheffield Morris et al., 2007), positive emotions can not only help improve children's well-being and provide important tools with which to regulate negative emotions, but a lack of parental encouragement of children's positive affect can lead to poorer child outcomes (Fredrickson, 1998; Yi et al., 2016). There has been a surge in research regarding the nature and effect of positive emotions on children's outcomes; however, further understanding regarding how these relations function in samples of older children and adolescents is critical (Katz et al., 2014; Yi et al., 2016). In addition, it is important to further understand whether maternal attributions of specific positive emotion displays are affected by children's temperament in similar or different ways compared to negative emotions.

Adaptation of Regulatory Emotional Self-Efficacy (RESE) scale. The measure that was used to assess children's regulatory self-efficacy beliefs was originally written in Italian, and 
has only been previously used with adolescents (Bandura et al., 2003) and with emerging adults

(Caprara et al., 2008). The wording of the questions was slightly changed to be more

developmentally appropriate for use with older children (e.g., "How well can you express happiness when something good happens to you?" versus "How good are you at expressing happiness when something good happens to you?"; Rasmussen, 2015). While the adaptation was not markedly different than the original measure, the fact that alpha reliability coefficients are unavailable in English to begin with, and the adaptation was used without that reliability information, suggests that more evidence is needed in the future to ascertain if the questions on the original measure are reliable, as well as the adaptation for use with older children.

\section{Applied Implications and Conclusions}

There are clear practical implications of this study that have the potential to positively impact parent-child socialization interactions in daily life. The findings linking dimensions of children's temperament and maternal attributions of specific emotions (i.e., research question one) and findings identifying indirect effects of child temperament on maternal emotion socialization via maternal attributions of specific emotions (i.e., research question two) provide important information that can shape both parenting interventions and actual parent-child socialization interactions.

For example, knowing how maternal attributions are affected by different temperamental dimensions and that more external attributions of negative emotion displays protect against the use of nonsupportive responses to specific negative emotions provides important information about attributions that parents can be taught about and practice in socialization interactions with children (Whitman et al., 1987). Teaching parents to pause and assess if a particular attribution about a behavior is fully adaptive or fitting for situation at hand can help parents assess if there 
are external attributions that might account for the child's behavior (e.g., if the child was tired, sick or otherwise provoked) rather than just resorting to thinking "my child is X way and I cannot do anything about it" and responding negatively. In addition to teaching parents to reflect upon their attributions and consider alternative sources for a child's behavior, children can also play a role in shaping and promoting positive parent-child interactions. Teaching children to reflect upon their temperamental characteristics and regulatory patterns can help them identify areas where they are more vulnerable to regulatory difficulties or have potential to improve.

In these ways, both parents and children can serve as important sources of resilience in parent-child interactions (Walsh 2012). Rather than viewing parent-child interactions from a deficit-based approach, a resilience-based perspective emphasizes the potential for both children and parents to rally their strengths together to promote positive outcomes for both children and parents. In addition, if both children and parents are taught that children's emotion competence can be taught and developed (i.e., a growth mindset; Dweck, 2006), this may naturally protect against internal attributions that reflect a fixed mindset (e.g., "This is just the way my child is, I cannot do anything about it"), and lead parents to adopt more external attributions for their child's developing emotional competence or regulatory difficulties.

Emotion socialization processes a critical task of parenting throughout childhood, and parents' behaviors powerfully shape children's emotional outcomes (Denham et al., 2015; Sheffield Morris et al., 2007). Both children and parents are active contributors to these processes, and as such, it is critical to examine how both shape the parent-child relationship (Kuczynski \& Parkin, 2007). Findings of this study suggest that multiple dimensions of children's temperament (i.e., negative emotionality, soothability, attention focusing, and inhibitory control) are related to maternal attributions of specific emotions, and these attributions 
indirectly effect the relation between children's temperament and maternal nonsupportive (and to a lesser degree, supportive) emotion socialization responses to specific emotions. Understanding these relations is important because it helps identify maternal attributions of specific emotions that place a child at-risk for receiving nonsupportive emotion socialization responses. Once identified, attribution retraining can promote more optimal parental socialization responses (Whitman, Fanshel, \& Grundy, 1987) Finally, findings of this study also suggested that supportive responses to some specific emotions help promote cognitive reappraisal and regulatory emotional self-efficacy and buffer against the use of expressive suppression.

This study added to the literature in several ways, particularly through the examination of both child and parent antecedents of parental attributions and emotion socialization responses and the use of both parent and child report. In addition, given that each negative emotion has a distinct purpose and profile (Izard \& Ackerman, 2000), the use of a specific emotions approach rather than negative emotion aggregate was beneficial. Examining maternal attributions and emotion socialization from a specific emotions context provided important information about ways mothers' attributions of children's displays of each emotion were different from and similar to one another. Furthermore, the examination of multiple aspects of children's emotionality and emotion regulation rather than a single index of emotion regulation were also particularly important. Examination of emotion regulation in this way allowed for a more differentiated perspective regarding how multiple aspects of children's emotion regulation rather than emotionality only shape parental attributions of children's specific emotion displays. Future research in this area should continue to explore the important ways parents and children dynamically contribute to ongoing parent-child interactions. 


\section{References}

Bandura, A. (2001). Social cognitive theory: An agentic perspective. Annual Review of Psychology, 52, 1-26. doi: 10.1146/annurev.psych.52.1.1

Bandura A. (1997). Self-efficacy: The exercise of control. New York: Freeman.

Bandura, A., Caprara, G. V., Barbaranelli, C., Gerbino, M., \& Pastorelli, C. (2003). Role of affective self-regulatory efficacy in diverse spheres of psychosocial functioning. Child Development, 74(3), 769-782. doi: 10.1111/1467-8624.00567

Barber, B. K., Stolz, H. E., \& Olsen, J. A. (2005). Parental support, psychological control, and behavioral control: Assessing relevance across time, method, and culture. Monographs of the Society for Research in Child Development, 70(4), 58-72.

Bardeen, J. R., \& Stevens, E. N. (2015). Sex differences in the indirect effects of cognitive processes on anxiety through emotion regulation difficulties. Personality and Individual Differences, 81, 180-187. doi: 10.1016/j.paid.2014.07.009

Barnett, V., \& Lewis, T. (1978). Outliers in statistical data. New York: Wiley.

Baron, R. M., \& Kenny, D. A. (1986). The moderator-mediator variable distinction in social psychological research: Conceptual, strategic, and statistical considerations. Journal of Personality and Social Psychology, 51(6), 1173-1182. doi: 10.1037/0022-3514.51.6.1173

Bartholdy, S., Campbell, I. C., Schmidt, U., \& O’Daly, O. G. (2016). Proactive inhibition: An element of inhibitory control in eating disorders. Neuroscience and Biobehavioral Reviews, 71, 1-6. doi: 10.1016/j.neubiorev.2016.08.022

Bates, J. E., Schermerhorn, A. C., \& Petersen, I. T. (2012). Temperament and parenting in developmental perspective. In M. Zentner \& R. L. Shiner (Eds.), Handbook of temperament (pp. 425-441). New York: Guilford Press. 
Bell, K. L., \& Calkins, S. D. (2000). Relationships as inputs and outputs of emotion regulation. Psychological Inquiry, 11(3), 160-209. doi: 10.1207/S15327965PLI1103_04

Bell, R. Q., \& Chapman, M. (1986). Child effects in studies using experimental or brief longitudinal approaches to socialization. Developmental Psychology, 22(5), 595-603. doi; $10.1037 / 0012-1649.22 .5 .595$

Belsky, J. (1984). The determinants of parenting: A process model. Child Development, 55(1), 83-96. doi: $10.2307 / 1129836$

Belsky, J., Crnic, K., \& Woodworth, S. (1995). Personality and parenting: Exploring the mediating role of transient mood and daily hassles. Journal of Personality, 63(4), 905929. doi: 10.1111/j.1467-6494.1995.tb00320.x

Berkovits, L. D., \& Baker, B. L. (2014). Emotion dysregulation and social competence: Stability, change and predictive power. Journal of Intellectual Disability Research, 58(8), 765-776. doi: $10.1111 /$ jir.12088

Betts, J., Gullone, E., \& Allen, J. S. (2009). An examination of emotion regulation, temperament, and parenting style as potential predictors of adolescent depression risk status: A correlational study. British Journal of Developmental Psychology, 27(2), 473-485. doi: $10.1348 / 026151008 \times 314900$

Blair, B. L., Perry, N. B., O’Brien, M., Calkins, S. D., Keane, S. P., \& Shanahan, L. (2014). The indirect effects of maternal emotion socialization on friendship quality in middle childhood. Developmental Psychology, 50(2), 566-576. doi: 10.1037/a0033532

Bowerman, B. L., \& O’Connell, R. T. (1990). Linear statistical models: An applied approach. ( $2^{\text {nd }}$ Ed.). Belmont, CA: Duxbury.

Brand, A. E., \& Klimes-Dougan, B. (2010). Emotion socialization in adolescence: The roles of 
mothers and fathers. In A. Kennedy Root \& S. Denham (Eds.), The role of gender in the socialization of emotion: Key concepts and critical issues (pp. 85-100). San Francisco: Jossey-Bass.

Breau, R. P., Harvey, E. A., Lugo-Candelas, C. I. (2016). The role of parent psychopathology in emotion socialization. Journal of Abnormal Child Psychology, 44(4), 731-743. doi: $10.1007 / \mathrm{s} 10802-015-0062-3$

Brumariu, L. E., \& Kerns, K. A. (2013). Pathways to anxiety: Contributions of attachment history, temperament, peer competence, and ability to manage intense emotions. Child Psychiatry \& Human Development, 44(4), 504-515. doi: 10.1007/s10578-012-0345-7

Buckholdt, K. E., Parra, G. R., \& Jobe-Shields, L. (2014). Intergenerational transmission of emotion dysregulation through parental invalidation of emotions: Implications for adolescent internalizing and externalizing behaviors. Journal of Child and Family Studies, 23(2), 324-332. doi: 10.1007/s10826-013-9768-4

Bugental, D. B. (1992). Affective and cognitive processes within threat-oriented family systems. In J. J. Goodnow (Ed.), Parental belief systems: The psychological consequences for children, $2^{\text {nd }}$ Ed. (pp. 219-248). Hillsdale, NJ: Lawrence Erlbaum Associates.

Bugental, D. B. (1987). Attributions as moderator variables within social interactional systems. Journal of Social and Clinical Psychology, 5(4), 469-484. doi: 10.1521/jscp.1987.5.4.469

Bugental, D. B., Blue, J., \& Lewis, J. (1990). Caregiver beliefs and dysphoric affect directed to difficult children. Developmental Psychology, 26(4), 631-638. 10.1037/00121649.26.4.631 
Bugental, D. B., \& Grusec, J. E. (2006). Socialization processes. In W. Damon \& R. Lerner (Eds.), Handbook of child psychology: Volume 3, social, emotional, and personality development, $6^{\text {th }}$ Ed. (pp. 366-428). Hoboken, NJ: Wiley.

Bugental, D. B., \& Happeney, K. (2002). Parental attributions. In M. H. Bornstein (Ed), Handbook of parenting: Vol. 3, being and becoming a parent, $2^{\text {nd }}$ Ed. (pp. 509-535). Mahwah, NJ: Lawrence Erlbaum Associates.

Bugental, D. B., \& Johnston C. (2000). Parental and child cognitions in the context of the family. Annual Review of Psychology, 51, 315-344. doi: 10.1146/annurev.psych.51.1.315

Bugental, D. B., Shennum, W. A., \& Shaver, P. (1984). "Difficult" children as elicitors and targets of adult communication patterns: An attributional-behavioral transactional analysis. Monographs of the Society for Research in Child Development, 49(1), 1-81. doi: $10.2307 / 1165910$

Bukowski, W. M., Brendgen, M., \& Vitaro, F. (2015). Peers and socialization: Effects on externalizing and internalizing problems. In J. E. Grusec \& P. D. Hastings (Eds.), Handbook of socialization: Theory and research, $2^{\text {nd }}$ Ed. (pp. 355-381). New York: Guilford Press.

Calkins, S. D., \& Dollar, J. M. (2014). Caregiving influences on emotion regulation: Educational implications of a biobehavioral perspective. In R. Pekrun \& L. Linnenbrink-Garcia (Eds.), International Handbook of emotions in education (pp. 520-538). New York: Routledge.

Campbell-Sills, L., Ellard, K. K., \& Barlow, D. H. (2014). Emotion regulation in anxiety disorders. In J. J. Gross (Ed.), Handbook of emotion regulation, $2^{\text {nd }}$ Ed. (pp. 393-412). New York: Guilford Press. 
Caprara, G. V. (2002). Personality psychology: Filling the gap between basic processes and molar functioning. In C. von Hofsten \& L. Backman (Eds.), Psychology at the turn of the millennium, Vol. 2: Social, developmental and clinical perspectives (pp. 201-224). New York: Psychology Press.

Caprara, G. V., \& Gerbino, M. (2001). The capacity to regulate negative affect and to express positive affect. In G. V. Caprara (Ed.), Self-efficacy assessment (pp. 35-50). Trento, Italy: Edizioni Erickson.

Caprara, G. V., Di Giunta, L., Eisenberg, N., Gerbino, M., Pastorelli, C., \& Tramontano, C. (2008). Assessing regulatory emotional self-efficacy in three countries. Psychological Assessment, 20(3), 227-237. doi: 10.1037/1040-3590.20.3.227

Caprara, G. V., Steca, P., Gerbino, M., Paciello, M., \& Vecchio, G. M. (2006). Looking for adolescents' well-being: Self-efficacy beliefs as determinants of positive thinking and happiness. Epidemiologia e Psichiatria Sociale, 15(1), 30-43. doi:

\subsection{7/S1121189X00002013}

Carlson, S. M., \& Wang, T. S. (2007). Inhibitory control and emotion regulation in preschool children. Cognitive Development, 22(4), 489-510. doi: 10.1016/j.cogdev.2007.08.002

Carver, C. S., \& Harmon-Jones, E. (2009). Anger is an approach-related affect: evidence and implications. Psychological Bulletin, 135(2), 183-204. doi: 10.1037/a0013965

Chan, S. M., Bowes, J., \& Wyver, S. (2009). Parenting style as a context for emotion socialization. Early Education and Development, 20(4), 631-656. doi:

$10.1080 / 10409280802541973$ 
Chen, X. (2000). Growing up in a collectivist culture: Socialization and socioemotional development in Chinese children. In H. Comunion \& V. Gielen (Eds.), International perspectives on human development (pp. 331-353). Padua, Italy: Cadam Publishers.

Chen, M., Johnston, C., Sheeber, L., \& Leve, C. (2009). Parent and adolescent depressive symptoms: The role of parental attributions. Journal of Abnormal Child Psychology, 37(1), 119-130. doi: 10.1007/s10802-008-9264-2

Cicchetti, D., Ackerman, B. P., \& Izard, C. E. (1995). Emotions and emotion regulation in developmental psychopathology. Development and Psychopathology, 7(1), 1-10. doi: $10.1017 / \mathrm{S} 0954579400006301$

Cohen, J. (1992). A power primer. Psychological Bulletin, 112(1), 155-159. doi: 10.1037/14805018

Cohen, J., Cohen, P., West, S. G., \& Aiken, L. S. (2003). Applied multiple regression/correlation analysis for the behavioral sciences. Mahwah, NJ: Lawrence Erlbaum Associates.

Cole, P. M., \& Tan, P. Z. (2015). Emotion socialization from a cultural perspective. In J. E. Grusec \& P. D. Hastings (Eds.). Handbook of socialization: Theory and research, $2^{\text {nd }}$ Ed. (pp. 499-519). New York: Guilford.

Cook, R. D., \& Weisberg, S. (1982). Residuals and influence in regression. New York: Chapman \& Hall.

Cooper, A., Gomez, R., \& Buck, E. (2008). The relationships between the BIS and BAS, anger and responses to anger. Personality and Individual Differences, 44(2), 403-413. doi: 10.1016/j.paid.2007.09.005 
Conger, R. D., \& Conger, K. J. (2002). Resilience in Midwestern families: Selected findings from the first decade of a prospective, longitudinal study. Journal of Marriage and Family, 64(2), 361-373. doi: 10.1111/j.1741-3737.2002.00361.x

Conger, R. D., Ge, X., Elder, G. H., Lorenz, F. O., \& Simons, R. L. (1994). Economic stress, coercive family process, and developmental problems of adolescents. Child Development, 65(2), 541-561. doi: 10.2307/1131401

Coplan, R. J., Hastings, P. D., Lagacé-Séguin, D. G., \& Moulton, C. E. (2002). Authoritative and authoritarian mothers' parenting goals, attributions, and emotions across different childrearing contexts. Parenting: Science and Practice, 2(1), 1-26. doi: 10.1207/S15327922PAR0201_1

Criss, M. M., Houltberg, B. J., Cui, L., Bosler, C. D., Sheffield Morris, A., \& Silk, J. S. (2016). Direct and indirect links between peer factors and adolescent adjustment difficulties. Journal of Applied Developmental Psychology, 43, 83-90. doi: 10.1016/j.appdev.2016.01.002

Crockenberg, S. B., \& Smith, P. (1982). Antecedents of mother-infant interaction and infant irritability in the first three months of life. Infant Behavior and Development, 5(1), 105 119. doi: 10.1016/S0163-6383(02)00088-7

Cui, L., Sheffield Morris, A., Criss, M. M., Houltberg, B. J., \& Silk, J. S. (2014). Parental psychological control and adolescent adjustment: The role of adolescent emotion regulation. Parenting: Science \& Practice, 14(1), 47-67. doi:

$10.1080 / 15295192.2014 .880018$

Dearing, E., \& Hamilton, L. C. (2006) Contemporary advances and classic advice for analyzing mediating and moderating variables. In K. McCartney, M. R. Burchinal, \& K. L. Bub 
(Eds.), Best practices in quantitative methods for developmentalists (pp. 1-145). United Kingdom: Blackwell Publishing.

Denham, S. A. (1993). Maternal emotional responsiveness and toddlers' social-emotional competence. Journal of Child Psychology and Psychiatry, 34(5), 715-728.

Denham, S. A., Bassett, H. H., \& Wyatt, T. (2015). The socialization of emotional competence. In J. E. Grusec \& P. D. Hastings (Eds.). Handbook of socialization: Theory and research: $2^{\text {nd }}$ Ed. (pp. 590-613). New York: Guilford.

Denham, S., \& Kochanoff, A. T. (2002). Parental contributions to preschoolers' understanding of emotion. Marriage \& Family Review, 34(3/4), 311-343. doi: 10.1300/J002v34n03_06

Dix, T. (1993). Attributing dispositions to children: An interactional analysis of attribution in socialization. Personality and Social Psychology Bulletin, 19(5), 633-643. doi: $10.1177 / 0146167293195014$

Dix, T. (1991). The affective organization of parenting: Adaptive and maladaptive processes. Psychological Bulletin, 110(1), 3-25. doi: 10.1037/0033-2909.110.1.3

Dix, T., \& Grusec, J. E. (1985). Parent attribution processes in the socialization of children. In I. E. Sigel (Ed.), Parental belief systems (pp. 201-233). Hillsdale, NJ: Lawrence Erlbaum Associates.

Dix, T., \& Lochman, J. E. (1990). Social cognition and negative reactions to children: A comparison of mothers of aggressive and nonaggressive boys. Journal of Social and Clinical Psychology, 9(4), 418-438. doi: 10.1521/jscp.1990.9.4.418

Dix, T., Ruble, D. N., Grusec, J. E., \& Nixon, S. (1986). Social cognition in parents: Inferential and affective reactions to children of three age levels. Child Development, 57(4), 879894. doi: $10.2307 / 1130365$ 
Dix, T., \& Reinhold, D. P. (1991). Chronic and temporary influences on mothers' attributions for children's disobedience. Merrill-Palmer Quarterly, 37(2), 251-271.

Dix, T., Reinhold, D. P., \& Zambarano, R. J. (1990). Mothers' judgments in moments of anger. Merrill-Palmer Quarterly, 36(4), 465-486.

Dix, T., Ruble, D. N., \& Zambarano, R. J. (1989). Mothers' implicit theories of discipline: Child effects, parent effects, and the attribution process. Child Development, 60(6), 1373-1391. doi: $10.2307 / 1130928$

Duchesne, S., Ratelle, C. F., Poitras, S.-C., \& Drouin, E. (2009). Early adolescent attachment to parents, emotional problems, and teacher-academic worries about the middle school transition. Journal of Early Adolescence, 29(5), 743-766.

Dweck, C. S. (2006). Mindset: The new psychology of success. New York: Random House.

Dweck, C. S. (1975). The role of expectations and attributions in the alleviation of learned helplessness. Journal of Personality and Social Psychology, 31(4), 674-685. doi: $10.1037 / \mathrm{h} 0077149$

Eccles, J. S. (1994). Schools, academic motivation, and stage-environment fit. In R. M. Lerner \& L. Steinberg (Eds.), Handbook of child psychology, $2^{\text {nd }}$ Ed. (pp. 125-153). Hoboken, New Jersey: Wiley.

Eccles, J. S., Midgley, C., Wigfield, A., Buchanan, C. M., Reuman, D., Flanagan, C., \& Mac Iver, D. (1993). Development during adolescence: The impact of stage-environment fit on young adolescents' experiences in schools and in families. American Psychologist, 48(2), 90-101. doi: 10.1037/10254-034

Eisenberg, N., Cumberland, A., \& Spinrad, T. L. (1998). Parental socialization of emotion. Psychological Inquiry, 9(4), 241-273. doi: 10.1207/s15327965pli0904_1 
Eisenberg, N., \& Fabes, R. A. (1994). Mothers' reactions to children's negative emotions: Relations to children's temperament and anger behavior. Merrill Palmer Quarterly, 40(1), 138-156.

Eisenberg, N., Fabes, R. A., \& Murphy, B. C. (1996). Parents' reactions to children's negative emotions: Relations to children's social competence and comforting behavior. Child Development, 67(5), 2227-2247.

Eisenberg, N., Hofer, C., \& Vaughan, J. (2007). Effortful control and its socioemotional consequences. In J. J. Gross (Ed.), Handbook of emotion regulation (pp. 287-306). New York: Guilford Press.

Eisenberg, N., Fabes, R. A., Shepard, S. A., Guthrie, I. K., Murphy, B. C., \& Reiser, M. (1999). Parental reactions to children's negative emotions: Longitudinal relations to quality of children's social functioning. Child Development, 70(2), 513-534. doi: 10.1111/14678624.00037

Eisenberg, N., \& Valiente, C. (2004). Elaborations on a theme: Beyond main effects in relations of parenting to children's coping and emotion regulation. Parenting: Science and Practice, 4(4), 319-323.doi: 10.1207/s15327922par0404_2

Fabes, R. A., Poulin, R. E., Eisenberg, N., \& Madden-Derdich, D. A. (2002). The Coping with Children's Negative Emotions Scale (CCNES): Psychometric properties and relations with children's emotional competence. Marriage and Family Review, 34(3/4), 285-310. doi: 10.1300/J002v34n03_05

Field, A. (2013). Discovering statistics using IBM SPSS statistics. Los Angeles: Sage Publications. 
Ford, B. Q., Mauss, I. B., Troy, A. S., Smolen, A., \& Hankin, B. (2014). Emotion regulation moderates the risk associated with the 5-HTT gene and stress in children. Emotion, 14(5), 930-939. doi: 10.1037/a0036835

Forrest, C. B., Bevans, K. B., Riley, A. W., Crespo, R., \& Louis, T. A. (2013). Health and school outcomes during children's transition into adolescence. Journal of Adolescent Health, 52(2), 186-194. doi: 10.1016/j.jadohealth.2012.06.019

Flett, G. L., Hewitt, P. L., Oliver, J. M., \& Macdonald, S. (2002). Perfectionism in children and their parents: A developmental analysis. In G. L. Flett \& P. L. Hewitt (Eds.), Perfectionism: Theory, research, and treatment (pp. 89-132). Washington, DC: American Psychological Association.

Flouri, E., \& Mavroveli, S. (2012). Adverse life events and emotional and behavioural problems in adolescence: The role of coping and emotion regulation. Stress and Health, 29(5), 360368. doi: $10.1002 / \mathrm{smi} .2478$

Fredrickson, B. L. (1998). Cultivated emotions: Parental socialization of positive emotions and self-conscious emotions. Psychological Inquiry, 9(4), 279-281. doi:

$10.1207 / \mathrm{s} 15327965$ pli0904_4

Friedlmeier, W., Corapci, F., \& Cole, P. M. (2011). Emotion socialization in cross-cultural perspective. Social and Personality Psychology Compass, 5(7), 410-427. doi: 10.1111/j.1751-9004.2011.00362.x

Garside, R. B., \& Klimes-Dougan, B. (2002). Socialization of discrete negative emotions: Gender differences and links with psychological distress. Sex Roles, 47(3/4), 115-128. doi: 10.1023/A:1021090904785 
Givertz, M., \& Segrin, C. (2012). The association between overinvolved parenting and young adults' self-efficacy, psychological entitlement, and family communication. Communication Research, 41(8), 1111-1136. doi: 10.1177/0093650212456392

Goodnow, J. J., Knight, R., \& Cashmore, J. (1986). Adult social cognition: Implications of parents' ideas for approaches to development. In M. Perlmutter (Ed.), Cognitive perspectives on children's social and behavioral development. Minnesota Symposium on Child Psychology, Vol. 18 (pp. 287-324). Hillsdale, NJ: Erlbaum.

Graber, J. A., \& Brooks-Gunn, J. (1996). Transitions and turning points: Navigating the passage from childhood through adolescence. Developmental Psychology, 32(4), 768-776. doi: $10.1037 / 0012-1649.32 .4 .768$

Granic, I., Hollenstein, T., Dishion, T. J., \& Patterson, G. R. (2003). Longitudinal analysis of flexibility and reorganization in early adolescence: A dynamic systems study of family interactions. Developmental Psychology, 39(3), 606-617. doi: 10.1037/00121649.39.3.606

Gottman, J. M., Katz, L. F., \& Hooven, C. (1996). Parental meta-emotion philosophy and the emotional life of families: Theoretical models and preliminary data. Journal of Family Psychology, 10(3), 243-268. doi: 10.1037/0893-3200.10.3.243

Graziano, P. A., Reavis, R. D., Keane, S. P., \& Calkins, S. D. (2007). The role of emotion regulation in children's early academic success. Journal of School Psychology, 45(1), 319. 10.1016/j.jsp.2006.09.002

Gretarsson, S. J., \& Gelfand, D. M. (1988). Mothers' attributions regarding their children's social behavior and personality characteristics. Developmental Psychology, 24(2), 264-269. doi: 10.1037/0012-1649.24.2.264 
Gross, J. J. (2002). Emotion regulation: Affective, cognitive, and social consequences. Psychophysiology, 39(3), 281-291. doi: 10.1017/S0048577201393198

Gross, J. J. (1998). Antecedent-and response-focused emotion regulation: divergent consequences for experience, expression, and physiology. Journal of Personality and Social Psychology, 74(1), 224-237. doi: 10.1037/0022-3514.74.1.224

Gross, J. J., \& John, O. P. (2003). Individual differences in two emotion regulation processes: implications for affect, relationships, and well-being. Journal of Personality and Social Psychology, 85(2), 348-362. doi: 10.1037/0022-3514.85.2.348

Grusec, J. E., \& Davidov, M. (2015). Analyzing socialization from a domain-specific perspective. In J. E. Grusec \& P. D. Hastings (Eds.), Handbook of socialization: Theory and research, $2^{\text {nd }}$ Ed. (pp. 158-181). New York: Guilford Press.

Grusec, J. E., Adam, E., \& Mammone, N. (1993, April). Mental representations of relationships, parent belief systems, and parenting behavior. Paper presented at the biennial meeting of the Society for Research in Child Development, New Orleans, LA.

Grusec, J. E., Hastings, P. D., \& Mammone (1994). Parenting cognitions and relationship schemas. New Directions for Child Development, 66, 5-18.

Gumora, G., \& Arsenio, W. F. (2002). Emotionality, emotion regulation, and school performance in middle school children. Journal of School Psychology, 40(5), 395-413. doi: 10.1016/S0022-4405(02)00108-5

Gullone, E., \& Taffe, J. (2012). The Emotion Regulation Questionnaire for Children and Adolescents (ERQ-CA): A psychometric evaluation. Psychological Assessment, 24(2), 409-417. doi: 10.1037/a0025777 
Gunzenhauser, C., Fäsche, A., Friedlmeier, W., \& von Suchodoletz, A. (2014). Face it or hide it: Parental socialization of reappraisal and response suppression. Frontiers in Psychology, 4, 1-14. doi: 10.3389/fpsyg.2013.00992

Guo, J., Mrug, S., \& Knight, D. C. (2016). Factor structure of the emotions as a child scale in late adolescence and emerging adulthood. Psychological Assessment. Advance online publication. doi: 10.1037/pas0000412

Hastings, P. D., Klimes-Dougan, B., Kendziora, K. T., Brand, A., \& Zahn-Waxler, C. (2014). Regulating sadness and fear from outside and within: Mothers' emotion socialization and adolescents' parasympathetic regulation predict the development of internalizing difficulties. Development and Psychopathology, 26(4), 1369-1384. doi:

$10.1017 / \mathrm{S} 0954579414001084$

Hastings, P. D., \& Rubin, K. H. (1999). Predicting mothers' beliefs about preschool aged children's social behavior: Evidence for maternal attitudes moderating child effects. Developmental Psychology, 70(3), 722-741. doi: 10.1111/1467-8624.00052

Heatherington, L., McDonald, M., Tolejko, N., \& Funk, J. (2007). Now why'd he do that? The nature and correlates of mothers' attributions about teen behavior. Journal of Family Psychology, 21(2), 315-319. doi: 10.1037/0893-3200.21.2.315

Hollenstein, T., \& Lougheed, J. P. (2013). Beyond storm and stress: Typicality, transactions, timing, and temperament to account for adolescent change. American Psychologist, 68(6), 444-454. doi: 10.1037/a0033586

Hu, T., Zhang, D., Wang, J., Mistry, R., Ran, G., \& Wang, X. (2014). Relation between emotion regulation and mental health: A meta-analysis review. Psychological Reports: Measures and Statistics, 114(2), 341-362. doi: 10.2466/03.20.PR0.114k22w4 
Hudson, A., \& Jacques, S. (2014). Put on a happy face! Inhibitory control and socioemotional knowledge predict emotion regulation in 5- to 7-year-olds. Journal of Experimental Child Psychology, 123, 36-52. doi: 10.1016/j.jecp.2014.01.012

Hurrell, K. E., Hudson, J. L., \& Schniering, C. A. (2015). Parental reactions to children's negative emotions: Relationships with emotion regulation in children with an anxiety disorder. Journal of Anxiety Disorders, 29, 72-82. doi: 10.1016/j.janxdis.2014.10.008

Huebner, R. R., \& Izard, C. E. (1988). Mothers’ responses to infants’ facial expressions of sadness, anger, and physical distress. Motivation and Emotion, 12(2), 185-196. doi: 10.1007/BF00992173

Izard, C. E., \& Ackerman, B. P. (2000). Organizational and motivational functions of discrete emotions. In M. Lewis, J.M. Haviland-Jones, \& L. F. Barrett (Eds.), Handbook of emotions, $2^{\text {nd }}$ ed. (pp. 253-264). New York: Guilford Press.

Izard, C. E., (1978). On the development of emotions and emotion-cognition relationships in infancy. In M. Lewis \& L. A. Rosenblum (Eds.), The development of affect (pp. 389413). New York: Plenum Press.

Izard, C., Fine, S., Schultz, D., Mostow, A., Ackerman, B., \& Youngstrom, E. (2001). Emotion knowledge as a predictor of social behavior and academic competence in children at risk. Psychological Science, 12(1), 18-23. doi: 10.1111/1467-9280.00304

Jacob, M. L., Thomassin, K., Morelen, D., \& Suveg, C. (2011). Emotion regulation in childhood anxiety. In D. McKay \& E. A. Storch (Eds.), Handbook of child and adolescent anxiety disorders (pp. 171-185). New York: Springer. 
John, O. P., \& Gross, J. J. (2004). Healthy and unhealthy emotion regulation: Personality processes, individual differences, and life span development. Journal of Personality, 72(6), 1301-1334. doi: 10.1111/j.1467-6494.2004.00298.x

Johnston, C., \& Freeman, W. (1997). Attributions for child behavior in parents of children without behavior disorders and children with attention deficit-hyperactivity disorder. Journal of Consulting and Clinical Psychology, 65(4), 636-645. doi: 10.1037/0022-006X.65.4.636

Jones, D. C., Abbey, B. B., \& Cumberland, A. (1998). The development of display rule knowledge: Linkages with family expressiveness and social competence. Child Development, 69(4), 1209-1222. doi: 10.2307/1132370

Joormann, J., \& Vanderlind, W. M. (2014). Emotion regulation in depression: The role of cognition and reduced cognitive control. Clinical Psychological Science, 2(4), 402-421. doi: $10.1177 / 2167702614536163$

Kahle, S., Utendale, W. T., Widaman, K. F., \& Hastings, P. D. (2017). Parasympathetic regulation and inhibitory control predict the development of externalizing problems in early childhood. Journal of Abnormal Child Psychology. Advance online publication. doi: $10.1007 /$ s10802-017-0305-6

Kail, R. (1993). Processing time decreases globally at an exponential rate during childhood and adolescence. Journal of Experimental Child Psychology, 56(2), 254-265. doi: 10.1006/jecp.1993.1034

Kansky, J., Allen, J. P., \& Diener, E. (2016). Early adolescent affect predicts later life outcomes. Applied Psychology: Health and Well-Being, 8(2), 192-212. doi: 10.1111/aphw.12068 
Katz, L. F., Shortt, J. W., Allen, N. B., Davis, B., Hunter, E., Leve, C., \& Sheeber, L. (2014). Parental emotion socialization in clinically depressed adolescents: Enhancing and dampening positive affect. Journal of Abnormal Child Psychology, 42(2), 205-215. doi: $10.1007 / \mathrm{s} 10802-013-9784-2$

Kelley, S. A., Brownell, C. A., \& Campbell, S. B. (2000). Mastery motivation and selfevaluative affect in toddlers: Longitudinal relations with maternal behavior. Child Development, 71(4), 1061-1071. doi: 10.1111/1467-8624.00209

Kennedy, A. E., Rubin, K. H., Hastings, P., \& Maisel, B. (2004). Longitudinal relations between child vagal tone and parenting behavior: 2 to 4 years. Developmental Psychobiology, 45(1), 10-21. doi: 10.1002/dev.20013

Kim, J., \& Cicchetti, D. (2010). Longitudinal pathways linking child maltreatment, emotion regulation, peer relations, and psychopathology. Journal of Child Psychology and Psychiatry, 51(6), 706-716. doi: 10.1111/j.1469-7610.2009.02202.x

Klimes-Dougan, B., Brand, A. E., Zahn-Waxler, C., Usher, B., Hastings, P. D., Kendziora, K., \& Garside, R. B. (2007). Parental emotion socialization in adolescence: Differences in age, sex, and problem status. Social Development, 16(2), 326-342. doi: 10.1111/j.14679507.2007.00387.x

Kolubinski, D. C., Nikcevic, A. V., Lawrence, J. A., \& Spada, M. M. (2016). The role of metacognition in self-critical rumination: An investigation in individuals presenting with low self-esteem. Journal of Rational-Emotive Cognitive Behavior Therapy, 34(1), 73-85. doi: 10.1007/s10942-015-0230-y 
Kuczynski, L., \& Hildebrant, N. (1997). Models of conformity and resistance in socialization theory. In J. E. Grusec \& L. Kuczynski (Eds.), Parenting and the internalization of values: A handbook of contemporary theory (pp. 227-256). New York: Wiley.

Kuczynski, L., \& Parkin, C. M. (2009). Pursuing a dialectical perspective on transaction: A social relational theory of micro family processes. In A. Sameroff (Ed.), The transactional model of development: How children and contexts shape each other (pp. 247-268). Washington, DC: American Psychological Association.

Kuczynski, L., \& Parkin, C. M. (2007). Agency and bidirectionality in socialization: Interactions, transactions, and relational dialectics. In J. E. Grusec \& P. D. Hastings (Eds.), Handbook of socialization: Theory and research (pp. 259-283). New York: Guilford Press.

Kuczynski, L., Harach, L., \& Bernadini, S. C. (1999). Psychology’s child meets sociology’s child: Agency, power, and influence in parent-child relations. In C. Shehan (Ed.), Through the eyes of a child: Revisioning children as active agents of family life (pp. 2152). Stamford, CT: JAI Press.

Labouvie-Vief, G., DeVoe, M., \& Bulka, D. (1989). Speaking about feelings: Conceptions of emotion across the lifespan. Psychology and Aging, 4(4), 425-437. doi: 10.1037/08827974.4.4.425

Laukkanen, J., Ojansuu, U., Tolvanen, A., Alatupa, S., \& Aunola, K. (2014). Child's difficult temperament and mothers' parenting styles. Journal of Child and Family Studies, 23(2), 312-323. doi: 10.1007/s10826-013-9747-9

Lemerise, E. A., \& Dodge, K. A. (2008). The development of anger and hostile interactions. In M. Lewis, J.M. Haviland-Jones, \& L. F. Barrett (Eds.), Handbook of emotions (pp. 730741). New York: Guilford Press. 
Levitt, M. J., Levitt, J., Bustos, G. L., Crooks, N. A., Santos, J. D., Telan, P., ... \& Milevsky, A. (2005). Patterns of social support in the middle childhood to early adolescent transition: Implications for adjustment. Social Development, 14(3), 398-420. doi: 10.1111/j.14679507.2005.00308.x

Lewis, M. (2008). Self-conscious emotions: Embarrassment, pride, shame, and guilt. In M. Lewis, J.M. Haviland-Jones, \& L. F. Barrett (Eds.), Handbook of emotions (pp. 742-756). New York: Guilford Press.

Liew, J., Eisenberg, N., Losoya, S. H., Guthrie, I. K., \& Murphy, B. C. (2003). Children's physiological indices of empathy and their socioemotional adjustment: Does caregivers' expressivity matter? Journal of Family Psychology, 17(4), 584-597. doi: 10.1037/08933200.17 .4 .584

Lippold, M. A., Powers, C. J., Syvertsen, A. K., Feinberg, M. E., \& Greenberg, M. T. (2012). The timing of school transitions and early adolescent problem behavior. The Journal of Early Adolescence, 33(6), 821-844. doi: 10.1177/0272431612468317

Lipscomb, S. T., Leve, L. D., Harold, G. T., Neiderhiser, J. M., Shaw, D. S., Ge, X., \& Reiss, D. (2011). Trajectories of parenting and child negative emotionality during infancy and toddlerhood: A longitudinal analysis. Child Development, 82(5), 1661-1675. doi: 10.1111/j.1467-8624.2011.01639.x

Lopes, P. N., Mestre, J. M., Guil, R., Kremenitzer, J. P., \& Salovey, P. (2012). The role of knowledge and skills for managing emotions in adaptation to school: Social behavior and misconduct in the classroom. American Educational Research Journal, 49(4), 710-742. doi: $10.3102 / 0002831212443077$ 
Luebbe, A. M., Kiel, E. J., \& Buss, K. A. (2011). Toddlers' context-varying emotions, maternal responses to emotions, and internalizing behaviors. Emotion, 11(3), 697-703. doi: $10.1037 / \mathrm{a} 0022994$

Neppl, T. K., Senia, J. M., \& Donnellan, M. B. (2016). Effects of economic hardship: Testing the family stress model over time. Journal of Family Psychology, 30(1), 12-21. doi: 10.1037/fam0000168

Nolen-Hoeksema, S. (2012). Emotion regulation and psychopathology: The role of gender. Annual Review of Clinical Psychology, 8, 161-187. doi: 10.1146/annurevclinpsy-032511-143109

Maccoby, E. E. (2015). Historical overview of socialization research and theory. In J. E. Grusec \& P. D. Hastings (Eds.), Handbook of socialization: Theory and research, $2^{\text {nd }}$ Ed. (pp. 30-34). New York: Guilford Press.

Magai, C. (1996). Emotions as a child. Unpublished manuscript, Long Island University, Brooklyn, New York.

Malatesta, C. Z., \& Haviland, J. M. (1982). Learning display rules: The socialization of emotion expression in infancy. Child Development, 53(4), 991-1003.

Mathieu, J. E., \& Taylor, S. R. (2006). Clarifying conditions and decision points for mediational type inferences in organizational behavior. Journal of Organizational Behavior, 27(8), 1031-1056. doi: 10.1002/job.406

Mennin, D. S., Fresco, D. M., Ritter, M., \& Heimberg, R. G. (2015). An open trial of emotion regulation therapy for generalized anxiety disorder and co-occurring depression. Depression and Anxiety, 32(8), 614-623. doi: 10.1002/da.22377 
Midgley, C., Eccles, J. S., \& Feldlaufer, H. (1988). Classroom environment and the transition to junior high school. In B. J. Fraser \& H. J. Walberg (Eds.), Educational environments: Evaluation, antecedents, and consequences (pp. 13-139). Elmsford, NY: Pergamon Press.

Miller, S. A. (1995). Parents' attributions for their children's behavior. Child Development, 66(6), 1557-1584. doi: 10.2307/1131897

Miller, S. A., Hardin, C. A., \& Montgomery, D. E. (2003). Young children's understanding of the conditions for knowledge acquisition. Journal of Cognition and Development, 4(3), 325356. doi: 10.1207/S15327647JCD0403_05

Mills, R. S. L. (1998). Paradoxical relations between perceived power and maternal control. Merrill-Palmer Quarterly, 44(4), 523-537.

Mills, R. S. L., \& Rubin, K. H. (1992). A longitudinal study of maternal beliefs about children's social behaviors. Merrill-Palmer Quarterly, 38(4), 494-512.

Mills, R. S. L., \& Rubin, K. H. (1990). Parental beliefs about problematic social behaviors in early childhood. Child Development, 61(1), 138-151. doi: 10.2307/1131054

Monopoli, W. J., \& Kingston, S. (2012). The relationships among language ability, emotion regulation and social competence in second-grade students. International Journal of Behavioral Development, 36(5), 398-405. doi: 10.1177/0165025412446394

Nader-Grosbois, N. (2014). Self-perception, self-regulation and metacognition in adolescents with intellectual disability. Research in Developmental Disabilities, 35(6), 334-1348. doi: 10.1016/j.ridd.2014.03.033

Nelson J. A., O’Brien M., Blankson A.N., Calkins, S. D., \& Keane, S.P. (2009) Family stress and parental responses to children's negative emotions: Tests of the spillover, crossover, 
and compensatory hypotheses. Journal of Family Psychology, 23(5), 671-679. doi: $10.1037 / \mathrm{a} 0015977$

Nelson, J. A., O’Brien, M., Calkins, S. D., \& Keane, S. P. (2013). Mothers' and fathers' negative responsibility attributions of children's problem behavior. Personal Relationships, 20(4), 719-727. doi: 10.1111/pere. 12010

Öhman, A. (2008). Fear and anxiety: Overlaps and dissociations. In M. Lewis, J.M. HavilandJones, \& L. F. Barrett (Eds.), Handbook of emotions (pp. 709-729). New York: Guilford Press.

O’Keeffe, G. S., \& Clarke-Pearson, K. (2011). The impact of social media on children, adolescents, and families. Pediatrics, 127(4), 800-804. doi: 10.1542/peds.2011-0054

Olson, S. L., Schilling, E. M., \& Bates, J. E. (1998). Measurement of impulsivity: Construct coherence, longitudinal stability, and relationship with externalizing problems in middle childhood and adolescence. Journal of Abnormal Child Psychology, 27(2), 151-165. doi: 10.1023/A:1021915615677

O’Neal, C. R., \& Magai, C. (2005). Do parents respond in different ways when children feel different emotions? The emotional context of parenting. Development and Psychopathology, 17(2), 467-487. doi: 10.1017/S0954579405050224

Paquette, D. (2004). Theorizing the father-child relationship: Mechanisms and developmental outcomes. Human Development, 47(4), 193-219. doi: 10.1159/000078723

Parke, R. D. (1994). Progress, paradigms, and unresolved problems: A commentary on recent advances in our understanding of children's emotions. Merrill-Palmer Quarterly, 40(1), 157-169. 
Paulussen-Hoogeboom, M. C., Stams, G. J. J., Hermanns, J. M., \& Peetsma, T. T. (2008).

Relations among child negative emotionality, parenting stress, and maternal sensitive responsiveness in early childhood. Parenting: Science and Practice, 8(1), 1-16. doi:

$10.1080 / 15295190701830656$

Porter, C. L., Hart, C. H., Yang, C., Robinson, C. C., Olsen, S. F., Zeng, Q., \& Olsen, J. A. (2005). A comparative study of child temperament and parenting in Beijing, China and the western United States. International Journal of Behavioral Development, 29(6), 541551. doi: 10.1080/01650250500147402

Pianta, R. C., Hamre, B., \& Stuhlman, M. (2003). Relationships between teachers and children. In W. M. Reynolds \& G. E. Miller (Eds.), Handbook of child psychology: Educational psychology, vol. 7 (pp. 199-234). Hoboken, New Jersey: Wiley.

Pratto, F., \& John, O. P. (1991). Automatic vigilance: The attention-grabbing power of negative social information. Journal of Personality and Social Psychology, 61(3), 380-391. doi: $10.1037 / 0022-3514.61 .3 .380$

Preacher, K. J., \& Hayes, A. F. (2004). SPSS and SAS procedures for estimating indirect effects in simple mediation models. Behavior Research Methods, Instruments, and Computers, 36(4), 717-731. doi: 10.3758/BF03206553

Prot, S., Anderson, C. A., Gentile, D. A., Warburton, W., Saleem, M., Groves, C. L., \& Brown, S. C. (2015). Media as agents of socialization. In J. E. Grusec \& P. D. Hastings (Eds.), Handbook of socialization: Theory and research, $2^{\text {nd }}$ Ed. (pp. 276-300). New York: Guilford Press. 
Puff, J., \& Renk, K. (2016). Mothers' temperament and personality: Their relationship to parenting behaviors, locus of control, and young children's functioning. Child Psychiatry and Human Development, 47(5), 799-819. doi: 10.1007/s10578-015-0613-4

Rasmussen, K. E. (2015). Adaptation of the Regulatory Emotional Self-Efficacy (RESE) scale for children. Unpublished manuscript.

Rasmussen, K. E., Hartman, S. W., \& Root, A. E. (2017, April). Running on empty: The effect of chronic economic hardship on three parenting styles in adolescence. Poster presented at the Biennial Meeting of the Society for Research in Child Development, Austin, TX.

Raval, V. V., \& Martini, T. S. (2011). "Making the child understand:" Socialization of emotion in urban India. Journal of Family Psychology, 25(6), 847-856. doi: 10.1037/a0025240

Raval, V. V., Daga, S. S., Raval, P. H., \& Panchal, I. N. (2016). Asian Indian mothers' emotion socialization and child emotion expression as a function of situational context. Journal of Child and Family Studies, 25(9), 2853-2861. doi: 10.1007/s10826-016-0451-4

Rawana, J. S., Flett, G. L., McPhie, M. L., Nguyen, H. T., \& Norwood, S. J. (2014). Developmental trends in emotion regulation: A systematic review with implications for community mental health. Canadian Journal of Community Mental Health, 33(1), 31-44. doi: $10.7870 / \mathrm{cjcmh}-2014-004$

Riediger, M., \& Klipker, K. (2014). Emotion regulation in adolescence. In J. J. Gross (Ed.), Handbook of emotion regulation (pp. 187-202). New York: Guilford Press.

Roeser, R. W., \& Eccles, J. S. (1998). Adolescents' perceptions of middle school: Relation to longitudinal changes in academic and psychological adjustment. Journal of Research on Adolescence, 8(1), 123-158. doi: 10.1207/s15327795jra0801_6 
Root, A. E., \& Rasmussen, K. E. (2017). Maternal emotion socialization: The contribution of inhibited behaviour and mothers' dissatisfaction with the parent-child relationship. Infant and Child Development, 26(1), 1-12. doi: 10.1002/icd.1955

Root, A. K., \& Stifter, C. (2010). Temperament and maternal emotion socialization beliefs as predictors of early childhood social behavior in the laboratory and classroom. Parenting: Science and Practice, 10(4), 241-257. doi: 10.1080/15295192.2010.492035

Rothbart, M. K., \& Bates, J. E. (2006). Temperament. In W. Damon (Editor-in-Chief), N. Eisenberg, \& R. M. Lerner (Volume Eds.), Handbook of child psychology: Vol. 3 Social, emotional, and personality development, $6^{\text {th }}$ Ed. (pp. 99-166). New York: Wiley.

Rothbart, M. K., Sheese, B. E., \& Posner, M. I. (2014). Temperament and emotion regulation. In J. J. Gross (Ed.), Handbook of emotion regulation, $2^{\text {nd }}$ Ed. (pp. 305-320). New York: Guilford Press.

Rubin, K. H.; Bukowski, W., \& Parker, J. G. (1998). Peer interactions, relationships, and groups. In W. Damon \& N. Eisenberg (Eds.), Handbook of child psychology: Vol. 3. social, emotional, and personality development, 5th Ed. (pp. 619-700). New York: Wiley.

Rydell, A. M., Thorell, L. B., \& Bohlin, G. (2007). Emotion regulation in relation to social functioning: An investigation of child self-reports. European Journal of Developmental Psychology, 4(3), 293-313. doi: 10.1080/17405620600783526

Saarni, C. (1993). Socialization of emotion. In M. Lewis \& J. M. Haviland (Eds.), Handbook of Emotions (pp. 435-446). New York: Guilford Press.

Saarni, C. (1988). Children's understanding of the interpersonal consequences of dissemblance of nonverbal emotional-expressive behavior. Journal of Nonverbal Behavior, 12(4), 275294. doi: 10.1007/BF00987596 
Saarni, C. (1985). Indirect processes in affect socialization. In M. Lewis \& C. Saarni (Eds.), The socialization of emotions (pp. 187-209). New York: Plenum.

Saarni, C., \& Crowley, M. (1990). The development of emotion regulation: Effects on emotional state and expression. In E. A. Blechman (Ed.), Emotions and the family: For better or for worse (pp. 53-73). Hillsdale, New Jersey: Lawrence Erlbaum Associates.

Sameroff, A. J. (1975). Early influences on development: Fact or fancy? Merrill-Palmer Quarterly of Behavior and Development, 21(4), 267-294.

Scaramella, L. V., \& Leve, L. D. (2004). Clarifying parent-child reciprocities during early childhood: The early childhood coercion model. Clinical Child and Family Psychology Review, 7(2), 89-107. doi: 10.1023/B:CCFP.0000030287.13160.a3

Schachar, R., \& Logan, G. D. (1990). Impulsivity and inhibitory control in normal development and childhood psychopathology. Developmental Psychology, 26(5), 710-720. doi: 10.1037/0012-1649.26.5.710

Seidman, E., Allen, L., Aber, J. L., Mitchell, C., \& Feinman, J. (1994). The impact of school transitions in early adolescence on the self-system and perceived social context of poor urban youth. Child Development, 65(2), 507-522.

Sessa, F. M., Avenevoli, S., Steinberg, L., \& Morris, A. S. (2001). Correspondence among informants on parenting: Preschool children, mothers, and observers. Journal of Family Psychology, 15(1), 53-68. doi: 10.1037/0893-3200.15.1.53

Sheeber, L. B., Johnston, C., Chen, M., Leve, C., Hops, H., \& Davis, B. (2009). Mothers' and fathers' attributions for adolescent behavior: An examination in families of depressed, subdiagnostic, and nondepressed youth. Journal of Family Psychology, 23(6), 871-881. doi: $10.1037 / \mathrm{a} 0016758$ 
Sheffield Morris, A., Silk, J. S., Steinberg, L., Myers, S. S., \& Robinson, L. R. (2007). The role of the family context in the development of emotion regulation. Social Development, 16(2), 361-388. doi: 10.1111/j.1467-9507.2007.00389.x

Shewark, E. A., \& Blandon, A. Y. (2015). Mothers' and fathers' emotion socialization and children's emotion regulation: A within-family model. Social Development, 24(2), 266284. doi: 10.1111/sode. 12095

Shields, A., \& Cicchetti, D. (1997). Emotion regulation among school-age children: the development and validation of a new criterion Q-sort scale. Developmental Psychology, 33(6), 906-916. doi: 10.1037/0012-1649.33.6.906

Shields, A. M., \& Cicchetti, D. (1995, March). The development of an emotion regulation assessment battery: Reliability and validity among at-risk grade-school children. Poster session presented at the biennial meeting of the Society for Research in Child Development, Indianapolis, IN.

Silk, J. S., Shaw, D. S., Forbes, E. E., Lane, T. L., \& Kovacs, M. (2006). Maternal depression and child internalizing: The moderating role of child emotion regulation. Journal of Clinical Child and Adolescent Psychology, 35(1), 116-126. doi: $10.1207 / \mathrm{s} 15374424 \mathrm{jccp} 3501 \_10$

Silk, J. S., Shaw, D. S., Prout, J. T., O' Rourke, F., Lane, T. J., \& Kovacs, M. (2011). Socialization of emotion and offspring internalizing symptoms in mothers with childhood-onset depression. Journal of Applied Developmental Psychology, 32(3), 127136. doi: 10.1016/j.appdev.2011.02.001 
Slep, A. M. S., \& O’Leary, S. G. (1998). The effects of maternal attributions on parenting: An experimental analysis. Journal of Family Psychology, 12(2), 234-243. doi: 10.1037/08933200.12 .2 .234

Smetana, J. G., Robinson, J., \& Rote, W. M. (2015). Socialization in adolescence. In J. E. Grusec \& P. D. Hastings (Eds.), Handbook of socialization: Theory and research, $2^{\text {nd }}$ Ed. (pp. 60-84). New York: Guilford Press.

Smiley, P. A., Buttitta, K. V., Chung, S. Y., Coffey, J. K., Wang, B. A., \& Borelli, J. L. (2016). Anger in response to challenge: children's emotion socialization predicts approach versus avoidance. Motivation and Emotion, 40(6), 923-935. doi: 10.1007/s11031-016-9583-5

Smits, D. J. M., \& Kuppens, P. (2005). The relations between anger, coping with anger, and aggression, and the BIS/BAS system. Personality and Individual Differences, 39(4), 783793. doi: 10.1016/j.paid.2005.02.023

Spinrad, T. L, Eisenberg, N., Gaertner, B., Popp, T., Smith, C. L., Kupfer, A., . . Hofer, C. (2007). Relations of maternal emotion socialization and toddlers' effortful control to children's social adjustment and social competence. Developmental Psychology, 43(5), 1170-1186. doi: 10.1037/0012-1649.43.5.1170

Sundermann, J. M., \& DePrince, A. P. (2015). Maltreatment characteristics and emotion regulation (ER) difficulties as predictors of mental health symptoms: Results from a community-recruited sample of female adolescents. Journal of Family Violence, 30(3), 329-338. doi: 10.1007/s10896-014-9656-8

Suveg, C., Zeman, J., Flannery-Schroeder, E., \& Cassano, M. (2005). Emotion socialization in families of children with an anxiety disorder. Journal of Abnormal Child Psychology, 33(2), 145-155. doi: 10.1007/s10802-005-1823-1 
Tabibi, Z., \& Pfeffer, K. (2007). Finding a safe place to cross the road: The effect of distractors and the role of attention in children's identification of safe and dangerous road-crossing sites. Infant and Child Development, 16(2), 193-206. doi: 10.1002/icd.509

Taylor, Z. E., Eisenberg, N., Spinrad, T. L., Eggum, N. D., \& Sulik, M. J. (2013). The relations of ego-resiliency and emotion socialization to the development of empathy and prosocial behavior across early childhood. Emotion, 13(5), 822-831. doi: 10.1037/a0032894

Thomas, A., \& Chess, S. (1977). Temperament and development. Oxford: Brunner.

Uhls, Y. T., \& Greenfield, P. M. (2012). The value of fame: Preadolescent perceptions of popular media and their relationship to future aspirations. Developmental Psychology, $48(2), 315-326$.

Underwood, M. K. (1997). Peer social status and children's understanding of the expression and control of positive and negative emotions. Merrill-Palmer Quarterly, 43(4), 610-634.

Utendale, W. T., \& Hastings, P. D. (2010). Developmental changes in the relations between inhibitory control and externalizing problems during early childhood. Infant and Child Development, 20(2), 181-193. doi: 10.1002/icd.691

van der Bruggen, C. O., Stams, G. J. J., Bögels, S. M., \& Paulussen-Hoogeboom, M. C. (2010). Parenting behaviour as a mediator between young children's negative emotionality and their anxiety/depression. Infant and Child Development, 19(4), 354-365. doi: $10.1002 /$ icd. 665

Walsh, F. (2012). Clinical views of family normal- ity, health, and dysfunction: From a de cits to a strengths perspective. In F. Walsh (Ed.), Normal family processes (pp. 28-54). New York: Guilford Press. 
Wang, Q. (2013). Chinese socialization and emotion talk between mothers and children in native and immigrant Chinese families. Asian American Journal of Psychology, 4(3), 185-192. doi: $10.1037 / \mathrm{a} 0030868$

Warren, H. K., \& Stifter, C. A. (2008). Maternal emotion-related socialization and preschoolers' developing emotion self-awareness. Social Development, 17(2), 239-258. doi: 10.1111/j.1467-9507.2007.00423.x

Weiner, B. (1985). An attributional theory of achievement motivation and emotion. Psychological Review, 92(4), 548-573. doi: 10.1037/0033-295X.92.4.548

Weiner, B., Frieze, I. H., Kukla, A., Reed, L., Rest, S., \& Rosenbaum, R. M. (1971). Perceiving the causes of success and failure. Morristown, NJ: General Learning Press.

Welling, H. (2003). An evolutionary function of the depressive reaction: The cognitive map hypothesis. New Ideas in Psychology, 21(2), 147-156. doi: 10.1016/S0732$118 \mathrm{X}(03) 00017-5$

Wentzel, K. R., \& Looney, L. (2015). Socialization in school settings. In J. E. Grusec \& P. D. Hastings (Eds.), Handbook of socialization: Theory and research, $2^{\text {nd }}$ Ed. (pp. 382-403). New York: Guilford Press.

Whittingham, K., Sofronoff, K., Sheffield, K., \& Sanders, M. R. (2008). An exploration of parental attributions within the autism spectrum disorders population. Behaviour Change, 25(4), 201-214. doi: 10.1375/bech.25.4.201

Wilkins, A. C., \& Pace, J. A. (2014). Class, race, and emotions. In J. E. Stets \& J. H. Turner (Eds.), Handbook of the sociology of emotions: Volume II (pp. 385-409). Netherlands: Springer. 
Whiteman, M., Fanshel, D., \& Grundy, J. F. (1987). Cognitive-behavioral interventions aimed at anger of parents at risk of child abuse. Social Work, 32(6), 469-474.

Yap, M. B. H., Allen, N. B., \& Ladouceur, C. D. (2008). Maternal socialization of positive affect: The impact of invalidation on adolescent emotion regulation and depressive symptomology. Child Development, 79(5), 1415-1431. doi: 10.1111/j.14678624.2008.01196.x

Yan, J., Han, Z. R., \& Li, P. (2016). Intergenerational transmission of perceived bonding styles and paternal emotion socialization: Mediation through paternal emotion dysregulation. Journal of Child and Family Studies, 25(1), 165-175. doi: 10.1007/s10826-015-0199-2

Yi, C. I., Gentzler, A. L., Ramsey, M. A., \& Root, A. E. (2016). Linking maternal socialization of positive emotions to children's behavioral problems: The moderating role of selfcontrol. Journal of Child and Family Studies, 25(5), 1550-1558. doi: 10.1007/s10826015-0329-x

Zeman, J., \& Garber, J. (1996). Display rules for anger, sadness, and pain: It depends on who is watching. Child Development, 67(3), 957-973. doi: 10.2307/1131873

Zeman, J., \& Shipman, K. (1997). Social-contextual influences on expectancies for managing anger and sadness: the transition from middle childhood to adolescence. Developmental Psychology, 33(6), 917-924. doi: 10.1037/0012-1649.33.6.917

Zeman, J., \& Shipman, K. (1996). Children's expression of negative affect: Reasons and methods. Developmental Psychology, 32(5), 842-849. doi: 10.1037/0012-1649.32.5.842

Zeman, J., Perry-Parrish, C., \& Cassano, M. (2010). Parent-child discussions of anger and sadness: The importance of parent and child gender during middle childhood. In A. K. 
Root \& S. A. Denham (Eds.), Focus on gender: Parent and child contributions to the socialization of emotional competence (pp. 65-83). San Francisco, CA: Jossey-Bass.

Zimmer-Gembeck, M. J., \& Skinner, E. A. (2011). The development of coping across childhood and adolescence: An integrative review and critique of research. International Journal of Behavioural Development, 35(1), 1-17. doi: 10.1177/0165025410384923 

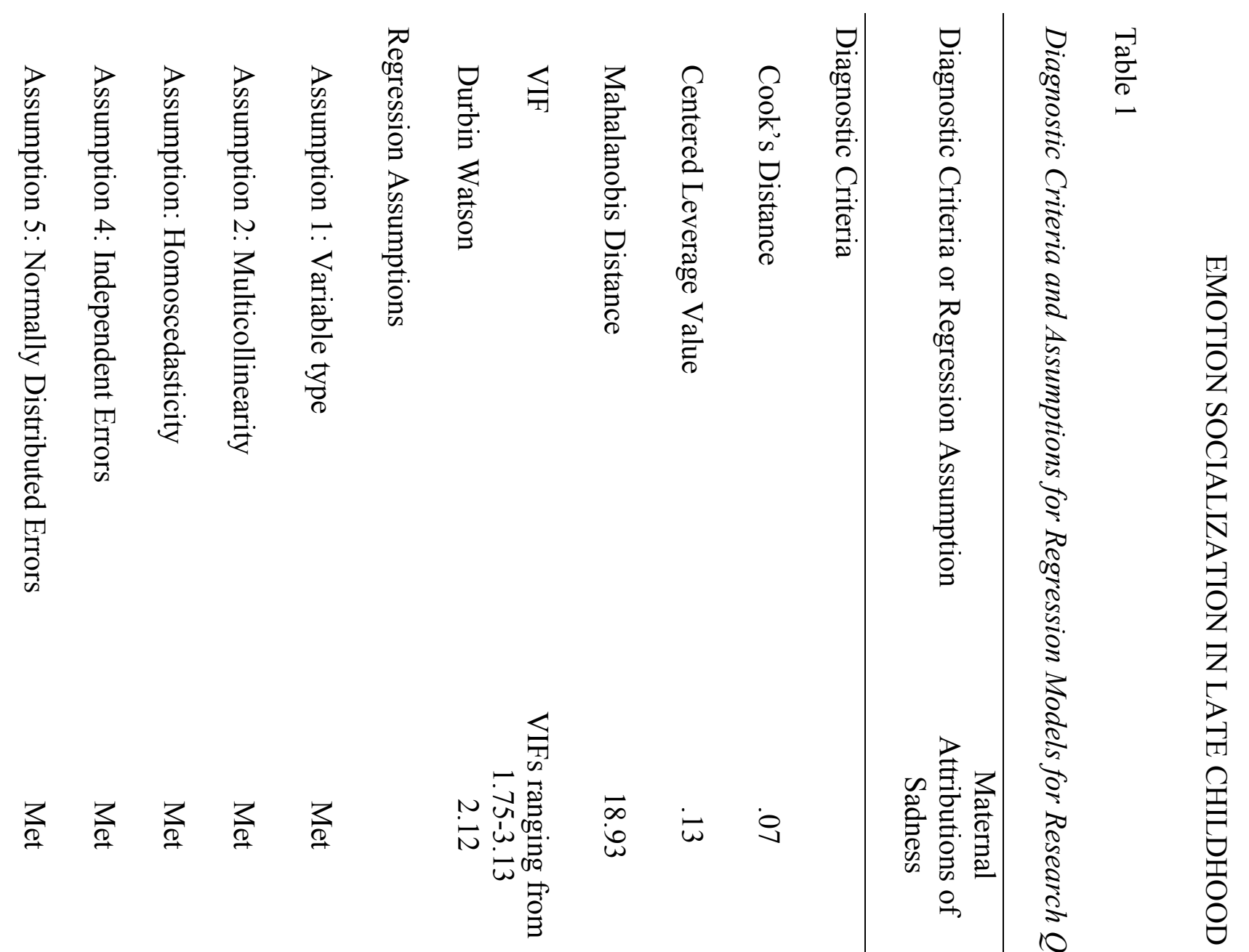

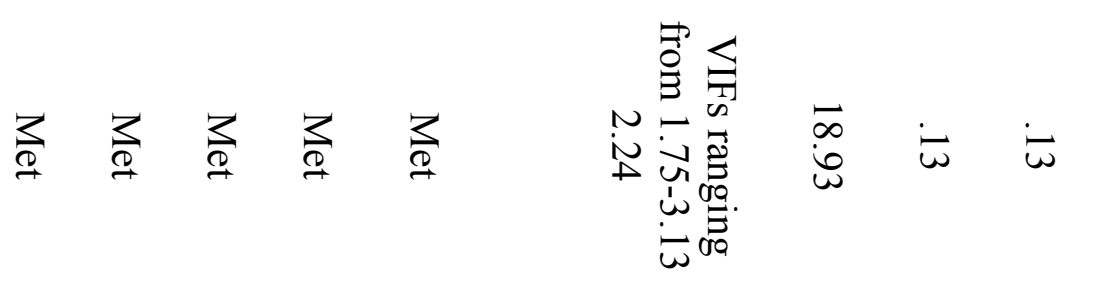

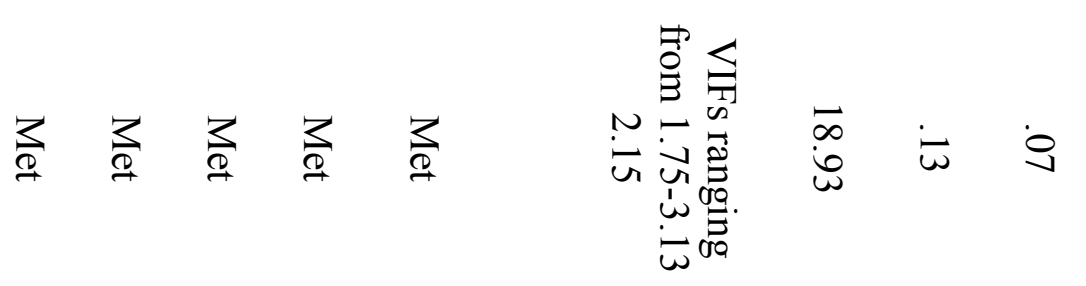

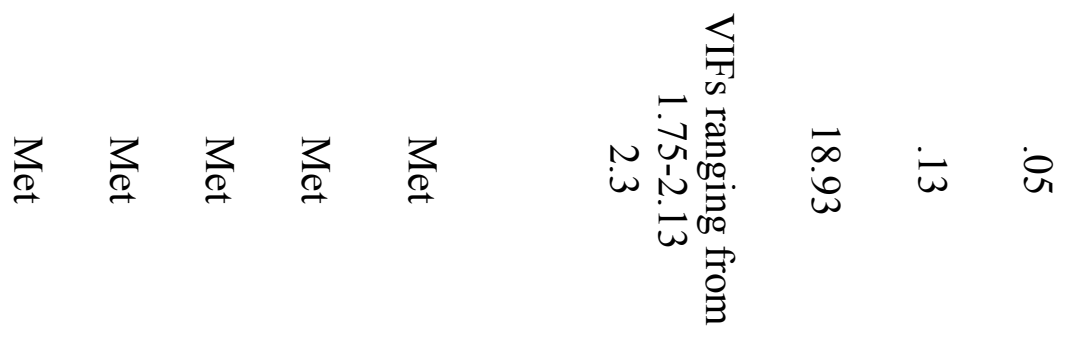

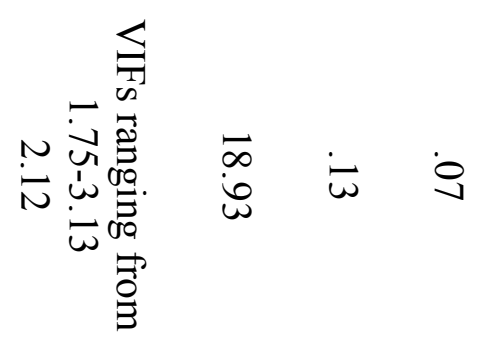

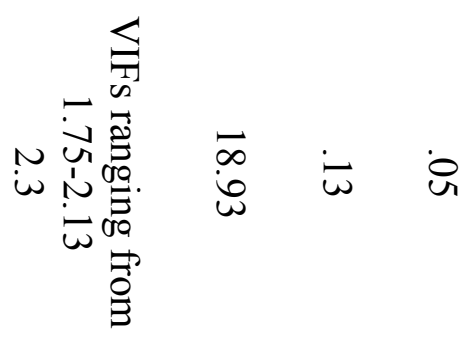




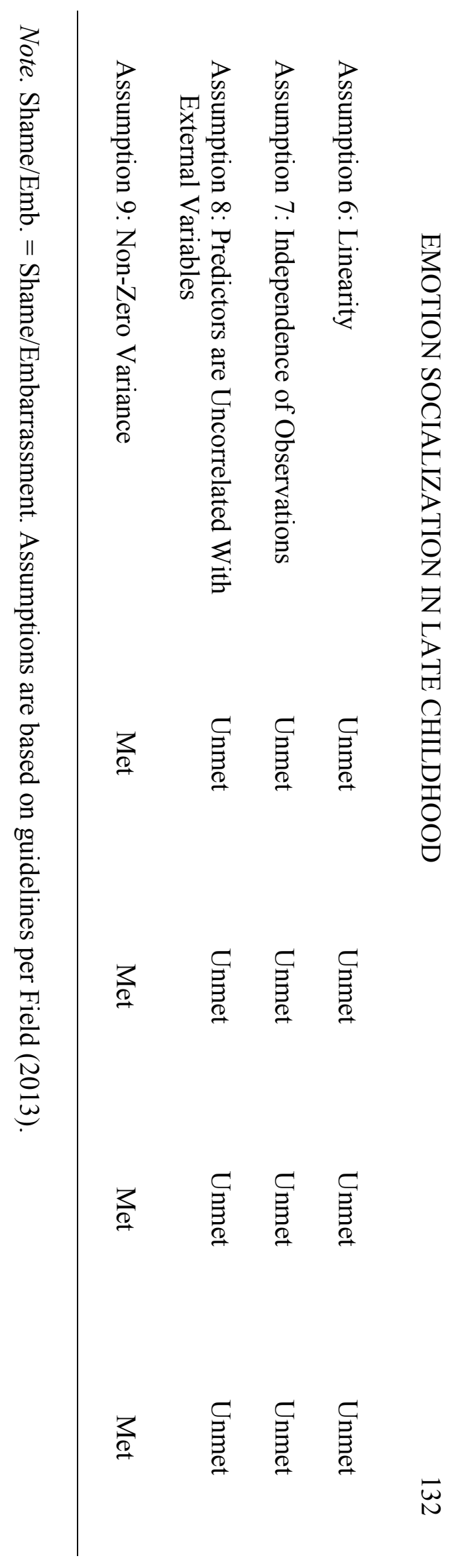



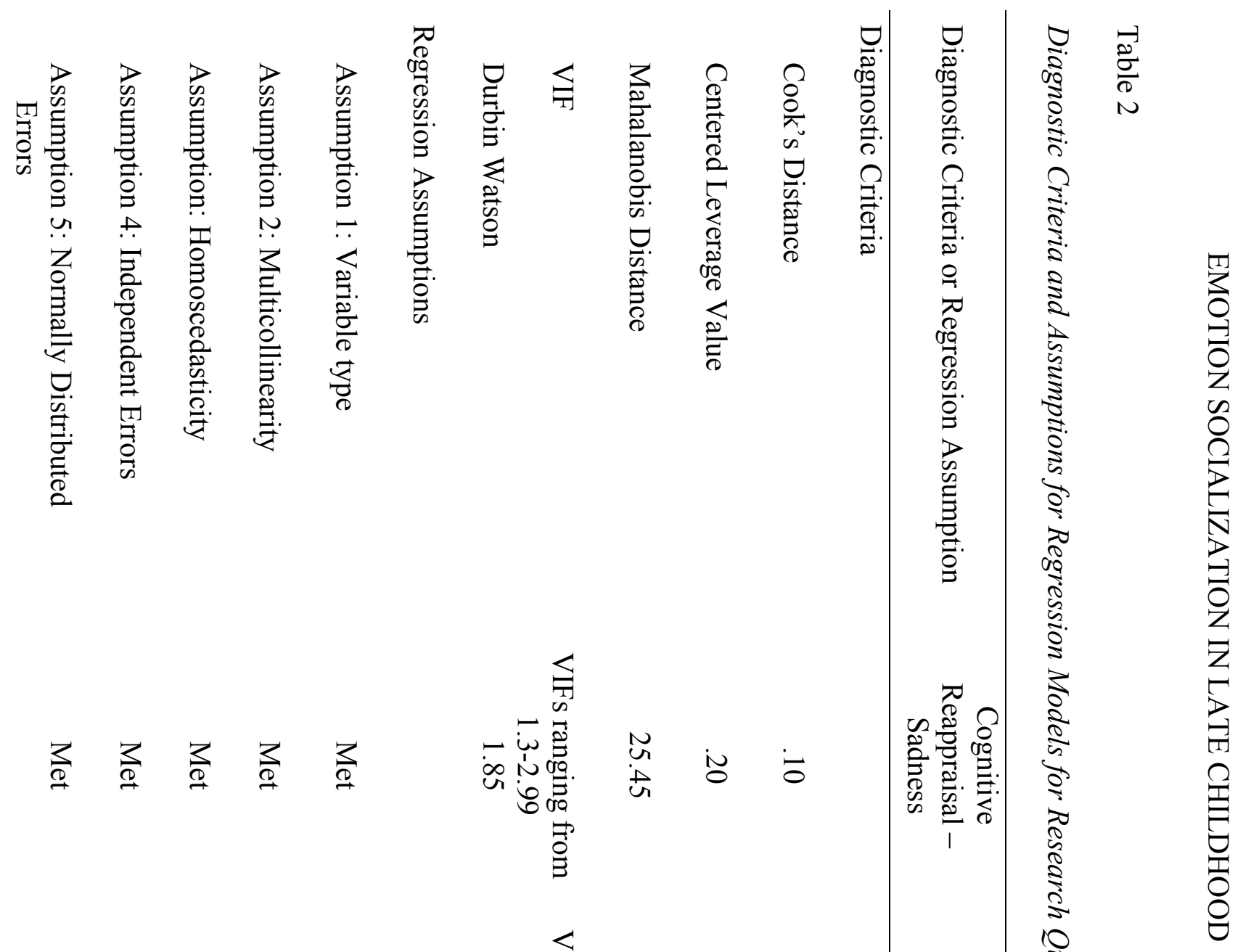

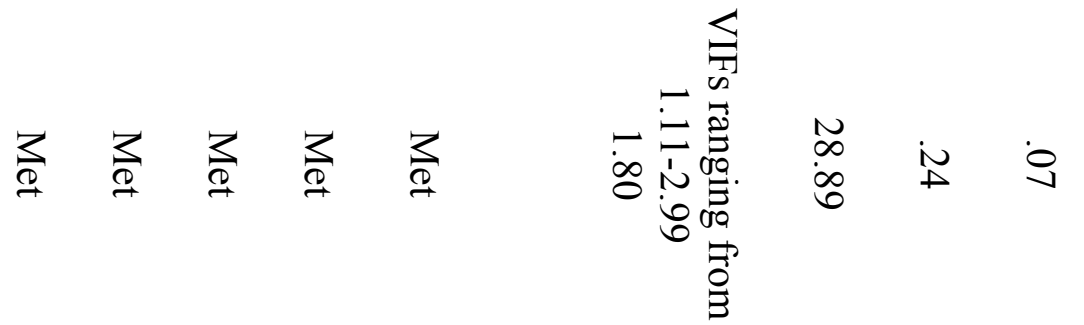

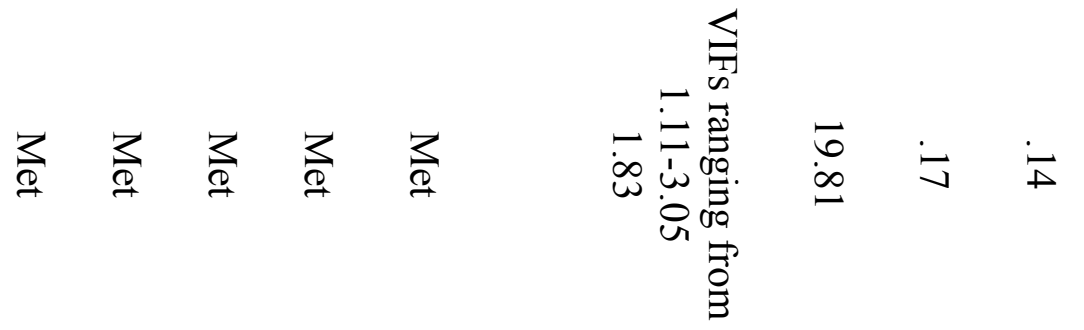

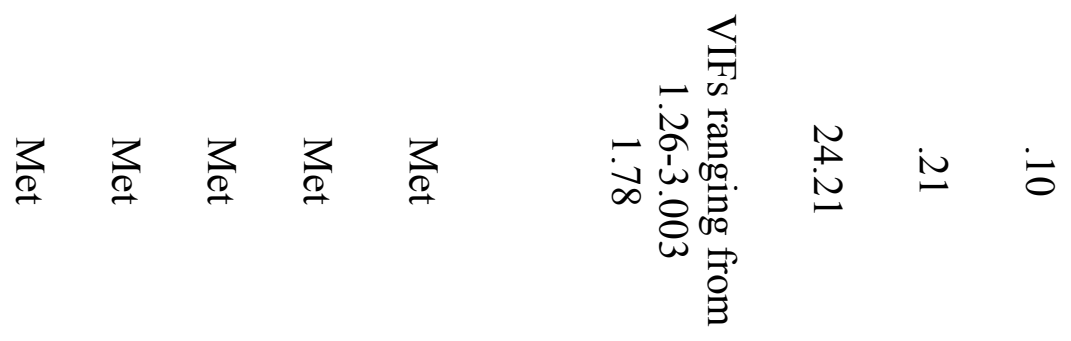

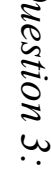

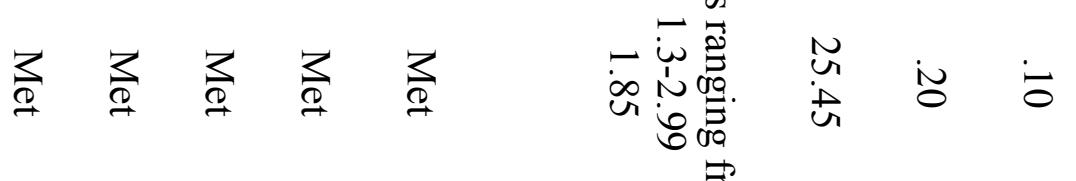




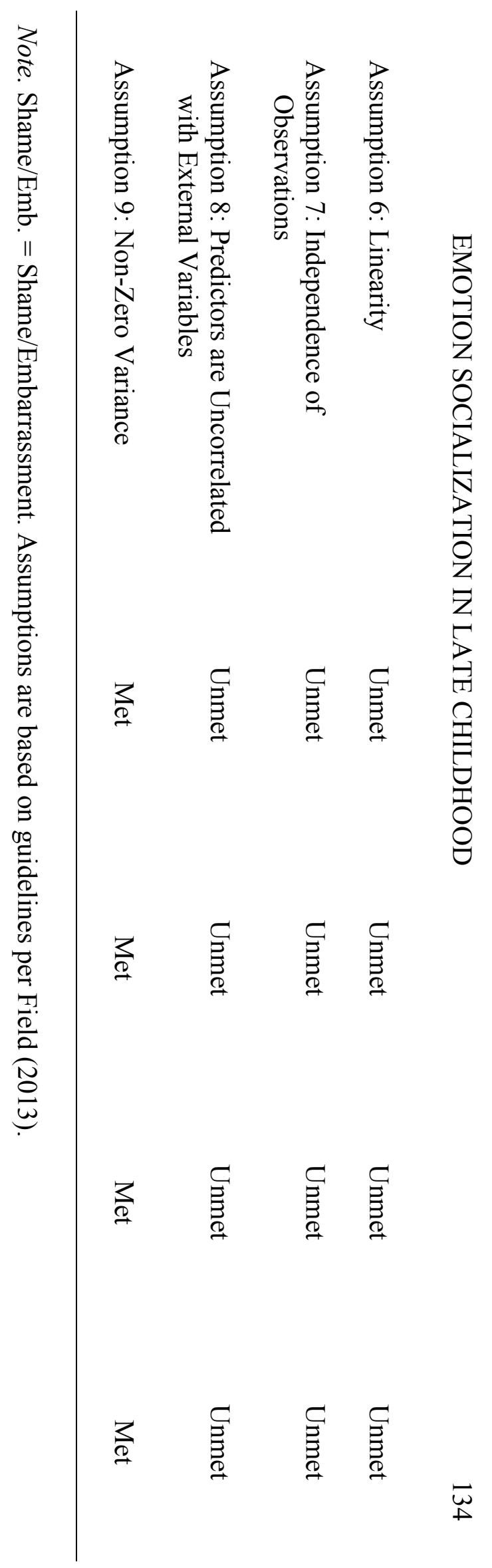




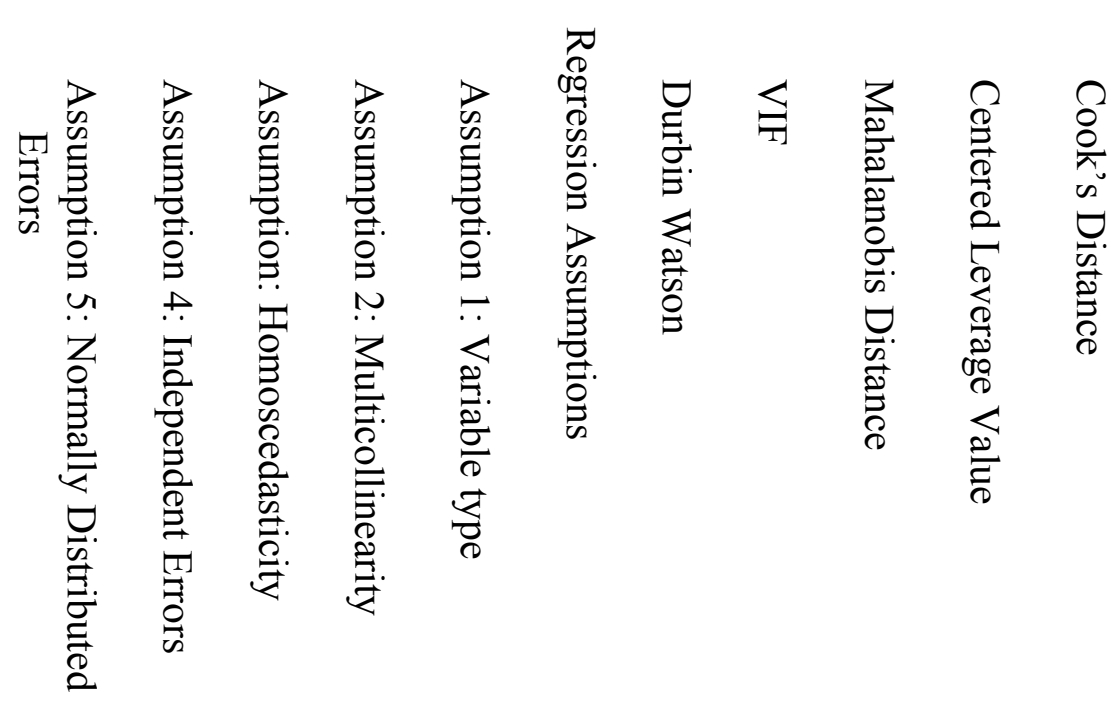

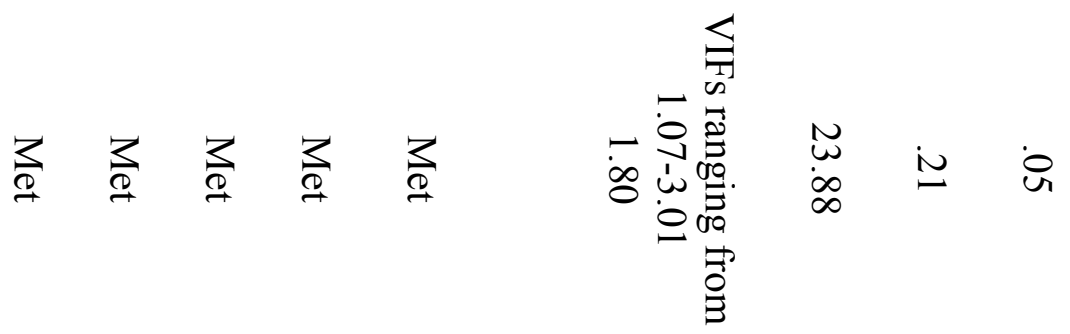

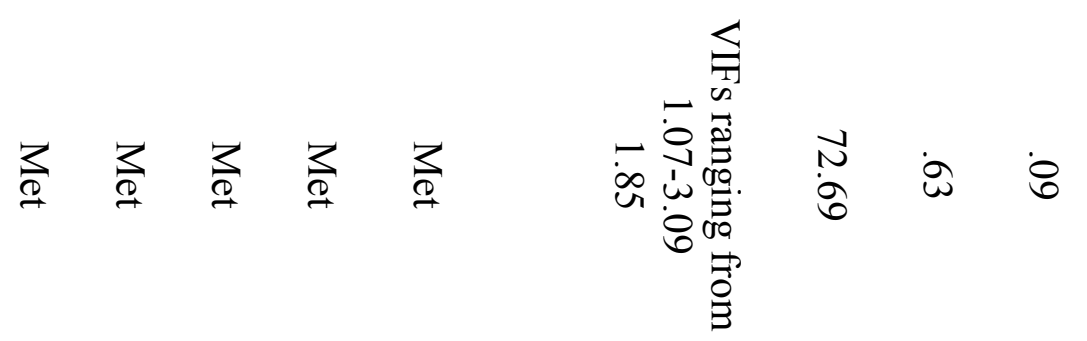

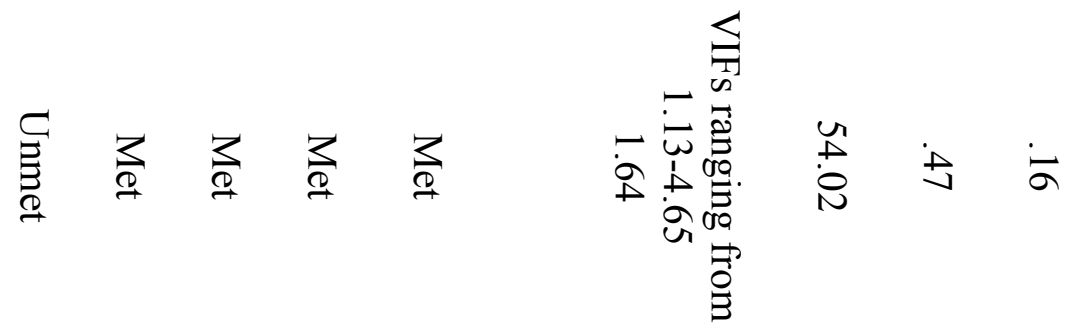

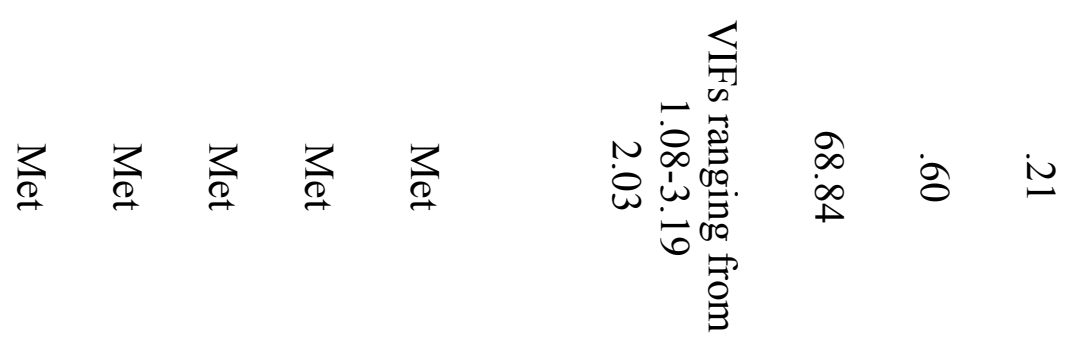

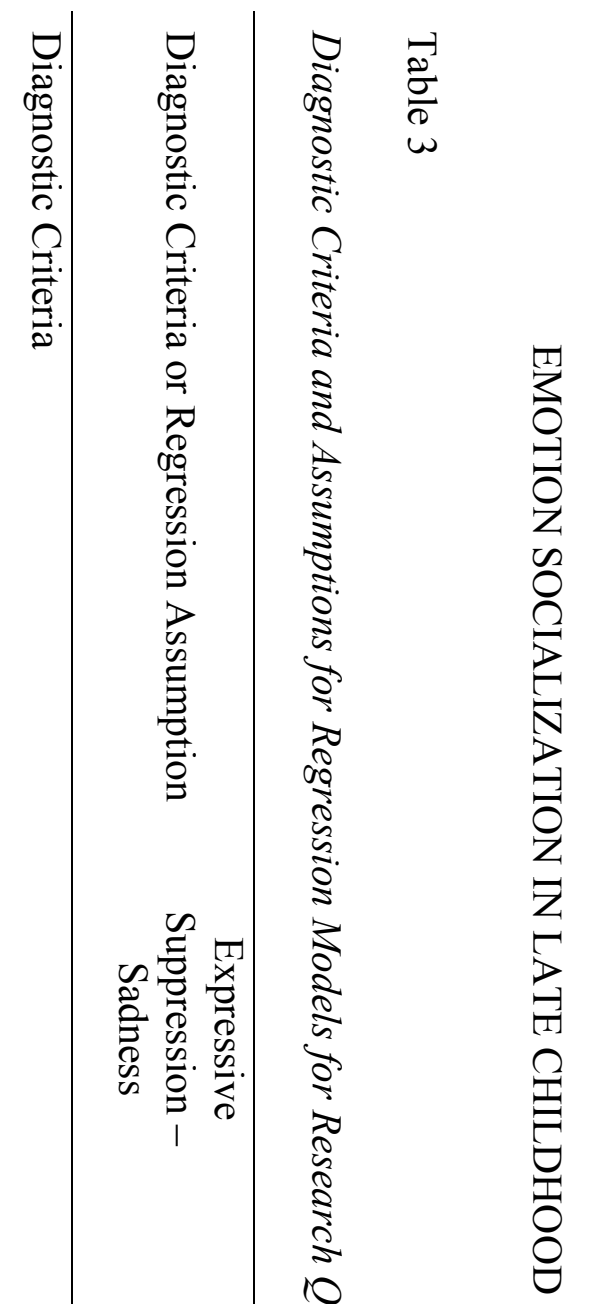




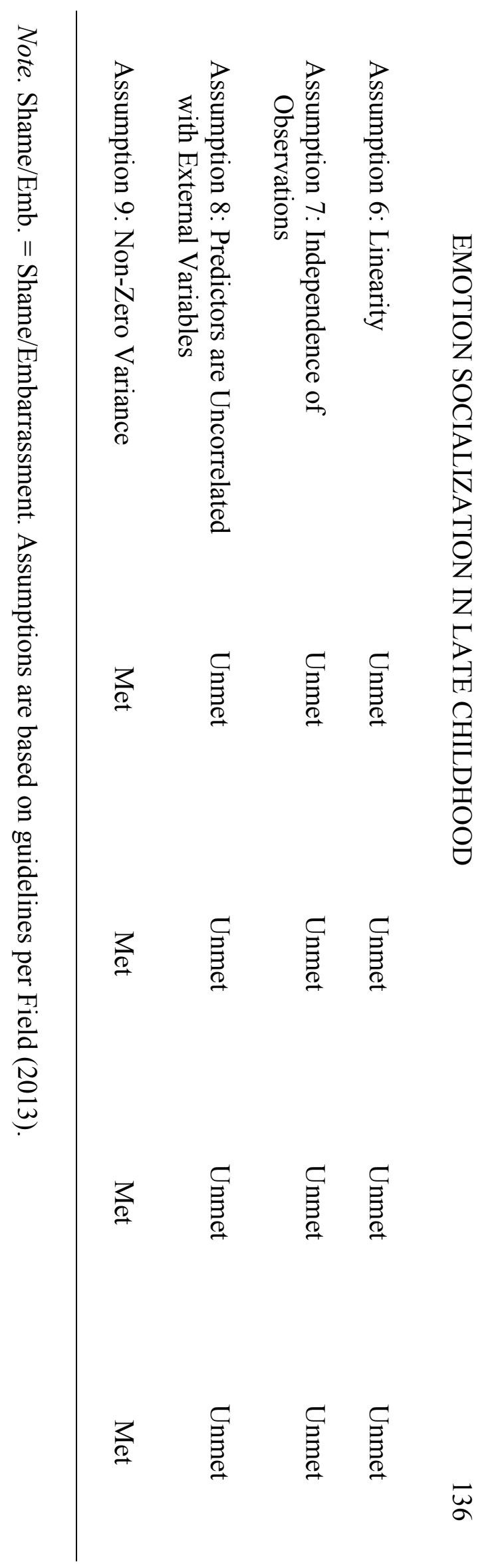



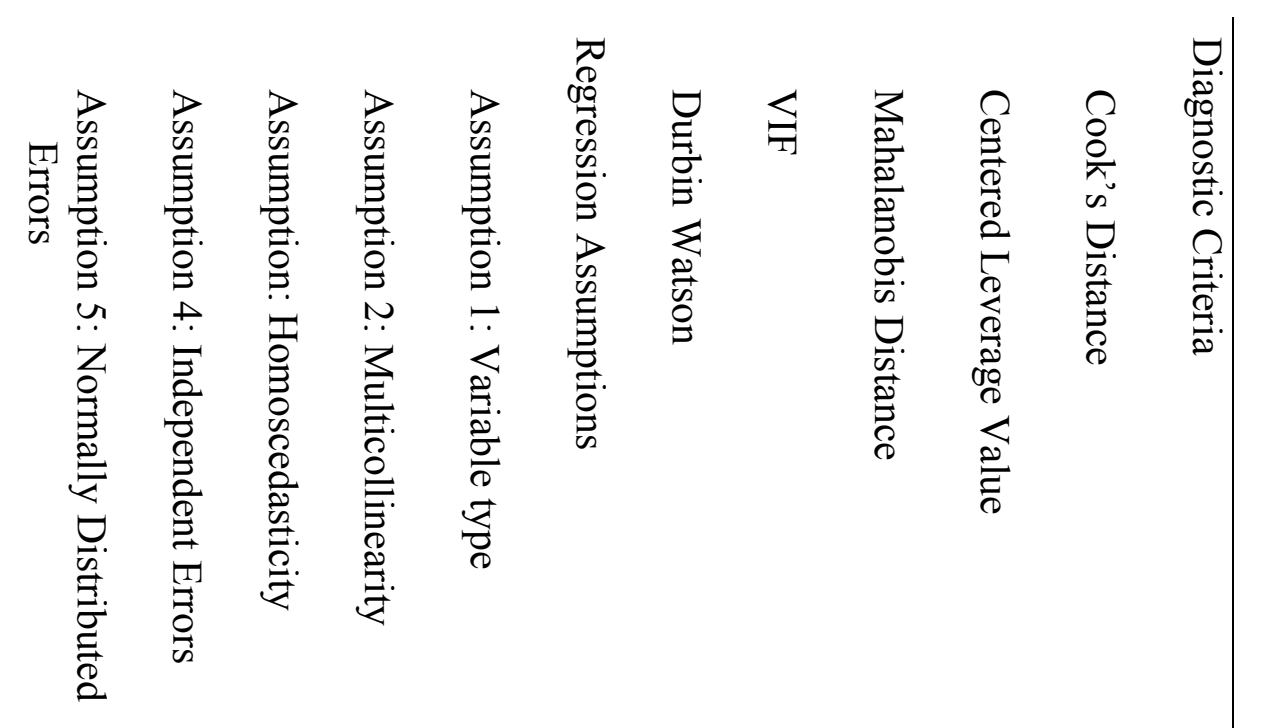

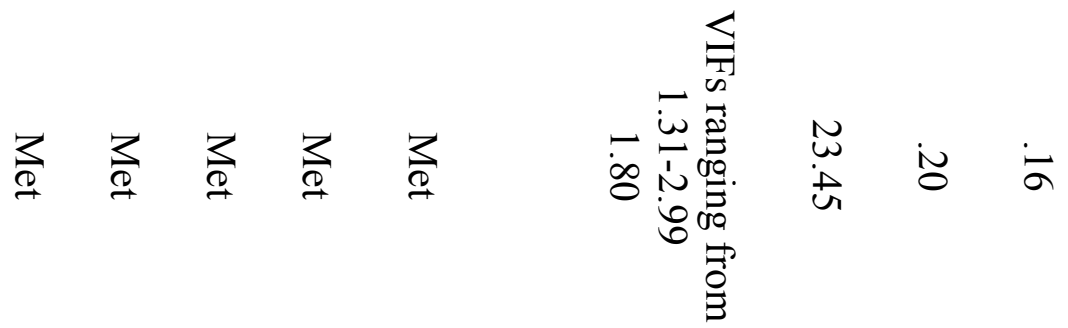

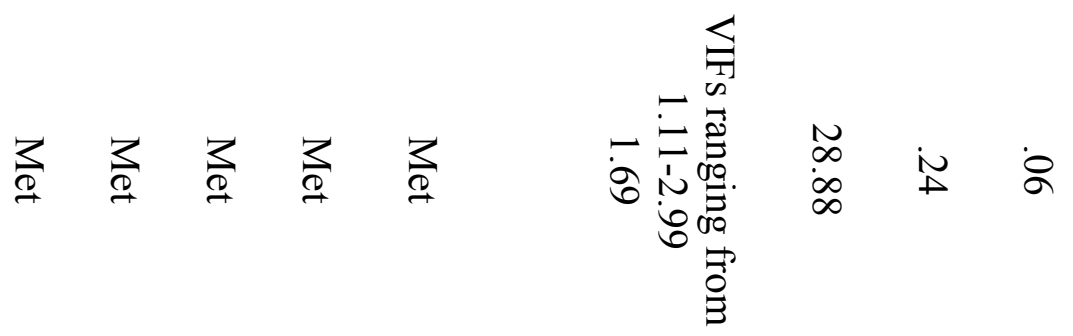

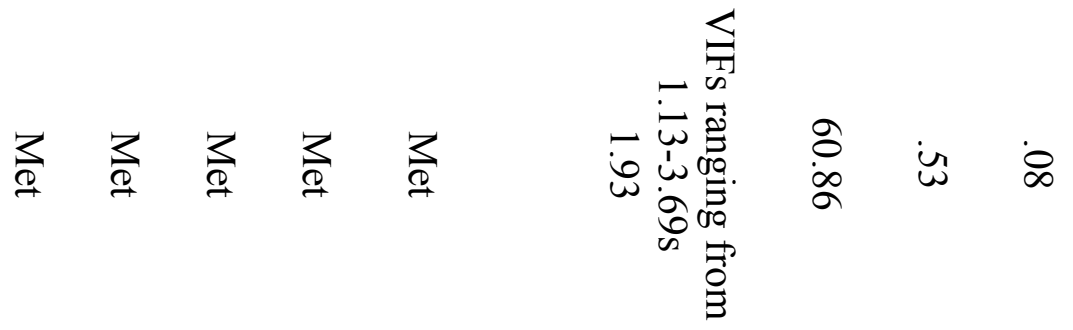

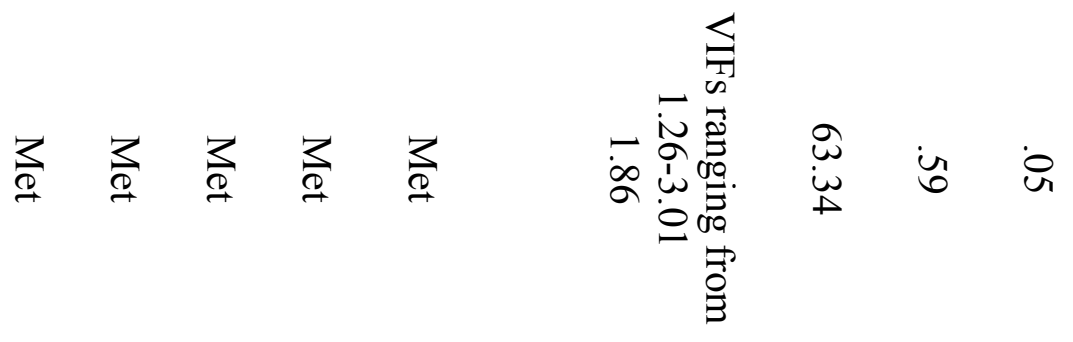

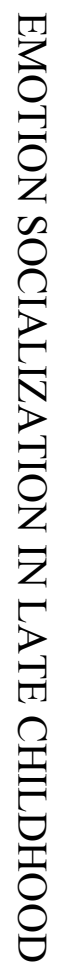

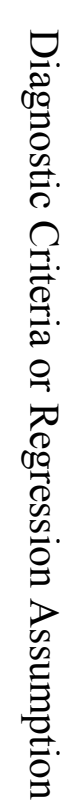

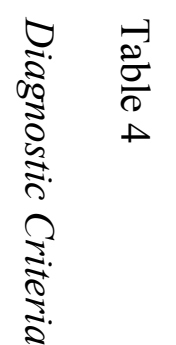

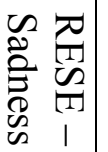

ฐ

$\frac{1}{\sum_{2}}$

(

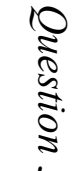

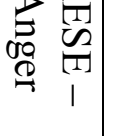

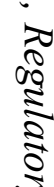

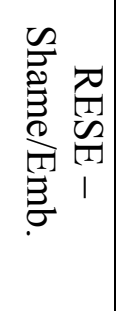




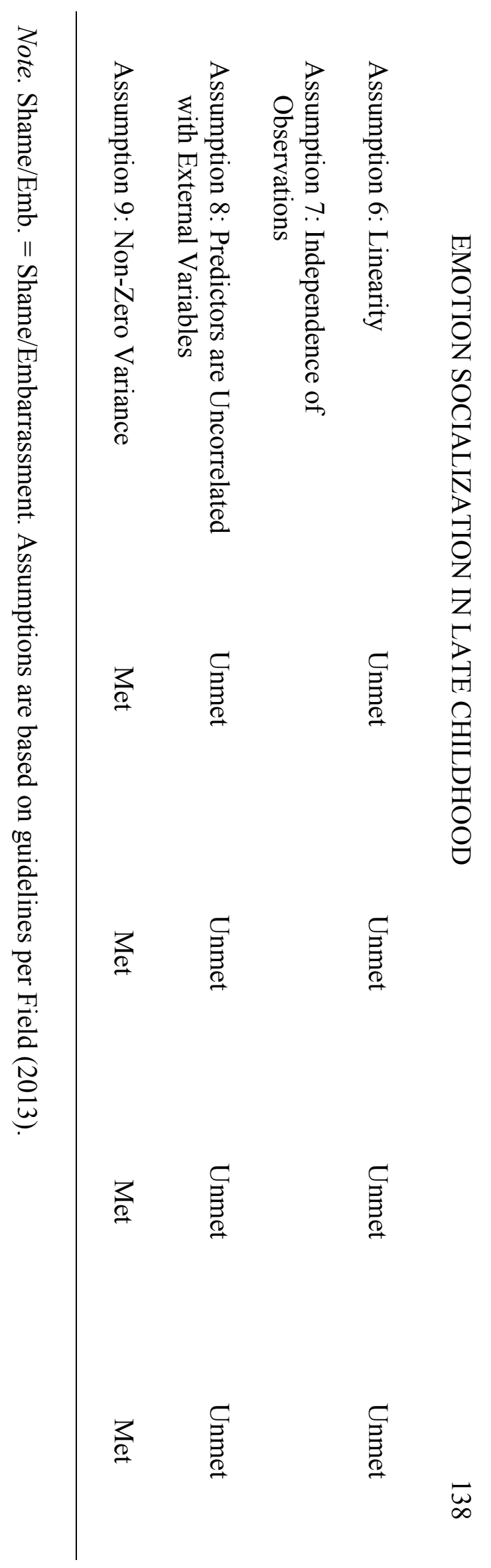


Table 5

Descriptive Statistics for Study Variables of Interest

Variable

Temperament Variables

Negative Emotionality

$\begin{array}{lllll}159 & 1.86 & .46 & 1.00 & 3.07\end{array}$

Soothability

159

$\begin{array}{llll}3.62 & .72 & 2.00 & 5.00\end{array}$

Attention Focusing

159

$\begin{array}{llll}3.35 & .69 & 1.86 & 4.57\end{array}$

Inhibitory Control

159

3.51

.56

1.63

4.88

Maternal Attributions Variables

Attributions of Sadness

159

3.40

.75

1.00

4.75

Attributions of Anger

159

$\begin{array}{llll}3.43 & .92 & 1.00 & 5.00\end{array}$

Attributions of Fear

159

$\begin{array}{llll}3.49 & .91 \quad 1.00 \quad 5.00\end{array}$

Attributions of

159

3.50

.87

1.00

5.00

Shame/Embarrassment

Emotion Socialization Variables

Supportive - Sadness

$\begin{array}{llll}6.17 & .76 & 3.25 & 7.00\end{array}$

Nonsupportive - Sadness

$\begin{array}{lllll}123 & 1.55 & .71 & 1.00 & 4.60\end{array}$

Nonsupportive - Anger

$\begin{array}{lllll}123 & 2.03 & .87 & 1.00 & 5.75\end{array}$

Supportive - Anger

$\begin{array}{lllll}123 & 5.69 & 1.05 & 1.50 & 7.00\end{array}$

Nonsupportive - Fear

$123 \quad 1.55$

.84

1.00

4.75

Supportive - Fear

123

$\begin{array}{llll}6.00 & .80 & 3.33 & 7.00\end{array}$

Nonsupportive - Shame/Embarrassment

$123 \quad 1.68$

$.86 \quad 1.00$

5.33

Supportive Shame/Embarrassment

123

$\begin{array}{lllll}123 & 5.91 & 1.13 & 2.00 & 7.00\end{array}$




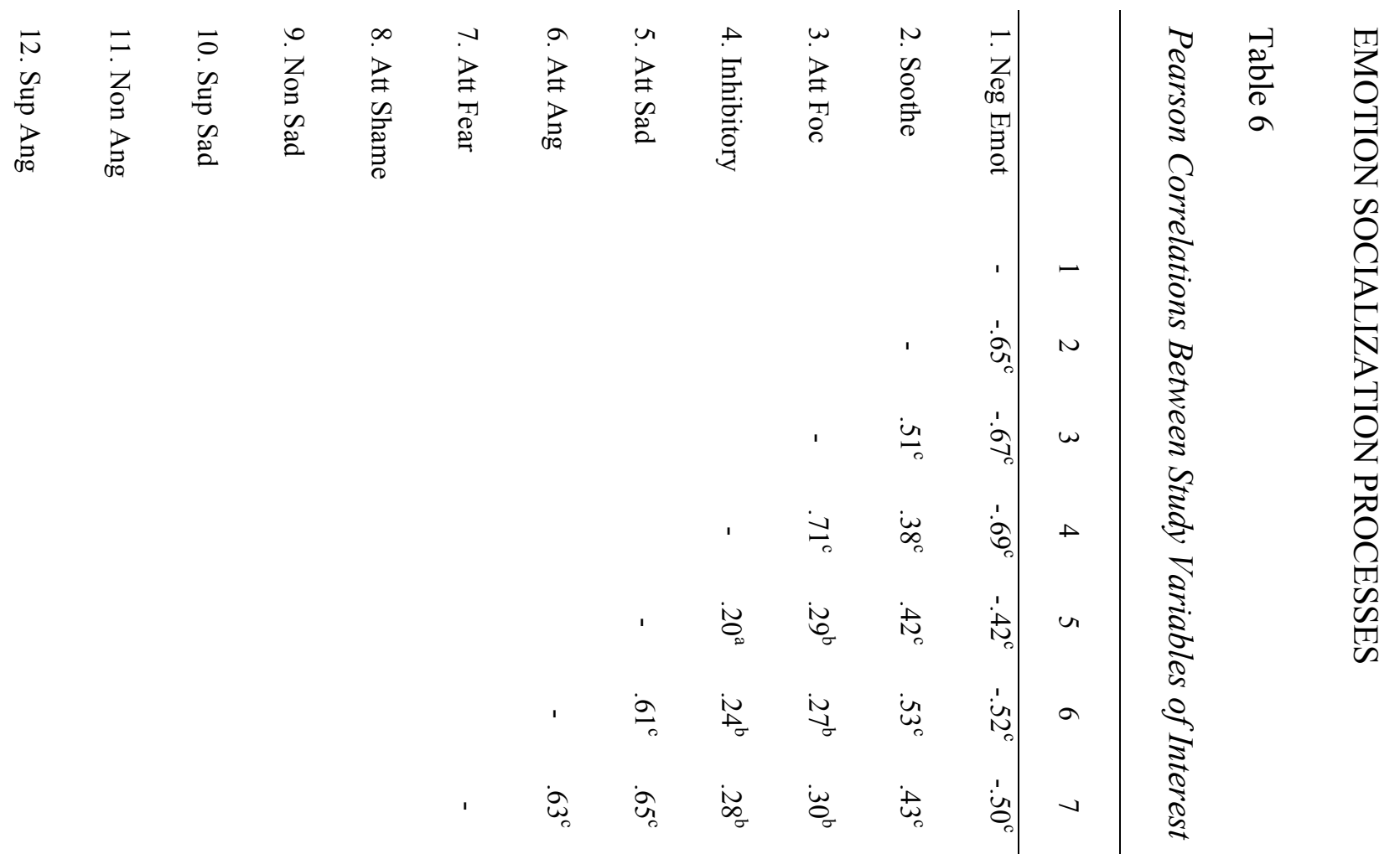

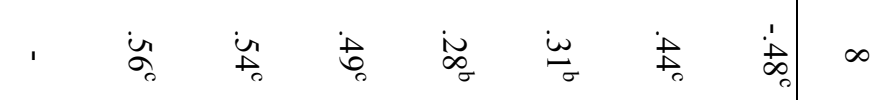

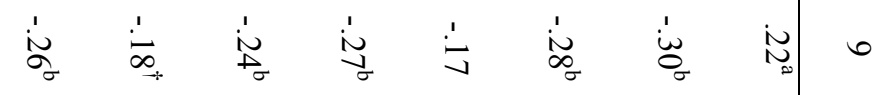

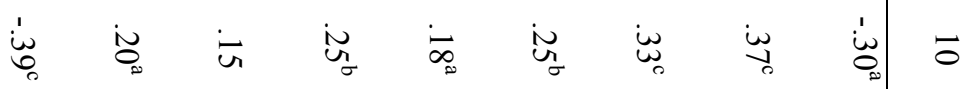

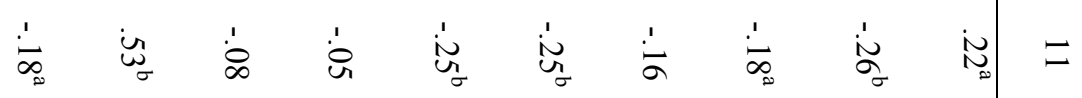

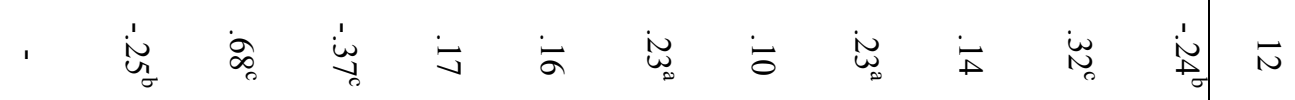

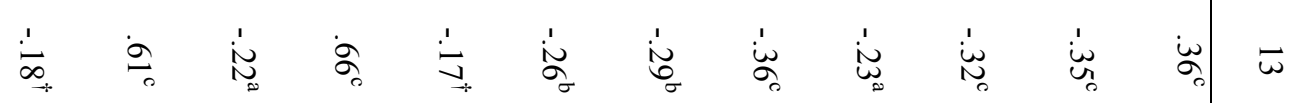

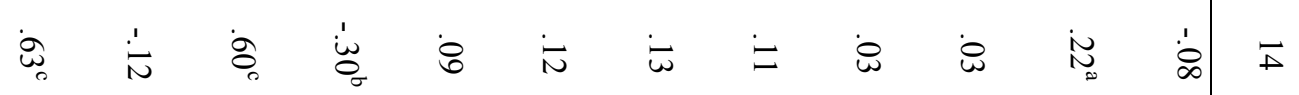

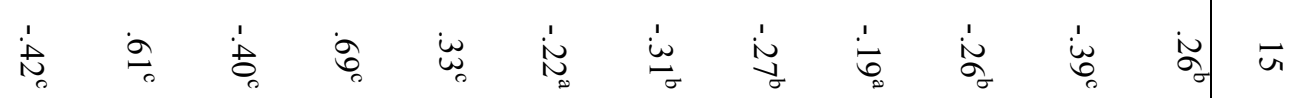

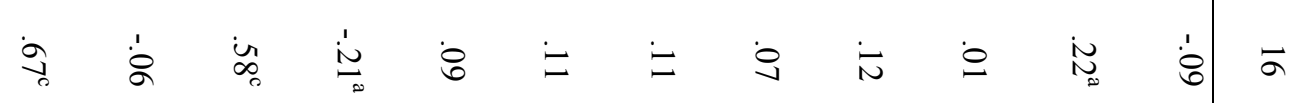

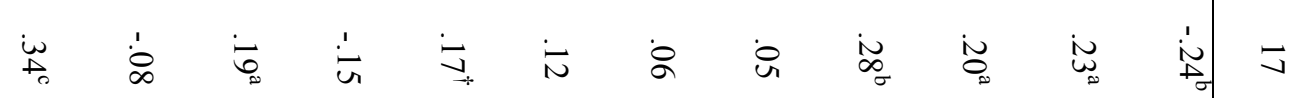

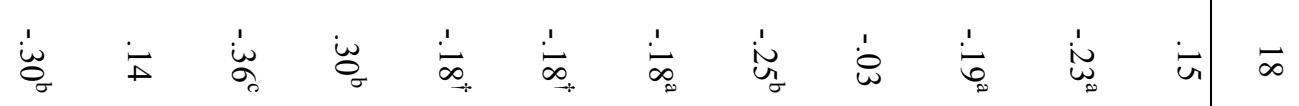

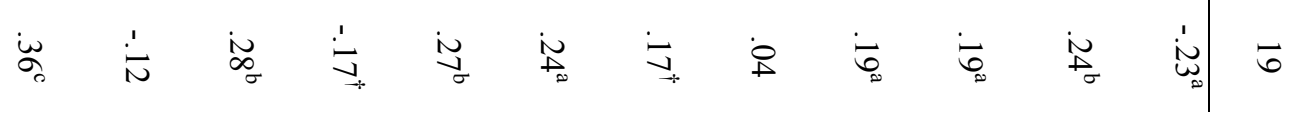




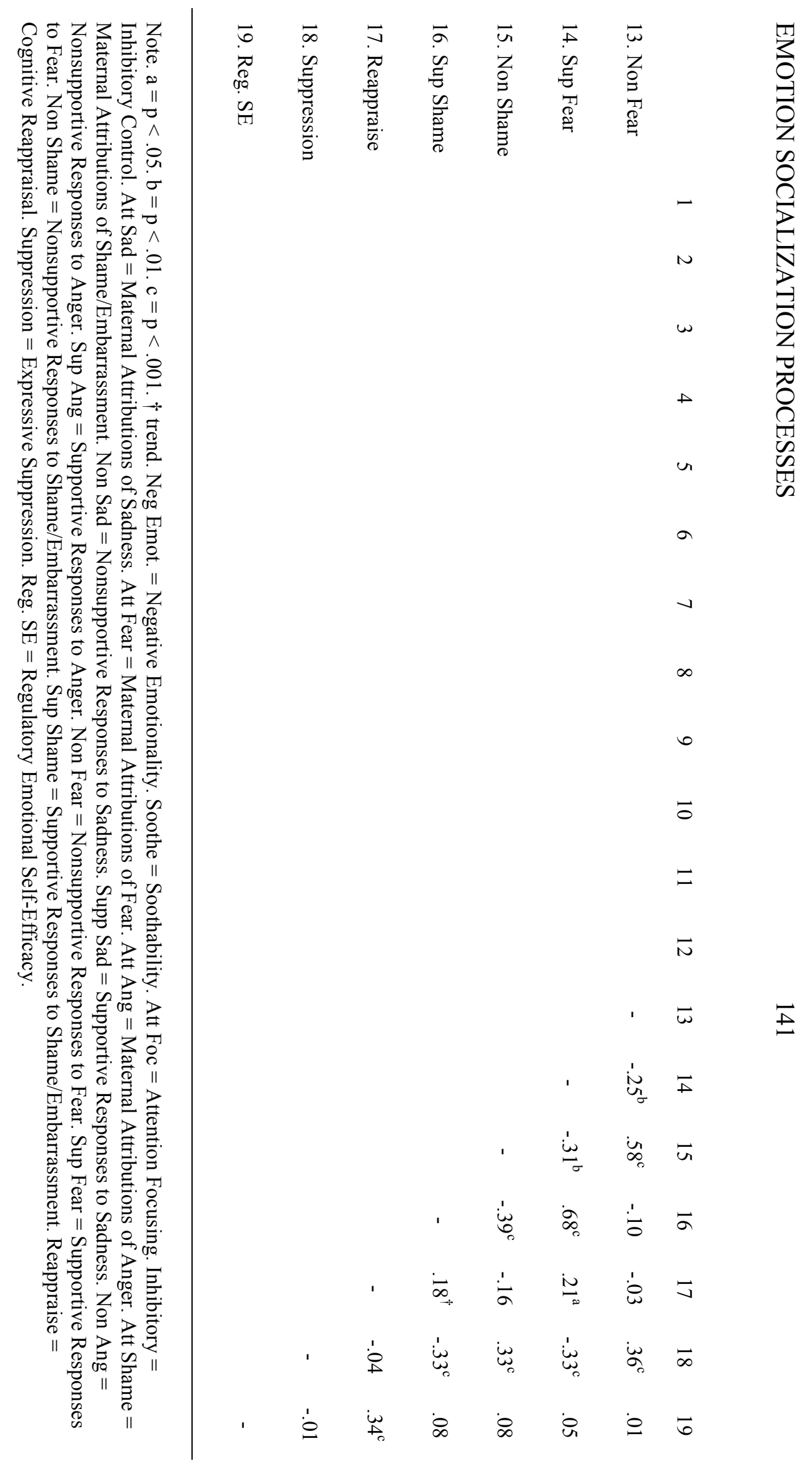




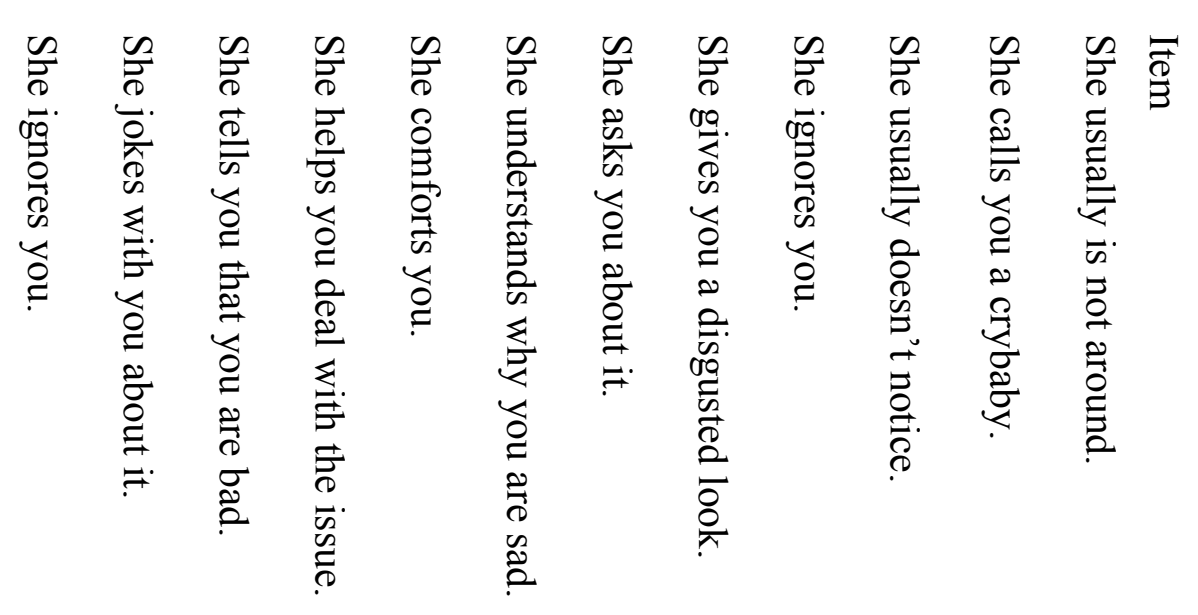

$$
\begin{aligned}
& \text { 崖崖总总 } \\
& \text { 变要商 }
\end{aligned}
$$

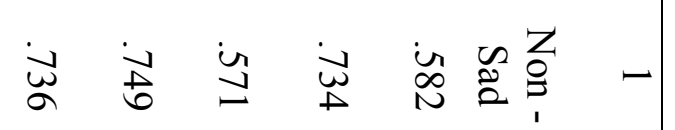

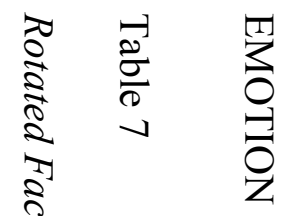

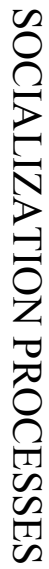$$
\text { 颉 N }
$$$$
\text { के }
$$

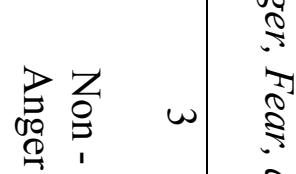

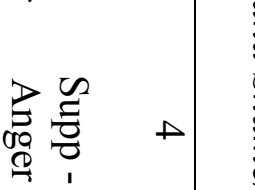

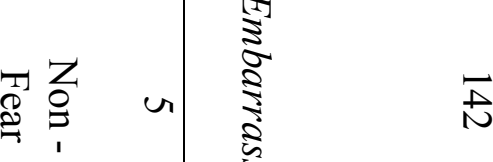




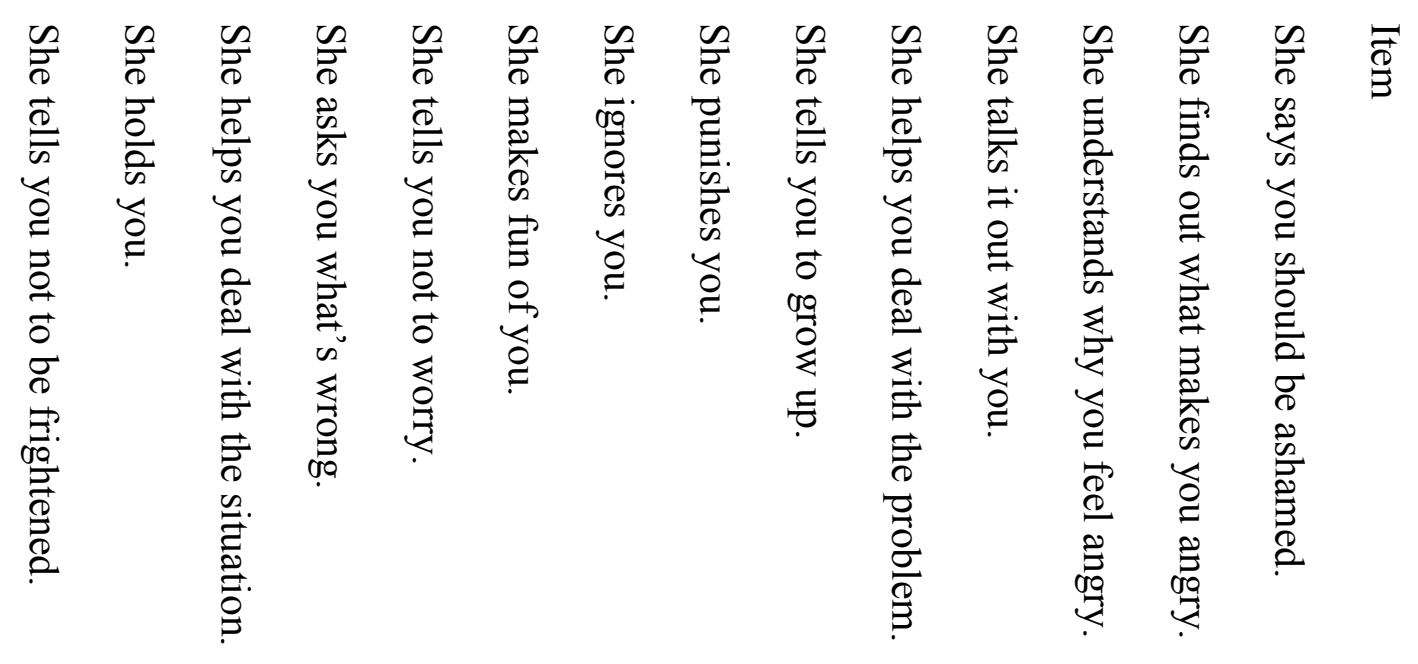

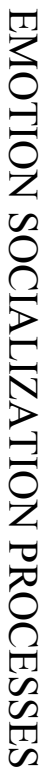

$$
\begin{aligned}
& \text { ڤొڤ } \\
& \text { ڤैڤ }
\end{aligned}
$$

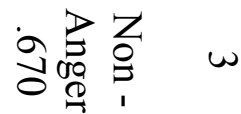

$$
\begin{aligned}
& \text { ư ư } \\
& \text { نூ } \\
& \begin{array}{l}
T \\
0
\end{array}
\end{aligned}
$$

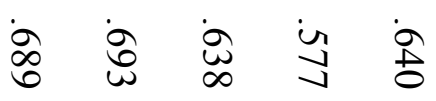

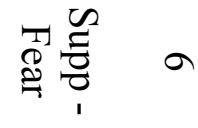

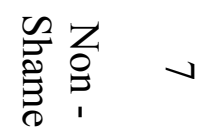

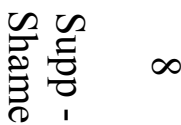




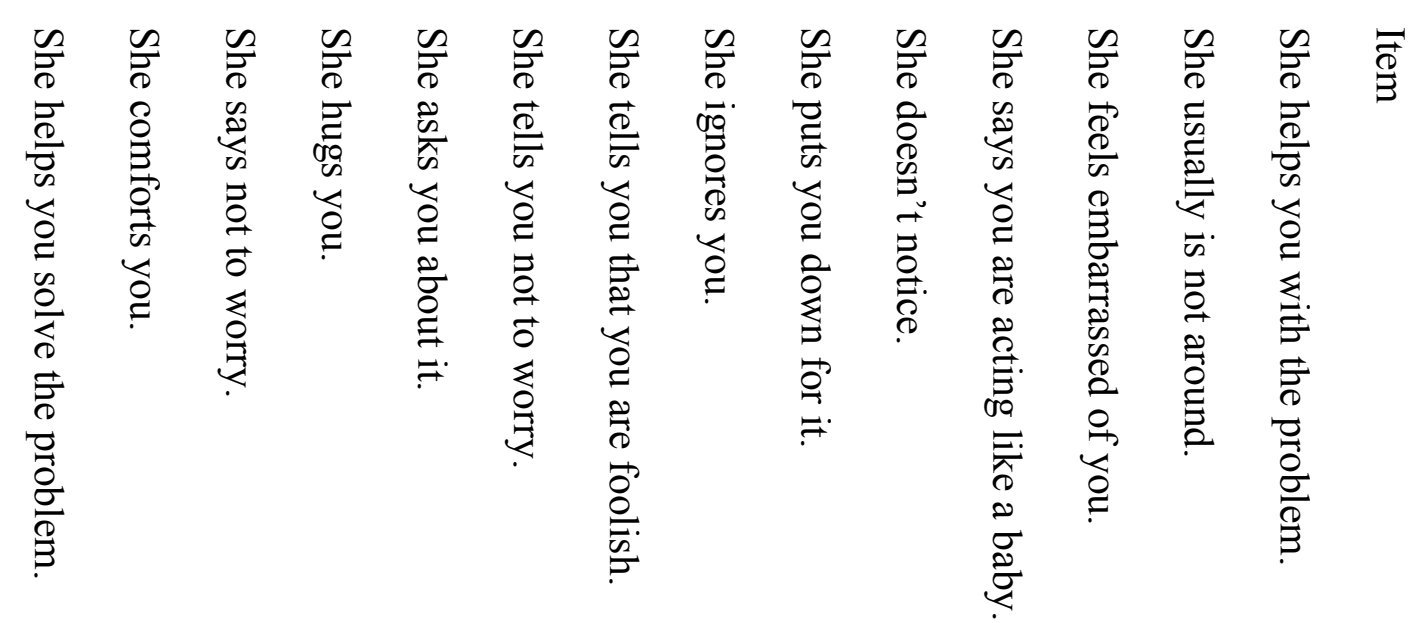

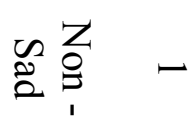

$$
\begin{aligned}
& \text { N }
\end{aligned}
$$

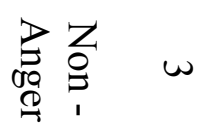

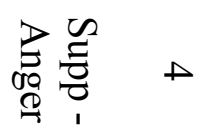

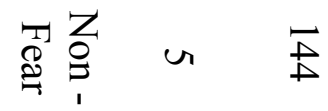

$$
\begin{aligned}
& \text { ज }
\end{aligned}
$$

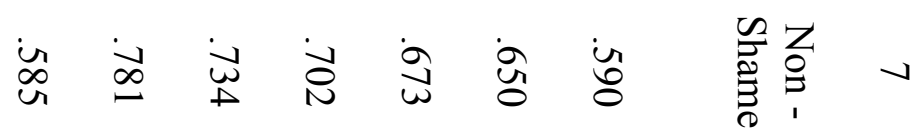

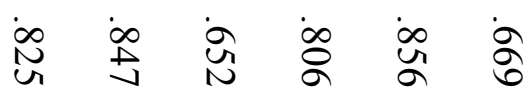

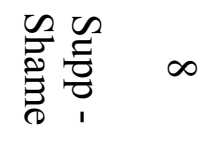




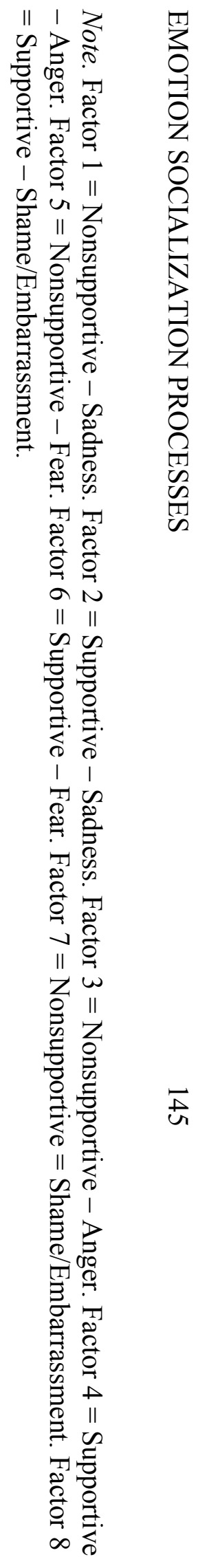




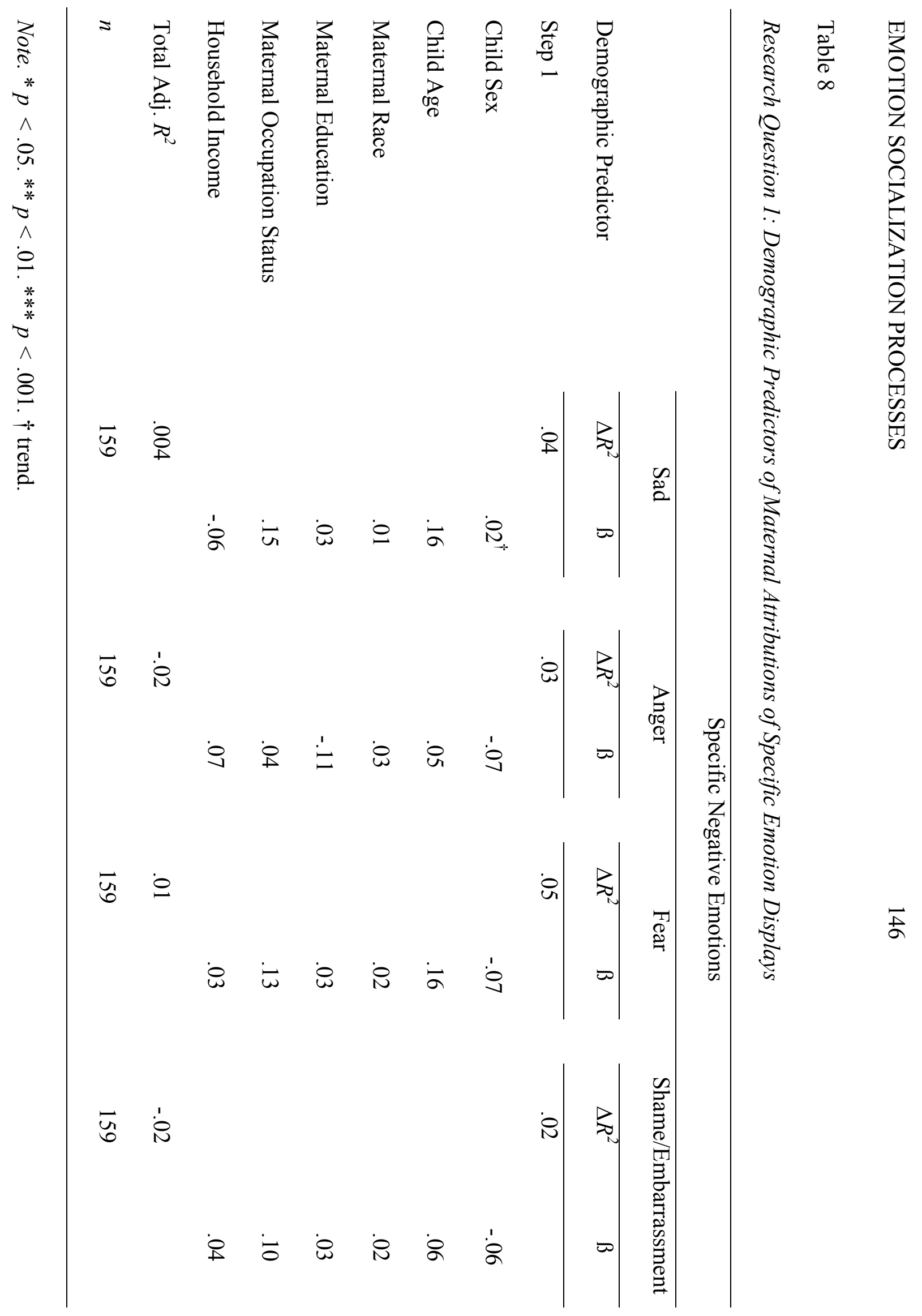




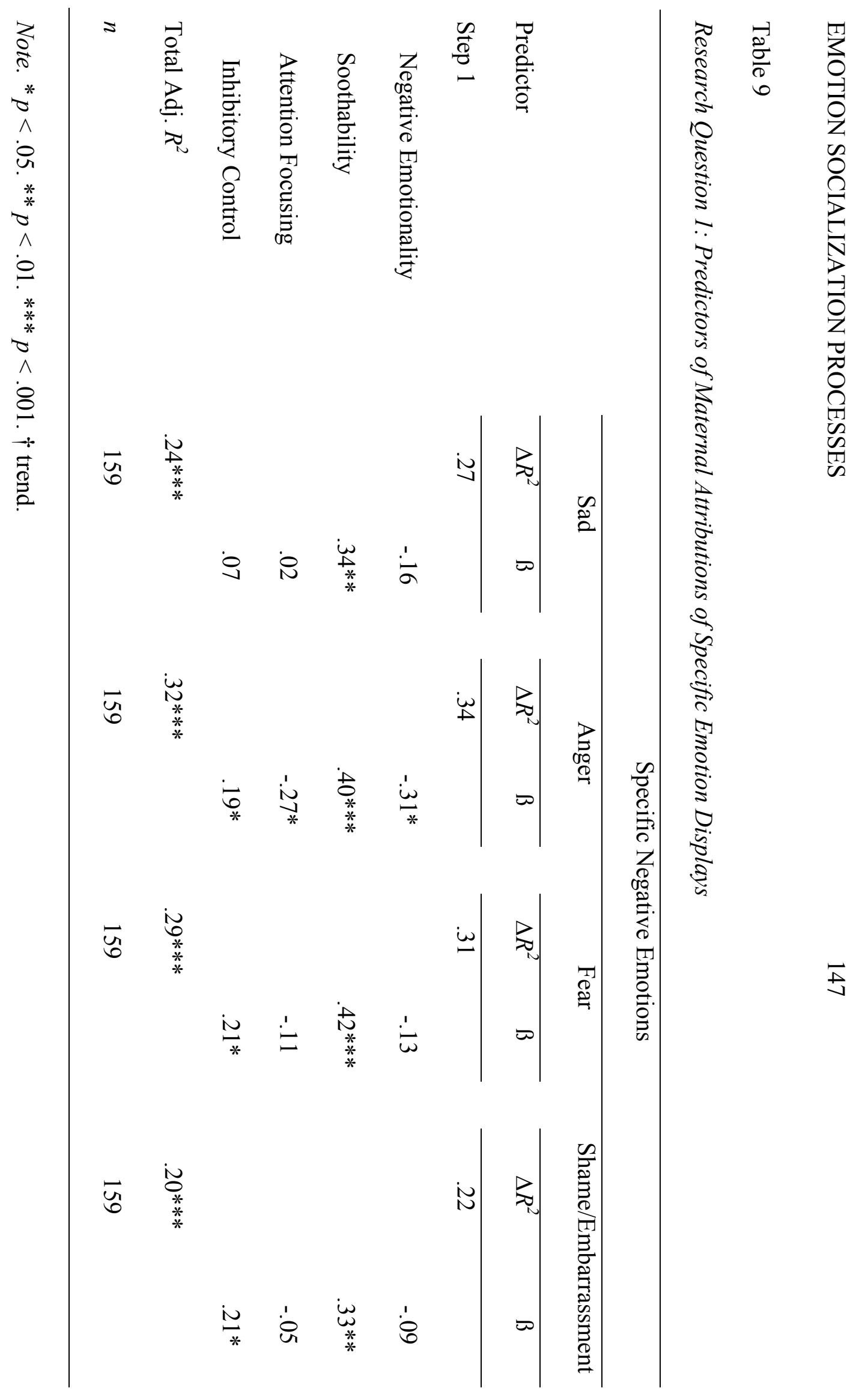




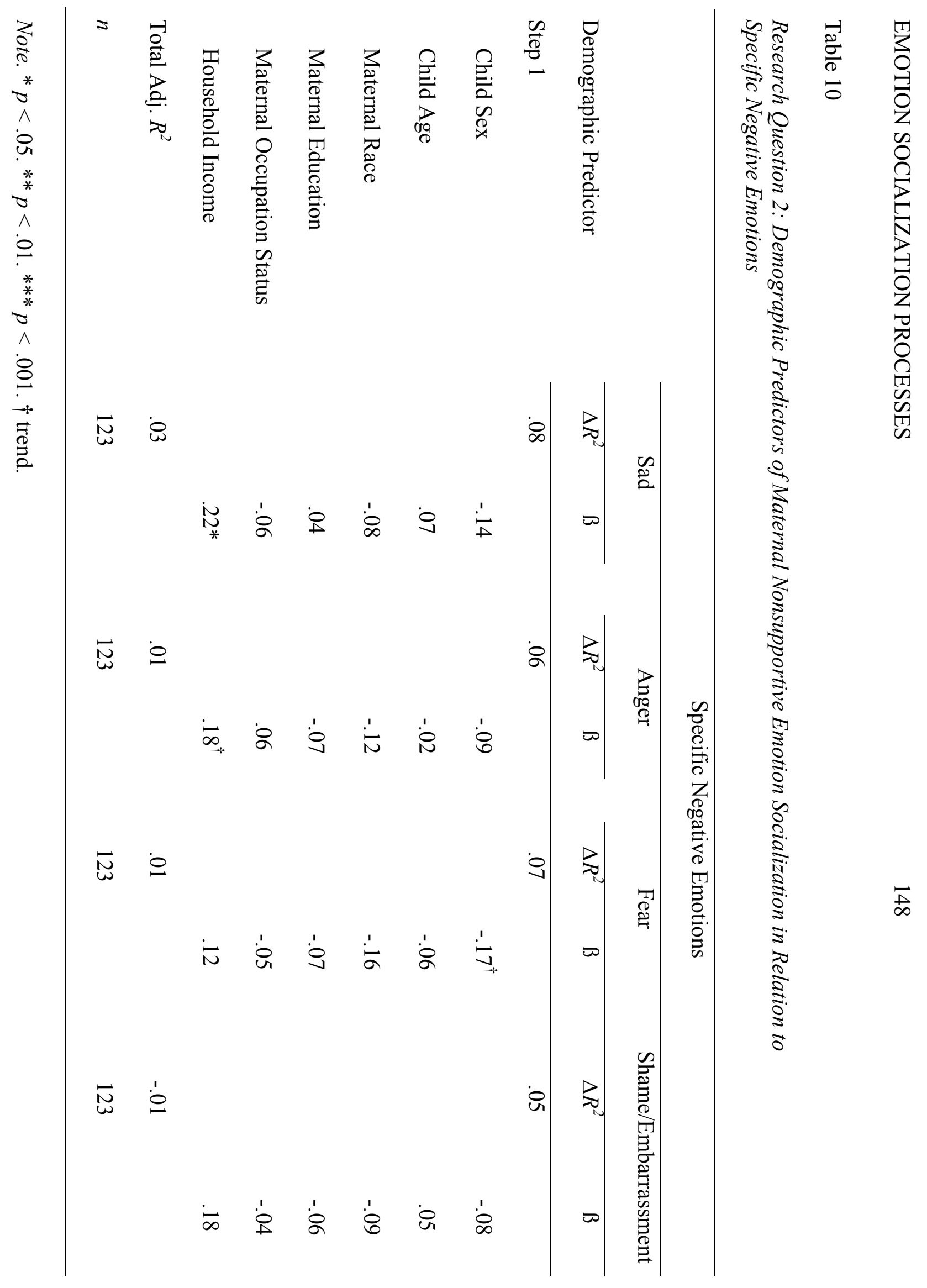




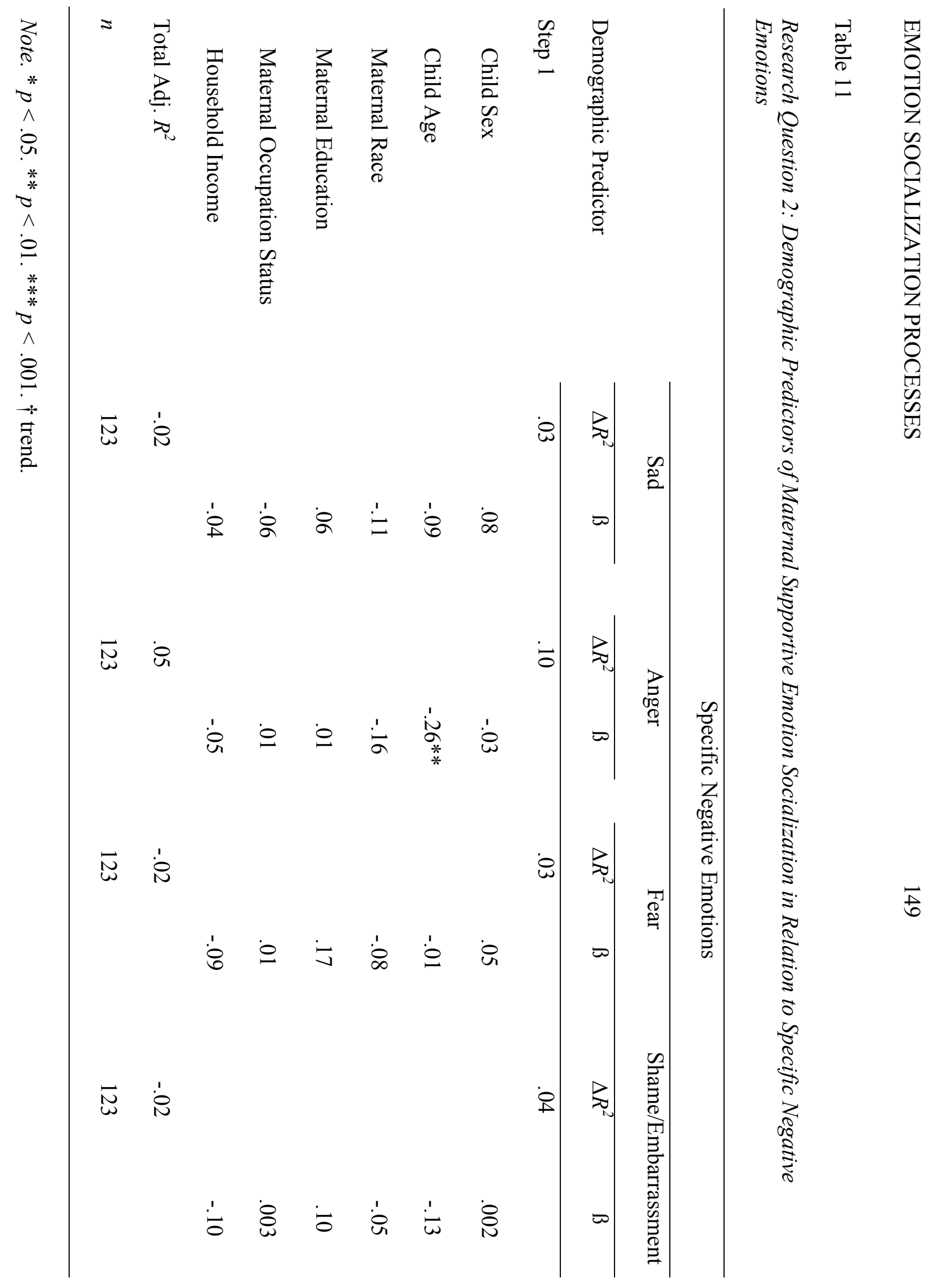


Table 12

Research Question 2: Indirect Effects of Maternal Attributions of Specific Negative Emotion Displays on Relation Between Child Temperament and Maternal Emotion Socialization

\begin{tabular}{ccc}
\hline & Bootstrap & Bootstrap \\
Indirect Effect & $\begin{array}{c}\text { Estimate } \\
\text { (SE) }\end{array}$ & [LLCI, ULCI] \\
\hline
\end{tabular}

Sad - Nonsupportive Emotion Socialization

Negative emotionality $>$ Attributions of sadness $>$

Nonsupportive

Soothability $>$ Attributions of Sadness $>$ Nonsupportive

Attention focusing $>$ Attributions of Sadness $>$

Nonsupportive

Inhibitory control $>$ Attributions of Sadness $>$

Nonsupportive

Sad - Supportive Emotion Socialization

Negative emotionality $>$ Attributions of Sadness $>$

Supportive

Soothability $>$ Attributions of Sadness $>$ Supportive

Attention focusing $>$ Attributions of Sadness $>$ Supportive

Inhibitory control $>$ Attributions of Sadness $>$ Supportive

Anger - Nonsupportive Emotion Socialization

Negative emotionality $>$ Attributions of Anger $>$

Nonsupportive

Soothability $>$ Attributions of Anger $>$ Nonsupportive

Attention focusing $>$ Attributions of Anger $>$

Nonsupportive

Inhibitory control > Attributions of Anger >

Nonsupportive
$.21(.10) \quad[.057, .429]^{*}$

$-.07(.06) \quad[-.206,-.003]^{*}$

$-.04(.03) \quad[-.124,-.004]^{*}$

$-.06(.04) \quad[-.175,-.004]^{*}$

$-.11(.09) \quad[-.322, .056]$

$.02(05) \quad[-.045, .142]$

$.02(.03) \quad[-.016, .091]$

$.04(.04) \quad[-.006, .152]$

$.28(.15) \quad[.046, .629]^{*}$

$-.10(.08) \quad[-.310, .023]$

$-.05(.04) \quad[-.153,-.005]^{*}$

$-.08(.06) \quad[-.233,-.009]^{*}$ 
Indirect Effect

Anger - Supportive Emotion Socialization

Negative emotionality $>$ Attributions of Anger $>$

Supportive

Soothability $>$ Attributions of Anger $>$ Supportive

Attention focusing $>$ Attributions of Anger $>$ Supportive

Inhibitory control $>$ Attributions of Anger $>$ Supportive

Fear - Nonsupportive Emotion Socialization

Negative emotionality $>$ Attributions of Fear

Nonsupportive

Soothability $>$ Attributions of Fear $>$ Nonsupportive

Attention focusing $>$ Attributions of Fear $>$ Nonsupportive

Inhibitory control $>$ Attributions of Fear $>$ Nonsupportive

Fear - Supportive Emotion Socialization

Negative emotionality $>$ Attributions of Fear $>$ Supportive

Soothability $>$ Attributions of Fear $>$ Supportive

Attention focusing $>$ Attributions of Fear $>$ Supportive

Inhibitory control $>$ Attributions of Fear $>$ Supportive

Shame/Embarrassment - Nonsupportive Emotion

Socialization

Negative emotionality $>$ Attributions of Shame/Emb. >

Nonsupportive

Soothability $>$ Attributions of Shame/Emb. $>$

Nonsupportive

Attention focusing > Attributions of Shame/Emb. >

Nonsupportive

Inhibitory control $>$ Attributions of Shame/Emb. >

Nonsupportive
Bootstrap Bootstrap
Estimate [LLCI, ULCI]
(SE)

$-.33(.16) \quad[-.674,-.060]^{*}$

$.08(.11) \quad[-.121, .314]$

$.06(.04) \quad[.003, .167]^{*}$

$.08(.06) \quad[-.005, .236]$

$.14(.13) \quad[-.078, .445]$

$-.07(.06) \quad[-.224, .023]$

$-.05(.03) \quad[-.149,-.003]^{*}$

$-.08(.06) \quad[-.230,-.009]^{*}$

$-.06(.12) \quad[-.304, .183]$

$.003(.05) \quad[-.100, .110]$

$.03(.03) \quad[-.015, .106]$

$.04(.04) \quad[-.019, .147]$

$.34(.13) \quad[.129, .650]^{*}$

$-.10(.06) \quad[-.263,-.020]^{*}$

$-.07(.05) \quad[-.181,-.019]^{*}$

$-.11(.07) \quad[-.282,-.022]^{*}$ 
Shame/Embarrassment - Supportive Emotion Socialization

Negative emotionality $>$ Attributions of Fear $>$ Supportive $\quad-.09(.15) \quad[-.411, .185]$

Soothability $>$ Attributions of Fear $>$ Supportive $\quad-.03(.08) \quad[-.177, .120]$

Attention focusing $>$ Attributions of Fear $>$ Supportive $\quad .03(.04) \quad[-.019, .136]$

Inhibitory control $>$ Attributions of Fear $>$ Supportive $\quad .03(.05) \quad[-.051, .160]$

Note. Unstandardized parameter estimates are followed by the Bootstrapped standard errors (SE) in parentheses. The $95 \%$ bootstrapped lower limit confidence interval (LLCI) and upper limit confidence interval (ULCI) for each indirect effect is in brackets. * Indicates the indirect effect does not contain zero, which supports the hypothesized indirect effect. 
Table 13

Research Question 2: Predictors of Maternal-Reported Attributions of Sadness and Nonsupportive Emotion Socialization Responses to Sadness - Sample B

\begin{tabular}{|c|c|c|c|c|}
\hline \multirow[b]{3}{*}{ Predictor } & \multicolumn{4}{|c|}{ Outcome } \\
\hline & \multicolumn{2}{|c|}{$\begin{array}{l}\text { Attributions of } \\
\text { Sadness }\end{array}$} & \multicolumn{2}{|c|}{$\begin{array}{c}\text { Nonsupportive } \\
\text { Responses to } \\
\text { Sadness }\end{array}$} \\
\hline & $R^{2}$ & $\beta$ & $R^{2}$ & $\beta$ \\
\hline \multicolumn{5}{|l|}{ Block 1 - Main Effects and Controls (if any) } \\
\hline Negative Emotionality & $.19 * * *$ & $-.84 * * *$ & $.15 * *$ & .24 \\
\hline Soothability & $.18 * *$ & $.47 * * *$ & $.17 * *$ & $-.27 * *$ \\
\hline Attention Focusing & $.09 * *$ & $.24 *$ & $.17 * *$ & $-.18 * *$ \\
\hline Inhibitory Control & $.05^{\dagger}$ & $.28 *$ & $.13 * *$ & -.18 \\
\hline Attributions of Sadness - Negative Emotionality & - & - & - & $-.25 *$ \\
\hline Attributions of Sadness - Soothability & - & - & - & -.15 \\
\hline Attributions of Sadness - Attention Focusing & - & - & - & $-.18 *$ \\
\hline Attributions of Sadness - Inhibitory Control & - & - & - & $-.22 *$ \\
\hline Family Income - Negative Emotionality & - & - & - & $.04 * *$ \\
\hline Family Income - Soothability & - & - & - & $.05 * *$ \\
\hline Family Income - Attention Focusing & - & - & - & $.04 *$ \\
\hline Family Income - Inhibitory Control & - & - & - & $.04 *$ \\
\hline$n$ & 123 & & 123 & \\
\hline
\end{tabular}

Note. ${ }^{*} p<.05 . * * p<.01 . * * * p<.001 . \dagger$ trend. The PROCESS macro places all predictors, including control variables, within a single block. 
Table 14

Research Question 2: Predictors of Maternal-Reported Attributions of Sadness and Supportive Emotion Socialization Responses to Sadness - Sample B

Outcome

\begin{tabular}{|c|c|c|c|c|}
\hline \multirow[b]{2}{*}{ Predictor } & \multicolumn{2}{|c|}{$\begin{array}{l}\text { Attributions } \\
\text { of Sadness }\end{array}$} & \multicolumn{2}{|c|}{$\begin{array}{l}\text { Supportive } \\
\text { Responses } \\
\text { to Sadness }\end{array}$} \\
\hline & $R^{2}$ & $\beta$ & $R^{2}$ & $\beta$ \\
\hline \multicolumn{5}{|l|}{ Block 1 - Main Effects and Controls (if any) } \\
\hline Negative Emotionality & $.18^{* * *}$ & $-.82 * * *$ & $.10^{* *}$ & $-.51 *$ \\
\hline Soothability & $.18 * * *$ & $.46 * * *$ & $.14^{* *}$ & $.39 * *$ \\
\hline Attention Focusing & $.08 * *$ & $.23 * *$ & $.12 * *$ & $.26^{* *}$ \\
\hline Inhibitory Control & $.04^{*}$ & $.27 *$ & $.08^{*}$ & $.31 *$ \\
\hline Attributions of Sadness - Negative Emotionality & - & - & - & .13 \\
\hline Attributions of Sadness - Soothability & - & - & - & .05 \\
\hline Attributions of Sadness - Attention Focusing & - & - & - & .09 \\
\hline Attributions of Sadness - Inhibitory Control & - & - & - & .14 \\
\hline$n$ & 123 & & 123 & \\
\hline
\end{tabular}

Note. $* p<.05 . * * p<.01 . * * * p<.001 . \dagger$ trend. The PROCESS macro places all predictors, including control variables, within a single block. 
Table 15

Research Question 2: Predictors of Maternal-Reported Attributions of Anger and Nonsupportive Emotion Socialization Responses to Anger - Sample B

Outcome

\begin{tabular}{|c|c|c|c|c|}
\hline \multirow[b]{2}{*}{ Predictor } & \multicolumn{2}{|c|}{$\begin{array}{c}\text { Attributions } \\
\text { of Anger }\end{array}$} & \multicolumn{2}{|c|}{$\begin{array}{c}\text { Nonsupportive } \\
\text { Responses } \\
\text { to Anger }\end{array}$} \\
\hline & $R^{2}$ & $\beta$ & $R^{2}$ & $\beta$ \\
\hline \multicolumn{5}{|l|}{ Block 1 - Main Effects and Controls (if any) } \\
\hline Negative Emotionality & $.27 * * *$ & $-.1 .25 * * *$ & $.08 * *$ & .22 \\
\hline Soothability & $.29 * * *$ & $.73 * * *$ & $.08 * *$ & -.24 \\
\hline Attention Focusing & $.08 * *$ & $.27 * *$ & $.07 *$ & -.12 \\
\hline Inhibitory Control & $.06 * *$ & $.39 * *$ & $.07 *$ & -.17 \\
\hline Attributions of Anger - Negative Emotionality & - & - & - & $-.22 *$ \\
\hline Attributions of Anger - Soothability & - & - & - & -.14 \\
\hline Attributions of Anger - Attention Focusing & - & - & - & $-.20 *$ \\
\hline Attributions of Anger - Inhibitory Control & - & - & - & $-.20 *$ \\
\hline$n$ & 123 & & 123 & \\
\hline
\end{tabular}

Note. $* p<.05 . * * p<.01 . * * * p<.001 . \dagger$ trend. The PROCESS macro places all predictors, including control variables, within a single block. 
Table 16

Research Question 2: Predictors of Maternal Attributions of Anger and

Supportive Emotion Socialization Responses to Anger - Sample B

Outcome

\begin{tabular}{|c|c|c|c|c|}
\hline \multirow[b]{2}{*}{ Predictor } & \multicolumn{2}{|c|}{$\begin{array}{l}\text { Attributions } \\
\text { of Anger }\end{array}$} & \multicolumn{2}{|c|}{$\begin{array}{l}\text { Supportive } \\
\text { Responses } \\
\text { to Anger }\end{array}$} \\
\hline & $R^{2}$ & $\beta$ & $R^{2}$ & $\beta$ \\
\hline \multicolumn{5}{|l|}{ Block 1 - Main Effects and Controls (if any) } \\
\hline Negative Emotionality & $.26 * * *$ & $-1.25 * * *$ & $.19 * * *$ & -.42 \\
\hline Soothability & $.29 * * *$ & $.73 * * *$ & $.17 * *$ & $.43 * *$ \\
\hline Attention Focusing & $.08 * *$ & $.28 * *$ & $.14 * *$ & .16 \\
\hline Inhibitory Control & $.07 *$ & $.41 * *$ & $.16^{* *}$ & $.39 *$ \\
\hline Attributions of Anger - Negative Emotionality & - & - & - & $.26^{*}$ \\
\hline Attributions of Anger - Soothability & - & - & - & .11 \\
\hline Attributions of Anger - Attention Focusing & - & - & - & $.23^{*}$ \\
\hline Attributions of Anger - Inhibitory Control & - & - & - & $.20^{\dagger}$ \\
\hline Child Age - Negative Emotionality & - & - & - & $-.41 * *$ \\
\hline Child Age - Soothability & - & - & - & $-.31 * *$ \\
\hline Child Age - Attention Focusing & - & - & - & $-.35 * *$ \\
\hline Child Age - Inhibitory Control & - & - & - & $-.36^{* *}$ \\
\hline$n$ & 123 & & 123 & \\
\hline
\end{tabular}

Note. $* p<.05 . * * p<.01 . * * * p<.001 . \dagger$ trend. The PROCESS macro places all predictors, including control variables, within a single block. 
Table 17

Research Question 2: Predictors of Maternal Attributions of Fear and Nonsupportive Emotion Socialization Responses to Fear - Sample B

Outcome

\begin{tabular}{|c|c|c|c|c|}
\hline \multirow[b]{2}{*}{ Predictor } & \multicolumn{2}{|c|}{$\begin{array}{l}\text { Attributions } \\
\text { of Fear }\end{array}$} & \multicolumn{2}{|c|}{$\begin{array}{c}\text { Nonsupportive } \\
\text { Responses } \\
\text { to Fear }\end{array}$} \\
\hline & $R^{2}$ & $\beta$ & $R^{2}$ & $\beta$ \\
\hline \multicolumn{5}{|l|}{ Block 1 - Main Effects and Controls (if any) } \\
\hline Negative Emotionality & $.24 * * *$ & $-1.20 * * *$ & $.15^{* *}$ & $.68 * *$ \\
\hline Soothability & $.17 * * *$ & $.58 * * *$ & $.13 * *$ & $-.35 * *$ \\
\hline Attention Focusing & $.09 * *$ & $.30 * *$ & $.13 * *$ & $-.23 * *$ \\
\hline Inhibitory Control & $.08 * *$ & $.44 * *$ & $.09 * *$ & -.22 \\
\hline Attributions of Fear - Negative Emotionality & - & - & - & -.11 \\
\hline Attributions of Fear - Soothability & - & - & - & -.12 \\
\hline Attributions of Fear - Attention Focusing & - & - & - & $-.16^{\dagger}$ \\
\hline Attributions of Fear - Inhibitory Control & - & - & - & $-.19 *$ \\
\hline$n$ & 123 & & 123 & \\
\hline
\end{tabular}

Note. ${ }^{*} p<.05 . * * p<.01 . * * * p<.001 . \dagger$ trend. The PROCESS macro places all predictors, including control variables, within a single block. 
Table 18

Research Question 2: Predictors of Maternal Attributions of Fear and Supportive Emotion Socialization Responses to Fear - Sample B

\begin{tabular}{|c|c|c|c|c|}
\hline \multirow[b]{3}{*}{ Predictor } & \multicolumn{4}{|c|}{ Outcome } \\
\hline & \multicolumn{2}{|c|}{$\begin{array}{l}\text { Attributions } \\
\text { of Fear }\end{array}$} & \multicolumn{2}{|c|}{$\begin{array}{c}\text { Supportive } \\
\text { Responses } \\
\text { to Fear }\end{array}$} \\
\hline & $R^{2}$ & $\beta$ & $R^{2}$ & $\beta$ \\
\hline \multicolumn{5}{|l|}{ Block 1 - Main Effects and Controls (if any) } \\
\hline Negative Emotionality & $.25 * * *$ & $-1.24 * * *$ & .03 & -.22 \\
\hline Soothability & $.18 * * *$ & $.59 * * *$ & $.07 *$ & $.28 * *$ \\
\hline Attention Focusing & $.09 * *$ & $.31 * *$ & .01 & .01 \\
\hline Inhibitory Control & $.08 * *$ & $.47 * *$ & .02 & .05 \\
\hline Attributions of Fear - Negative Emotionality & - & - & - & .05 \\
\hline Attributions of Fear - Soothability & - & - & - & .005 \\
\hline Attributions of Fear - Attention Focusing & - & - & - & .09 \\
\hline Attributions of Fear - Inhibitory Control & - & - & - & .09 \\
\hline$n$ & 123 & & 123 & \\
\hline
\end{tabular}

Note. $* p<.05 . * * p<.01 . * * * p<.001 . \dagger$ trend. The PROCESS macro places all predictors, including control variables, within a single block. 


\section{Table 19}

Research Question 2: Predictors of Maternal Attributions of Shame/Embarrassment and Nonsupportive Emotion Socialization Responses to Shame/Embarrassment - Sample B

\section{Outcome}

\begin{tabular}{|c|c|c|c|c|}
\hline \multirow[b]{2}{*}{ Predictor } & \multicolumn{2}{|c|}{$\begin{array}{c}\text { Attributions } \\
\text { of Shame/Emb. }\end{array}$} & \multicolumn{2}{|c|}{$\begin{array}{l}\text { Nonsupportive } \\
\text { Responses } \\
\text { to Shame/Emb. }\end{array}$} \\
\hline & $R^{2}$ & ß & $R^{2}$ & $\beta$ \\
\hline \multicolumn{5}{|l|}{ Block 1 - Main Effects and Controls (if any) } \\
\hline Negative Emotionality & $.24 * * *$ & $-1.62 * * *$ & $.14^{* *}$ & .29 \\
\hline Soothability & $.22 * * *$ & $.62 * * *$ & $.18 * * *$ & $-.41 * *$ \\
\hline Attention Focusing & $.09 * *$ & $.29 * *$ & $.14 * *$ & $-.17 *$ \\
\hline Inhibitory Control & $.06 * *$ & $.39 * *$ & $.12 * *$ & -.17 \\
\hline Attributions of Shame/Emb. - Negative Emotionality & - & - & - & $-.29 * *$ \\
\hline Attributions of Shame/Emb. - Soothability & - & - & - & -.17 \\
\hline Attributions of Shame/Emb. - Attention Focusing & - & - & - & $-.26 * *$ \\
\hline Attributions of Shame/Emb. - Inhibitory Control & - & - & - & $-.29 * *$ \\
\hline$n$ & 123 & & 123 & \\
\hline
\end{tabular}

Note. Shame/Emb. $=$ Shame/Embarrassment. $* p<.05 . * * p<.01 . * * * p<.001 . \dagger$ trend. The PROCESS macro places all predictors, including control variables, within a single block. 
Table 20

Research Question 2: Predictors of Maternal Attributions of Shame/Embarrassment and Supportive Emotion Socialization Responses to Shame/Embarrassment - Sample B

\begin{tabular}{|c|c|c|c|c|}
\hline \multirow[b]{3}{*}{ Predictor } & \multicolumn{4}{|c|}{ Outcome } \\
\hline & \multicolumn{2}{|c|}{$\begin{array}{c}\text { Attributions } \\
\text { of Shame/Emb. }\end{array}$} & \multicolumn{2}{|c|}{$\begin{array}{c}\text { Supportive } \\
\text { Responses } \\
\text { to Shame/Emb. }\end{array}$} \\
\hline & $R^{2}$ & $\beta$ & $R^{2}$ & $\beta$ \\
\hline \multicolumn{5}{|l|}{ Block 1 - Main Effects and Controls (if any) } \\
\hline Negative Emotionality & $.23 * * *$ & $-1.12 * * *$ & .02 & -.30 \\
\hline Soothability & $.19 * * *$ & $.59 * * *$ & $.06^{*}$ & $.45^{* *}$ \\
\hline Attention Focusing & $.09 * *$ & $.29 * *$ & .01 & .02 \\
\hline Inhibitory Control & $.07 * *$ & $.42 * *$ & .03 & .26 \\
\hline Attributions of Shame/Emb. - Negative Emotionality & - & - & - & .08 \\
\hline Attributions of Shame/Emb. - Soothability & - & - & - & -.04 \\
\hline Attributions of Shame/Emb. - Attention Focusing & - & - & - & .11 \\
\hline Attributions of Shame/Emb. - Inhibitory Control & - & - & - & .07 \\
\hline$n$ & 123 & & 123 & \\
\hline
\end{tabular}

Note. Shame/Emb. $=$ Shame/Embarrassment. $* p<.05 . * * p<.01 . * * * p<.001 . \dagger$ trend. The PROCESS macro places all predictors, including control variables, within a single block. 
Table 21

Research Question 3: Demographic Predictors of Cognitive Reappraisal, Expressive Suppression, and Regulatory Emotional Self-Efficacy of Negative Emotions

\begin{tabular}{|c|c|c|c|c|c|c|}
\hline \multirow[b]{3}{*}{ Demographic Predictor } & \multicolumn{6}{|c|}{ Emotion Outcome } \\
\hline & \multicolumn{2}{|c|}{$\begin{array}{c}\text { Cognitive } \\
\text { Reappraisal }\end{array}$} & \multicolumn{2}{|c|}{$\begin{array}{l}\text { Expressive } \\
\text { Suppression }\end{array}$} & \multicolumn{2}{|c|}{$\begin{array}{c}\text { Regulatory } \\
\text { Emotional Self- } \\
\text { Efficacy }\end{array}$} \\
\hline & $\Delta R^{2}$ & $\beta$ & $\Delta R^{2}$ & $\beta$ & $\Delta R^{2}$ & $\beta$ \\
\hline Step 1 & .07 & & .08 & & .05 & \\
\hline Child Sex & & -.08 & & $-.24 *$ & & -.07 \\
\hline Child Age & & -.13 & & .10 & & .02 \\
\hline Maternal Race & & -.15 & & -.13 & & -.09 \\
\hline Maternal Education & & -.05 & & .05 & & -.18 \\
\hline Maternal Occupation Status & & .13 & & .02 & & -.12 \\
\hline Household Income & & -.04 & & -.05 & & .004 \\
\hline Total Adj. $R^{2}$ & .02 & & .03 & & .000 & \\
\hline$n$ & 123 & & 123 & & 123 & \\
\hline
\end{tabular}

Note. $* p<.05 . * * p<.01 . * * * p<.001 . \dagger$ trend. 


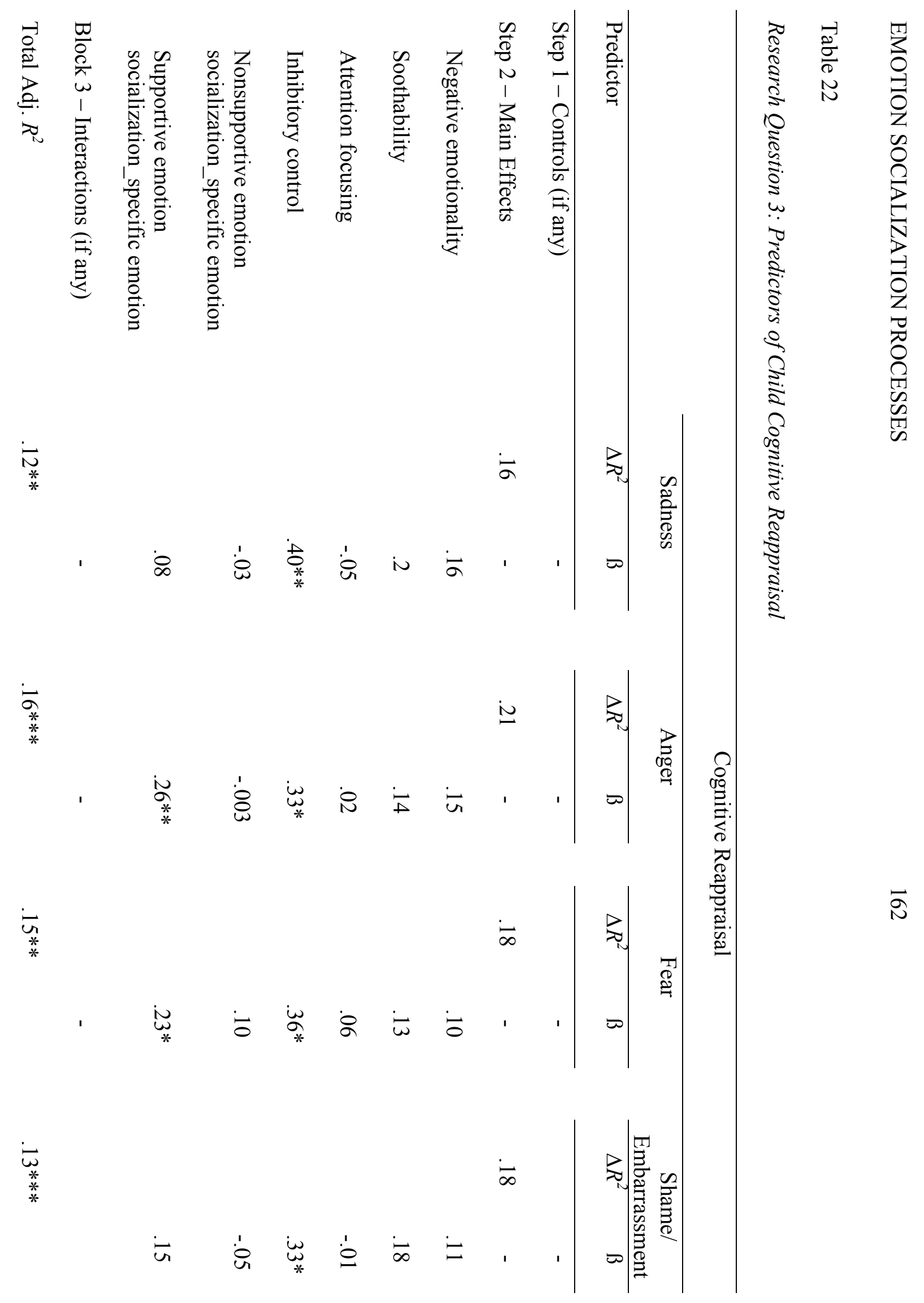




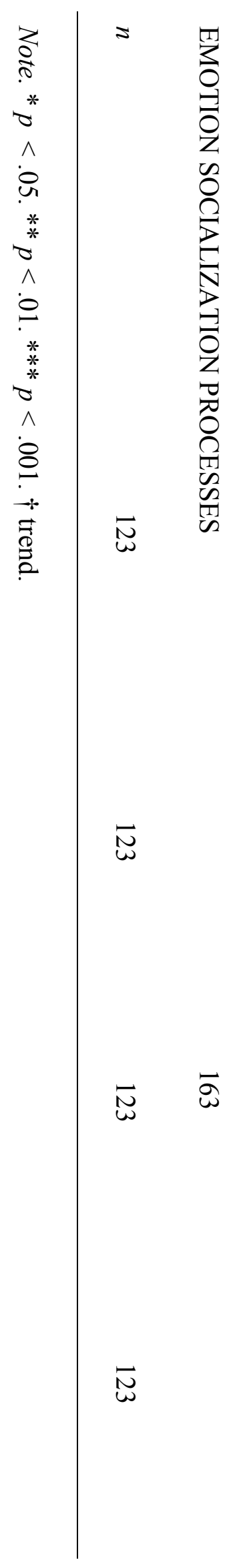




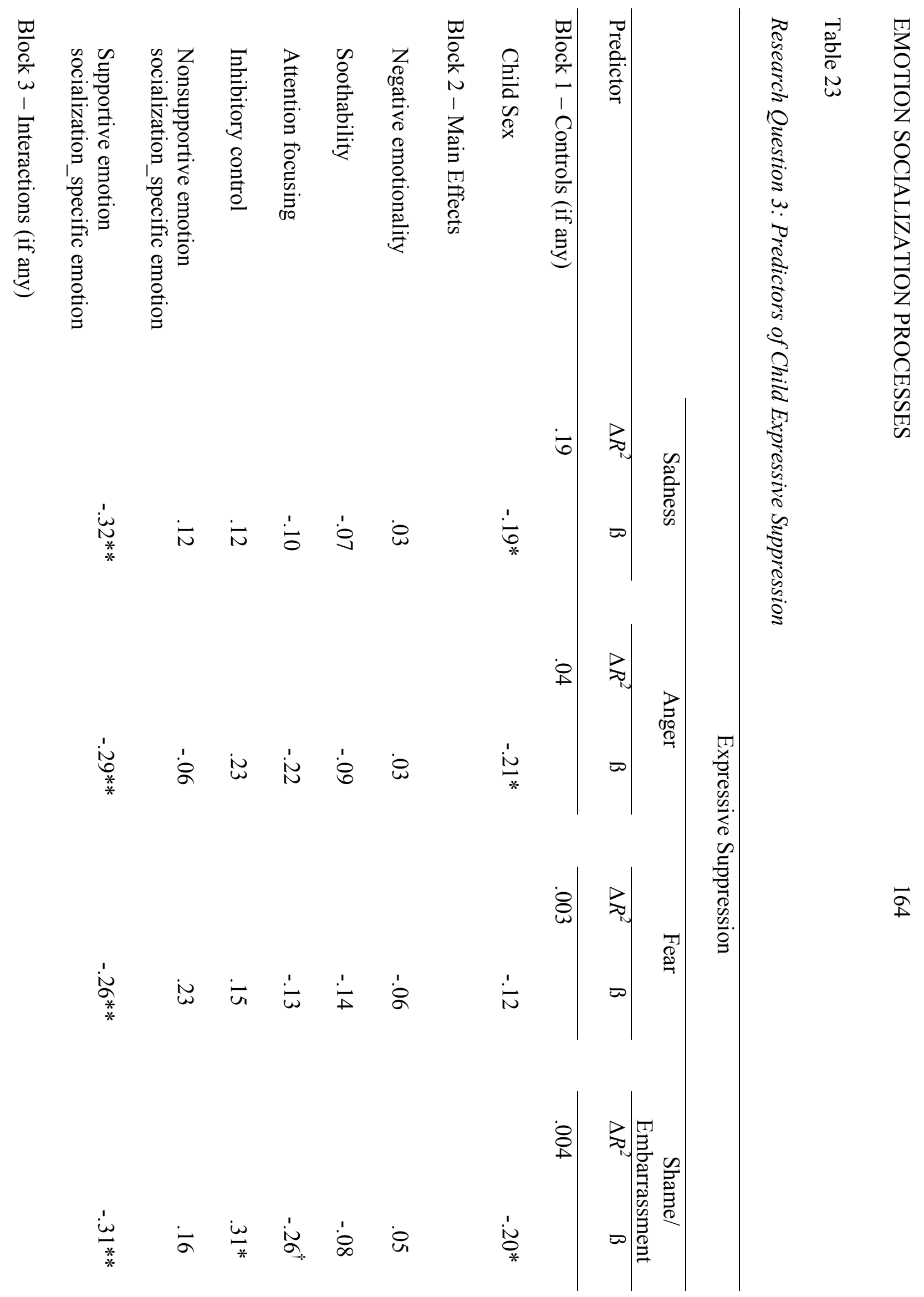




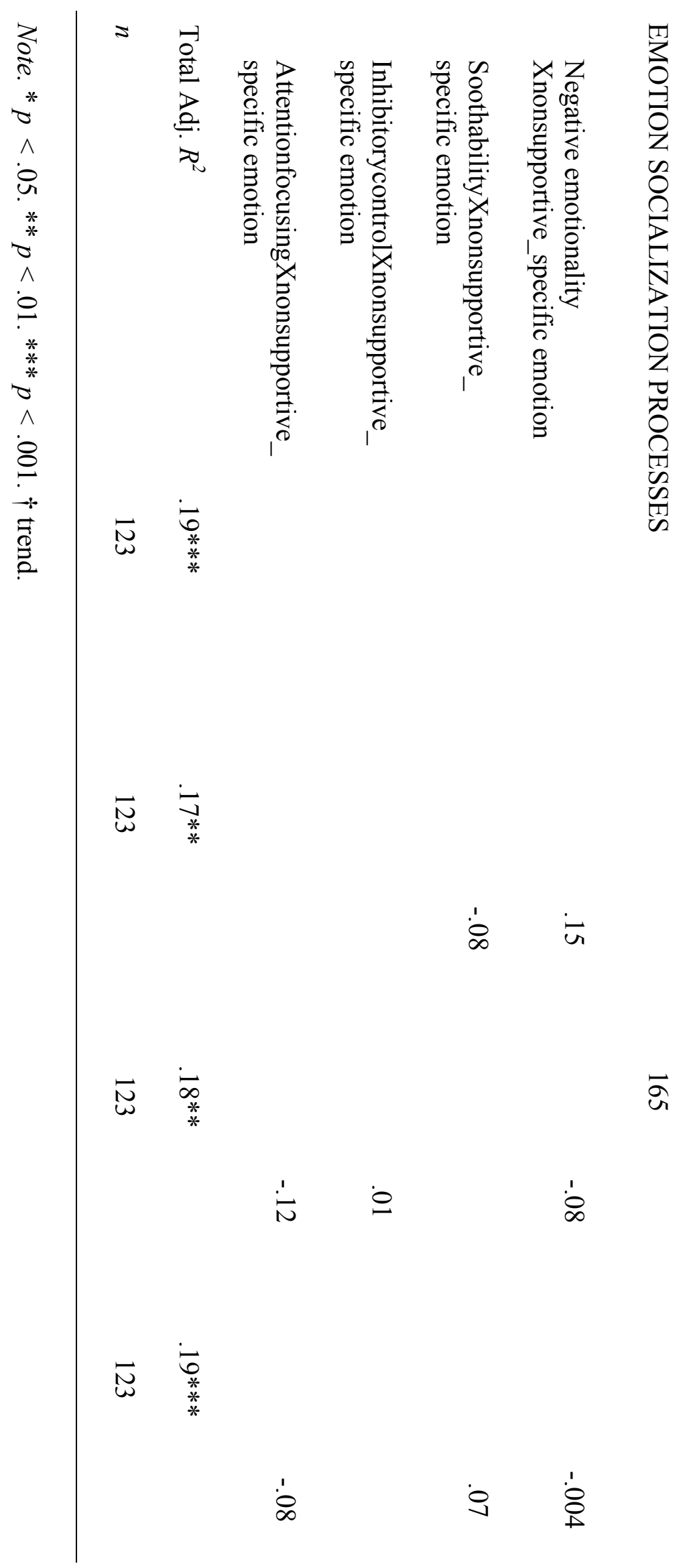



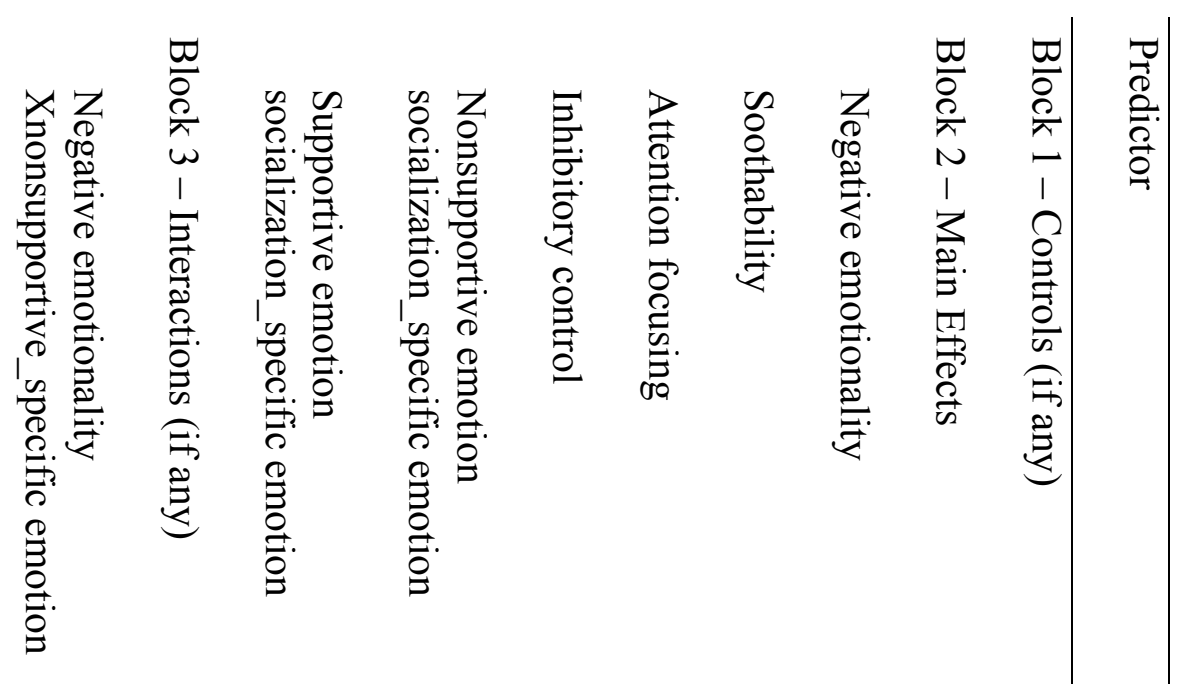

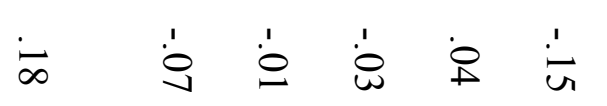

8

草

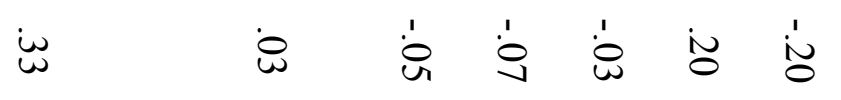

㽜

岀守守它它

:

$\dot{\infty}$

$\bar{u}$ 


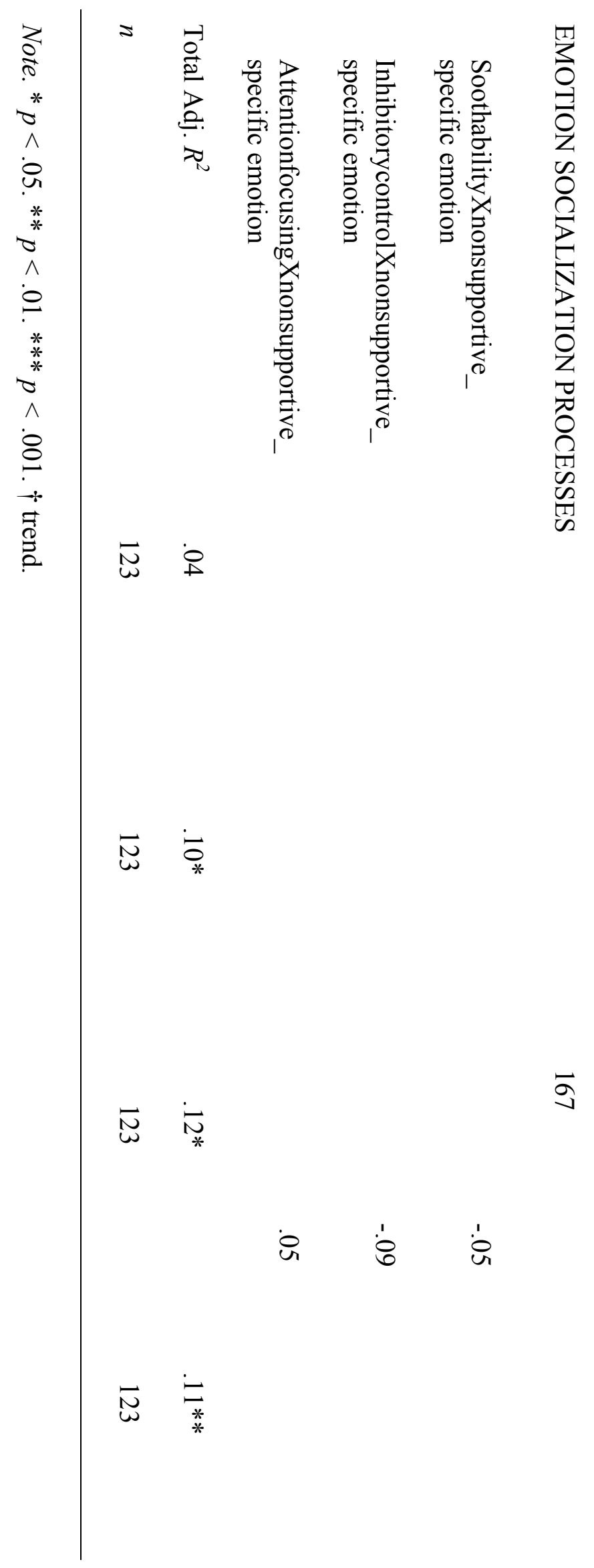




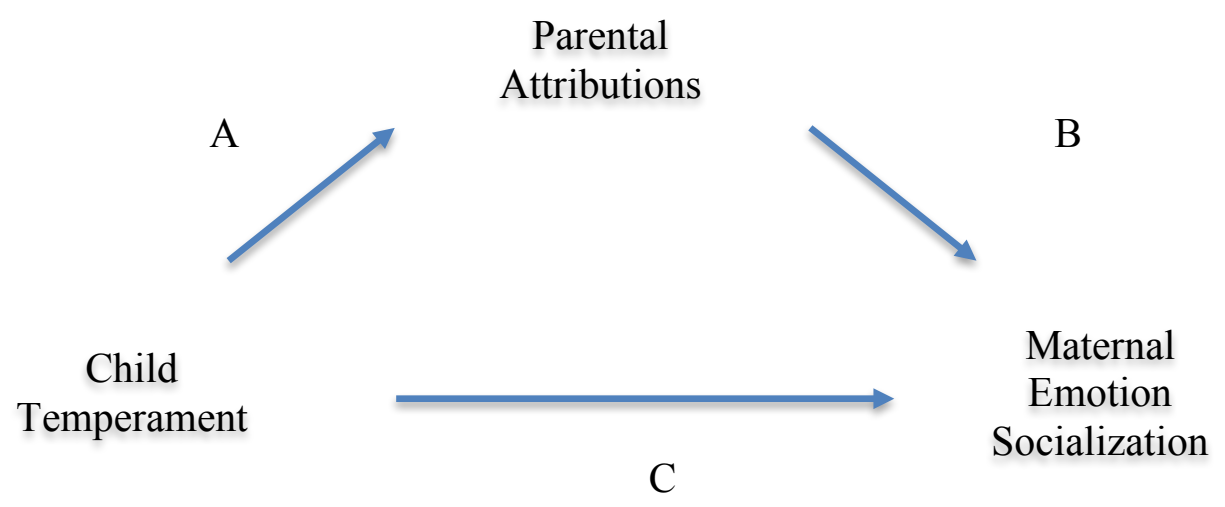

Figure 1. Conceptual model for research question two: indirect effects of child temperament. 


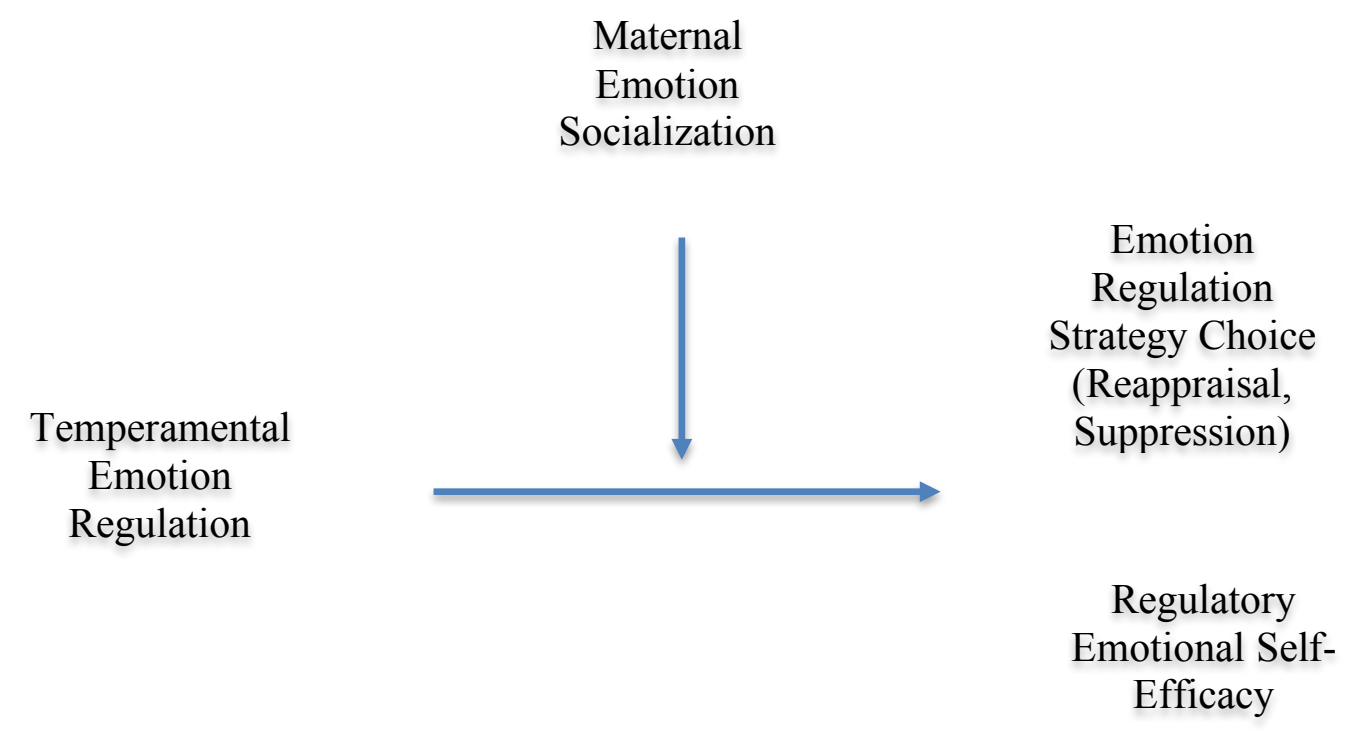

Figure 2. Conceptual model for research question three: moderation. 


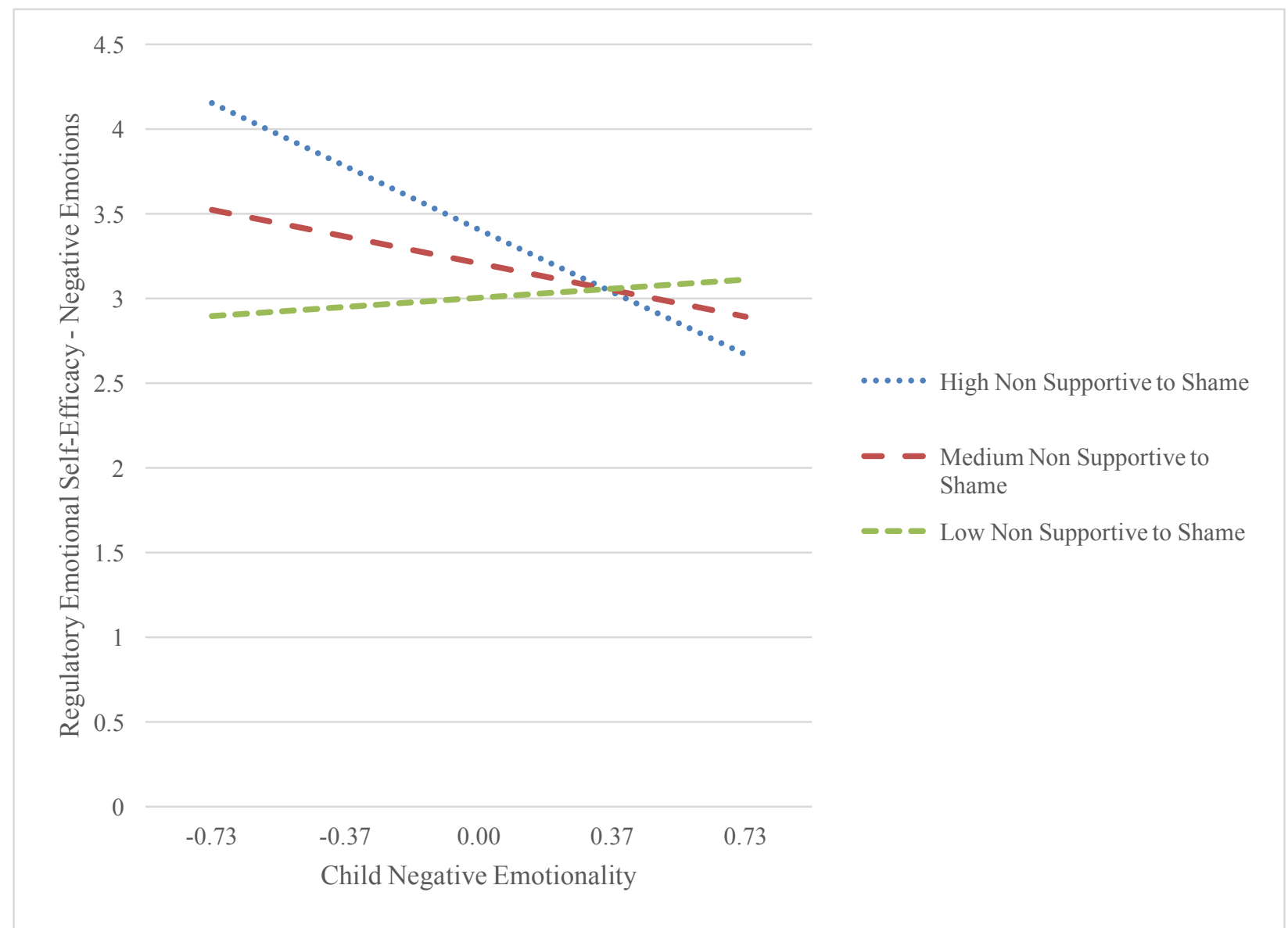

Figure 3. Maternal nonsupportive emotion socialization to shame as significant moderator between child negative emotionality and regulatory emotional self-efficacy of negative emotions. 
Maternal Attributions of Sadness Displays - model significant Soothability $>$ Maternal Attributions (+ beta weight)

Maternal Attributions of Anger Displays - model significant Negative Emotionality $>$ Maternal Attributions (- beta weight) Soothability $>$ Maternal Attributions (+ beta weight) Attention Focusing $>$ Maternal Attributions (- beta weight) Inhibitory Control > Maternal Attributions (+ beta weight)

Maternal Attributions of Fear Displays - model significant Soothability $>$ Maternal Attributions (+ beta weight) Inhibitory Control > Maternal Attributions (+ beta weight) Maternal Attributions of Shame/Embarrassment Displays - model significant Soothability $>$ Maternal Attributions (+ beta weight) Inhibitory Control $>$ Maternal Attributions (+ beta weight) Figure 4. Research question 1: Summary of significant findings. 
Nonsupportive Emotion Socialization Responses to Sadness

Indirect effect of attributions of sadness displays for negative emotionality, soothability, attention focusing, and inhibitory control models (temperament $>$ maternal attributions $>$ nonsupportive responses to sadness).

Effect of family income on nonsupportive responses to sadness for all four temperament dimensions (all + beta weights)

Supportive Emotion Socialization Responses to Sadness

No indirect effects of maternal attributions of sadness displays for any of the four temperamental trait models.

Nonsupportive Emotion Socialization Responses to Anger

Indirect effect of maternal attributions of anger displays for negative emotionality, attention focusing, and inhibitory control models (temperament $>$ maternal attributions $>$ nonsupportive responses to anger).

Supportive Emotion Socialization Responses to Anger

Indirect effect of maternal attributions of anger displays for negative emotionality and attention focusing models (temperament $>$ maternal attributions $>$ nonsupportive responses to anger).

Effect of child age on supportive responses to anger for negative emotionality, soothability, and attention focusing models (all - beta weights).

Nonsupportive Emotion Socialization Responses to Fear

Indirect effect of maternal attributions of fear for attention focusing and inhibitory control models (temperament $>$ maternal attributions $>$ nonsupportive responses to fear).

Supportive Emotion Socialization Responses to Fear

No indirect effects of maternal attributions of fear displays for any of the four temperamental trait models.

Nonsupportive Emotion Socialization Responses to Shame/Embarrassment

Indirect effect of attributions of sadness displays for negative emotionality, soothability, attention focusing, and inhibitory control models (temperament $>$ maternal attributions $>$ nonsupportive responses to shame/embarrassment). 
Supportive Emotion Socialization Responses to Shame/Embarrassment

No indirect effects of maternal attributions of shame/embarrassment displays for any of the four temperament trait models.

Figure 5. Research question 2: Summary of significant findings. 
Cognitive Reappraisal in Relation to Emotion Socialization of Sadness Inhibitory Control > Cognitive Reappraisal (+ beta weight)

Cognitive Reappraisal in Relation to Emotion Socialization of Anger Inhibitory Control > Cognitive Reappraisal (+ beta weight) Supportive Responses to Anger $>$ Cognitive Reappraisal (+ beta weight)

Cognitive Reappraisal in Relation to Emotion Socialization of Fear Inhibitory Control > Cognitive Reappraisal (+ beta weight) Supportive Responses to Fear $>$ Cognitive Reappraisal (+ beta weight)

Cognitive Reappraisal in Relation to Emotion Socialization of Shame/Embarrassment Inhibitory Control > Cognitive Reappraisal (+ beta weight)

Expressive Suppression in Relation to Emotion Socialization of Sadness Child Sex $>$ Expressive Suppression (- beta weight; $0=$ male, $1=$ female) Supportive Responses to Sadness $>$ Expressive Suppression (- beta weight) Expressive Suppression in Relation to Emotion Socialization of Anger Child Sex $>$ Expressive Suppression (- beta weight; $0=$ male, $1=$ female) Supportive Responses to Anger > Expressive Suppression (- beta weight) Expressive Suppression in Relation to Emotion Socialization of Fear Supportive Responses to Fear $>$ Expressive Suppression (- beta weight) Expressive Suppression in Relation to Emotion Socialization of Shame/Embarrassment Child Sex $>$ Expressive Suppression (- beta weight; $0=$ male, $1=$ female) Inhibitory Control $>$ Expressive Suppression (+ beta weight) Supportive Responses to Shame/Embarrassment > Expressive Suppression (- beta weight) 
Regulatory Emotional Self-Efficacy of Negative Emotions in Relation to Emotion Socialization of Sadness

Model not significant

Regulatory Emotional Self-Efficacy of Negative Emotions in Relation to Emotion Socialization of Anger

Supportive Responses to Anger > Regulatory Emotional Self-Efficacy (+ beta weight)

Regulatory Emotional Self-Efficacy of Negative Emotions in Relation to Emotion Socialization of Fear

Model significant, none of predictors were significant.

Regulatory Emotional Self-Efficacy of Negative Emotions in Relation to Emotion Socialization of Shame/Embarrassment

Nonsupportive Responses to Shame/Embarrassment > Regulatory Emotional Self-Efficacy (- beta weight)

Negative Emotionality x Nonsupportive Responses to Shame/Embarrassment $>$ Regulatory Emotional Self-Efficacy. Likely due to Type 1 error.

Figure 6. Research question 3: Summary of significant findings. 
Appendix 1

Demographic Questionnaire 
Demographic Questionnaire

\section{What is today's date?}

\section{Your Gender:}

Male

Female

Your Relation to Child:

Mother

Father

Stepmother

Stepfather

Grandmother

Grandfather

Other (please specify):

Your Age:

\section{Your Race:}

American Indian/Alaska Native

Asian

Black or African American

Native Hawaiian or Other Pacific Islander

White or Caucasian

Bi- or Multi-racial (please specify):

Other (please specify):

\section{Your Ethnicity:}

Hispanic or Latino

Not Hispanic or Latino

Your Education level (please select highest level)

Elementary School

High School

Vocational School

Some College

University Degree

Some Graduate School

Master's Degree

Doctoral Degree 


\section{Your Occupation Status}

Employed full-time

Employed part-time

Not employed outside of home

Retired

Unemployed and seeking employment

Other (specify)

Where do you currently reside?

-Northeast, Division 1 (New England) Maine, New Hampshire, Vermont, Massachusetts, Rhode Island, Connecticut

-Northeast, Division 2 (Mid-Atlantic) New York, Pennsylvania, New Jersey

-Midwest, Division 3 (East North Central) Wisconsin, Michigan, Illinois, Indiana, Ohio

-Midwest, Division 4 (West North Central) Missouri, North Dakota, South Dakota, Nebraska, Kansas, Minnesota, Iowa

-South, Division 5 (South Atlantic) Delaware, Maryland, District of Columbia, Virginia, West Virginia, North Carolina, South Carolina, Georgia, Florida

-South, Division 6 (East South Central) Kentucky, Tennessee, Mississippi, Alabama

-South, Division 7 (West South Central) Oklahoma, Texas, Arkansas, Louisiana

-West, Division 8 (Mountain) Idaho, Montana, Wyoming, Nevada, Utah, Colorado, Arizona, New Mexico

-West, Division 9 (Pacific) Alaska, Washington, Oregon, California, Hawaii

-Do not reside in the United States

\section{Household Income:}

$$
\begin{aligned}
& \text { Less than } \$ 10,000 \\
& \$ 10,000-\$ 25,000 \\
& \$ 25,000-\$ 50,000 \\
& \$ 75,000-\$ 100,000 \\
& \$ 100,000-\$ 150,000 \\
& \text { Greater than } \$ 150,000 \\
& \text { Prefer not to answer }
\end{aligned}
$$

Please answer the following questions about the child you will complete the rest of the questionnaires about (child that is 7-11 years of age). If you have more than one child in this age range, please choose the younger child and remember to complete all questionnaires about the same child.

Child's Initials:

Child's Age:

\section{Child's Gender:}

Male

Female 


\section{Child's Race:}

American Indian/Alaska Native

Asian

Black or African American

Native Hawaiian or Other Pacific Islander

White or Caucasian

Bi- or Multi-racial (please specify):

Other (please specify):

\section{Child's Ethnicity:}

Hispanic or Latino

Not Hispanic or Latino

\section{Child's biological relation to you:}

Biological

Adopted

Foster

If adopted or foster, please provide the age child was adopted or fostering began:

Is your child a twin?

Yes

No

If yes, identical or fraternal?

Where does your child go to school?

Public School

Private School

Home School

Charter School

What is the last grade your child completed in school?

Kindergarten

$1^{\text {st }}$

$2^{\text {nd }}$

$3^{\text {rd }}$

$4^{\text {th }}$

$5^{\text {th }}$

$6^{\text {th }}$

$7^{\text {th }}$ 
Other:

What grade is your child currently enrolled?

Kindergarten

$1^{\text {st }}$

$2^{\text {nd }}$

$3^{\text {rd }}$

$4^{\text {th }}$

$5^{\text {th }}$

$6^{\text {th }}$

$7^{\text {th }}$

Other:

Is s/he currently enrolled in:

Elementary school

Middle school

N/A

Where does child reside:

With me $100 \%$ of the time

With me $75 \%-99 \%$ of the time

With me $50 \%-74 \%$ of the time

With me $25 \%-49 \%$ of the time

With me less than $25 \%$ of the time

If the child resides with you less than $100 \%$ of the time, who else does s/he life with?

How many other children do you have?

How many other children reside with the child you are reporting about? 


\section{Child's birth order:}

First

Second

Third

Fourth

Fifth

Sixth

Other (please specify):

Does your child have any serious medical problems?

Yes

No

If yes, please specify:

Has your child been diagnosed with a developmental delay?

Yes

No

If yes, please specify: 
Appendix 2

Emotion Regulation Checklist 
Emotion Regulation Checklist

\begin{tabular}{|c|c|c|c|c|}
\hline & $\begin{array}{c}1 \\
\text { Never }\end{array}$ & $\begin{array}{c}2 \\
\text { Sometimes }\end{array}$ & $\begin{array}{c}3 \\
\text { Often }\end{array}$ & $\begin{array}{c}4 \\
\text { Always }\end{array}$ \\
\hline My child is a cheerful child. & & & & \\
\hline $\begin{array}{l}\text { My child exhibits wide mood swings (child's } \\
\text { emotional state is difficult to anticipate because } \\
\text { h/she moves quickly from positive to negative } \\
\text { moods. }\end{array}$ & & & & \\
\hline $\begin{array}{l}\text { My child responds positively to neutral or friendly } \\
\text { overtures by adults. }\end{array}$ & & & & \\
\hline $\begin{array}{l}\text { My child transitions well from one activity to } \\
\text { another; does not become anxious, angry, } \\
\text { distressed, or overly excited when moving from } \\
\text { one activity to another. }\end{array}$ & & & & \\
\hline $\begin{array}{l}\text { My child can recover quickly from episodes of } \\
\text { upset or distress (e.g., does not pout or remain } \\
\text { sullen, anxious, or sad after emotionally } \\
\text { distressing events) }\end{array}$ & & & & \\
\hline My child is easily frustrated. & & & & \\
\hline $\begin{array}{l}\text { My child responds positively to neutral or friendly } \\
\text { overtures by peers. }\end{array}$ & & & & \\
\hline My child is prone to angry outbursts. & & & & \\
\hline My child is able to delay gratification. & & & & \\
\hline $\begin{array}{l}\text { My child takes pleasure in the distress of others } \\
\text { (e.g., laughs when another person gets hurt or } \\
\text { punished; enjoys teasing others) }\end{array}$ & & & & \\
\hline $\begin{array}{l}\text { My child can modulate excitement in emotionally } \\
\text { arousing situations (e.g., does not get "carried } \\
\text { away" in high-energy play situations, or overly } \\
\text { excited in inappropriate contexts. }\end{array}$ & & & & \\
\hline My child is whiny or clingy with adults. & & & & \\
\hline $\begin{array}{l}\text { My child is prone to disruptive outbursts of } \\
\text { energy and exuberance. }\end{array}$ & & & & \\
\hline $\begin{array}{l}\text { My child responds angrily to limit-setting by } \\
\text { adults. }\end{array}$ & & & & \\
\hline
\end{tabular}




\begin{tabular}{|c|c|c|c|c|}
\hline & $\begin{array}{c}1 \\
\text { Never }\end{array}$ & $\begin{array}{c}2 \\
\text { Sometimes }\end{array}$ & $\begin{array}{c}3 \\
\text { Often }\end{array}$ & $\begin{array}{c}4 \\
\text { Always }\end{array}$ \\
\hline $\begin{array}{l}\text { My child can say when } \mathrm{h} / \text { she is feeling sad, angry } \\
\text { or mad, fearful or afraid. }\end{array}$ & & & & \\
\hline My child seems sad or listless. & & & & \\
\hline $\begin{array}{l}\text { My child is overly exuberant when attempting to } \\
\text { engage others in play. }\end{array}$ & & & & \\
\hline $\begin{array}{l}\text { My child displays flat affect (expression is vacant } \\
\text { and inexpressive; child seems emotionally } \\
\text { absent). }\end{array}$ & & & & \\
\hline $\begin{array}{l}\text { My child responds negatively to neutral or } \\
\text { friendly overtures by peers (e.g., may speak in } \\
\text { angry tone of voice or respond fearfully). }\end{array}$ & & & & \\
\hline My child is impulsive. & & & & \\
\hline $\begin{array}{l}\text { My child is empathic toward others; shows } \\
\text { concern when others are upset or distressed. }\end{array}$ & & & & \\
\hline $\begin{array}{l}\text { My child displays exuberance that others find } \\
\text { intrusive or disruptive. }\end{array}$ & & & & \\
\hline $\begin{array}{l}\text { My child displays appropriate negative emotions } \\
\text { (anger, fear, frustration, distress) in response to } \\
\text { hostile, aggressive, or intrusive acts by peers. }\end{array}$ & & & & \\
\hline $\begin{array}{l}\text { My child displays negative emotions when } \\
\text { attempting to engage others in play. }\end{array}$ & & & & \\
\hline
\end{tabular}

Shields, A. M., \& Cicchetti, D. (1995, March). The development of an emotion regulation assessment battery: Reliability and validity among at-risk grade-school children. Poster session presented at the biennial meeting of the Society for Research in Child Development, Indianapolis, IN. 
Appendix 3

Maternal Attributions of Negative Emotion Expressions

Protocol Based on Coplan, Hastings, Lagacé-Séguin, \& Moulton, 2002 
Maternal Attributions of Negative Emotion Expressions

Protocol Based on Coplan, Hastings, Lagacé-Séguin, \& Moulton, 2002

Think of a time when your child expressed sadness. To what extent to did each of these factors influence your child's expression of sadness?

\begin{tabular}{|c|c|c|c|c|}
\hline $\begin{array}{c}1 \\
\text { My child will keep } \\
\text { acting this way }\end{array}$ & 2 & 3 & 4 & $\begin{array}{l}5 \\
\text { This is a stage that } \\
\text { will pass }\end{array}$ \\
\hline $\begin{array}{l}1 \\
\text { My child expressed } \\
\text { the emotion like } \\
\text { that on purpose }\end{array}$ & 2 & 3 & 4 & $\begin{array}{c}5 \\
\text { My child didn't mean } \\
\text { to express the } \\
\text { emotion in that way }\end{array}$ \\
\hline $\begin{array}{l}1 \\
\text { This is just like how } \\
\text { my child behaves }\end{array}$ & 2 & 3 & 4 & $\begin{array}{c}5 \\
\text { My child never } \\
\text { expresses this } \\
\text { emotion in this way }\end{array}$ \\
\hline $\begin{array}{c}1 \\
\text { Their expression of } \\
\text { the emotion was } \\
\text { due to my child's } \\
\text { personality }\end{array}$ & 2 & 3 & 4 & $\begin{array}{c}5 \\
\text { Their expression of } \\
\text { the emotion was due } \\
\text { to the situation }\end{array}$ \\
\hline
\end{tabular}

Think of a time when your child expressed anger. To what extent to did each of these factors influence your child's expression of anger?

\begin{tabular}{|c|c|c|c|c|}
\hline $\begin{array}{c}\text { My child will keep } \\
\text { acting this way }\end{array}$ & 2 & 3 & 4 & $\begin{array}{c}5 \\
\text { This is a stage that } \\
\text { will pass }\end{array}$ \\
\hline $\begin{array}{c}\text { My child expressed } \\
\text { the emotion like } \\
\text { that on purpose }\end{array}$ & 2 & 3 & 4 & $\begin{array}{c}5 \\
\text { My child didn't mean } \\
\text { to express the } \\
\text { emotion in that way }\end{array}$ \\
\hline $\begin{array}{c}\text { This is just like how } \\
\text { my child behaves }\end{array}$ & 2 & 3 & 4 & $\begin{array}{c}5 \\
\text { My child never } \\
\text { expresses this } \\
\text { emotion in this way }\end{array}$ \\
\hline $\begin{array}{c}\text { Their expression of } \\
\text { the emotion was } \\
\text { due to my child's } \\
\text { personality }\end{array}$ & 2 & 3 & 4 & $\begin{array}{c}5 \\
\text { Their expression of } \\
\text { the emotion was due } \\
\text { to the situation }\end{array}$ \\
\hline
\end{tabular}


Think of a time when your child expressed fear. To what extent to did each of these factors influence your child's expression of fear?

\begin{tabular}{|c|c|c|c|c|}
\hline $\begin{array}{c}1 \\
\text { My child will keep } \\
\text { acting this way }\end{array}$ & 2 & 3 & 4 & $\begin{array}{c}5 \\
\text { This is a stage that } \\
\text { will pass }\end{array}$ \\
\hline $\begin{array}{c}\text { My child expressed } \\
\text { the emotion like that } \\
\text { on purpose }\end{array}$ & 2 & 3 & 4 & $\begin{array}{c}5 \\
\text { My child didn't mean } \\
\text { to express the emotion } \\
\text { in that way }\end{array}$ \\
\hline $\begin{array}{c}\text { This is just like how } \\
\text { my child behaves }\end{array}$ & 2 & 3 & 4 & $\begin{array}{c}5 \\
\text { My child never } \\
\text { expresses this emotion } \\
\text { in this way }\end{array}$ \\
\hline $\begin{array}{c}\text { Their expression of } \\
\text { the emotion was due } \\
\text { to my child's } \\
\text { personality }\end{array}$ & 2 & 3 & 4 & $\begin{array}{c}5 \\
\text { Their expression of } \\
\text { the emotion was due } \\
\text { to the situation }\end{array}$ \\
\hline
\end{tabular}

Think of a time when your child expressed shame/embarrassment. To what extent to did each of these factors influence your child's expression of shame/embarrassment.

\begin{tabular}{|c|c|c|c|c|}
\hline $\begin{array}{c}1 \\
\text { My child will keep } \\
\text { acting this way }\end{array}$ & 2 & 3 & 4 & $\begin{array}{c}5 \\
\text { This is a stage that } \\
\text { will pass }\end{array}$ \\
\hline $\begin{array}{c}1 \\
\text { My child expressed } \\
\text { the emotion like that } \\
\text { on purpose }\end{array}$ & 2 & 3 & 4 & $\begin{array}{l}5 \\
\text { My child didn't mean } \\
\text { to express the emotion } \\
\text { in that way }\end{array}$ \\
\hline $\begin{array}{c}1 \\
\text { This is just like how } \\
\text { my child behaves }\end{array}$ & 2 & 3 & 4 & $\begin{array}{c}5 \\
\text { My child never } \\
\text { expresses this emotion } \\
\text { in this way }\end{array}$ \\
\hline $\begin{array}{c}1 \\
\text { Their expression of } \\
\text { the emotion was due } \\
\text { to my child's } \\
\text { personality }\end{array}$ & 2 & 3 & 4 & $\begin{array}{c}5 \\
\text { Their expression of } \\
\text { the emotion was due } \\
\text { to the situation }\end{array}$ \\
\hline
\end{tabular}


Coplan, R. J., Hastings, P. D., Lagacé-Séguin, D. G., \& Moulton, C. E. (2002). Authoritative and authoritarian mothers' parenting goals, attributions, and emotions across different childrearing contexts. Parenting, 2, 1-26. 


\author{
Appendix 4 \\ Emotion Regulation Questionnaire (Child Version)
}

Adapted from Gross \& John, 2003 
Emotion Regulation Questionnaire (Child Version)

Adapted from Gross \& John, 2003

\begin{tabular}{|c|c|c|c|c|c|}
\hline & $\begin{array}{l}1 \\
\text { Strongly } \\
\text { Disagree }\end{array}$ & $\begin{array}{c}2 \\
\text { Disagree }\end{array}$ & $\begin{array}{l}3 \\
\text { Half and } \\
\text { Half }\end{array}$ & $\begin{array}{c}4 \\
\text { Agree }\end{array}$ & $\begin{array}{c}5 \\
\text { Strongly } \\
\text { Agree }\end{array}$ \\
\hline $\begin{array}{l}\text { 1. When I want to feel happier, I } \\
\text { think about something different. }\end{array}$ & & & & & \\
\hline 2. I keep my feelings to myself. & & & & & \\
\hline $\begin{array}{l}\text { 3. When I want to feel less bad } \\
\text { (e.g., sad, angry, or worried), I } \\
\text { think about something different. }\end{array}$ & & & & & \\
\hline $\begin{array}{l}\text { 4. When I am feeling happy, I am } \\
\text { careful not to show it. }\end{array}$ & & & & & \\
\hline $\begin{array}{l}5 \text {. When I'm worried about } \\
\text { something, I make myself think } \\
\text { about it in a way that helps me feel } \\
\text { better. }\end{array}$ & & & & & \\
\hline $\begin{array}{l}\text { 6. I control my feelings by not } \\
\text { showing them. }\end{array}$ & & & & & \\
\hline $\begin{array}{l}\text { 7. When I want to feel happier } \\
\text { about something, I change the way } \\
\text { I'm thinking about it. }\end{array}$ & & & & & \\
\hline $\begin{array}{l}\text { 8. I control my feelings about } \\
\text { things by changing the way I think } \\
\text { about them. }\end{array}$ & & & & & \\
\hline $\begin{array}{l}\text { 9. When I'm feeling bad (e.g., sad, } \\
\text { angry, or worried), I'm careful not } \\
\text { to show it. }\end{array}$ & & & & & \\
\hline $\begin{array}{l}\text { 10. When I want to feel less bad } \\
\text { (e.g., sad, angry, or worried) about } \\
\text { something, I change the way I'm } \\
\text { thinking about it. }\end{array}$ & & & & & \\
\hline
\end{tabular}


Gullone, E., \& Taffe, J. (2012). The Emotion Regulation Questionnaire for Children and Adolescents (ERQ-CA): A psychometric evaluation. Psychological Assessment, 24, $402-$ 417. 
Appendix 5

The Regulatory Emotional Self-Efficacy Scale - Adapted

Adapted for Children from Caprara \& Gerbino, 2001 
The Regulatory Emotional Self-Efficacy Scale - Adapted

Adapted for Children from Caprara \& Gerbino, 2001

\begin{tabular}{|c|c|c|c|c|c|}
\hline & $\begin{array}{c}1 \\
\text { Not } \\
\text { good }\end{array}$ & $\begin{array}{c}2 \\
\text { Not } \\
\text { well }\end{array}$ & $\begin{array}{c}3 \\
\text { Not } \\
\text { good } \\
\text { or bad }\end{array}$ & $\begin{array}{c}4 \\
\text { Good }\end{array}$ & $\begin{array}{c}5 \\
\text { Very } \\
\text { Good }\end{array}$ \\
\hline $\begin{array}{l}\text { How good are you at expressing happiness } \\
\text { when something good happens to you? }\end{array}$ & & & & & \\
\hline $\begin{array}{l}\text { How good are you at feeling happy or proud } \\
\text { when achieve something you wanted to? }\end{array}$ & & & & & \\
\hline $\begin{array}{l}\text { How good are you at managing bad feelings } \\
\text { (for example, angry, nervous, or sad) when } \\
\text { you are scolded by your parents or someone } \\
\text { else who is important to you? }\end{array}$ & & & & & \\
\hline $\begin{array}{l}\text { How good are you at keeping yourself from } \\
\text { feeling sad or discouraged when you feel } \\
\text { lonely? }\end{array}$ & & & & & \\
\hline $\begin{array}{l}\text { How good are you at being happy over } \\
\text { things you are successful at? }\end{array}$ & & & & & \\
\hline $\begin{array}{l}\text { How good are you at getting over being } \\
\text { annoyed quickly for unfair things you have } \\
\text { experienced? }\end{array}$ & & & & & \\
\hline $\begin{array}{l}\text { How good are you at keeping yourself from } \\
\text { feeling discouraged when someone strongly } \\
\text { criticizes you? }\end{array}$ & & & & & \\
\hline $\begin{array}{l}\text { How good are you at reducing how upset } \\
\text { you are feeling when someone doesn't } \\
\text { appreciate you like you deserve? }\end{array}$ & & & & & \\
\hline $\begin{array}{l}\text { How good are you at expressing that you're } \\
\text { having a good time at a party? }\end{array}$ & & & & & \\
\hline $\begin{array}{l}\text { How good are you at avoiding getting upset } \\
\text { (for example, angry, nervous, or sad) when } \\
\text { other people are giving you a hard time? }\end{array}$ & & & & & \\
\hline
\end{tabular}




\begin{tabular}{|l|l|l|l|l|}
\hline $\begin{array}{l}\text { How good are you at avoiding flying off the } \\
\text { handle (getting really mad and making a } \\
\text { scene) when you get angry? }\end{array}$ & & & & \\
\end{tabular}

Caprara, G. V., \& Gerbino, M. (2001). The capacity to regulate negative affect and to express positive affect. In G. V. Caprara (Ed.), Self-efficacy assessment (pp. 35-50). Trento, Italy: Edizioni Erickson. 
Appendix 6

Emotions as a Child Inventory (Child Report)

O’Neal \& Magai, 2005 


\section{Emotions as a Child Inventory}

1. Think of a time when you felt sad. When you felt $s a d$, what would your mother do?

\begin{tabular}{|c|c|c|c|c|c|c|c|}
\hline & $\begin{array}{c}1 \\
\text { Not } \\
\text { Like } \\
\text { My } \\
\text { Mom }\end{array}$ & 2 & 3 & 4 & 5 & 6 & $\begin{array}{c}7 \\
\text { Exactly } \\
\text { Like } \\
\text { My } \\
\text { Mom }\end{array}$ \\
\hline She usually is not around & & & & & & & \\
\hline She tells you not to worry & & & & & & & \\
\hline She gets sad, too. & & & & & & & \\
\hline She asks you about it. & & & & & & & \\
\hline She calls you a crybaby. & & & & & & & \\
\hline She usually doesn't notic & & & & & & & \\
\hline $\begin{array}{l}\text { She jokes with you about } \\
\text { it. }\end{array}$ & & & & & & & \\
\hline She gets all upset. & & & & & & & \\
\hline $\begin{array}{l}\text { She understands why you } \\
\text { are sad. }\end{array}$ & & & & & & & \\
\hline $\begin{array}{l}\text { She shows she does NOT } \\
\text { like your being sad. }\end{array}$ & & & & & & & \\
\hline She ignores you. & & & & & & & \\
\hline She tells you to cheer up. & & & & & & & \\
\hline She comforts you. & & & & & & & \\
\hline $\begin{array}{l}\text { She gives you a disgusted } \\
\text { look. }\end{array}$ & & & & & & & \\
\hline $\begin{array}{l}\text { She buys you something } \\
\text { you like. }\end{array}$ & & & & & & & \\
\hline $\begin{array}{l}\text { She helps you deal with } \\
\text { the issue. }\end{array}$ & & & & & & & \\
\hline
\end{tabular}


2. Think of a time when you felt angry. When you felt angry, what would your mother do?

\begin{tabular}{|c|c|c|c|c|c|c|c|}
\hline & $\begin{array}{c}1 \\
\\
\text { Not } \\
\text { Like } \\
\text { My } \\
\text { Mom }\end{array}$ & 2 & 3 & 4 & 5 & 6 & $\begin{array}{c}7 \\
\text { Exactly } \\
\text { Like } \\
\text { My } \\
\text { Mom }\end{array}$ \\
\hline She usually is not around & & & & & & & \\
\hline $\begin{array}{l}\text { She tells you to change } \\
\text { your attitude. }\end{array}$ & & & & & & & \\
\hline She gets angry with you. & & & & & & & \\
\hline $\begin{array}{l}\text { She finds out what make } \\
\text { you angry. }\end{array}$ & & & & & & & \\
\hline $\begin{array}{l}\text { She tells you that you are } \\
\text { bad. }\end{array}$ & & & & & & & \\
\hline $\begin{array}{l}\text { Most times she doesn't } \\
\text { notice. }\end{array}$ & & & & & & & \\
\hline $\begin{array}{l}\text { She jokes with you about } \\
\text { it. }\end{array}$ & & & & & & & \\
\hline She yells back at you. & & & & & & & \\
\hline $\begin{array}{l}\text { She understands why you } \\
\text { feel angry. }\end{array}$ & & & & & & & \\
\hline She punishes you. & & & & & & & \\
\hline She ignores you. & & & & & & & \\
\hline She tells you to keep qui & & & & & & & \\
\hline She talks it out with you. & & & & & & & \\
\hline $\begin{array}{l}\text { She says you should be } \\
\text { ashamed. }\end{array}$ & & & & & & & \\
\hline $\begin{array}{l}\text { She helps you deal with } \\
\text { the problem. }\end{array}$ & & & & & & & \\
\hline
\end{tabular}


3. Think of a time when you felt afraid. When you felt afraid, what would your mother do?

\begin{tabular}{|c|c|c|c|c|c|c|c|}
\hline & $\begin{array}{c}1 \\
\\
\text { Not } \\
\text { Like } \\
\text { My } \\
\text { Mom }\end{array}$ & 2 & 3 & 4 & 5 & 6 & $\begin{array}{c}7 \\
\text { Exactly } \\
\text { Like } \\
\text { My } \\
\text { Mom }\end{array}$ \\
\hline She usually is not aroun & & & & & & & \\
\hline $\begin{array}{l}\text { She tells you to not to } \\
\text { worry. }\end{array}$ & & & & & & & \\
\hline She gets scared herself. & & & & & & & \\
\hline $\begin{array}{l}\text { She asks you what's } \\
\text { wrong. }\end{array}$ & & & & & & & \\
\hline She tells you to grow up & & & & & & & \\
\hline She doesn't notice. & & & & & & & \\
\hline $\begin{array}{l}\text { She jokes with you abou } \\
\text { it. }\end{array}$ & & & & & & & \\
\hline $\begin{array}{l}\text { She helps you deal with } \\
\text { the situation. }\end{array}$ & & & & & & & \\
\hline She punishes you. & & & & & & & \\
\hline She ignores you. & & & & & & & \\
\hline She distracts you. & & & & & & & \\
\hline She holds you. & & & & & & & \\
\hline She makes fun of you. & & & & & & & \\
\hline $\begin{array}{l}\text { She tells you not to be } \\
\text { frightened. }\end{array}$ & & & & & & & \\
\hline $\begin{array}{l}\text { She helps you deal with } \\
\text { the problem. }\end{array}$ & & & & & & & \\
\hline
\end{tabular}


4. Think of a time when you felt ashamed. When you felt ashamed, what would your mother do?

\begin{tabular}{|c|c|c|c|c|c|c|c|}
\hline & $\begin{array}{c}1 \\
\\
\text { Not } \\
\text { Like } \\
\text { My } \\
\text { Mom }\end{array}$ & 2 & 3 & 4 & 5 & 6 & $\begin{array}{c}7 \\
\text { Exactly } \\
\text { Like } \\
\text { My } \\
\text { Mom }\end{array}$ \\
\hline She usually is not around & & & & & & & \\
\hline $\begin{array}{l}\text { She tells you to not to } \\
\text { worry. }\end{array}$ & & & & & & & \\
\hline $\begin{array}{l}\text { She feels embarrassed of } \\
\text { you. }\end{array}$ & & & & & & & \\
\hline She asks you about it. & & & & & & & \\
\hline $\begin{array}{l}\text { She says you are acting } \\
\text { like a baby. }\end{array}$ & & & & & & & \\
\hline She doesn't notice. & & & & & & & \\
\hline $\begin{array}{l}\text { She jokes with you about } \\
\text { it. }\end{array}$ & & & & & & & \\
\hline She gets upset herself. & & & & & & & \\
\hline She hugs you. & & & & & & & \\
\hline She puts you down for it & & & & & & & \\
\hline She ignores you. & & & & & & & \\
\hline She says not to worry. & & & & & & & \\
\hline She comforts you. & & & & & & & \\
\hline $\begin{array}{l}\text { She tells you you are } \\
\text { foolish. }\end{array}$ & & & & & & & \\
\hline $\begin{array}{l}\text { She says it wasn't worth } \\
\text { getting upset about. }\end{array}$ & & & & & & & \\
\hline $\begin{array}{l}\text { She helps you solve the } \\
\text { problem. }\end{array}$ & & & & & & & \\
\hline
\end{tabular}


O’Neal, C. R., \& Magai, C. (2005). Do parents respond in different ways when children feel different emotions? The emotional context of parenting. Development and Psychopathology, 17, 467-487. 
Appendix 7

Social Media Advertisement 


\section{ATTENTION MOTHERS OF CHILDREN AGES 10-12:}

If you are at least 18 years old and the mother of a child aged 10-12 years old, you are eligible to participate with your child in a West Virginia University IRB-approved research study on influences on parenting and children's emotional development. The purpose of this study was to explore how parents and children influence one another, and how parenting processes affect children's emotional development. If you qualify and choose to participate, you will receive $\$ 25.00$. This study involves you and your child completing questionnaires in one of several ways - online, in paper-and-pencil form at your home or other convenient location (applicable in the Morgantown, WV area), or over the telephone. This will take approximately 30 minutes each for you and your child to complete. If you have questions or would like to preview the surveys, please e-mail the address below.

If you are interested in participating, please contact Katie Rasmussen by e-mail:

Katie.Rasmussen@mail.wvu.edu or go to:

MOM LINK:

https:/wvuhre.az1.qualtrics.com/SE/?SID=SV_42tocrvEyQtq91H

CHILD LINK:

https:/wvuhre.az1.qualtrics.com/SE/?SID=SV_b2VHmYVGVecxlMV

This study is being conducted by Dr. Amy E. Root, Ph.D. and Katie Rasmussen, M.A. in the Department of Learning Sciences and Human Development at West Virginia University

Contact Amy Root AEKennedy@mail.wvu.edu or Katie Rasmussen

Katie.Rasmussen@mail.wvu.edu)with questions.

Note: IRB approval is on file for this study. 
Appendix 8

Study Flyer 

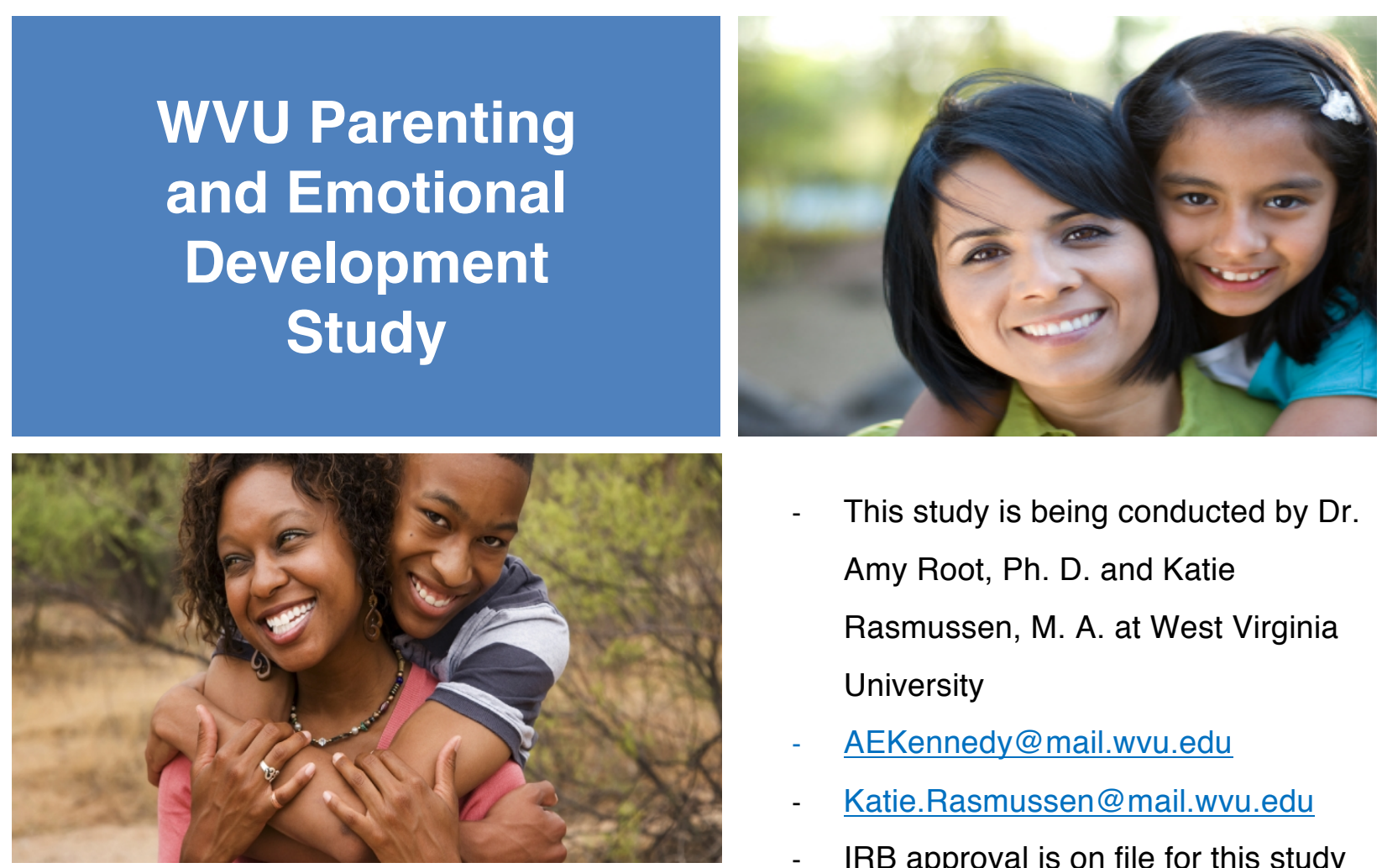

- This study is being conducted by Dr.

Amy Root, Ph. D. and Katie

Rasmussen, M. A. at West Virginia

University

- $\quad$ AEKennedy@mail.wvu.edu

- Katie.Rasmussen@mail.wvu.edu

- $\quad$ IRB approval is on file for this study

If you are at least 18 years old and the mother of a child aged 10-12 years old, you are eligible to participate with your child in a West Virginia University IRB-approved research study on influences on parenting and children's emotional development. The purpose of this study is to explore how parents and children influence one another, and how parenting processes affect children's emotional development. If you qualify and choose to participate, you will receive $\$ 25.00$. This study involves you and your child completing questionnaires in one of several ways - online, in paper-and-pencil form at your home or other convenient location (applicable in the Morgantown, WV area), or over the telephone. This will take approximately 30 minutes each for you and your child to complete. If you have questions or would like to preview the surveys, please e-mail the address below.

If you are interested in participating, please contact Katie Rasmussen by e-mail: Katie.Rasmussen@mail.wvu.edu or go to:

\section{MOTHER LINK:}

https://wvuhre.az1.qualtrics.com/SE/?SID=SV_42tocrvEyQtq9IH

CHILD LINK:

https://wvuhre.az1.qualtrics.com/SE/?SID=SV_b2VHmYVGVecxIMV

Thanks for your consideration! 
Appendix 9

Electronic Cover Letter 


\section{Dear Participant,}

This letter is a request for you and your child to take part in a research project to explore how children and parents influence parents' responses to children's emotions, and how these responses affect children's emotional development. This project is being conducted by Katie Rasmussen, M.A. in College of Education and Human Services at West Virginia University under the supervision of Dr. Amy Root, Associate Professor in the Department of Learning Sciences and Human Development.

We are asking mothers and their children aged $10-12$ years to complete separate batteries of 5 surveys each. Should you choose to participate, the questionnaires will take each person approximately 30 minutes to complete and each mother-child pair will receive $\$ 25.00$.

Mothers must be over 18 years of age to participate, and your involvement in this project will be kept as confidential as legally possible. All data will be reported in the aggregate, and no names will be used in research reports that may originate from this project. Your participation is completely voluntary, and there is no penalty for not participating. You or your child may skip any question you do not wish to answer and you may discontinue at any time.

We hope that you will participate in this research project, as it could be beneficial in understanding how both parents and children themselves contribute to the parent-child relationship and children's emotional development. Please note West Virginia University's Institutional Review Board acknowledgement of this project is on file.

Thank you very much for your time! If you would like to participate or should you have any questions about this letter, the study, or would like to preview the surveys before choosing to participate, please contact Katie Rasmussen by e-mail (Katie.Rasmussen@mail.wvu.edu) or Amy Root at (304) 293-0380 or by e-mail (Amy.Kennedy@mail.wvu.edu).

Please note this survey works best on desktop or laptop computers rather than mobile phones. Thank you for your time and help with this project!

Best wishes,

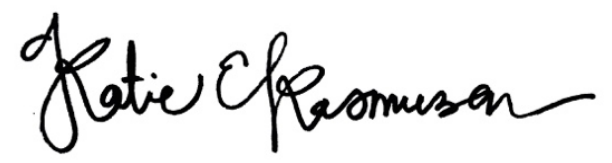

Katie E. Rasmussen, M. A., Doctoral Candidate

Department of Learning Sciences and Human Development

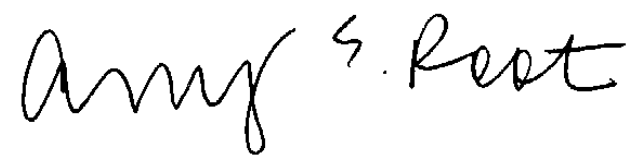

Amy E. Root, Ph.D.

Department of Learning Sciences and Human Development 